\title{
The Next Generation Photoinjector
}

\author{
by Dennis T. Palmer
}

Ph.D. Thesis 


\title{
THE NEXT GENERATION PHOTOINJECTOR
}

\author{
A DISSERTATION \\ SUBMITTED TO THE DEPARTMENT OF APPLIED PHYSICS \\ AND THE COMMITTEE ON GRADUATE STUDIES \\ OF STANFORD UNIVERSITY \\ IN PARTIAL FULFILLMENT OF THE REQUIREMENTS \\ FOR THE DEGREE OF \\ DOCTOR OF PHILOSOPHY
}

Dennis Thomas Palmer

June 1998 
(C) Copyright by Dennis Thomas Palmer 1998

All Rights Reserved 
I certify that I have read this dissertation and that in my opinion it is fully adequate, in scope and quality, as a dissertation for the degree of Doctor of Philosophy.

\section{Professor Roger H. Miller (Principal Adviser)}

I certify that I have read this dissertation and that in my opinion it is fully adequate, in scope and quality, as a dissertation for the degree of Doctor of Philosophy.

\section{Professor Robert H. Siemann}

I certify that I have read this dissertation and that in my opinion it is fully adequate, in scope and quality, as a dissertation for the degree of Doctor of Philosophy.

Professor Helmut Wiedemann

Approved for the University Committee on Graduate Studies: 


\section{ABSTRACT}

This dissertation will elucidate the design, construction, theory, and operation of the Next Generation Photoinjector (NGP). This photoinjector is comprised of the BNL/SLAC/UCLA 1.6 cell symmetrized S-band photocathode radio frequency (rf) electron gun and a single emittance-compensation solenoidal magnet. This photoinjector is a prototype for the Linear Coherent Light Source X-ray Free Electron Laser operating in the $1.5 \AA$ range. Simulations indicate that this photoinjector is capable of producing a $1 \mathrm{nC}$ electron bunch with transverse normalized emittance less than 1 $\pi \mathrm{mm}$ mrad were the cathode is illuminated with a 10 psec longitudinal flat top pulse. Using a Gaussian longitudinal laser profile with a full width half maximum (FWHM) of 10 psec, simulation indicates that the NGP is capable of producing a normalized rms emittance of $2.50 \pi \mathrm{mm} \operatorname{mrad}$ at $1 \mathrm{nC}$.

Using the removable cathode plate we have studied the quantum efficiency (QE) of both copper and magnesium photo-cathodes. The $\mathrm{Cu}$ QE was found to be $4.5 \times 10^{-5}$ with a $25 \%$ variation in the QE across the emitting surface of the cathode, while supporting a field gradient of $125 \frac{\mathrm{MV}}{\mathrm{m}}$.

At low charge, the transverse normalized rms emittance, $\epsilon_{\mathrm{n}, \mathrm{rms}}$, produced by the $\mathrm{NGP}$ is $\epsilon_{\mathrm{n}, \mathrm{rms}}=1.2 \pi \mathrm{mm} \operatorname{mrad}$ for $\mathrm{Q}_{\mathrm{T}}=0.3 \mathrm{nC}$. The $95 \%$ electron beam bunch length was measured to 10.9 psec.

The emittance due to the finite magnetic field at the cathode has been studied. The scaling of this magnetic emittance term as a function of cathode magnetic field was found to be $0.01 \pi \mathrm{mm}$ mrad per Gauss.

The 1.6 cell rf gun has been designed to reduce the dipole field asymmetry of the longitudinal accelerating field. Low level rf measurements show that this has in fact 
been accomplished, with an order of magnitude decrease in the dipole field. High power beam studies also show that the dipole field has been decreased.

An upper limit of the intrinsic non-reducible thermal emittance of a photocathode under high field gradient was found to be $\epsilon_{\mathrm{n}, \mathrm{rms}}=0.8 \pi \mathrm{mm} \mathrm{mrad}$. Agreement is found between our theoretical calculation of the thermal emittance, $\epsilon_{\mathrm{o}}=0.62 \pi \mathrm{mm}$ mrad, and our experimental results, after taking into account all of the emittance contributions terms.

The $1 \mathrm{nC}$ emittance was found to be $\epsilon_{\mathrm{n}, \mathrm{rms}}=4.75 \pi \mathrm{mm} \mathrm{mrad}$ with a $95 \%$ electron beam bunch length of 14.7 psec. Systematic bunch length measurements showed electron beam bunch lengthening due the electron beam charge.

We will show that the discrepancy between measurement and simulation is due to three effects. The major effect is due to the variation of the QE in the photo-emitting area of the $\mathrm{Cu}$ cathode. Also, space charge emittance blowup in the transport line will be shown to be a significant effect because the electron beam is still in the space charge dominated regime. The last effect, which has been observed experimentally, is the electron bunch lengthening as a function of total electron bunch charge. 


\section{Acknowledgments}

It is difficult to remember all the people that have had an influence on me throughout my life. Please do not feel slighted if I forget to mention you. Over my graduate school years at Stanford University I have interacted with many different people and research groups all over the world. My dissertation project, The Next Generation Photoinjector, was a multi-institutional collaboration that involved BNL, UCLA, and SLAC/SSRL. I want to thank the leaders of this collaboration: Ilan Ben-Zvi, Roger H. Miller, Claudio Pellegrini and Herman Winick, for allowing me the opportunity to work at the forefront of accelerator research.

The technical staff at the ATF: Bill Cahill, Mark Montemagno, and Bob Harrington; deserve a big hand for all the help they provided me during the cold testing, installation, and beam dynamics studies. The UCLA machine shop staff that consists of: Al Casillas, Ken Luk, and Harry Lockart; thanks guys. Without their attention to detail the 1.6 cell rf gun would have never reached the field levels that it did. The staff at the SLAC's light fabrication shop conducted the gun's final machining, cleaning, and brazing also deserve a big hand. Ali Farvid, Dale Sartain, Howard Baruz, Eric Lundahl, Ossie Millican, Zoltan Bordas, thanks guys for all your hard work. At SSRL, Jim Weaver and Mike Nalls, were instrumental in getting high power rf delivered to the 1.6 cell rf gun for breakdown studies.

I had a great time in graduate school, especially my first year, where Dave Wehner and I spent hours working together doing Quantum Mechanics homework sets together. Dave went on to be an investment banker. He might have made the right choice. My friend and colleague Robert Holtzapple listened to my tirades on everything from politics to race over lunch every Friday at Su Hong for a couple years until 
he graduated and went off to Cornell. He should get a medal for the understanding he showed towards me. Boris, Dave, and Marc, whom all move up one more notch in the chain of command, it is well worth it. I want to thank Mark Hogan for being a real friend to me when I needed his help. Mark, thanks for bailing me out of that jam. We will have a great time doing our post-doc in ARDB.

Jamie Rosenzweig, Luca Serafini, and XiJie Wang are close friends and colleagues of mine. I have become a better physicist and more importantly a better person because of them. I want to thank Robert Siemann for hiring me right out of ARDA, in violation of his incest rule. Thanks Bob. I also would like to thank my thesis advisor, Roger H. Miller, for standing beside me when the need arose. I will give one piece of advice to all prospective graduate students. Get an advisor that is a real human being. I did and it made graduate school a great experience.

I want to thank my English editor, Lisa Palmer, for reading through my dissertation. Lisa, I hope that it does tell a story.

I have had an unconventional path to a Ph.D. graduating high school in Del City Oklahoma and going straight into the United States Navy. In the Navy, I met a very diverse group of people whom I still call friends.

The Hutchinson family of Bulter PA has a son, Richard, that also joined the Navy. Richard and I have been friends since 1977. It was not unusual for me to wake up in the clown room in the Hutchinson family home and hear Mrs. Hutchinson say to her husband Jim Hutchinson, "Its only Dennis, he must have dropped in last night". But you should know that when I drop in it was from the Middle East, the Caribbean, or some God forsaken part of the world where I had just spent six months on board the USS Mahan DDG-42. The Hutchinson family always made me feel at home. Richard and his brother Dan have been like brothers to me and I could not have made it this far without their love and support. Hey Bra, we have made it a long way from that barracks room in Great Lakes.

Of course I have been influenced by the ladies that have come into my life. Cathy, Inge, Kim, Laura, Lisa, and Lindamaris, I will always treasure what we had together. The lady that gets me to settle down will get a man that is the sum of all your contributions. 
My brother Bobby Palmer was the first to get his Ph.D. and his example was an inspiration to me. Bobby don't forget that you owe me $\$ 100$ for the $\mathrm{LT}_{\mathrm{E} X} \mathrm{X}$ bet. My sister-in-law, Masami, took care of my mother while she was dying, this is a debt I can never repay, but I will always try. My nieces, Ashley and Alyssa, always bring a smile to my face and provide me with unconditional love that I will always crave. My mother, Mary Wilson, was always there for me and I know she will always be with me in spirit. I love you Mom. 


\section{Contents}

ABSTRACT iv

Acknowledgments $\quad$ vi

1 Introduction 1

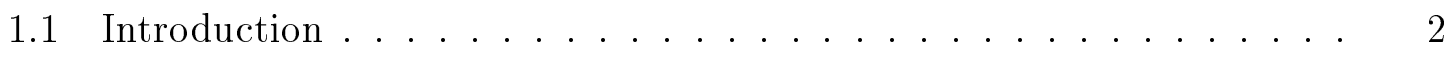

1.2 Emittance Compensation Modeling . . . . . . . . . . . 3

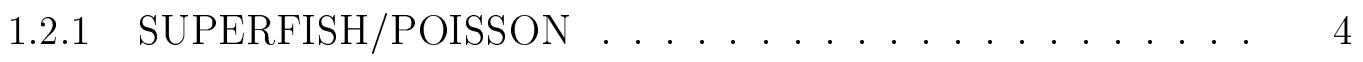

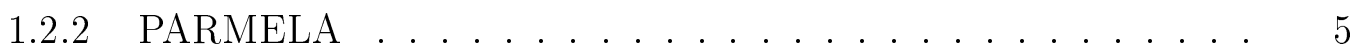

1.3 Single Pass FEL Theory $\ldots \ldots \ldots \ldots \ldots \ldots$

1.3.1 Emittance Requirement $\ldots \ldots \ldots \ldots \ldots$

1.3.2 FEL Parameter Optimization $\ldots \ldots \ldots \ldots$

1.4 Electron Sources $\ldots \ldots \ldots \ldots \ldots$

1.4.1 DC Electron Sources . . . . . . . . . . . . . . 11

1.4 .2 rf Electron Sources . . . . . . . . . . . . . . . 12

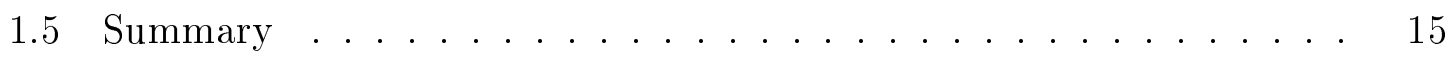

2 Beam Dynamics: Theory and Simulations 16

2.1 Introduction . . . . . . . . . . . . . . . . . 17

2.2 Transverse Linear Space Charge

Emittance Compensation . . . . . . . . . . . . . . 17

2.3 Beam Dynamics Theory . . . . . . . . . . . . . . . 21

2.3.1 Liouville's Theorem . . . . . . . . . . . . . . . . 21

2.3.2 Emittance Concepts . . . . . . . . . . . . . . . 23 
2.3 .3 Emittance Terms . . . . . . . . . . . . . . . . . . . 24

2.3.4 Emittance Estimation in the Zero Charge Limit . . . . . . . 35

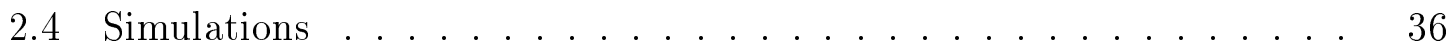

2.4.1 Electron Beam Energy, $\gamma m_{o} c^{2}$, Versus Laser Injection Phase, $\theta_{0}$, and Accelerating Field Gradient, $\mathrm{E}_{\mathrm{o}} \ldots \ldots \ldots 38$

$2.4 .2 \epsilon_{\mathrm{n}, \mathrm{rms}}$ Versus $\mathrm{E}_{\mathrm{o}}$ and $\mathrm{B}_{\mathrm{z}} \ldots \ldots \ldots \ldots \ldots \ldots$

$2.4 .3 \quad \epsilon_{\mathrm{n}, \mathrm{rms}}$ Versus $\frac{\mathrm{E}_{2}}{\mathrm{E}_{1}}$ and $\mathrm{B}_{\mathrm{z}} \ldots \ldots \ldots \ldots \ldots 41$

$2.4 .4 \epsilon_{\mathrm{n}, \mathrm{rms}}$ Versus $\mathrm{B}_{\mathrm{z}} \ldots \ldots \ldots \ldots \ldots \ldots \ldots \ldots$

$2.4 .5 \epsilon_{\mathrm{n}, \mathrm{rms}}$ Versus $\theta_{0} \ldots \ldots \ldots \ldots \ldots \ldots \ldots \ldots$

$2.4 .6 \epsilon_{\mathrm{n}, \mathrm{rms}}$ Versus Total Charge, $\mathrm{Q}_{\mathrm{t}} \ldots \ldots \ldots \ldots \ldots$

$2.4 .7 \epsilon_{\mathrm{n}, \mathrm{rms}}$ Versus Pulse Shape . . . . . . . . . . . . . . . . 49

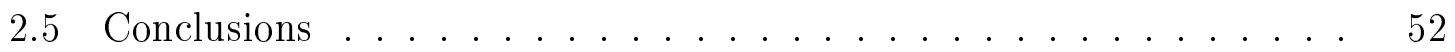

3 Microwave Theory and Measurements $\quad 54$

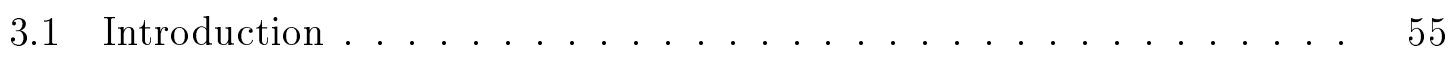

3.2 Microwave Measurement Theory . . . . . . . . . . . . . 55

3.2 .1 Scattering Parameters . . . . . . . . . . . 55

3.2 .2 rf Coupling Constant, $\beta_{\mathrm{rf}} \ldots \ldots \ldots \ldots \ldots \ldots$

3.2 .3 Bead Perturbation . . . . . . . . . . . . 60

$3.3 \quad 1.6$ Cell rf Gun Design . . . . . . . . . . . . . . . . . 61

3.3.1 General rf and Beam Dynamics Design Issues . . . . . . . 61

3.3.2 Manufacturing Techniques and Tolerances . . . . . . . . 65

3.3 .3 Cleaning Issues . . . . . . . . . . . . . . . . . . 68

3.4 Electro-magnetic Simulations _ . . . . . . . . . . . 70

3.4.1 Field Balance Versus Mode Separation . . . . . . . . . . 72

3.5 Frequency Measurement . . . . . . . . . . . . . 76

3.5.1 Independent Cell Frequencies . . . . . . . . . . . 77

3.6 Longitudinal Field Measurements . . . . . . . . . . . . . 78

3.7 Transverse Field Measurements _ . . . . . . . . . . . . . . 79

3.7.1 Transverse Disk Pulls . . . . . . . . . . . . . . . . . 81

3.7 .2 Needle Rotation Measurements . . . . . . . . . . . . . 83 
3.8 Waveguide to $\pi$-mode Coupling . . . . . . . . . . . . 88

3.8.1 Bethe Hole Coupling Theory . . . . . . . . . . . . . . . 88

$3.8 .2 \quad$ Experimental Data . . . . . . . . . . . . . . . . 89

3.9 Conclusions . . . . . . . . . . . . . . . . . . . . . . 94

4 Solenoidal Magnet $\quad 96$

4.1 Introduction . . . . . . . . . . . . . . . . . . . 97

4.2 POISSON and PARMELA

Simulation Program . . . . . . . . . . . . . . . 98

4.2.1 Production Magnet Design . . . . . . . . . . . . . . 100

4.3 Solenoidal Magnet Design _. . . . . . . . . . . . . . . 102

4.3 .1 Field Measurements . . . . . . . . . . . . . . . 103

4.4 Bucking Magnet Design _ . . . . . . . . . . . . . 106

4.4 .1 Field Measurements _. . . . . . . . . . . . . . 106

$4.5 \cos (\theta)$ deflection magnet $\ldots \ldots \ldots \ldots \ldots \ldots$

$4.5 .1 \quad$ Field Measurements _... . . . . . . . . . . . . . 109

5 Experimental Results $\quad 111$

5.1 Introduction . . . . . . . . . . . . . . . . . . . . . . . . 112

5.2 Facility Overview . . . . . . . . . . . . . . . . . . . . . 112

$5.2 .1 \quad$ Linac System . . . . . . . . . . . . . . . . . . 113

$5.2 .2 \quad$ Laser System . . . . . . . . . . . . . . . . . . . 114

5.2 .3 Control System . . . . . . . . . . . . . . . . . 115

5.3 Two Screen Emittance Measurement . . . . . . . . . . . . . 118

5.3.1 Theory of Emittance Measurement _. . . . . . . . . . 118

5.3.2 Experimental Measurement Technique . . . . . . . . . 120

5.3 .3 Systematic Errors . . . . . . . . . . . . . . . . . . . 122

5.4 Quantum Efficiency Studies . . . . . . . . . . . . . . . 122

5.4.1 Cathode Preparation Technique . . . . . . . . . . . . 123

5.4 .2 Cathode Studies . . . . . . . . . . . . . . 125

$5.5 \quad$ Low Energy Beam Studies . . . . . . . . . . . . . . . . 131 
5.5.1 Electron Beam Energy, $\gamma \mathrm{m}_{\mathrm{o}} \mathrm{c}^{2}$, Versus Accelerating Field Gradients, $\mathrm{E}_{\mathrm{o}}$, and Laser Injection Phase, $\theta_{0} \ldots \ldots . . . . .131$

5.5.2 Field Enhancement Factor $\beta$. . . . . . . . . . . . 132

5.6 Transverse Phase Space Emittance Studies . . . . . . . . . . . . 133

$5.6 .1 \epsilon_{\mathrm{n}, \mathrm{rms}}$ Versus $\mathrm{B}_{\mathrm{z}} \ldots \ldots \ldots \ldots \ldots . \ldots \ldots$

$5.6 .2 \epsilon_{\mathrm{n}, \mathrm{rms}}$ Versus $\mathrm{Q}_{\mathrm{t}} \ldots \ldots \ldots \ldots . \ldots \ldots$

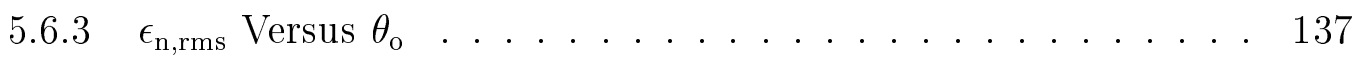

5.7 Longitudinal Phase Space . . . . . . . . . . . . . 137

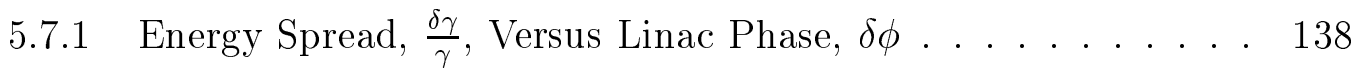

5.7.2 Electron Bunch Length, $\tau_{95 \%}$, Versus Laser Injection Phase, $\theta_{0} 138$

5.8 Multi-pole Field Asymmetry . . . . . . . . . . . . . . . . . . . 140

5.8.1 Experimental Setup . . . . . . . . . . . . . 140

5.8.2 Experimental Beam Dynamics Studies . . . . . . . . . . 142

5.9 Cathode Magnetic Field Asymmetry . . . . . . . . . . . . . . . 144

5.9.1 Experimental Setup . . . . . . . . . . . . 145

5.9 .2 Experimental Beam Dynamics Studies . . . . . . . . . 146

$5.101 \mathrm{nC}$ Emittance Studies . . . . . . . . . . . . . . 149

5.11 Summary . . . . . . . . . . . . . . . . 151

5.11 .1 Quantum Efficiency Studies ............ 151

5.11 .2 Energy Studies . . . . . . . . . . . . . . . 151

5.11 .3 Transverse Normalized rms Emittance Studies . . . . . . . . . 152

5.11 .4 Longitudinal Phase Space . . . . . . . . . . . . . 153

5.11 .5 Multi-Pole Field Studies . . . . . . . . . . . . . . 153

5.11 .6 Cathode Magnetic Field Studies . . . . . . . . . . . . . 154

5.11.7 $1 \mathrm{nC}$ Transverse Normalized rms Emittance Studies . . . . . . 154

6 Conclusions and Future Work $\quad 155$

6.1 Conclusions ........................ 156

6.2 Future Work . . . . . . . . . . . . . . 156

6.2.1 Beam Dynamics Simulations . . . . . . . . . . 157

6.2.2 Magnet and rf Design . . . . . . . . . . . . 157 
6.2.3 Experimental Issues . . . . . . . . . . . . . . . . 158

6.2.4 Advanced Beam Diagnostics ............... 161

A Index of Symbols $\quad 162$ 


\section{List of Tables}

1.1 LCLS photoinjector parameters. . . . . . . . . . . . 7

1.2 Universal scaling law fit parameters. . . . . . . . . . . . . . 10

1.3 Electron beam parameters for the Boeing thermionic DC gun/injector

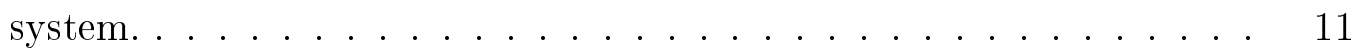

1.4 SLAC polarized DC gun/injector system. . . . . . . . . . . . 12

1.5 SSRL thermionic rf gun/injector system. . . . . . . . . . . . . 14

1.6 BNL/ATF Photocathode $\mathrm{rf}$ gun/injector system. . . . . . . . . 15

2.1 Cathode spot size and rf parameters. Where the $\theta_{o}$ is defined from the

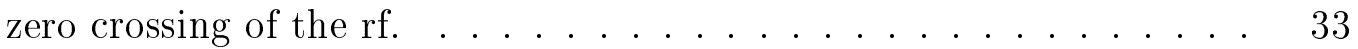

2.2 Estimation of the individual emittance terms in the zero charge limit, in units of $\pi$ mm mrad. . . . . . . . . . . . . . . . . . 35

2.3 Definition of symbols used throughout Section 2.4. . . . . . . . . 37

2.4 PARMELA simulation parameters for $\gamma$ versus $\theta_{0}$ and $\mathrm{E}_{\mathrm{o}}$, using a 10 psec $\mathrm{FWHM}_{\mathrm{z}}$ flat-top longitudinal laser profile. Note that the solenoidal field is constant for all values of $\mathrm{E}_{\mathrm{o}}$ and $\theta_{\mathrm{o}} \ldots \ldots \ldots 38$

2.5 Comparison of Serafini's analytical results to PARMELA simulations of the maximum energy out of the NGP as a function of $\mathrm{E}_{0} . \ldots 40$

2.6 PARMELA simulation parameters for $\epsilon_{\mathrm{n}, \mathrm{rms}}$ versus $\mathrm{E}_{\mathrm{o}}$ and $\mathrm{B}_{\mathrm{z}}$. . . . 40

2.7 PARMELA simulation parameters for $\epsilon_{\mathrm{n}, \mathrm{rms}}$ versus $\frac{\mathrm{E}_{2}}{\mathrm{E}_{1}} \ldots \ldots \ldots 42$

2.8 PARMELA simulation parameters for $\epsilon_{\mathrm{n}, \mathrm{rms}}$ versus $\mathrm{B}_{\mathrm{z}} \ldots \ldots \ldots 44$

2.9 PARMELA simulation parameters for $\epsilon_{\mathrm{n}, \mathrm{rms}}$ versus $\theta_{0} \ldots \ldots \ldots$

2.10 PARMELA simulation parameters for $\epsilon_{\mathrm{n}, \mathrm{rms}}$ versus Total Charge, $\mathrm{Q}_{\mathrm{t}}$. 48

2.11 PARMELA simulation parameters for $\epsilon_{\mathrm{n}, \mathrm{rms}}$ versus Pulse Shape. . . . 49 
2.12 2-D PARMELA results for both Gaussian and flat-top longitudinal laser profiles, $\epsilon_{\mathrm{n}, \mathrm{rms}}$ versus $\mathrm{B}_{\mathrm{z}}$ and $\theta_{\mathrm{o}}$ and $\epsilon_{\mathrm{n}, \mathrm{rms}}$ versus $\mathrm{B}_{\mathrm{z}}$ and $\mathrm{R}_{\mathrm{o}} \ldots .52$

3.1 1.6 cell rf gun mechanical dimensions and tolerances for Fig. 3.6. . . . 67

3.2 SUPERFISH simulations results of the 1.6 cell $\mathrm{rf}$ gun, for $\pi$-mode balanced fields. . . . . . . . . . . . . . . . . 71

3.3 Detuning effect due to cavity dimensional changes, in units of $\frac{\mathrm{MHz}}{\mathrm{mill}}$. The cavity dimensions correspond to Fig. 3.6. . . . . . . . . . . . 72

3.4 Dipole offset, $\mathrm{C}$, before and after symmetrization for both resistive and capacitive coupling. . . . . . . . . . . . . . 82

3.5 Multi-pole coefficients of the Fourier series before and after symmetrization for both resistive and capacitive coupling. . . . . . . . . . . 87

3.6 Summary of shorting experiments. . . . . . . . . . . . . . . 91

3.7 Parameters for position of the inductive post. . . . . . . . . . . . 92

3.8 Low power rf experimental results, before and after inductive post matching. . . . . . . . . . . . . . . . 92

4.1 Emittance compensation solenoidal magnet mechanical and electric parameters. . . . . . . . . . . . . . . 103

5.1 Estimated value of the systematic errors . . . . . . . . . . . 122

5.2 Beam study parameters. . . . . . . . . . . . . 133

5.3 Comparison of inferred experimental results, PARMELA simulation, and theory for the emittance growth due to the cathode magnetic field. 148

$5.41 \mathrm{nC}$ emittance results. . . . . . . . . . . . . . . . 149

5.5 High charge emittance study results before and after LAEEE. . . . . 151 


\section{List of Figures}

1.1 The Next Generation Photoinjector. . . . . . . . . . . . . 2

1.2 Artist's view of the proposed Linear Coherent Light Source (LCLS) at the Stanford Linear Accelerator Center. . . . . . . . . . . . . . . 3

1.32 -D view of the overlap of the electron and FEL beams. . . . . . . 8

1.4 Boeing Corporation thermionic DC injector. . . . . . . . . . . . 11

1.5 SLAC polarized photocathode DC gun. . . . . . . . . . . . . . 12

1.6 SSRL thermionic $\operatorname{rf}$ gun. . . . . . . . . . . . . . . 13

1.7 BNL 1.5 cell side coupled zero mode suppressed photocathode rf gun. 14

2.1 Graphically representation of Carlsten's emittance compensation process. 18

2.2 Phase space density as a function of time. . . . . . . . . . . 21

2.3 Thermal emittance contribution as a function of applied accelerating field gradient. . . . . . . . . . . . . . . . . . 33

2.4 The energy spectrum of photoemitted electrons for $0^{\circ} \mathrm{K}$ and $300^{\circ} \mathrm{K}$. $\quad 34$

2.5 Low energy beam line used in the PARMELA simulations of this section. 36

2.6 Transverse and longitudinal laser profile used in the following PARMELA simulations. . . . . . . . . . . . . . . 37

2.7 PARMELA simulations of the electron beam energy versus laser injection phase and field gradients. . . . . . . . . . . . . . 39

2.8 PARMELA simulation results for $\epsilon_{\mathrm{n}, \mathrm{rms}}$ versus $\mathrm{E}_{\mathrm{o}}$ and $\mathrm{B}_{\mathrm{z}} \ldots \ldots \ldots 41$

2.9 SUPERFISH field maps used in PARMELA to investigate the dependence of $\epsilon_{\mathrm{n}, \mathrm{rms}}$ on field balance. . . . . . . . . . . . . . 43

2.10 PARMELA simulations of $\epsilon_{\mathrm{n}, \mathrm{rms}}$ versus $\frac{\mathrm{E}_{2}}{\mathrm{E}_{1}} \ldots \ldots \ldots . \ldots . \ldots 43$ 
2.11 PARMELA simulation results for emittance versus peak magnet field. Note that cusp is a artifact of the separates fits used in region A and B. 45

2.12 Diagram of the emittance compensation process. . . . . . . . . . 45

2.13 PARMELA simulations of $\epsilon_{\mathrm{n}, \mathrm{rms}}$ and $\gamma$ versus laser injection phase $\theta_{\mathrm{o}}$ for a longitudinal flat-top laser profile. . . . . . . . . . . . . 47

2.14 PARMELA simulations of $\epsilon_{\mathrm{n}, \mathrm{rms}}$ versus $\mathrm{Q}_{\mathrm{t}}$ at $\mathrm{z}=165 \mathrm{~cm}$ from the

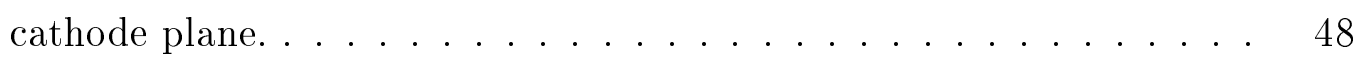

2.15 2-D parameter scans using PARMELA for a 10 psec $\mathrm{FWHM}_{\mathrm{z}}$ flattop longitudinal laser pulse, $\epsilon_{\mathrm{n}, \mathrm{rms}}$ versus cathode spot size, $\mathrm{R}_{\mathrm{o}}$, laser injection phase, $\theta_{0}$, and magnetic field, $\mathrm{B}_{\mathrm{z}}$. . . . . . . . . 50

$2.162-\mathrm{D}$ parameter scans using PARMELA for a $\sigma_{\mathrm{z}}=2.5$ psec Gaussian longitudinal laser pulse, $\epsilon_{\mathrm{n}, \mathrm{rms}}$ versus cathode spot size, $\mathrm{R}_{\mathrm{o}}$, laser injection phase, $\theta_{0}$, and magnetic field, $\mathrm{B}_{\mathrm{z}} \ldots \ldots \ldots . \ldots 51$

3.11 .6 cell rf gun represented as a two port device. . . . . . . . . . 56

3.2 Smith Chart representation of a over-coupled system. . . . . . . . 59

3.31 .6 cell rf gun cross sectional view. . . . . . . . . . . . 62

3.4 0-mode suppression in a magnetically side coupled BNL type rf gun. . 63

3.5 Cavity penetration into the 1.6 cell $\mathrm{rf}$ gun, as seen from the downstream end of the $\operatorname{rfgun}$. . . . . . . . . . . . . . . 64

3.6 Dimension labels corresponding to Table 3.1. . . . . . . . . . 66

$3.7 \mathrm{rf}$ and vacuum iris dimensions after EDM. . . . . . . . . . . . . 68

3.8 Schematic diagram of UHV bake-out stand. . . . . . . . . . . . 69

$3.9 \pi$-mode and 0-mode $\mathrm{E}_{\mathrm{z}}$ field profiles for a balance $\pi$-mode at $2856 \mathrm{MHz} .71$

3.10 Bead pull experimental setup. . . . . . . . . . . . . 72

3.11 Self excited loop used to increase the frequency resolution of our field measurements. . . . . . . . . . . . . . 73

3.12 Field balance versus mode separation. . . . . . . . . . . . 74

3.13 Equivalent circuit LC model for the cell-to-cell coupling. . . . . . . . 74

3.14 Model of modal and independent cell frequency. . . . . . . . . . . 78

3.15 Network analyzer data for balanced fields. . . . . . . . . . . 78 
3.16 Bead pull experimental setup. . . . . . . . . . . . . . 79

3.17 Longitudinal accelerating field measurement with the rf gun out of field balance. ....................... 80

3.18 Resistive and capacitive rf coupling slots designs. . . . . . . . . . 80

3.19 Experimental setups for full cell disk pull. . . . . . . . . . . . 81

3.20 Longitudinal accelerating field as a function of transverse position for the resistive coupling case before and after symmetrization. . . . . . . 82

3.21 Longitudinal accelerating field as a function of transverse position for the capacitive coupling case before and after symmetrization. . . . .

3.22 Transverse and longitudinal views of the needle rotation experimental

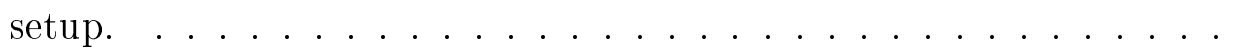

3.23 Longitudinal accelerating field as a function of $\Theta$ for the resistive coupling case before and after symmetrization. . . . . . . . . .

3.24 Longitudinal accelerating field as a function of $\Theta$ for the capacitive coupling case before and after symmetrization. . . . . . . . .

3.253 -D plot of the longitudinal accelerating field for the resistive coupling case, before symmetrization. . . . . . . . . . . . .

3.263 -D plot of the longitudinal accelerating field for the capacitive coupling case, before symmetrization. . . . . . . . . . . .

3.27 Fourier coefficient for the resistive coupling case before and after symmetrization. ..................... 86

3.28 Fourier coefficient for the capacitive coupling case before and after symmetrization. ......................... 87

3.29 Under-coupled impedance locus. . . . . . . . . . . . . . . 90

3.30 Data sheet used to match the waveguide to $\pi$-mode, for an inductive

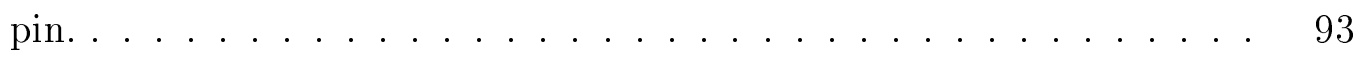

3.31 Impedance locus after inductive post matching. . . . . . . . . . 94

4.1 Drawing of original BNL/ATF air core emittance compensation magnet along with the single emittance compensation magnet. Both magnet designs are shown with their associated rf guns. . . . . . . . 
4.2 Magnet designs investigated using PARMELA. . . . . . . . . . . . 99

4.3 Longitudinal magnetic field profile of the magnet designs in Fig. 4.2. . 99

4.4 PARMELA simulation results of $\epsilon_{\mathrm{n}, \mathrm{rms}}$ versus ECOMP1 magnet position.100

4.5 Final POISSON model of the single emittance compensation magnet. 101

4.6 Solenoidal magnet mechanical drawing. . . . . . . . . . . . . . 102

4.7 Magnetic field measurements for five different excitation currents. . . 104

4.8 Peak magnetic field versus excitation current. . . . . . . . . . . . 104

4.9 Magnetic field measurements. . . . . . . . . . . . . . 105

4.10 Difference in the off axis $(5 \mathrm{~mm})$ and on axis magnetic field measurement at 150 Amperes excitation current. . . . . . . . . . . . 106

4.11 Cathode magnetic field versus peak magnetic field. . . . . . . . . 107

4.12 Effective magnetic length as a function of excitation current. . . . . 107

4.13 Bucking magnet field measurements for 5 and 10 Amperes. . . . . . . 108

4.14 Transverse magnetic field profile. . . . . . . . . . . . . 109

4.15 Integration path inside the $\cos (\theta)$ dipole magnet. . . . . . . . . . 110

5.1 The Next Generation Photoinjector beam line layout. . . . . . . . . 113

5.2 ATF beam-line ........................ 114

5.3 Linac,laser, and rf system block diagram. . . . . . . . . . . . . 115

5.4 Laser system front end. . . . . . . . . . . . . . . . 116

5.5 uv gun hutch layout. . . . . . . . . . . . . . . 117

5.6 Graphical illustration of the two screen emittance measurement method.119

5.7 Total charge versus laser energy before and after laser cleaning. The different data sets are described in the text. . . . . . . . . . . . 124

5.8 Schottky phase scan. . . . . . . . . . . . 126

5.9 Total charge versus polarizer angle for the $\mathrm{Cu}$ cathode. . . . . . . . 127

5.10 Total charge versus polarizer angle for Mg cathode. . . . . . . . . . 127

5.11 Total charge versus laser energy for the copper cathode used in the low charge emittance studies. . . . . . . . . . . . . . . . 128

5.12 Total charge versus laser energy for the Mg cathode. . . . . . . . . 129 
5.13 Normalized charge emission of a copper $(\mathrm{Cu})$ cathode of $1 \mathrm{~mm}$ radius with no laser cleaning. . . . . . . . . . . . . . . 130

5.14 Normalized charge emission of a magnesium $(\mathrm{Mg})$ cathode of $1 \mathrm{~mm}$ radius before and after LAEEE cleaning. . . . . . . . . . . . 130

5.15 Electron beam energy versus laser injection phase for four different cathode fields. . . . . . . . . . . . . . . . 131

5.16 Copper cathode normalized Fowler-Nordheim plot. . . . . . . . . 133

5.17 Experimental beam dynamics results of $\epsilon_{\mathrm{n}, \mathrm{rms}}$ and $\mathrm{R}_{\mathrm{rms}}$ versus $\mathrm{B}_{\mathrm{z}}$. . . 134

5.18 PARMELA simulations of $\epsilon_{\mathrm{n}, \mathrm{rms}}$ and $\mathrm{R}_{\mathrm{rms}}$ versus $\mathrm{B}_{\mathrm{z}}$, for a total bunch charge of 0.333 nC. . . . . . . . . . . . . . . . . 135

5.19 Experimental beam dynamics results of $\epsilon_{\mathrm{n}, \mathrm{rms}}$ versus $\mathrm{Q}_{\mathrm{t}}$. . . . . 136

$5.20 \epsilon_{\mathrm{n}, \mathrm{rms}}$ versus $\theta_{\mathrm{o}} \ldots \ldots \ldots \ldots \ldots \ldots \ldots \ldots$

5.21 RMS energy spread. . . . . . . . . . . . . . . . 138

$5.2295 \%$ electron bunch length, $\tau_{95 \%}$, as a function of laser injection phase, $\theta_{0} .139$

5.23 Beam-let profile on cathode; the distance from beam-let 1 to beam-lets $2-9$ is $1 \mathrm{~mm}$. . . . . . . . . . . . . . . . . 140

$5.24 \mathrm{rf}$ gun in the symmetrized and desymmetrized mode of operation. . . 141

5.25 Profile of the point to point focused electron beam-lets with rf gun in both the symmetrized and desymmetrized mode of operation. . . . . 142

5.26 Individual beam-let radial extent. . . . . . . . . . . . . . . 143

5.27 Fourier series fit to the data in Fig. 5.26. . . . . . . . . . . . . . . 144

5.28 Eight-fold symmetric beam-lets. . . . . . . . . . . . . . 146

$5.29 \delta \theta$ versus $\mathrm{B}_{\mathrm{z}_{\mathrm{o}}} \ldots \ldots \ldots \ldots \ldots \ldots \ldots \ldots$

5.30 High energy $R_{\text {rms }}$ versus $B_{z(z=0)} \ldots \ldots \ldots$. . . . . . . . 148

5.31 Bunch length, $\tau_{95 \%}$, versus green laser intensity, $\mathrm{E}_{\gamma}$, and total electron bunch charge, $Q_{t} \ldots \ldots \ldots \ldots . \ldots \ldots$

6.1 A non-invasive feedback system to correct for the 2-D variation of the electron charge distribution. . . . . . . . . . . . . . 159 
Chapter 1

\section{Introduction}




\section{$1.1 \quad$ Introduction}

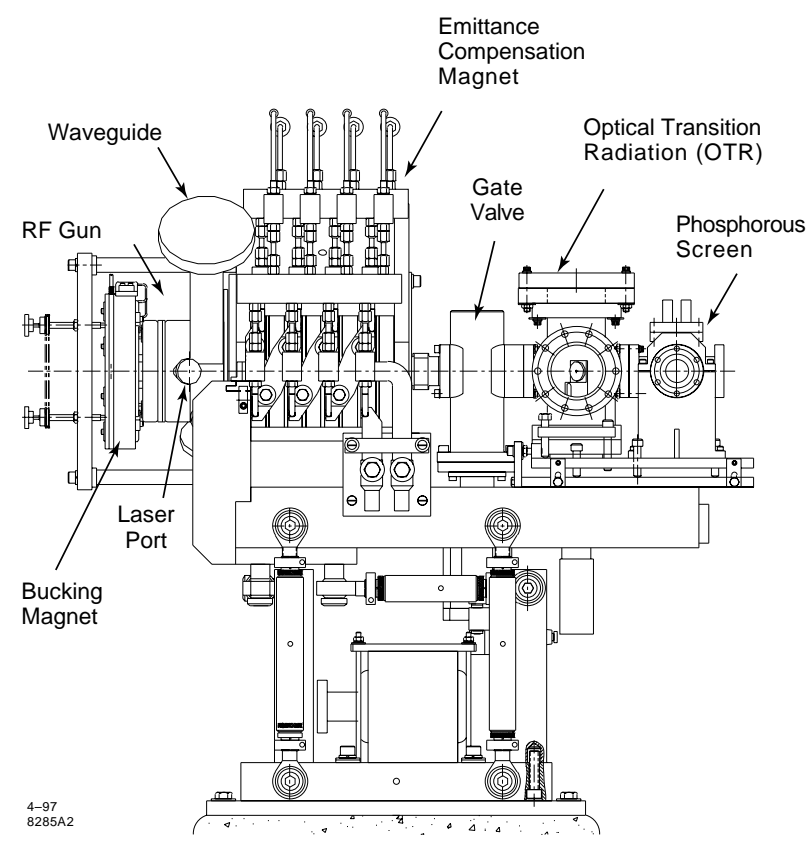

Figure 1.1: The Next Generation Photoinjector.

The need for a high-brightness electron source for the proposed Linear Coherent Light Source (LCLS) [1] at the Stanford Linear Accelerator Center (SLAC) was the driving force behind the development of the Next Generation Photoinjector (NGP). The NGP, see Fig. 1.1, consists of the BNL/SLAC/UCLA 1.6 cell S-band photocathode rf gun and single emittance compensation [2] magnet. Two major design philosophies are incorporated into the NGP: symmetry and high accelerating field gradients.

Symmetrization of all cavity wall perturbations is incorporated into the 1.6 cell $\mathrm{rf}$ gun design. This rf design philosophy decreases the dipole mode contribution to the transverse normalized rms emittance. The single emittance compensation magnet design also uses symmetry in its design, in both the longitudinal and transverse dimension.

The second design philosophy of high field gradients was driven by PARMELA [3] simulations. These simulations indicate that the best beam quality is achieved at a 
field gradient of $\mathrm{E}_{\mathrm{z}}=143 \frac{\mathrm{MV}}{\mathrm{m}}$. Most photoinjectors operate at a much lower accelerating field gradient than that required by our PARMELA simulations. The desire to operate the NGP at these higher field gradients and also to have the capability of replacing the photocathode material drove the NGP design away from a replaceable cathode plug in favor of using a demountable cathode plate.

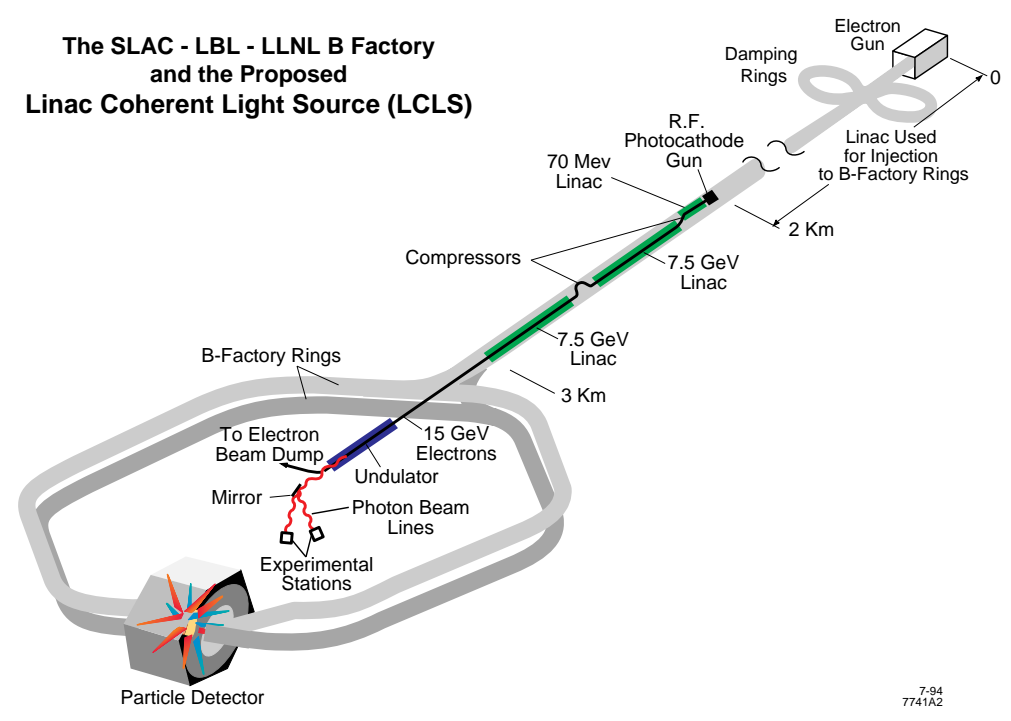

Figure 1.2: Artist's view of the proposed Linear Coherent Light Source (LCLS) at the Stanford Linear Accelerator Center.

The NGP is meant to provide a single bunch of electrons at a total charge of 1 $\mathrm{nC}$ with a transverse normalized emittance less than $1 \pi \mathrm{mm}$ mrad into a booster linac that will accelerate the electron bunch until the transverse space charge forces are eliminated by relativistic effects. Further acceleration and bunch compression is accomplished so that the desired beam parameters are attained for a Self-Amplified Spontaneous Emission (SASE) X-ray Free Electron Laser (FEL). A graphic illustration of the LCLS superimposed on the SLAC linac is shown in Fig. 1.2.

\subsection{Emittance Compensation Modeling}

Since the initial development of the theory of emittance compensation by Carlsten in 1989 [2], there has been considerable work in both the experimental and theoretical 
areas concerning the production, transport, and preservation of space charge dominated beams. The theoretical work culminated in a seminal paper by Rosenzweig and Serafini [4].

Performance of the Next Generation Photoinjector (NGP) in the emittance compensation regime is simulated using the beam dynamics code PARMELA [3]. The electro-magnetic field map used by PARMELA that models the 1.6 cell S-band rf gun was produced by SUPERFISH [5]. A magneto-static field map also used by PARMELA of the emittance compensation solenoidal magnet was produced by POISSON. Overviews of SUPERFISH, POISSON, and PARMELA are provided in the following subsections.

\subsubsection{SUPERFISH/POISSON}

The SUPERFISH [5]/POISSON family of codes are grouped together because they utilize common programs and some common subroutines to solve Maxwell's equation, Eq. 1.1, with appropriate material properties and boundary conditions, in either rectangular or cylindrically symmetric coordinates.

$$
\begin{aligned}
& \nabla \cdot \mathbf{D}=4 \pi \rho \\
& \nabla \cdot \mathbf{B}=0 \\
& \nabla \times \mathbf{H}-\frac{1}{c} \frac{\partial \mathbf{D}}{\partial \mathrm{t}}=\frac{4 \pi}{\mathrm{c}} \mathbf{J} \\
& \nabla \mathrm{x} \mathbf{E}+\frac{1}{c} \frac{\partial \mathbf{B}}{\partial \mathrm{t}}=0
\end{aligned}
$$

The initial setup of either a magnet or rf cavity problem uses three common programs, AUTOMESH, LATTICE, and VGAPLOT. AUTOMESH and LATTICE are used to generate the mesh for a given problem. VGAPLOT provides a graphical output for either SUPERFISH or POISSON problems.

When the mesh has been generated and the appropriate materials and boundary conditions have been specified, the finite difference equations are set up and solved by one of the equation-solving programs. POISSON is used for magnet problems and SUPERFISH for rf cavity problems. 
POISSON solves these finite difference equations by the method of successive point over-relaxation. It is well know that the successive point over-relaxation method converges slowly or not at all for some problems of interest. In these cases, the program PANDIRA should be used. PANDIRA uses a sparse matrix direct method to solve this set of finite difference equations. The fact that the mesh is topologically regular means that the coefficient matrix of the finite difference equations has an identical structure for any problem. Because the matrix structure is always the same, a very efficient sparse matrix direct method is used to solve this system of equations, which typically contains many thousands of equations.

SUPERFISH uses this same sparse matrix direct method to solve for the eigenvalue problem to determine the resonant frequencies in a standing-wave rf cavity.

VGAPLOT can be used to view the electro-magnetic fields produced by SUPERFISH or the magneto-statics field produced by POISSON.

\subsubsection{PARMELA}

The beam dynamics code PARMELA, supplied by the Los Alamos code group, was used to simulate the emittance compensation process of the Next Generation Photoinjector (NGP) installed on the Brookhaven Accelerator Test Facility (ATF). The acronym PARMELA stand for "Phase and Radial Motion in Electron Linacs".

PARMELA utilizes a user defined input file, input_file, to model the beam line to be simulated. PARMELA writes to an ascii text file, outpar, at the end of each space charge impulse and/or beam line element. The graphics program PARGRAF is used to view the particles distribution in phase space at the end of each element of the beam line. PARGRAF also utilizes a user defined input file, simple, to determine the beam line element at which graphics output is requested. PARGRAF outputs an ascii text file, outgraf, of the beam emittance at the end of each requested element.

PARMELA has the ability to produce both transverse and longitudinal space charge impulses to the macro particles of the electron beam at phase steps determined by input_file. The space charge impulses are calculated in the rest frame of 
the reference particle. A relativistic boost is then calculated to transform the electrostatic field of the electron bunch from the rest frame of the reference particle to the laboratory frame. The major flaw with PARMELA is that not all of the macro particles are in the rest frame of the reference particle.

One of two methods can be used to calculate the space charge impulse: point to point or the ring method. The point-to-point method uses Coulomb's law to calculate the space charge impulse for each individual macro particle due to the other n-1 macro particles. Where $\mathrm{n}$ is the total number of macro particles in the simulation. The ring method uses a Green's function approach to calculate a weighted space charge impulse on the macro particles in a cylindrical ring and at a position $\mathrm{z}$ in the electron bunch. The ring method was used in our simulations to minimize the computational time required for our simulation program. The point to point method is inherently noisy and was not used for this reason. Image charge effects of the electron near the photocathode are accounted for in both methods used for the space charge calculation. The electron bunch space charge mesh has no maximum value, but we limited ourself to a maximum of 20 grid lines in the radial direction and 400 in the longitudinal direction. When determining the radial and longitudinal extent of the space charge mesh, care must be used to insure that the electron beam is fully enveloped by the moving space charge mesh.

We model two different types of longitudinal laser profiles, Gaussian and flattop. The initial electron bunch transverse and longitudinal profile was modeled using multiple INPUT 9 cards with a Gaussian longitudinal profile with a phase offset to model a longitudinal flat-top electron beam. To model the Gaussian longitudinal laser profile, only one INPUT 9 card was used with a zero phase offset. For both longitudinal cases the transverse profile was modeled using a truncated Gaussian with $\mathrm{R}_{\max } \ll \sigma_{\mathrm{r}}$. The total number of macro particles used to model the electron bunch was 10,000. PARMELA allows the use of a maximum of 50,000 macro particles. In the middle of a simulation run, changing the space charge mesh that envelopes the electron beam and/or the amount of time/phase steps between space charge impulses, is allowed by PARMELA. 


\begin{tabular}{|l|l|}
\hline$\epsilon_{\mathrm{n}, \mathrm{rms}}$ & $1 \pi \mathrm{mm} \mathrm{mrad}$ \\
\hline $\mathrm{Q}_{\mathrm{t}}$ & $1 \mathrm{nC}$ \\
\hline$\sigma_{\mathrm{z}}$ & $3 \mathrm{psec}$ \\
\hline
\end{tabular}

Table 1.1: LCLS photoinjector parameters.

\subsection{Single Pass FEL Theory}

The theoretical development of Self-Amplified Spontaneous Emission (SASE) has been conducted by numerous authors [6] [7] and is beyond the scope of this dissertation. In the following section we shall present the constraint imposed on the electron beam transverse normalized rms emittance by the coherent part of the FEL radiation. An overview of parameter studies used to model the LCLS FEL physics will also be discussed.

\subsubsection{Emittance Requirement}

The Next Generation Photoinjector (NGP) was designed as the electron source for the LCLS X-ray FEL. The LCLS is a single pass FEL operating at $1.5 \AA$. Table 1.1 lists the major beam parameter required out of the NGP.

To ensure the highest efficiency in the SASE process, the electron beam must be fully enveloped by the coherent light produced by the FEL. Therefore the electron beam transverse normalized rms emittance must be less than the emittance of the 1.5 $\AA$ light produced by the wiggler. An upper limit of the electron beam emittance can be calculated from a $1.5 \AA$ diffraction limited laser beam. Fig. 1.3 shows a diffraction limited light beam enveloping the electron beam at a waist.

Even though an undulator is an extended linear source, within the bandwidth of the coherent part of the radiation, it can be approximated by an equivalent source [8] at its center with an angular divergence given by Eq. 1.2.

$$
\theta_{\mathrm{r}}=\sqrt{\frac{2 \lambda_{\mathrm{r}}}{\lambda_{\mathrm{u}} \mathrm{N}_{\mathrm{u}}}}
$$

where $\theta_{\mathrm{r}}$ is the angular divergence of the coherent radiation from the undulator, $\lambda_{\mathrm{r}}$ is 


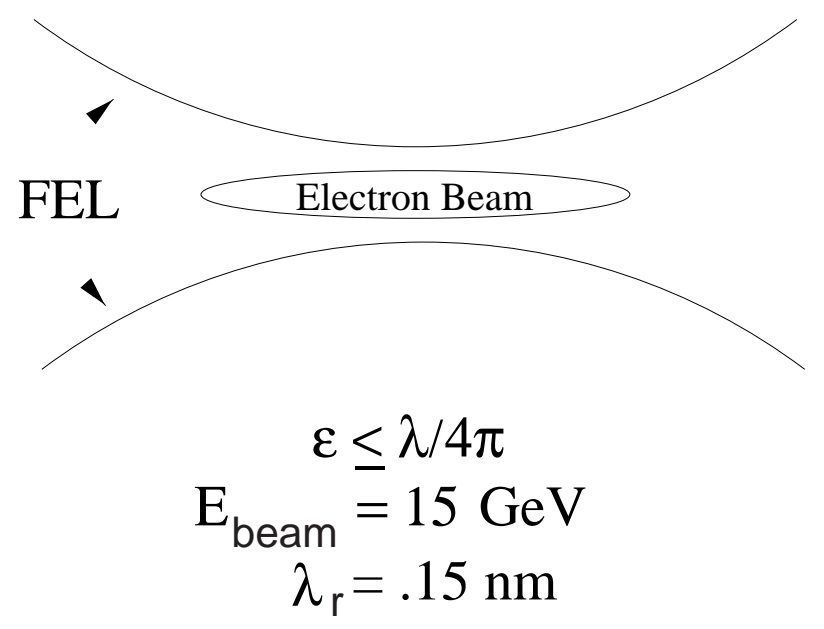

Figure 1.3: 2-D view of the overlap of the electron and FEL beams.

the wavelength of the undulator radiation, $\lambda_{\mathrm{u}}$ is the undulator period, and $\mathrm{N}_{\mathrm{u}}$ is the number of undulator periods.

The effective source radius, $\sigma_{\mathrm{r}}$, of the coherent part of the radiation from the undulator [8] is given by Eq. 1.3.

$$
\sigma_{\mathrm{r}}=\frac{1}{4 \pi} \sqrt{\frac{\lambda_{\mathrm{r}} \lambda_{\mathrm{u}} \mathrm{N}_{\mathrm{u}}}{2}}
$$

Combing the results from Eq. 1.2 and 1.3, we find that the emittance of the coherent part of the FEL radiation [8] is given by Eq 1.4.

$$
\epsilon_{\mathrm{r}}=\sigma_{\mathrm{r}} \theta_{\mathrm{r}}=\frac{\lambda_{\mathrm{r}}}{4 \pi}
$$

For the electron beam to be fully enveloped by the coherent part of the FEL radiation, the transverse normalized rms emittance, $\epsilon_{\mathrm{n}, \mathrm{rms}}$, must be less than or on the same order as the optical beam, Eq. 1.5. This relationship sets the electron beam transverse normalized rms emittance requirement.

$$
\epsilon_{\mathrm{n}, \mathrm{rms}} \leq \beta \gamma \epsilon_{\mathrm{r}}
$$




\subsubsection{FEL Parameter Optimization}

The results of SASE parameter studies [9] are presented in the context of transverse emittance as it pertains to the saturated power level of an SASE FEL. The output power of an SASE FEL can be characterized by Eq. 1.6.

$$
\mathrm{P}=\alpha_{\mathrm{c}} \mathrm{P}_{\mathrm{n}} \mathrm{e}^{\frac{z}{\mathrm{Lg}_{\mathrm{g}}}} \leq \mathrm{P}_{\mathrm{sat}},
$$

where $\alpha_{c}$ is the coupling coefficient representing the fraction of the noise power $\mathrm{P}_{\mathrm{n}}$ coupled into the dominant mode that is growing exponentially along the wiggler in the $\mathrm{z}$ direction, whose gain length is represented by $\mathrm{L}_{\mathrm{g}}$, and $\mathrm{P}_{\mathrm{sat}}$ is the saturation power limit defined in Eq. 1.7, which scales like $\frac{\mathrm{L}_{1 d}}{\mathrm{~L}_{\mathrm{g}}}$.

$$
\mathrm{P}_{\mathrm{sat}} \approx 1.6 \rho_{\mathrm{P}}\left(\frac{\mathrm{L}_{1 \mathrm{~d}}}{\mathrm{~L}_{\mathrm{g}}}\right)^{2} \mathrm{P}_{\text {beam }}
$$

where $\rho_{\mathrm{P}}$ is the dimensionless Pierce parameter. Since the ratio of 1-D gain length, $\mathrm{L}_{1 \mathrm{~d}}$, to the power gain length, $\mathrm{L}_{\mathrm{g}}$, is an important ratio of the SASE FEL process,

a universal scaling function $\mathcal{F}$ has been defined. $\frac{\mathrm{L}_{1 d}}{\mathrm{~L}_{\mathrm{g}}}$ is an implicit function and has been parameterized by Eq. 1.8.

$$
\frac{\mathrm{L}_{1 \mathrm{~d}}}{\mathrm{~L}_{\mathrm{g}}}=\mathcal{F}\left(\eta_{\mathrm{d}}, \eta_{\epsilon}, \eta_{\gamma}\right)=\frac{1}{1+\eta},
$$

where

$$
\begin{aligned}
\eta= & a_{1} \eta_{d}^{a_{2}}+a_{3} \eta_{\epsilon}^{a_{4}}+a_{5} \eta_{\gamma}^{a_{6}}+ \\
& a_{7} \eta_{\epsilon}^{a_{8}} \eta_{\gamma}^{a_{9}}+a_{10} \eta_{d}^{a_{11}} \eta_{\gamma}^{a_{12}}+a_{13} \eta_{d}^{a_{14}} \eta_{\epsilon}^{a_{15}}+ \\
& a_{16} \eta_{d}^{a_{17}} \eta_{\epsilon}^{a_{18}} \eta_{\gamma}^{a_{19}}
\end{aligned}
$$

and where $\eta_{d}, \eta_{\epsilon}$, and $\eta_{\gamma}$ are given below in Eq. 1.10, Eq. 1.11, and Eq. 1.12. The 19 fitting parameters are presented in Table 1.2.

$$
\eta_{\mathrm{d}}=\frac{\mathrm{L}_{1 \mathrm{~d}}}{\mathrm{~L}_{\mathrm{r}}}
$$

where $L_{r}$ is the Rayleigh range. 


\begin{tabular}{|l|l|l|l|}
\hline$a_{1}=0.45$ & $a_{2}=0.57$ & $a_{3}=0.55$ & $a_{4}=1.6$ \\
\hline$a_{5}=3$ & $a_{6}=2$ & $a_{7}=0.35$ & $a_{8}=2.9$ \\
\hline$a_{9}=2.4$ & $a_{10}=51$ & $a_{11}=0.95$ & $a_{12}=3$ \\
\hline$a_{13}=5.4$ & $a_{14}=0.7$ & $a_{15}=1.9$ & $a_{16}=1140$ \\
\hline$a_{17}=2.2$ & $a_{18}=2.9$ & $a_{19}=3.2$ & \\
\hline
\end{tabular}

Table 1.2: Universal scaling law fit parameters.

$$
\eta_{\epsilon}=\left(\frac{\mathrm{L}_{1 \mathrm{~d}}}{\beta_{\mathrm{T}}}\right)\left(\frac{4 \pi \epsilon}{\lambda}\right)
$$

where $\beta_{\mathrm{T}}$ is a Twiss parameter, $\epsilon$ is the beam transverse emittance, and $\lambda$ is the FEL wavelength.

$$
\eta_{\gamma}=\left(\frac{\mathrm{L}_{1 \mathrm{~d}}}{\lambda_{\mathrm{w}}}\right)\left(\frac{\sigma_{\mathrm{e}}}{\mathrm{E}_{\text {beam }}}\right)
$$

where $\lambda_{\mathrm{w}}$ is the wiggler period, $\sigma_{\mathrm{e}}$ is the rms energy spread of the electron beam, and $\mathrm{E}_{\text {beam }}$ is the average beam energy.

From Eq. 1.8, the shortest gain length will be attained when $\eta$ is zero. The terms $\eta_{d}, \eta_{\epsilon}$, and $\eta_{\gamma}$ are a measure of the difference between the 1-D model and a real beam, where $\eta_{d}$ is the gain length reduction due to diffraction, and $\eta_{\epsilon}$ and $\eta_{\gamma}$ are the gain length reduction due to the electron beam's longitudinal velocity spread due to emittance and energy spread, respectfully.

\subsection{Electron Sources}

The first question that came to mind when the 1.6 cell symmetrized rf gun development project was started was: "Is there an electron source capable of producing the necessary electron beam parameters required for the LCLS project, namely, $1 \mathrm{nC}$ and $1 \pi \mathrm{mm}$ mrad normalized rms emittance?". In this section we shall present the state of the art in electron sources from DC thermionic guns to rf photocathode guns and discuss their virtues and drawbacks. 


\subsubsection{Electron Sources}

\section{Thermionic DC Electron Sources}

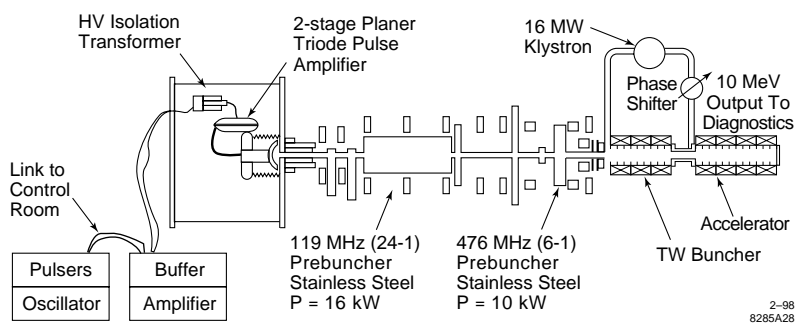

Figure 1.4: Boeing Corporation thermionic DC injector.

In the early 1980s, the Boeing Corporation developed a high current injector for FEL experiments [10]. The Boeing injector is graphically illustrated in Fig. 1.4. It consists of a DC thermionic gun and two stages of sub-harmonic bunching. The first stage operated at the $119 \mathrm{MHz}, 24$ th sub-harmonic of $2856 \mathrm{MHz}$ and the second stage at $476 \mathrm{MHz}$, which is the 6th sub-harmonic of the linac frequency.

The Boeing electron source is capable of producing the necessary charge but not the longitudinal pulse length for the LCLS. Also the transverse rms emittance was measured to be $6.4 \pi \mathrm{mm}$ mrad, a factor of six larger than the LCLS beam requirements. The pertinent beam parameters attained by the Boeing injector are given in Table 1.3.

\begin{tabular}{|l|l|}
\hline $\mathrm{Q}_{\mathrm{t}}$ & $1.2 \mathrm{nC}$ \\
\hline$\epsilon_{\mathrm{n}, \mathrm{rms}}$ & $6.4 \pi \mathrm{mm} \mathrm{mrad}$ \\
\hline$\sigma_{\mathrm{z}}$ & $5 \mathrm{psec}$ \\
\hline$\gamma$ & 20 \\
\hline
\end{tabular}

Table 1.3: Electron beam parameters for the Boeing thermionic DC gun/injector system.

\section{Photocathode DC Electron Sources}

The electron source for the high energy physics program at SLAC requires a spin polarized electron beam [11]. This is accomplished by using a circular polarized laser 


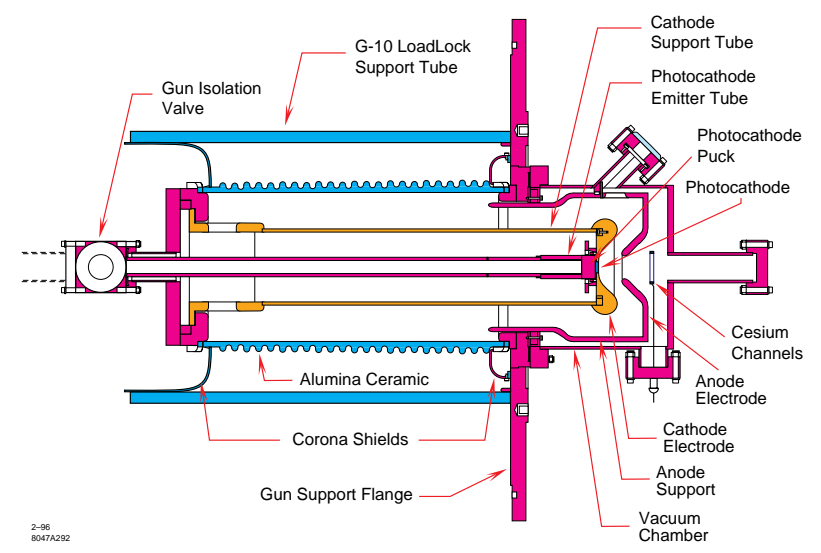

Figure 1.5: SLAC polarized photocathode DC gun.

tuned to the direct band gap of GaAs. The accelerating field of the gun has a large DC potential, like that used in the Boeing electron injector.

The SLAC polarized DC gun is graphically illustrated in Fig. 1.5. The SLAC polarized DC gun along with a S-band sub-harmonic bunching system is required to produce the beam parameters in Table 1.4.

\begin{tabular}{|l|l|}
\hline $\mathrm{Q}_{\mathrm{t}}$ & $8 \mathrm{nC}$ \\
\hline$\epsilon_{\mathrm{n}, \mathrm{rms}}^{\mathrm{x}, \mathrm{y}}$ & $100 \pi \mathrm{mm} \operatorname{mrad}$ \\
\hline$\sigma_{\mathrm{z}}$ & $5 \mathrm{psec}$ \\
\hline$\gamma$ & 80 \\
\hline
\end{tabular}

Table 1.4: SLAC polarized DC gun/injector system.

Even if the total charge is decreased from $8 \mathrm{nC}$ down to the $1 \mathrm{nC}$ limit, the transverse emittance requirement for the LCLS injector cannot be satisfied by the SLAC polarized source. This is assuming an emittance charge scaling of $\epsilon_{\mathrm{n}, \mathrm{rms}} \propto \mathrm{Q}_{\mathrm{t}}^{\frac{2}{3}}[12]$.

\subsection{2 rf Electron Sources}

The time structure of an electron source based on $\mathrm{rf}$ accelerating gradients is determined by the frequency of the rf power source. At S-band, the maximum electron bunch length is on the order of $10 \mathrm{psec}$ or $10^{\circ}$ of $\mathrm{rf}$ phase. This requirement is 
determined by the desire to minimize the nonlinear rf emittance contribution caused by the time dependent radial forces and to eliminate the need of any type of bunching systems. In this section we will present two types of rf based electron sources, the thermionic and photocathode rf guns.

\section{Thermionic rf Electron Sources}

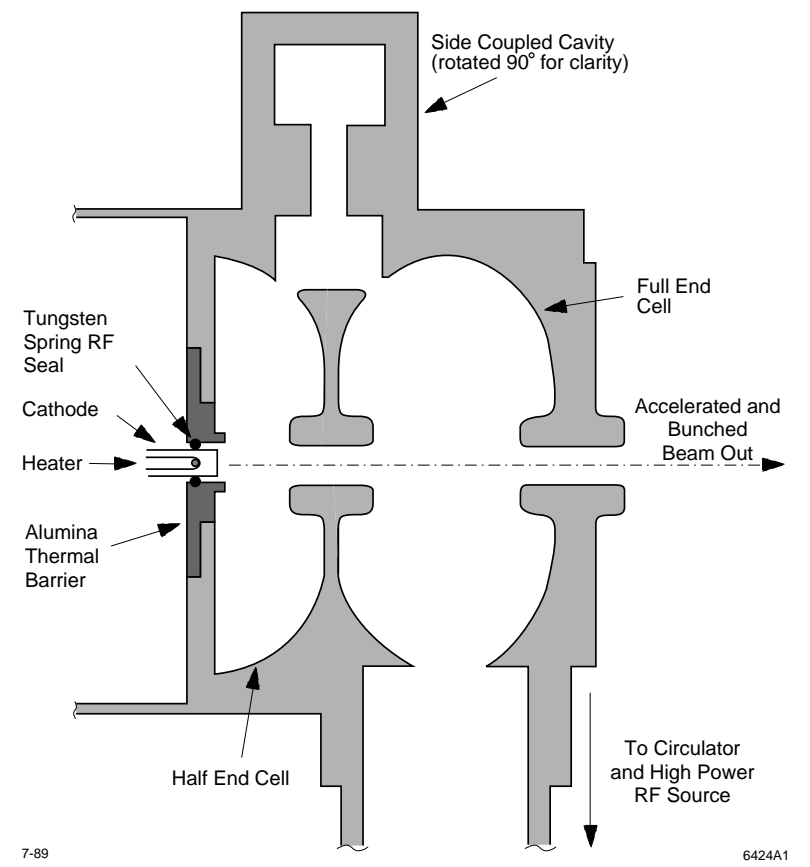

Figure 1.6: SSRL thermionic rf gun.

The electron source for the Stanford Synchrotron Radiation Laboratory (SSRL) is a thermionic rf gun [13]. This rf gun is graphically illustrated in Fig. 1.6. The thermionic rf electron injector which consists of a thermionic rf gun, alpha magnet, and rf chopper is capable of producing a very short, low charge electron bunch. The transverse normalized rms emittance, $\epsilon_{\mathrm{n}, \mathrm{rms}}$, of thermionic electron injector will not meet the beam requirements of the LCLS electron injector. The beam parameters that the SSRL thermionic rf electron injector is capable of producing are listed in Table 1.5. It should be noted that the thermionic rf gun in combination with an alpha magnet is capable of producing an ultra short electron bunch on the order of a 
50 fsec [14].

\begin{tabular}{|l|l|}
\hline $\mathrm{Q}_{\mathrm{t}}$ & $0.3 \mathrm{nC}$ \\
\hline$\epsilon_{\mathrm{n}, \mathrm{rms}}^{\mathrm{x}, \mathrm{y}}$ & $30 \pi \mathrm{mm} \mathrm{mrad}$ \\
\hline$\sigma_{\mathrm{z}}$ & $1 \mathrm{psec}$ \\
\hline$\gamma$ & 4 \\
\hline
\end{tabular}

Table 1.5: SSRL thermionic rf gun/injector system.

\section{Photocathode RF Electron Sources}

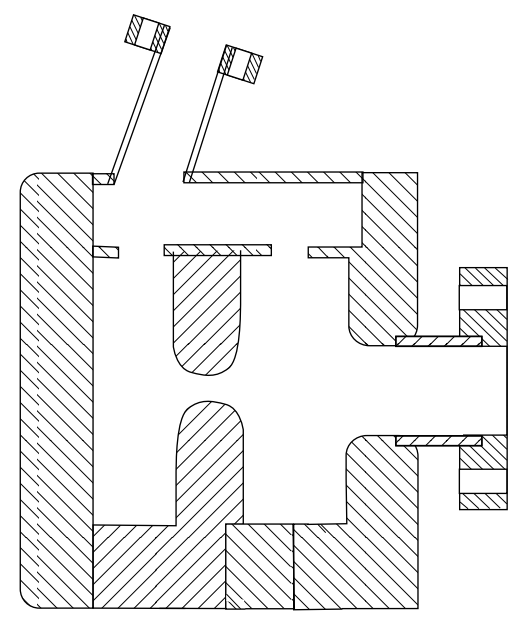

Figure 1.7: BNL 1.5 cell side coupled zero mode suppressed photocathode rf gun.

The advent of the photocathode rf gun [15] has brought the goal of $1 \mathrm{nC}$ and $1 \pi$ mm mrad electron beam into the realm of possibility. Illustrated in Fig. 1.7 is a side coupled one and half cell Brookhaven style photocathode rf gun [16].

The beam parameters that the BNL side-coupled rf gun is capable of producing are listed in Table 1.6. The transverse normalized rms beam emittance, $\epsilon_{\mathrm{n}, \mathrm{rms}}$, is a factor of two and a half larger than that required by the LCLS injector. The BNL type rf gun is the starting point of the NGP project. It was determined that this type of electron source would be capable of producing the required beam quality if the rf dipole mode were eliminated in the NGP design. 
The remainder of this dissertation will deal with the design, construction, rf testing, magnet design, and beam dynamics studies of the Next Generation Photoinjector.

\begin{tabular}{|l|l|}
\hline $\mathrm{Q}_{\mathrm{t}}$ & $1 \mathrm{nC}$ \\
\hline$\epsilon_{\mathrm{n}, \mathrm{rms}}^{\mathrm{x},}$ & $2.5 \pi \mathrm{mm} \operatorname{mrad}$ \\
\hline$\sigma_{\mathrm{z}}$ & $4.5 \mathrm{psec}$ \\
\hline$\gamma$ & 80 \\
\hline
\end{tabular}

Table 1.6: BNL/ATF Photocathode rf gun/injector system.

\subsection{Summary}

The four major electron sources discussed in Section 1.4 do not meet the requirements of the LCLS electron source. The BNL type photocathode rf gun comes closest to the beam requirements of the LCLS SASE FEL, except in the area of transverse emittance, where it is a factor of two and a half larger than the required $1 \pi \mathrm{mm} \operatorname{mrad}$. Calculation [17] and simulation [18] indicate that if the rf dipole mode contribution to the transverse emittance is eliminated, then the photocathode rf electron gun is capable of producing the beam parameters required for the LCLS SASE FEL.

The remainder of this dissertation deals with the design, simulation, production, low level rf testing, and experimental beam dynamics studies of the Next Generation Photoinjector (NGP). 
Chapter 2

Beam Dynamics: Theory and Simulations 


\section{$2.1 \quad$ Introduction}

In this chapter we shall lay the groundwork for the theoretical development of emittance compensated photoinjectors. To accomplish this, an understanding of transverse phase space beam dynamics theory will be necessary. The individual emittance contribution terms will be calculated. This allows for the estimation of the electron beam's normalized rms emittance, $\epsilon_{\mathrm{n}, \mathrm{rms}}$, in the limit of zero charge, which was found to be between $0.68 \pi \mathrm{mm} \mathrm{mrad} \leq \epsilon_{\mathrm{n}, \mathrm{rms}} \leq 1.13 \pi \mathrm{mm} \mathrm{mrad}$. This is in agreement with our experimental data in Section 5.6 .2 of $\lim _{\mathrm{Q}_{\mathrm{t}} \rightarrow 0} \epsilon_{\mathrm{n}, \mathrm{rms}}=0.8 \pi \mathrm{mm} \mathrm{mrad}$. These results are dominated by the thermal emittance term, $\epsilon_{\mathrm{o}}$, which our theoretical prediction indicates is equal to $\epsilon_{\mathrm{o}}=0.62 \pi \mathrm{mm}$ mrad. Simulation results using PARMELA [3] with field maps from SUPERFISH [5] and POISSON are presented using both Gaussian and flat-top longitudinal laser pulses for a host of parameters. When PARMELA [3] is compared to actual experiments, there are indications that it does not accurately model physical reality.

\subsection{Transverse Linear Space Charge Emittance Compensation}

In 1987 Carlsten [2] proposed a scheme to align the different slices of the electron beam in such a fashion as to minimize the integrated transverse normalized rms emittance, $\epsilon_{\mathrm{n}, \mathrm{rms}}$, of the beam. Carlsten's emittance compensation process is graphically represented [19] in Fig. 2.1.

The evolution of the emittance compensation process can be described in four steps. The beam is assumed to have a uniform distribution in both the transverse and longitudinal dimensions.

In Fig. 2.1, sub-plot 1, the transverse phase space of the electron beam is shown with a zero emittance. As the beam drifts from position 1 to 2 the radial space charge forces cause the integrated transverse phase space area to increase, with the radius of the core increasing a factor of two greater than that of the head and tail of the bunch. This space charge force expansion of the electron beam phase space is represented in 

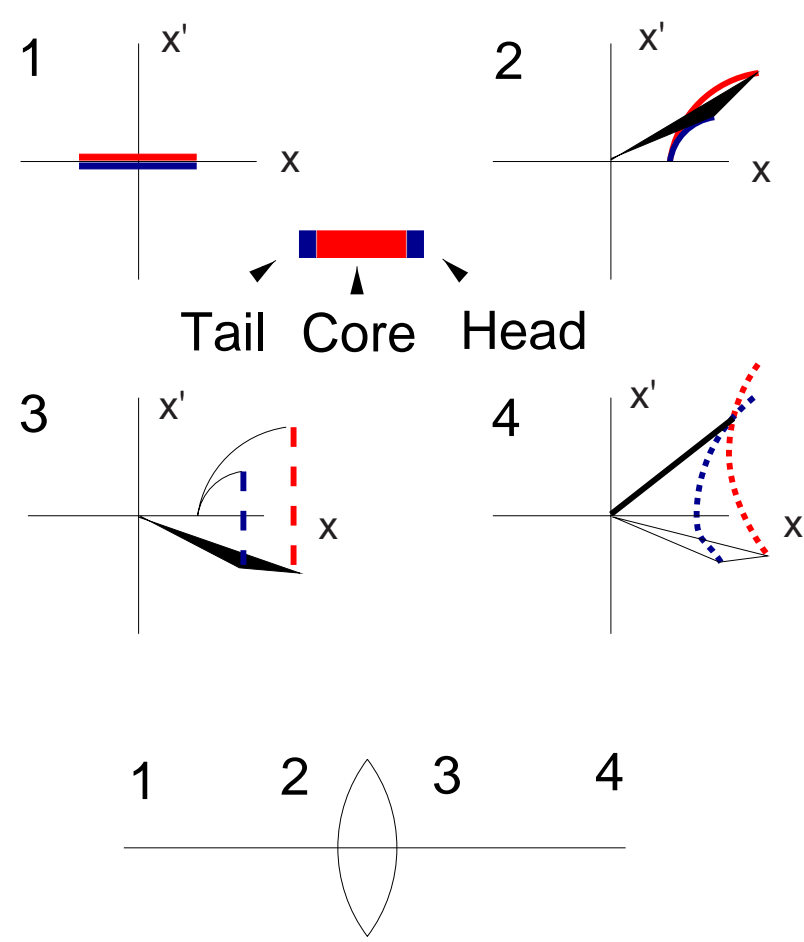

Figure 2.1: Graphically representation of Carlsten's emittance compensation process. 
sub-plot 2 of Fig. 2.1. As the zero energy spread electron beam travels through the thin lens solenoidal focusing field, the individual slices of the electron bunch receive the same focusing kick. In an individual slice this kick is proportional to the electron $\mathrm{x}$ position. This is represented by sub-plot 3 in Fig. 2.1. Since the beam is now convergent and the space charge forces are defocusing as the beam drifts from point 3 to 4 , the individual slices of the electron beam will follow the phase space trajectory shown in sub-plot 4 of Fig. 2.1. At some point $z_{0}$ in the drift region, the head, tail, and core realign into a zero emittance beam. In this basic model of the emittance compensation process the beam's projected transverse phase space will again start to grow since the transverse space charge forces have not been eliminated.

If the drift space is replaced by an acceleration section such that the point $z_{0}$ is at a sufficiently high energy, the transverse space charge forces of the electron bunch will be eliminated by the $\frac{1}{\gamma^{2}}$ effect. Consequently the beam's low transverse emittance is frozen in. For the Linear Coherent Light Source (LCLS) photoinjector, it was found that a beam energy of $150 \mathrm{MeV}$ was sufficient to eliminate the transverse space charge forces and freeze in $\epsilon_{\mathrm{n}, \mathrm{rms}}[20]$.

This simple model of the emittance compensation process does not include the coupling of the acceleration in the gun with focusing of the compensation solenoid in the same physical space, and the post acceleration to a high enough energy to eliminate the transverse space charge force. These issues as they pertain to the 1.6 cell gun and single emittance compensation magnet were studied using PARMELA [3]. The electro-magnetic fields of the rf gun and the magneto-static field of the compensation magnet were generated using SUPERFISH [5] and POISSON. These fields were used by PARMELA [3] to model the beam dynamics of the emittance compensation process numerically.

The generalization of Carlsten's model to the case of a beam which is accelerated after the drift space was developed by Serafini and Rosenzweig [4]. Their approach is based on the rms beam envelope equation to describe the propagation of each slice of the electron bunch. A solution to the rms envelope equation was found that performs emittance correction. This model is called the invariant envelope (IE). Analytical predictions of the photoinjector settings needed to achieve emittance compensation 
under the IE are also derived by Serafini and Rosenzweig. The IE is characterized by a beam radius, $\mathrm{R}_{\mathrm{IE}}$, scaling as in Eq. 2.1

$$
\mathrm{R}_{\mathrm{IE}} \propto \frac{1}{\gamma^{\prime}} \sqrt{\frac{\mathrm{I}_{\mathrm{p}}}{\gamma}}
$$

where $\mathrm{I}_{\mathrm{p}}$ is the peak current of the electron bunch, $\gamma$ and $\gamma^{\prime}$ are defined in Eq. 2.2.

$$
\gamma=\frac{1}{\sqrt{1-\beta^{2}}} \quad \gamma^{\prime}=\frac{\mathrm{d} \gamma}{\mathrm{dz}} \quad \beta=\frac{\mathrm{v}}{\mathrm{c}}
$$

where $\mathrm{v}$ is the velocity of an electron, $\mathrm{c}$ is the speed of light, and $\mathrm{z}$ is the longitudinal position coordinate down the beam line.

This implies that at some energy down the beam acceleration and transport system, the laminar flow, which is fully space charge dominated, will enter the emittance dominated regime. Here incoherent betatron motion is dominant over the collective space charge beam oscillation. At this point the emittance correction process is halted and the rms normalized emittance is invariant. A useful parameter which states when the beam is still in the laminar flow regime and additional emittance compensation is still possible is given in Eq. 2.3 when $\rho_{\mathrm{IE}}>>1$.

$$
\rho_{\mathrm{IE}}=\frac{\left\langle\mathrm{g}^{2}\right\rangle}{\sqrt{1+4 \Omega^{2}}}\left[\frac{\mathrm{I}_{\mathrm{p}} / \mathrm{I}_{\mathrm{A}}}{\gamma \epsilon_{\mathrm{n}, \mathrm{rms}} \gamma^{\prime}}\right]^{2}
$$

where $I_{p}$ is the peak current of the beam, $I_{A}$ is the Alfvén current which is equal to $17 \mathrm{kA}, \Omega^{2}$ is the normalized focusing frequency $\left(\Omega^{2} \cong \frac{1}{8}\right.$ in the standing wave linacs and $\Omega^{2} \cong 0$ in a traveling wave linacs), $\epsilon_{\mathrm{n}, \mathrm{rms}}$ is the thermal emittance of the beam, $\left\langle\mathrm{g}^{2}\right\rangle$ is the rms average of the transverse space charge field form factor $\left(\left\langle\mathrm{g}^{2}\right\rangle=1\right.$ for a uniform current distribution and $\left\langle\mathrm{g}^{2}\right\rangle=\frac{1}{\sqrt{3}}$ for a Gaussian beam), $\gamma$ is the normalized beam energy, and $\gamma^{\prime}$ is the accelerating gradient. 


\subsection{Beam Dynamics Theory}

\subsubsection{Liouville's Theorem}

Liouville's Theorem [21] states that if a system of particles is in a conservative force field the density of the particles in phase space stays constant. This is graphically represented in Fig. 2.2, in which we see the phase space of a collection of particles evolve in time from one configuration to another. Although the shape of the phase space changes, we will prove that the density of the phase space is a constant. In this section we shall prove this theorem using a Hamiltonian dynamics approach.

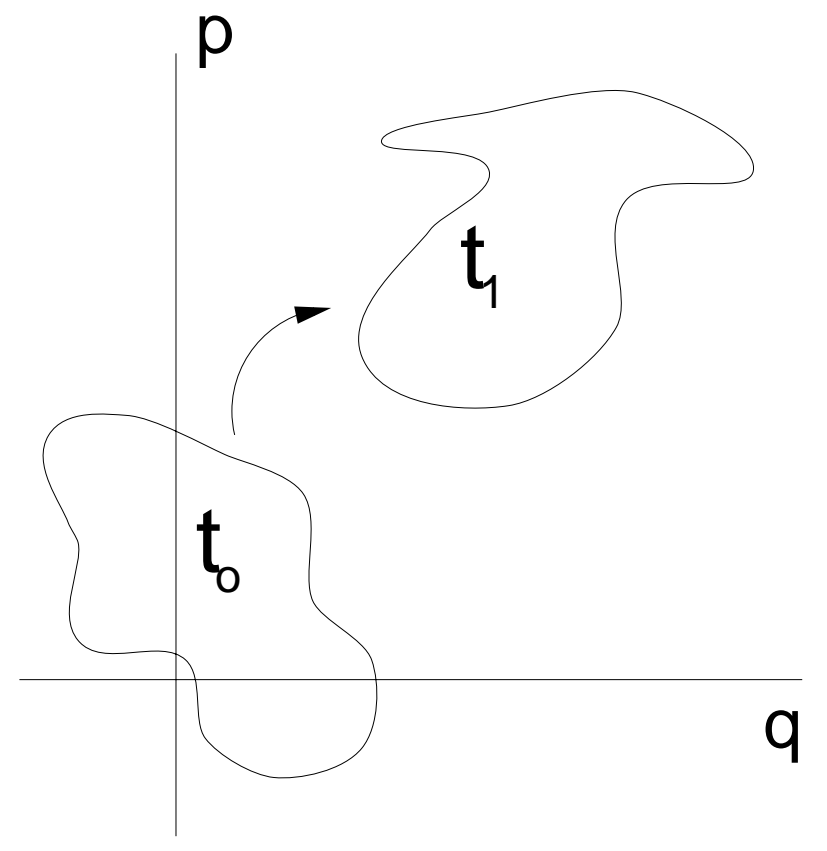

Figure 2.2: Phase space density as a function of time.

We want to prove that the density of phase space is constant, which implies that the total time derivative of the phase space density is zero.

$$
\begin{gathered}
\rho=\rho\left(\mathrm{q}_{\mathrm{i}}, \mathrm{p}_{\mathrm{i}}, \mathrm{t}\right) \\
\frac{\mathrm{d} \rho}{\mathrm{dt}}=0
\end{gathered}
$$


Assuming that there is no particle loss or gain we can start at the equation of continuity, Eq. 2.6.

$$
\frac{\partial \rho}{\partial \mathrm{t}}+\nabla[\rho \overline{\mathbf{v}}]=0
$$

where

$$
\begin{gathered}
\overline{\mathbf{v}}=\left[\dot{\mathrm{q}}_{\mathrm{i}}, \dot{\mathrm{p}}_{\mathrm{i}}\right] \\
\frac{\partial \rho}{\partial \mathrm{t}}+\frac{\partial}{\partial \mathrm{q}_{\mathrm{i}}}\left(\rho \dot{\mathrm{q}}_{\mathrm{i}}\right)+\frac{\partial}{\partial \mathrm{p}_{\mathrm{i}}}\left(\rho \dot{\mathrm{p}}_{\mathrm{i}}\right)=0 \\
\frac{\partial \rho}{\partial \mathrm{t}}+\rho \frac{\partial \dot{\mathrm{q}}_{\mathrm{i}}}{\partial \mathrm{q}_{\mathrm{i}}}+\dot{\mathrm{q}_{\mathrm{i}}} \frac{\partial \rho}{\partial \mathrm{q}_{\mathrm{i}}}+\rho \frac{\partial \dot{\mathrm{p}_{\mathrm{i}}}}{\partial \mathrm{p}_{\mathrm{i}}}+\dot{\mathrm{p}_{\mathrm{i}}} \frac{\partial \rho}{\partial \mathrm{p}_{\mathrm{i}}}=0 \\
\frac{\partial \rho}{\partial \mathrm{t}}+\left[\dot{\mathrm{q}}_{\mathrm{i}} \frac{\partial \rho}{\partial \mathrm{q}_{\mathrm{i}}}+\dot{\mathrm{p}_{\mathrm{i}}} \frac{\partial \rho}{\partial \mathrm{p}_{\mathrm{i}}}\right]+\rho\left[\frac{\partial \dot{\mathrm{q}_{\mathrm{i}}}}{\partial \mathrm{q}_{\mathrm{i}}}+\frac{\partial \dot{\mathrm{p}_{\mathrm{i}}}}{\partial \mathrm{p}_{\mathrm{i}}}\right]=0
\end{gathered}
$$

From Hamilton's equations of motion [22] we know that

$$
\frac{\partial \mathrm{H}}{\partial \mathrm{q}_{\mathrm{i}}}=-\dot{\mathrm{p}}_{\mathrm{i}} \quad \frac{\partial \mathrm{H}}{\partial \mathrm{p}_{\mathrm{i}}}=\dot{\mathrm{q}}_{\mathrm{i}}
$$

so we can rewrite Eq. 2.10 in the following form:

$$
\begin{gathered}
\frac{\partial \rho}{\partial \mathrm{t}}+\frac{\partial \rho}{\partial \mathrm{q}_{\mathrm{i}}} \frac{\mathrm{d \textrm {q } _ { \mathrm { i } }}}{\mathrm{dt}}+\frac{\partial \rho}{\partial \mathrm{p}_{\mathrm{i}}} \frac{\mathrm{d \textrm {p } _ { \mathrm { i } }}}{\mathrm{dt}}+\rho\left[\frac{\partial^{2} \mathrm{H}}{\partial \mathrm{q}_{\mathrm{i}} \partial \mathrm{p}_{\mathrm{i}}}-\frac{\partial^{2} \mathrm{H}}{\partial \mathrm{q}_{\mathrm{i}} \partial \mathrm{p}_{\mathrm{i}}}\right]=0 \\
\frac{\partial \rho}{\partial \mathrm{t}}+\frac{\partial \rho}{\partial \mathrm{q}_{\mathrm{i}}} \frac{\mathrm{d \textrm {q } _ { \mathrm { i } }}}{\mathrm{dt}}+\frac{\partial \rho}{\partial \mathrm{p}_{\mathrm{i}}} \frac{\mathrm{dp_{ \textrm {i } }}}{\mathrm{dt}}=0
\end{gathered}
$$

which is therefore the total time derivative of the phase space density.

$$
\frac{\mathrm{d} \rho}{\mathrm{dt}}=0
$$




\subsubsection{Emittance Concepts}

Pedagogy dictates that we must define what we mean by emittance. Eq. 2.15 is used as the theoretical definition for the normalized rms emittance [23], whose units are $\pi \mathrm{mm}$ mrad.

$$
\epsilon_{\mathrm{n}, \mathrm{rms}}=\beta \gamma \sqrt{\left\langle\mathrm{x}^{2}\right\rangle\left\langle\mathrm{x}^{2}\right\rangle-\left\langle\mathrm{xx}^{\prime}\right\rangle^{2}}
$$

Where we have defined each of Eq. 2.15's terms individually in Eqs. 2.16, 2.17, and 2.18 .

$$
\begin{aligned}
\left\langle\mathrm{x}^{2}\right\rangle & =\frac{\iint \mathrm{x}^{2} \rho \mathrm{dx} d \mathrm{x}^{\prime}}{\iint \rho \mathrm{dx} \mathrm{dx}^{\prime}} \\
\left\langle\mathrm{x}^{\prime 2}\right\rangle & =\frac{\iint \mathrm{x}^{\prime 2} \rho \mathrm{dx} \mathrm{dx} \mathrm{x}^{\prime}}{\iint \rho \mathrm{dx} \mathrm{d} \mathrm{x}^{\prime}} \\
\left\langle\mathrm{xx}^{\prime}\right\rangle & =\frac{\iint \mathrm{xx}^{\prime} \rho \mathrm{dx} \mathrm{dx} \mathrm{x}^{\prime}}{\iint \rho \mathrm{dx} \mathrm{d} \mathrm{x}^{\prime}}
\end{aligned}
$$

The relationship between the transverse normalized and transverse geometrical rms emittance is given in Eq. 2.19, where $\beta$ and $\gamma$ are the relativistic factors defined in Eq. 2.2. Unless otherwise stated, all emittances discussed will be the transverse normalized rms, $\epsilon_{\mathrm{n}, \mathrm{rms}}$, in units of $\pi \mathrm{mm} \operatorname{mrad}$.

$$
\epsilon_{\mathrm{n}, \mathrm{rms}}=\beta \gamma \epsilon_{\mathrm{g}, \mathrm{rms}}
$$

The normalized rms projected emittance is not a conserved quantity in the Liouvillian sense. But it is a useful quantity since it allows one to calculate the beams envelope in a field free region.

In Eqs. 2.16, 2.17, and 2.18 the integration was implicit over all $\mathrm{z}$ and $\mathrm{z}^{\prime}$. When used with Eq. 2.19, the normalized rms emittance is also called the projected normalized rms emittance. If the integration in Eqs. 2.16, 2.17, and 2.18 are limited over a narrow band of $\mathrm{z}$ but all $\mathrm{z}^{\prime}$, this quantity is called the slice normalized rms emittance. The projected and slice emittances are defined in Eq. 2.20. 


$$
\begin{array}{ll}
\epsilon_{\mathrm{n}, \mathrm{rms}}^{\text {projected }}=\beta \gamma \epsilon_{\mathrm{g}, \mathrm{rms}}^{\text {projected }} & \text { all } \mathrm{z} \text { and } \mathrm{z}^{\prime} \\
\epsilon_{\mathrm{n}, \mathrm{rms}}^{\text {slice }}=\beta \gamma \epsilon_{\mathrm{g}, \mathrm{rms}}^{\text {llice }} & \mathrm{z}_{\mathrm{n}} \text { to } \mathrm{z}_{\mathrm{n}+1} \text { and all } \mathrm{z}^{\prime}
\end{array}
$$

\subsubsection{Emittance Terms}

Six emittance terms contribute to the electron beam's total normalized rms emittance, $\epsilon_{\mathrm{n}, \mathrm{rms}}$. These six emittance terms are discussed in detail in the following subsection.

The space charge, $\epsilon_{\mathrm{sc}}$, and $\mathrm{rf}, \epsilon_{\mathrm{rf}}$, emittance terms compete with each other with respect to the accelerating field gradient, $E_{0}$, because the space charge term scales as $\frac{1}{\mathrm{E}_{\mathrm{o}}}$ and the rf term scales as $\mathrm{E}_{\mathrm{o}}$. These scaling relationships make it necessary to perform beam dynamics simulations to find the optimum operational accelerating field gradient.

The ultimate goal of the 1.6 cell rf gun was to reduce the dipole mode in the full cell of the gun. Though this was accomplished, the residual dipole field continues to contribute an emittance growth term represented by $\epsilon_{\mathrm{mp}}$.

Since the Next Generation Photoinjector (NGP) has no bucking magnet, there is a finite longitudinal magnetic field at the cathode. Therefore the electron beam is produced with a finite angular momentum which will manifest itself as an emittance term, $\epsilon_{\mathrm{B}_{\mathrm{z}}}$.

The thermal emittance, $\epsilon_{\mathrm{o}}$, is due to the energy difference between the laser photon energy and the effective work function of the photoemitting material.

The emittance term, $\epsilon_{\mathrm{T}}$, is due to the actual physical temperature of the photoemitting material.

$$
\begin{gathered}
\sqrt{\left[\epsilon_{\mathrm{sc}}^{2}+\epsilon_{\mathrm{rf}}^{2}+\epsilon_{\mathrm{mp}}^{2}+\epsilon_{\mathrm{B}_{\mathrm{z}}}^{2}+\epsilon_{\mathrm{o}}^{2}+\epsilon_{\mathrm{T}}^{2}\right]} \\
\leq \epsilon_{\mathrm{n}, \mathrm{rms}} \leq \\
{\left[\epsilon_{\mathrm{sc}}+\epsilon_{\mathrm{rf}}+\epsilon_{\mathrm{mp}}+\epsilon_{\mathrm{B}_{\mathrm{z}}}+\epsilon_{\mathrm{o}}+\epsilon_{\mathrm{T}}\right]}
\end{gathered}
$$

An upper and lower bound on $\epsilon_{\mathrm{n}, \mathrm{rms}}$ can be estimated using Eq. 2.21 [24]. In the following sections we shall elucidate the underlying physics for each of the emittance contributing terms to $\epsilon_{\mathrm{n}, \mathrm{rms}}$. 


\section{Space Charge Emittance, $\epsilon_{\mathrm{sc}}$}

Given the assumption that all the particles in the bunch have the same velocity, in the rest frame of the electron bunch the problem of space charge repulsion can be treated as an electrostatic problem. This repulsive force will manifest itself in beam defocusing; hence, the electron beam emittance will grow. Kim [24] has shown that the space charge emittance term is given by Eq. 2.22,

$$
\epsilon_{\mathrm{i}}^{\mathrm{sc}}=\frac{\pi}{4} \frac{1}{\alpha \mathrm{k}} \frac{1}{\sin \left(\theta_{\mathrm{o}}\right)} \frac{\mathrm{I}_{\mathrm{p}}}{\mathrm{I}_{\mathrm{A}}} \mu_{\mathrm{i}}(\mathrm{AR})
$$

where $\alpha$ is the normalized rf field parameter defined in Eq. 2.23, $\mathrm{k}$ is the wave number

for the structure and equal to $\mathrm{k}=\frac{2 \pi}{\lambda_{\mathrm{rf}}}, \theta_{\mathrm{o}}$ is the laser injection phase with respect to the rf where the zero crossing and the crest of the rf are defined as $\theta_{0}=0^{\circ}$ and $\theta_{\mathrm{o}}=90^{\circ}$ respectively, $\mathrm{I}_{\mathrm{p}}$ is the peak current, $\mathrm{I}_{\mathrm{A}}=17 \mathrm{kA}$ is Alfvén current, and $\mu_{\mathrm{i}}$ is the universal space charge factor, given by Eq. 2.24. $\pi$ is the ratio of the circumference to the diameter of a circle. The normalized rf field parameter is defined by

$$
\alpha=\frac{\mathrm{eE}_{\mathrm{o}}}{2 \mathrm{~m}_{\mathrm{o}} \mathrm{c}^{2} \mathrm{k}}
$$

where $\mathrm{e}$ is the electron charge, $\mathrm{m}_{\mathrm{o}}$ is the rest mass of an electron, $\mathrm{c}$ is the speed of light and $\mathrm{k}$ is the wave number of the structure. $\mathrm{E}_{\mathrm{o}}$ is the peak accelerating field of the rf gun.

$$
\mu_{\mathrm{i}}(\mathrm{AR}) \approx \begin{cases}\frac{1}{3 \mathrm{AR}+5} & \mathrm{i}=\mathrm{x} \\ \frac{1.1}{1+4.5 \mathrm{AR}+2.9 \mathrm{AR}^{2}} & \mathrm{i}=\mathrm{z}\end{cases}
$$

is universal space charge factor for a longitudinal and transverse Gaussian electron beam and it is a function of the aspect ratio, AR, of the electron bunch defined in Eq. 2.25, where $\sigma_{\mathrm{x}}$ and $\sigma_{\mathrm{z}}$ are the transverse and longitudinal rms beam sizes respectively.

$$
\mathrm{AR}=\frac{\sigma_{\mathrm{x}}}{\sigma_{\mathrm{z}}}
$$

The transverse universal space charge term, $\mu_{\mathrm{x}}$, arises for a Gaussian radial distribution from a non-linear lens effect and vanishes for a uniform radial distribution. 
The longitudinal universal space charge term, $\mu_{\mathrm{z}}$, comes from a position (relative to the center of the bunch) dependent lens strength and does not vanish even for a uniform distribution.

\section{rf Emittance, $\epsilon_{\mathrm{rf}}$}

Kim has shown that the emittance contribution due to the accelerating fields in an rf gun results from a defocusing kick at the exit port. In a one and a half cell rf gun, the exit kick of the half cell is cancelled by the input kick of the full cell. In multi-cell guns these cancellation also occurs at each cell-to-cell iris except at the exit port of the rf gun.

The radial component of the force due to the electro-magnetic field in the rf gun is given by Eq. 2.26. It should be pointed out that Kim neglected the term due to the focusing solenoidal, $\mathrm{B}_{\mathrm{z}}$, used in the emittance compensation process.

$$
\mathrm{F}_{\mathrm{r}}=\mathrm{e}\left[\mathrm{E}_{\mathrm{r}}+\left(\beta_{\theta} \mathrm{B}_{\mathrm{z}}-\beta_{\mathrm{z}} \mathrm{B}_{\theta}\right)\right]
$$

where $\mathrm{e}$ is the charge of an electron, $\beta_{\theta}$ and $\beta_{z}$ are the azimuthal and longitudinal components of the normalized electron velocity, and $\mathrm{E}_{\mathrm{r}}$ and $\mathrm{B}_{\theta}$ are components of the time dependent electro-magnetic field in the rf gun.

Eq. 2.27 is the minimum emittance attainable due to $\mathrm{rf}[24]$, where $\alpha$ is the normalized rf field parameter defined in Eq. 2.23, $\mathrm{k}$ is the wave number for the structure, $\sigma_{\mathrm{x}, \mathrm{y}}$ is the transverse rms beam sizes in $\mathrm{mm}$, and $\sigma_{\mathrm{z}}$ is the rms longitudinal bunch length in mrad.

$$
\epsilon_{\mathrm{x}, \mathrm{y}}^{\mathrm{rf}}=\frac{\alpha \mathrm{k}}{\sqrt{2}} \sigma_{\mathrm{x}, \mathrm{y}}^{2} \sigma_{\mathrm{z}}^{2}
$$

\section{Multi-pole Field Asymmetry, $\epsilon_{\mathrm{mp}}$}

In side-coupled structures the maximum accelerating field is not located on the beam line axis. Rather it is offset toward the rf coupling aperture [25]. This offset in $\mathrm{E}_{\mathrm{z}}$ can be thought of as a linear combination of $\mathrm{TM}_{\mathrm{ijk}}$ modes. The $\mathrm{TM}_{110}$ is a dipole 
mode which will cause an emittance growth of the electron beam due to the time dependent transverse kick.

Using the Panofsky Wenzel Theorem Eq. 2.28 [26], we can estimate the emittance growth due to the $\mathrm{TM}_{110}$ component of $\mathrm{E}_{\mathrm{z}}$.

$$
\mathrm{p}_{\mathrm{x}}=\frac{\mathrm{e}}{\mathrm{v}} \int_{0}^{\mathrm{L}}[\mathbf{E}+(\mathbf{v} \times \mathbf{B})]_{\mathrm{x}} \mathrm{dz}
$$

In Eq. 2.28, $\mathrm{p}_{\mathrm{x}}$ is the $\mathrm{x}$ component of momentum vector $\mathbf{p}$, a scalar quantity, e is the electron charge, $\mathbf{v}$ and $\mathbf{v}$ are the constant speed and velocity of an electron, and $\mathbf{E}$ and $\mathbf{B}$ are the electro-magnetic fields in the rf gun. Using the standard definitions, $\mathbf{B} \equiv \nabla \times \mathbf{A}$ and $\mathbf{E} \equiv-\frac{\partial \mathbf{A}}{\partial \mathrm{t}}$, Eq. 2.28 can be rewritten in a form that contains only the vector potential, $\mathbf{A}$, where the Coulomb gauge has been used, since no sources are present.

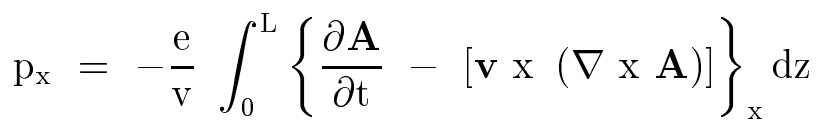

Using the vector identity in Eq. 2.30 we find that the Panofsky Wenzel Theorem can be written in the form of Eq. 2.31, where we have used the definition of the covariant derivative to derive the Panofsky Wenzel Theorem.

$$
\begin{gathered}
\nabla(\mathbf{v} \cdot \mathbf{A})=\mathbf{v} \times(\nabla \times \mathbf{A})+\mathbf{A} \times(\nabla \times \mathbf{v})+(\mathbf{v} \cdot \nabla) \mathbf{A}+(\mathbf{A} \cdot \nabla) \mathbf{v} \\
\mathrm{p}_{\mathrm{x}}=-\frac{\mathrm{e}}{\mathrm{v}} \int_{0}^{\mathrm{L}}\left\{\frac{\mathrm{d} \mathbf{A}}{\mathrm{dt}}-[\nabla(\mathbf{v} \cdot \mathbf{A})]\right\}_{\mathrm{x}} \mathrm{dz}
\end{gathered}
$$

Integrating the covariant derivative and noting that the vector potential is zero outside the cavity, we are left with Eq. 2.32. This result assumes that the velocity vector, $\mathbf{v}$, is a constant in the $\mathrm{z}$ direction.

$$
\mathrm{p}_{\mathrm{x}}=\mathrm{e} \int_{0}^{\mathrm{L}}\left\{\nabla \mathrm{A}_{\mathrm{z}}\right\}_{\mathrm{x}} \mathrm{dz}
$$

Assuming that the $z$ component of the vector potential has the form $A_{z}=A_{o} e^{i \omega t}$, we find that the $\mathrm{TM}_{110}$ dipole mode will induce a time dependent momentum kick for each longitudinal slice of the electron beam. 


$$
\mathrm{p}_{\mathrm{x}}=-\frac{\mathrm{ie}}{\mathrm{c}} \frac{\lambda_{\mathrm{rf}}}{2 \pi} \int_{0}^{\mathrm{L}} \frac{\partial \mathrm{E}_{\mathrm{z}}}{\partial \mathrm{x}} \mathrm{dz}
$$

The transverse divergence of an electron beam is given by $x^{\prime}=\frac{d x}{d t} \frac{d t}{d z}=\frac{v_{x}}{\beta c}$. Therefore the emittance growth of the electron bunch due to the Panofsky Wenzel dipole kick is given by Eq. 2.34. The $\sigma_{z}$ term in Eq. 2.34 is accounted for if we assume that each slice of the electron bunch receives the same transverse time dependent momentum kick. $\left\langle\mathrm{xx}^{\prime}\right\rangle$ due to the dipole kick is zero.. As in Eq. 2.27, $\sigma_{\mathrm{z}}$ has units of mrad.

$$
\epsilon_{\mathrm{mp}}=\beta \gamma \sqrt{\left\langle\mathrm{x}^{2}\right\rangle\left\langle\mathrm{x}^{\prime 2}\right\rangle-\left\langle\mathrm{xx}^{\prime}\right\rangle}=\frac{\mathrm{e}}{\mathrm{m}_{\mathrm{o}} \mathrm{c}^{2}} \frac{\lambda_{\mathrm{rf}}}{2 \pi} \sigma_{\mathrm{x}} \sigma_{\mathrm{z}} \int_{0}^{\mathrm{L}} \frac{\partial \mathrm{E}_{\mathrm{z}}}{\partial \mathrm{x}} \mathrm{dz}
$$

\section{Cathode Magnetic Field Emittance, $\epsilon_{\mathrm{B}_{z}}$}

The conservation of canonical angular momentum will manifest itself in the growth of the electron beam's transverse emittance. The kinetic angular momentum of the electron beam in a field-free region is due to a magnetic field at the cathode of the electron source. The single emittance compensation magnet design causes a finite magnetic field at the cathode which will have the detrimental effect of inducing a small but finite magnetic emittance term to $\epsilon_{\mathrm{n}, \mathrm{rms}}$. It is our goal in this section to derive the emittance term, $\epsilon_{\mathrm{B}_{\mathrm{z}}}$, as a result of the finite magnetic field at the cathode.

The normalized rms transverse beam emittance is defined in Eq. 2.35. The three individual terms of Eq. 2.35 are defined below in Eq. 2.36, 2.37, and 2.38.

$$
\begin{aligned}
& \epsilon_{\mathrm{n}, \mathrm{rms}}=\beta \gamma \sqrt{\left\langle\mathrm{x}^{2}\right\rangle\left\langle\mathrm{x}^{\prime 2}\right\rangle-\left\langle\mathrm{xx}^{\prime}\right\rangle^{2}} \\
& \left\langle\mathrm{x}^{2}\right\rangle=\frac{\iiint \int \mathrm{x}^{2} \rho \mathrm{dx} \mathrm{dx}^{\prime} \mathrm{dy} \mathrm{dy}^{\prime}}{\iiint \int \rho \mathrm{dx} \mathrm{dx}^{\prime} \mathrm{dy} \mathrm{dy}^{\prime}} \\
& \left\langle\mathrm{x}^{\prime 2}\right\rangle=\frac{\iiint \int \mathrm{x}^{\prime 2} \rho \mathrm{dx} \mathrm{dx}^{\prime}{\mathrm{dy} \mathrm{dy}^{\prime}}^{2}}{\iiint \rho \mathrm{dx} \mathrm{dx}^{\prime} \mathrm{dy} \mathrm{dy}^{\prime}} \\
& \left\langle\mathrm{xx}^{\prime}\right\rangle=\frac{\iiint \int \mathrm{xx}^{\prime} \rho \mathrm{dx} \mathrm{dx}^{\prime} \mathrm{dy} \mathrm{dy}^{\prime}}{\iiint \int \rho \mathrm{dx} \mathrm{dx}^{\prime} \mathrm{dy}_{\mathrm{dy}}}
\end{aligned}
$$


Assume the KV distribution [27], given by Eq. 2.39,

$$
\rho=\delta\left(\frac{\mathrm{x}^{2}+\mathrm{y}^{2}}{\mathrm{a}^{2}}+\frac{\mathrm{x}^{\prime 2}+\mathrm{y}^{\prime 2}}{\mathrm{~b}^{2}}-1\right)
$$

where the maximum radius of the laser spot on the cathode is given by $a=R_{o}$ and $b$ is the maximum divergence of the beam off the cathode defined as given by Busch's Theorem [28]. It is equal to $b=\frac{e R_{o}}{2 \beta \gamma m_{o} c} B_{z}$. The KV distribution can be visualized as a uniformly filled 3-D hyper-ellipsoidal shell in 4-D trace space. The KV distribution has the property that the charge density across the beam is constant and the forces associated with the self-fields vary linearly with radius

We first calculate the normalizing term in Eq. 2.36, 2.37, and 2.38, given by Eq 2.40.

$$
\iiint \int \delta\left(\frac{\mathrm{r}^{2}}{\mathrm{a}^{2}}+\frac{\mathrm{r}^{\prime 2}}{\mathrm{~b}^{2}}-1\right) \mathrm{dx} \mathrm{dx}^{\prime} \mathrm{dy} \mathrm{dy}^{\prime}=\pi^{2} \mathrm{a}^{2} \mathrm{~b}^{2}
$$

Next we calculate the numerator of Eq. 2.36, which is the beam size term, with the result presented in Eq. 2.41.

$$
\iiint \int \mathrm{x}^{2} \delta\left(\frac{\mathrm{r}^{2}}{\mathrm{a}^{2}}+\frac{\mathrm{r}^{\prime 2}}{\mathrm{~b}^{2}}-1\right) \mathrm{dx} d \mathrm{x}^{\prime} \mathrm{dy} \mathrm{dy}^{\prime}=\frac{\pi^{2}}{4} \mathrm{a}^{4} \mathrm{~b}^{2}
$$

This result combined with the normalizing term gives the following result: The mean square beam size is equal to the hard edge beam radius squared divided by four,

$$
\left\langle\mathrm{x}^{2}\right\rangle=\frac{\mathrm{a}^{2}}{4}
$$

The numerator of Eq. 2.37 is given by Eq. 2.43

$$
\iiint \int \mathrm{x}^{\prime 2} \delta\left(\frac{\mathrm{r}^{2}}{\mathrm{a}^{2}}+\frac{\mathrm{r}^{2}}{\mathrm{~b}^{2}}-1\right) \mathrm{dx} d \mathrm{x}^{\prime} \mathrm{dy} \mathrm{dy}^{\prime}=\frac{\pi^{2}}{4} \mathrm{a}^{2} \mathrm{~b}^{4}
$$

Therefore, the mean square value of the transverse beam divergence is given by Eq. 2.44.

$$
\left\langle\mathrm{x}^{\prime 2}\right\rangle=\frac{\mathrm{b}^{2}}{4}
$$


The numerator of the correlation term, Eq. 2.38, is given by Eq. 2.45

$$
\left.\iiint \int \mathrm{xx}^{\prime} \delta\left(\frac{\mathrm{r}^{2}}{\mathrm{a}^{2}}+\frac{\mathrm{r}^{2}}{\mathrm{~b}^{2}}-1\right)\right) \mathrm{dx} \mathrm{dx}^{\prime} \mathrm{dy} \mathrm{dy}^{\prime}=0
$$

The correlation term $\left\langle\mathrm{xx}^{\prime}\right\rangle$ is clearly zero since the angular integration after a change of variables is an odd function over symmetric limits. Inserting the theoretically calculated values of the beam size, beam divergence, and correlation into the definition of the normalized rms emittance given by Eq. 2.35, we find that the emittance growth due to the finite longitudinal magnetic field at the cathode is given by Eq. 2.46.

$$
\epsilon_{\mathrm{n}, \mathrm{rms}}=\beta \gamma \sqrt{\left\langle\mathrm{x}^{2}\right\rangle\left\langle\mathrm{x}^{\prime 2}\right\rangle-\left\langle\mathrm{xx}^{\prime}\right\rangle^{2}}=\frac{\beta \gamma}{4} \mathrm{ab}
$$

The KV distribution has the property that the charge density across the beam is constant and the forces associated with the self-fields vary linearly with radius

All that remains is to insert the values of the $a$ and $b$ terms. Therefore the normalized emittance due to the finite magnetic field at the cathode is

$$
\epsilon_{\mathrm{B}_{\mathrm{z}}}=\frac{\mathrm{e}}{8 \mathrm{~m}_{\mathrm{o}} \mathrm{c}} \mathrm{R}_{\mathrm{o}}^{2} \mathrm{~B}_{\mathrm{z}}
$$

At the Brookhaven Accelerator Test Facility (ATF), the laser spot size on the cathode is $1 \mathrm{~mm}$ radius. Inserting this value into Eq. 2.47, we find that the magnetic emittance term scales as $\epsilon_{\mathrm{B}_{\mathrm{z}}}=0.01 \frac{\pi \mathrm{mm} \mathrm{mrad}}{\mathrm{G}}$. This is negligible in the emittance budget of NGP design.

\section{Thermal Emittance, $\epsilon_{\mathrm{o}}$}

The intrinsic emittance of a photocathode is determined by the difference in laser photon energy and the work function of the cathode material where the work function of the cathode material has been lowered by the application of a large accelerating field gradient on the cathode. The lowering of the work function by the application of an electric field gradient is called the Schottky Effect [29]. The Schottky Effect increases the initial kinetic energy available to the photoemitted electrons over the 
energy provided to the photoemitted electrons by the actual physical temperature of the photoemitting material.

In this section we will calculate an upper limit to the intrinsic emittance of a copper photocathode, including the Schottky Effect. The intrinsic emittance is called the thermal emittance, $\epsilon_{0}$, but should not be confused with the actual temperature of the cathode material, which will be discussed in the next subsection.

We will calculate the normalized rms transverse beam emittance as defined by Eq. 2.48. For completeness the three individual terms of Eq. 2.48 are defined in Eqs. 2.49, 2.50, and 2.51.

$$
\begin{gathered}
\epsilon_{\mathrm{n}, \mathrm{rms}}=\beta \gamma \sqrt{\left\langle\mathrm{x}^{2}\right\rangle\left\langle\mathrm{x}^{\prime 2}\right\rangle-\left\langle\mathrm{xx}^{\prime}\right\rangle^{2}} \\
\left\langle\mathrm{x}^{2}\right\rangle=\frac{\iint \mathrm{x}^{2} \rho \mathrm{dxd \textrm {x } ^ { \prime }}}{\iint \rho \mathrm{dxd \textrm {x } ^ { \prime }}} \\
\left\langle\mathrm{x}^{\prime 2}\right\rangle=\frac{\iint \mathrm{x}^{\prime 2} \rho \mathrm{dxdx^{ \prime }}}{\iint \rho \mathrm{dxd \textrm {x } ^ { \prime }}} \\
\left\langle\mathrm{xx}^{\prime}\right\rangle=\frac{\iint \mathrm{xx}^{\prime} \rho \mathrm{dx} \mathrm{dx}}{\iint \rho \mathrm{dx} \mathrm{dx}^{\prime}}
\end{gathered}
$$

The $\left\langle\mathrm{x}^{2}\right\rangle$ term of Eq. 2.49 is given in Eq. 2.52. Where $\mathrm{R}_{\mathrm{o}}$ is the radius of the laser spot on the cathode.

$$
\left\langle\mathrm{x}^{2}\right\rangle=\frac{\iint \mathrm{r}^{3} \cos ^{2}(\theta) \rho \mathrm{dr} \mathrm{d} \theta}{\iint \rho \mathrm{r} \mathrm{dr} \mathrm{d} \theta}=\frac{\mathrm{R}_{\mathrm{o}}^{2}}{4}
$$

The $\left\langle\mathrm{x}^{2}\right\rangle$ term manifests itself from the energy difference between the laser photon energy and the Schottky-corrected work function give in Eq. 2.53.

$$
\delta \mathrm{E}=\mathrm{E}_{\gamma}-\phi_{\text {eff }}=\mathrm{E}_{\gamma}-\left[\phi_{\mathrm{o}}-\sqrt{\beta_{\gamma} \mathrm{E}_{\mathrm{o}} \sin \left(\theta_{\mathrm{o}}\right)}\right]
$$

where $\mathrm{E}_{\gamma}$ is the laser photon energy, $\phi_{\mathrm{eff}}$ is the effective work function of the photocathode, $\phi_{\mathrm{o}}$ is the zero field work function of the photocathode, $\beta_{\gamma}$ is the field enhancement factor of the photoemitting surface, $E_{o}$ is the peak accelerating field in the rf gun, and $\theta_{\mathrm{o}}$ is the laser injection phase with respect to the rf. 
In this calculation we will assume that all of the excess energy goes into the transverse velocity of the electrons. In fact, the angular velocity distribution of photoemitted electrons as a function of the applied accelerating field is not well understood. We have also assumed that $\mathrm{v}_{\mathrm{x}}^{2}$ is a constant.

$$
\left\langle\mathrm{x}^{\prime 2}\right\rangle=\frac{\iint \mathrm{x}^{\prime 2} \rho \mathrm{dx} \mathrm{dx}^{\prime}}{\iint \rho \mathrm{dx} \mathrm{dx}^{\prime}}=\left(\frac{1}{\beta \mathrm{c}}\right)^{2} \mathrm{v}_{\mathrm{x}}^{2}=\left(\frac{1}{\beta \mathrm{c}}\right)^{2}\left(\frac{2 \delta \mathrm{E}}{\mathrm{m}_{\mathrm{o}}}\right)
$$

Where $\delta \mathrm{E}$ is the energy difference between the laser photon energy and the effective work function of the material, given in Eq. 2.53, $\mathrm{m}_{\mathrm{o}}$ is the rest mass of an electron, $\mathrm{c}$ is the speed of light, and $\beta$ is the normalized velocity defined in Eq. 2.2.

$$
\left\langle\mathrm{x}^{2}\right\rangle=\frac{1}{\beta^{2}}\left(\frac{2 \delta \mathrm{E}}{\mathrm{m}_{\mathrm{o}} \mathrm{c}^{2}}\right)
$$

We assume the correlation term $\left\langle\mathrm{xx}^{\prime}\right\rangle$ is zero since the position of the photoemission process does not effect the magnitude of the transverse velocity.

Inserting the theoretically calculated values of the beam size, beam divergence, and the correlation terms into the definition of the normalized rms emittance, we find that the intrinsic emittance of a photocathode is given by Eq. 2.56. This result assumes that the electron's longitudinal velocity at photoemission is small compared to the velocity of light, $\gamma=1$.

$$
\epsilon_{\mathrm{o}}=\beta \gamma \sqrt{\left\langle\mathrm{x}^{2}\right\rangle\left\langle\mathrm{x}^{\prime 2}\right\rangle-\left\langle\mathrm{xx}^{\prime}\right\rangle^{2}}=\frac{\mathrm{R}_{\mathrm{o}}}{2} \sqrt{\frac{2 \delta \mathrm{E}}{\mathrm{m}_{\mathrm{o}} \mathrm{c}^{2}}}
$$

We are now in a position to estimate the upper limit of the thermal emittance of an electron beam produced by a copper photocathode in a time-dependent electric field environment. The modification of the zero field work function by the application of an accelerating gradient will suppress the material's zero field work function by an amount given by Eq. 2.53.

Fig. 2.3 represents the upper limit of the intrinsic emittance of a copper photocathode as a function of applied field gradient where we have used the parameters in Table 2.1 which are identical to our experimental beam dynamics studies conducted at the Brookhaven Accelerator Test Facility (ATF). In this calculation we have assumed that the energy difference between the laser photon energy and the Schottky 


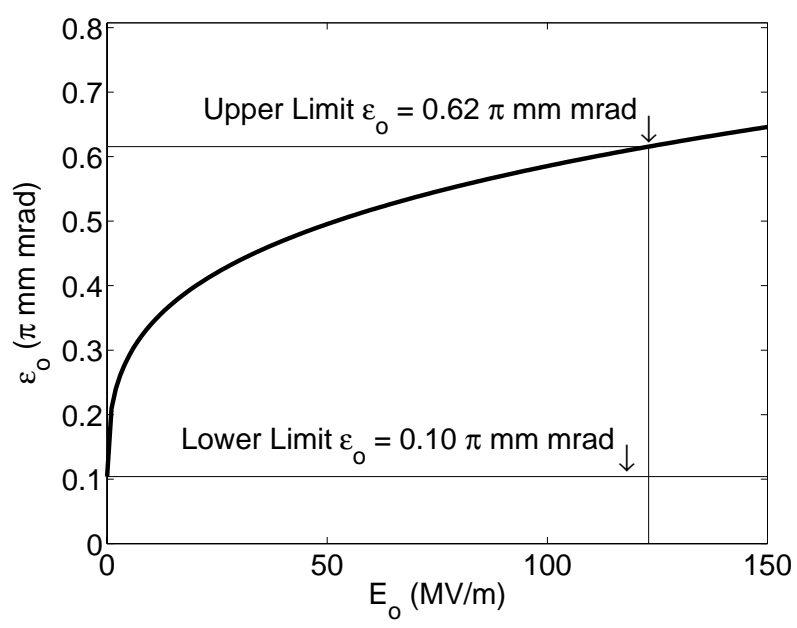

Figure 2.3: Thermal emittance contribution as a function of applied accelerating field gradient.

\begin{tabular}{|c|c|}
\hline Spot Size edge-to-edge & $2 \mathrm{~mm}$ \\
\hline Cathode Field Level & $123 \frac{\mathrm{MV}}{\mathrm{m}}$ \\
\hline Laser Injection Phase $\left(\theta_{\mathrm{o}}\right)$ & $53^{\mathrm{O}}$ \\
\hline$\beta_{\gamma}$ & 1 \\
\hline $\mathrm{E}_{\gamma}=266 \mathrm{~nm}$ & $4.66 \mathrm{eV}$ \\
\hline $\mathrm{Cu}$ Work Function, $\phi_{\mathrm{o}}$ & $4.65 \mathrm{eV}$ \\
\hline
\end{tabular}

Table 2.1: Cathode spot size and rf parameters. Where the $\theta_{o}$ is defined from the zero crossing of the rf.

modified work function goes into the transverse direction.

The normal operational parameters presented in Table 2.1 are used for the beam dynamics experiments present in this dissertation. The upper limit of the thermal emittance of the electron beam is calculated to be $\epsilon_{\mathrm{o}}=0.62 \pi \mathrm{mm} \mathrm{mrad}$.

For the theoretical development of the thermal emittance, $\epsilon_{\mathrm{o}}$, contribution to $\epsilon_{\mathrm{n}, \mathrm{rms}}$ we have assume that the actual temperature of the cathode does not effect the photoemission process [30]. In Fig. 2.4 we represent the energy spectrum of the photoemitted electrons of a free electron gas which obeys the Fermi-Dirac distribution [31], given in Eq. 2.57 , for $0^{\circ} \mathrm{K}$ and $300^{\circ} \mathrm{K}$. 


$$
\mathrm{f}\left(\epsilon_{\mathrm{f}}\right)=\frac{1}{\mathrm{e}^{\frac{\left(\epsilon_{\mathrm{f}}-\mu_{\mathrm{cp}}\right)}{k_{\mathrm{B}} \mathrm{T}}}+1}
$$

where $\epsilon_{\mathrm{f}}$ is the Fermi energy, $\mu_{\mathrm{cp}}$ is the chemical potential, $\mathrm{k}_{\mathrm{B}}$ is the Boltzmann's constant, and $\mathrm{T}$ is the materials temperature in degrees Kelvin.

The hard edge energy cutoff at $0^{\circ} \mathrm{K}$ is modified for the $300^{\circ} \mathrm{K}$ case in a region on the order of $\mathrm{k}_{\mathrm{B}} \mathrm{T}=26 \mathrm{meV}$. This is a $3 \%$ effect when compared to $\delta \mathrm{E}=390 \mathrm{meV}$. We have assumed that the chemical potential of the cathode material is temperature independent. This is a reasonable assumption since the chemical potential up to $300^{\circ} \mathrm{K}$ only changes by $.01 \%$. Since $\epsilon_{\mathrm{o}}$ scales as the square root of $\delta \mathrm{E}$, the temperature dependence of the photoemission process is not considered significant.

It should be pointed out that $\epsilon_{\mathrm{o}}$ can be reduced by multiple electron-phonon scattering events in the crystalline lattice of the photo-emitting material before the electrons are emitted from the cathode.

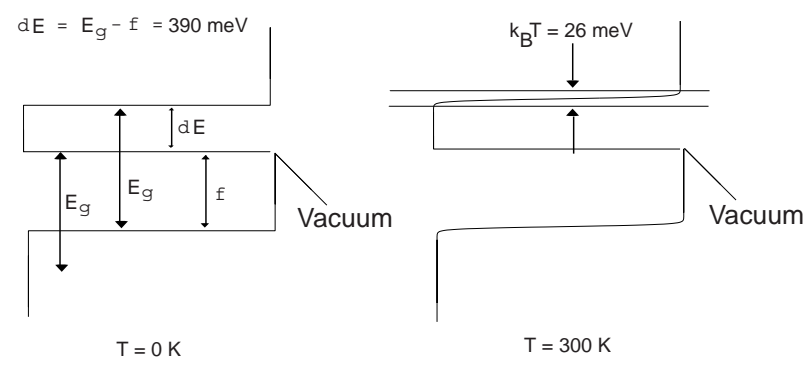

Figure 2.4: The energy spectrum of photoemitted electrons for $0^{\circ} \mathrm{K}$ and $300^{\circ} \mathrm{K}$.

\section{Physical Temperature, $\epsilon_{\mathrm{T}}$}

The physical temperature of the cathode will provide an additional emittance contribution, $\epsilon_{\mathrm{T}}$. The actual physical temperature of the 1.6 cell $\mathrm{rf}$ gun during normal operation is $55^{\circ} \mathrm{C}$. Using a modified form of Eq. 2.56, where $\delta \mathrm{E}$ now equals the total energy allowed in one dimension of real space by the equipartition theorem from statistical mechanics, which is equal to $\delta \mathrm{E}=\frac{\mathrm{k}_{\mathrm{B}} \mathrm{T}}{2}$, we find that the actual physical temperature of the photoemitting surface will contribute $\epsilon_{\mathrm{T}}=0.12 \pi \mathrm{mm} \mathrm{mrad}$ to the total emittance budget. Thus, 


\begin{tabular}{|c|c|c|}
\hline$\epsilon_{\mathrm{sc}}$ & Space Charge & 0.00 \\
\hline$\epsilon_{\mathrm{rf}}$ & Radio Frequency & 0.20 \\
\hline$\epsilon_{\mathrm{mp}}$ & Multi-Pole Field & 0.15 \\
\hline$\epsilon_{\mathrm{B}_{\mathrm{z}}}$ & Cathode Magnetic Field & 0.04 \\
\hline$\epsilon_{\mathrm{o}}$ & Thermal & 0.62 \\
\hline$\epsilon_{\mathrm{T}}$ & Physical Temperature & 0.12 \\
\hline
\end{tabular}

Table 2.2: Estimation of the individual emittance terms in the zero charge limit, in units of $\pi$ mm mrad.

$$
\epsilon_{\mathrm{o}}=\frac{\mathrm{R}_{\mathrm{o}}}{2} \sqrt{\frac{\mathrm{k}_{\mathrm{B}} \mathrm{T}}{\mathrm{m}_{\mathrm{o}} \mathrm{c}^{2}}}=0.12 \pi \mathrm{mm} \operatorname{mrad}
$$

where $R_{o}$ is the radius of the laser spot on the cathode, $k_{B}$ is Boltzmann's constant, $\mathrm{T}$ is the temperature of the cathode in degrees Kelvin, $\mathrm{m}_{\mathrm{o}}$ is the rest mass of an electron, and $\mathrm{c}$ is the speed of light.

\subsubsection{Emittance Estimation in the Zero Charge Limit}

We are now in a position to estimate the normalized rms emittance of a zero charge electron beam. This result will be compared to our experimental results in Section 5.6.2 of the normalized rms emittance as a function of charge in the limit of zero electron bunch charge. Therefore, we shall assume that the emittance contribution from space charge is zero. In this limit there will be no transverse beam size growth; consequently we shall use the cathode spot size of $2 \mathrm{~mm}$ diameter for the calculation of the rf and multi-pole field emittance terms. The cathode magnetic field term is for residual field of $4 \mathrm{G}$ which was the value for our experimental beam dynamics studies. The thermal term is for a copper photocathode with an accelerating gradient of $123 \frac{\mathrm{MV}}{\mathrm{m}}$ and a laser injection phase of $53^{\circ}$ from the zero crossing. The normal operating temperature of the gun is $55^{\circ} \mathrm{C}$.

In Table 2.2 we present the calculated values of the individual emittance contribution terms from Section 2.3.3 to the total normalized rms emittance. Adding these individual emittances in both linear and quadratic fashion, Eq. 2.21, we find that in the limit of zero charge the theoretically predicated normalized rms emittance will be 
between the limits presented in Eq. 2.59.

$$
0.68 \pi \mathrm{mm} \mathrm{mrad} \leq \epsilon_{\mathrm{n}, \mathrm{rms}} \leq 1.13 \pi \mathrm{mm} \operatorname{mrad}
$$

In Section 5.6.2 we find that the least square fit to the experimental data indicates that in the limit of zero charge the normalized rms emittance of the electron beam is $0.8 \pi \mathrm{mm}$ mrad.

The comparison of the theoretically predicated value of the normalized rms emittance in the limit of zero charge with our experimental results from Section 5.6.2 indicates that our upper limit value of the thermal emittance term, $\epsilon_{\mathrm{o}}=0.62 \pi \mathrm{mm} \mathrm{mrad}$, is correct.

\subsection{Simulations}

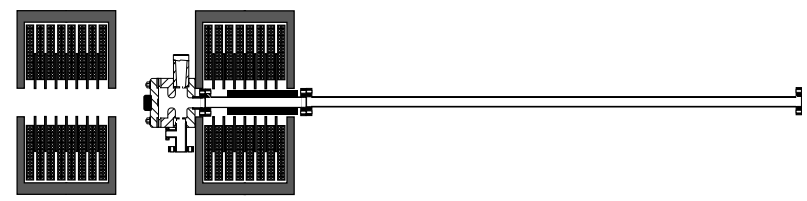

Figure 2.5: Low energy beam line used in the PARMELA simulations of this section.

In this section we shall present the results of our PARMELA [3] simulations of the model beam line shown in Fig. 2.5. The model beam line consists of the 1.6 cell rf gun and the emittance compensation magnet followed by a 5 meter long drift space. Both flat-top and Gaussian longitudinal laser pulse shapes, shown in Fig. 2.6, were simulated. The PARMELA [3] input parameters for each set of simulations will be presented in each section. Table 2.3 defines the symbols used in the simulation parameter list. The thermal emittance is assumed to be identically zero. To arrive at a more precise value of $\epsilon_{\mathrm{n}, \mathrm{rms}}$, the reader should add in quadrature the calculated thermal emittance contribution, $\epsilon_{\mathrm{O}}[32]$.

Unless otherwise noted, the quoted emittance is the minimum emittance that PARMELA [3] has calculated at any position along the beam line, from the exit of the gun to the end of the drift section. 


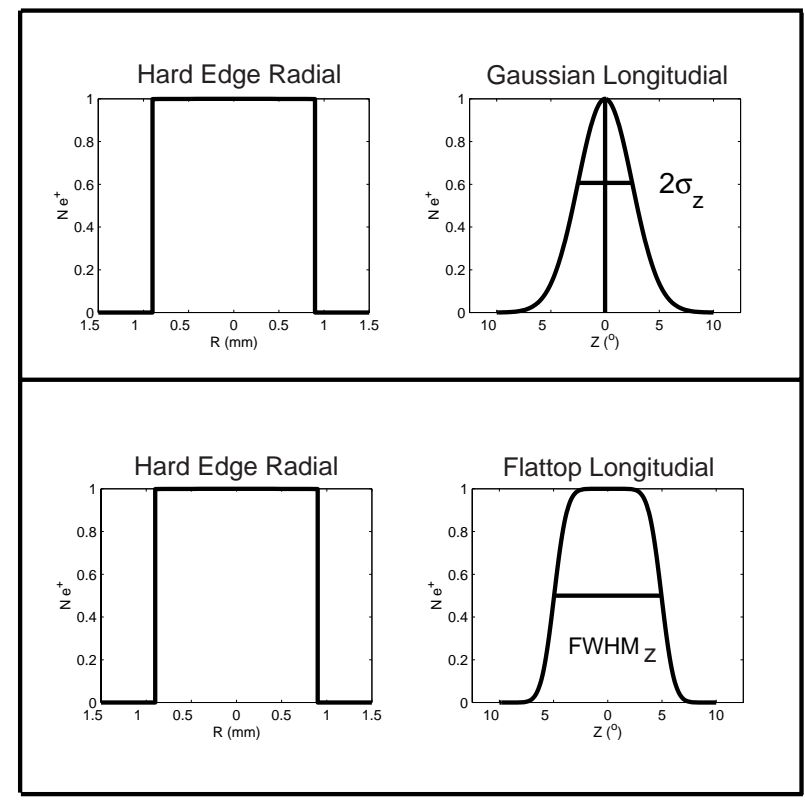

Figure 2.6: Transverse and longitudinal laser profile used in the following PARMELA simulations.

\begin{tabular}{|l|l|}
\hline Number of Macro-Particles & $\mathrm{N}_{\mathrm{e}^{-}}$ \\
\hline Thermal Emittance & $\epsilon_{\mathrm{o}}$ \\
\hline Initial Kinetic Energy & $\mathrm{KE}_{\mathrm{o}}$ \\
\hline Peak Accelerating Field & $\mathrm{E}_{\mathrm{o}}$ \\
\hline Field Balance & $\mathrm{E}_{2} / \mathrm{E}_{1}$ \\
\hline Total Charge & $\mathrm{Q}_{\mathrm{t}}$ \\
\hline Cathode Spot Size & $\mathrm{R}_{\mathrm{o}}$ \\
\hline Longitudinal Flat-Top & $\mathrm{FWHM}_{\mathrm{z}}$ \\
\hline Longitudinal Gaussian & $\sigma_{\mathrm{z}}$ \\
\hline PARMELA Injection Phase & $\Theta_{\mathrm{o}}$ \\
\hline Laser Injection Phase & $\theta_{\mathrm{o}}$ \\
\hline Peak Magnetic Field & $\mathrm{B}_{\mathrm{z}}$ \\
\hline
\end{tabular}

Table 2.3: Definition of symbols used throughout Section 2.4. 


\begin{tabular}{|l|l|}
\hline $\mathrm{N}_{\mathrm{e}^{-}}$ & $10^{4}$ \\
\hline$\epsilon_{\mathrm{o}}$ & $0.00 \pi \mathrm{mm} \mathrm{mrad}$ \\
\hline $\mathrm{KE}_{\mathrm{o}}$ & $0.5 \mathrm{eV}$ \\
\hline $\mathrm{E}_{\mathrm{o}}$ & VARY \\
\hline $\mathrm{E}_{2} / \mathrm{E}_{1}$ & 1.00 \\
\hline $\mathrm{Q}_{\mathrm{t}}$ & $1 \mathrm{nC}$ \\
\hline $\mathrm{R}_{\mathrm{o}}$ & $0.90 \mathrm{~mm}$ \\
\hline $\mathrm{FWHM}_{\mathrm{z}}$ & $10 \mathrm{psec}$ \\
\hline$\theta_{\mathrm{o}}$ & VARY \\
\hline $\mathrm{B}_{\mathrm{z}}$ & $2.71 \mathrm{kG}$ \\
\hline
\end{tabular}

Table 2.4: PARMELA simulation parameters for $\gamma$ versus $\theta_{\mathrm{o}}$ and $\mathrm{E}_{\mathrm{o}}$, using a $10 \mathrm{psec}$ $\mathrm{FWHM}_{\mathrm{z}}$ flat-top longitudinal laser profile. Note that the solenoidal field is constant for all values of $E_{o}$ and $\theta_{0}$.

These sets of low energy emittance compensation simulations allow us to estimate the performance of a high energy emittance compensated photoinjector without the increased complexity of the post-acceleration process. The underlying hypothesis of these simulations is that we can attain the performance of the low energy injector while freezing in the transverse emittance at high energy by varying the beam line parameters.

\subsubsection{Electron Beam Energy, $\gamma m_{o} c^{2}$, Versus Laser Injection Phase, $\theta_{0}$, and Accelerating Field Gradient, $\mathrm{E}_{\mathrm{o}}$}

Electron beam energy, $\gamma \mathrm{m}_{\mathrm{o}} \mathrm{c}^{2}$, is an important beam parameter since all emittance compensated photoinjectors depend on the $\frac{1}{\gamma^{2}}$ effect to mitigate the deleterious effect of space charge. Fig. 2.7 represents PARMELA [3] simulations of the 1.6 cell rf gun electron beam energy as a function of field gradient and laser injection phase where $90^{\circ}$ is the rf crest and $0^{\circ}$ is the zero crossing. Parameters for these simulations are given in Table 2.4.

Since the 1.6 cell rf gun has a nonstandard half cell length, it is not possible to use Kim's theory [24] to analytically model the electron beam energy at the end of the gun. Serafini [33] has calculated the electron beam energy for the case of half cell 


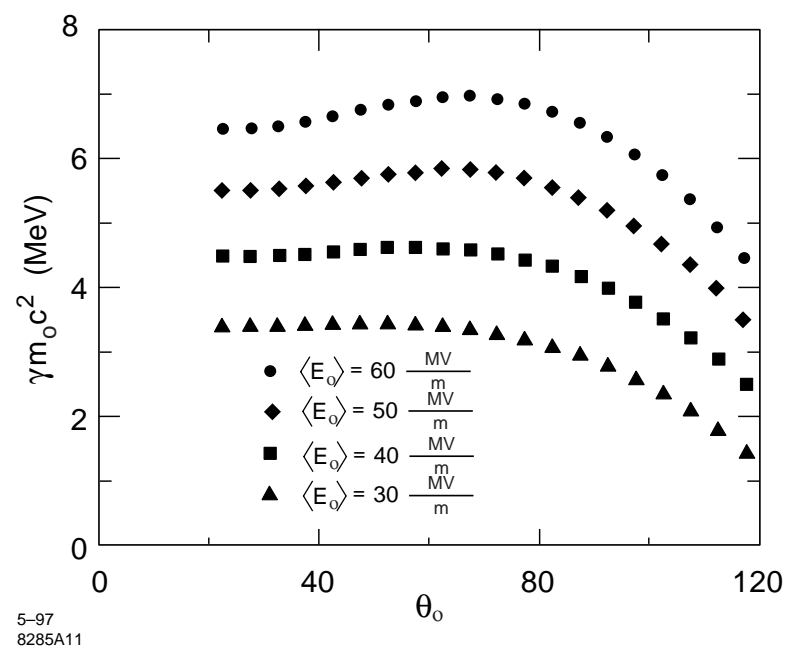

Figure 2.7: PARMELA simulations of the electron beam energy versus laser injection phase and field gradients.

length perturbation, $d$. In this case the cell-to-cell iris is located $z_{1}=(1+d) \frac{\lambda}{4}$ from the cathode plane and $\mathrm{z}_{2}=\mathrm{z}_{1}+\frac{\lambda}{2}$ is the location of the beam exit port. Eq. 2.60 and 2.61 are the beam energy at the end of the half cell and at the exit of the full cell, respectively.

$$
\begin{aligned}
& \gamma_{1}=1+\frac{\pi \alpha}{2}\left[1+\frac{5}{4} \mathrm{~d}-\left(\frac{5}{16}+\frac{\pi^{2}}{24}\right) \mathrm{d}^{2}\right] \\
& \gamma_{2}=1+\frac{3 \pi \alpha}{2}\left[1+\frac{5}{12} \mathrm{~d}-\left(\frac{5}{2}+\frac{\pi^{2}}{3}\right) \frac{\mathrm{d}^{2}}{24}\right]
\end{aligned}
$$

The 1.6 cell $\mathrm{rf}$ gun has the perturbation value $\mathrm{d}=0.25$ and $\alpha$ is the normalized accelerating field gradient which was defined in Eq. 2.23. Comparing these analytical results to our PARMELA [3] simulations in Table 2.5 we find that the agreement is within $2.5 \%$, in the normal operating range of $E_{0}$, which is between $100-150 \frac{\mathrm{MV}}{\mathrm{m}}$.

\subsection{2 $\epsilon_{\mathrm{n}, \mathrm{rms}}$ Versus $\mathrm{E}_{\mathrm{o}}$ and $\mathrm{B}_{\mathrm{z}}$}

As we discussed in Section 2.3.3, the effect of space charge on $\epsilon_{\mathrm{n}, \mathrm{rms}}$ is minimized with increasing field gradient but the emittance growth due to the rf defocusing kick at the exit of the full cell increases as the peak accelerating gradient is increased. These 


\begin{tabular}{|l|l|l|l|}
\hline $\mathrm{E}_{\mathrm{o}}\left(\frac{\mathrm{MV}}{\mathrm{m}}\right)$ & Analytical & PARMELA & \% Error \\
\hline 146.9 & 6.810 & 6.952 & 2.04 \\
\hline 122.4 & 5.760 & 5.782 & 0.38 \\
\hline 97.95 & 4.711 & 4.605 & 2.30 \\
\hline 73.47 & 3.662 & 3.419 & 7.11 \\
\hline
\end{tabular}

Table 2.5: Comparison of Serafini's analytical results to PARMELA simulations of the maximum energy out of the NGP as a function of $E_{0}$.

\begin{tabular}{|l|l|}
\hline $\mathrm{N}_{\mathrm{e}^{-}}$ & $10^{4}$ \\
\hline$\epsilon_{\mathrm{o}}$ & $0.00 \pi \mathrm{mm} \mathrm{mrad}$ \\
\hline $\mathrm{KE}_{\mathrm{o}}$ & $0.5 \mathrm{eV}$ \\
\hline $\mathrm{E}_{\mathrm{o}}$ & VARY \\
\hline $\mathrm{E}_{2} / \mathrm{E}_{1}$ & 1.00 \\
\hline $\mathrm{Q}_{\mathrm{t}}$ & $1 \mathrm{nC}$ \\
\hline $\mathrm{R}_{\mathrm{o}}$ & $0.90 \mathrm{~mm}$ \\
\hline $\mathrm{FWHM}_{\mathrm{z}}$ & $10 \mathrm{psec}$ \\
\hline$\Theta_{\mathrm{o}}$ & $-154^{\circ}$ \\
\hline $\mathrm{B}_{\mathrm{z}}$ & VARY \\
\hline
\end{tabular}

Table 2.6: PARMELA simulation parameters for $\epsilon_{\mathrm{n}, \mathrm{rms}}$ versus $\mathrm{E}_{\mathrm{o}}$ and $\mathrm{B}_{\mathrm{z}}$. 
two effects scale inversely with each other, which implies that there is an optimum operating field gradient. Fig. 2.8 bears this hypothesis out. In these simulations we have found the optimum magnetic field for a given accelerating gradient and also the optimum accelerating field gradient in the emittance compensated domain.

Due to space charge compensation, the $\epsilon_{\mathrm{n}, \mathrm{rms}}$ decreases as a function of field gradient; up to a peak field of $\mathrm{E}_{\mathrm{o}}=143 \frac{\mathrm{MV}}{\mathrm{m}}$. Field gradients above this limit cause the $\epsilon_{\mathrm{n}, \mathrm{rms}}$ to start to increase due to rf emittance, as seen in Fig. 2.8. Parameters for these simulations are given in Table 2.6.

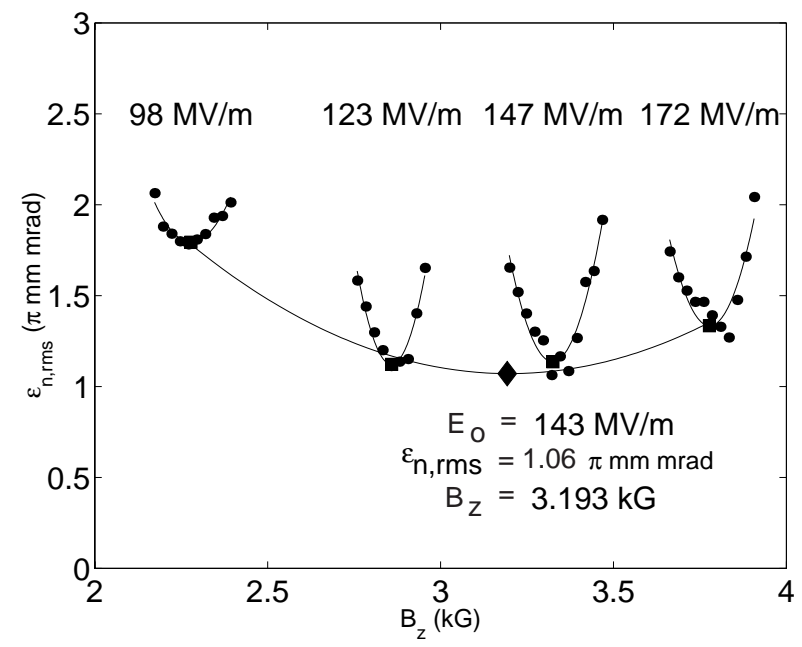

Figure 2.8: PARMELA simulation results for $\epsilon_{\mathrm{n}, \mathrm{rms}}$ versus $\mathrm{E}_{\mathrm{o}}$ and $\mathrm{B}_{\mathrm{z}}$.

The final result of these sets of simulations is that the optimum field gradient is $\mathrm{E}_{\mathrm{o}}=143 \frac{\mathrm{MV}}{\mathrm{m}}$ where we can expect to achieve a normalized emittance of $\epsilon_{\mathrm{n}, \mathrm{rms}}=1.06 \pi \mathrm{mm}$ mrad with a magnetic field strength of $\mathrm{B}_{\mathrm{z}}=3.193 \mathrm{kG}$. These PARMELA [3] simulations determined the physics requirements on the single emittance compensation magnet. The POISSON simulations and the design of the single emittance compensation solenoidal magnet are presented in Chapter 4.

\subsection{3 $\epsilon_{\mathrm{n}, \mathrm{rms}}$ Versus $\frac{\mathrm{E}_{2}}{\mathrm{E}_{1}}$ and $\mathrm{B}_{\mathrm{z}}$}

The dependence of the electron beams transverse normalized rms emittance, $\epsilon_{\mathrm{n}, \mathrm{rms}}$, is studied as a function of the field balance, FB, and peak magnetic field, $B_{z}$, in the 


\begin{tabular}{|l|l|}
\hline $\mathrm{N}_{\mathrm{e}^{-}}$ & $10^{4}$ \\
\hline$\epsilon_{\mathrm{o}}$ & $0.00 \pi \mathrm{mm} \mathrm{mrad}$ \\
\hline $\mathrm{KE}_{\mathrm{o}}$ & $0.5 \mathrm{eV}$ \\
\hline $\mathrm{E}_{\mathrm{o}}$ & $147 \frac{\mathrm{MV}}{\mathrm{m}}$ \\
\hline $\mathrm{E}_{2} / \mathrm{E}_{1}$ & VARY \\
\hline $\mathrm{Q}_{\mathrm{t}}$ & $1 \mathrm{nC}$ \\
\hline $\mathrm{R}_{\mathrm{o}}$ & $0.90 \mathrm{~mm}$ \\
\hline $\mathrm{FWHM}_{\mathrm{z}}$ & $10 \mathrm{psec}$ \\
\hline$\Theta_{\mathrm{o}}$ & $-150^{\circ}$ \\
\hline $\mathrm{B}_{\mathrm{z}}$ & VARY \\
\hline
\end{tabular}

Table 2.7: PARMELA simulation parameters for $\epsilon_{\mathrm{n}, \mathrm{rms}}$ versus $\frac{\mathrm{E}_{2}}{\mathrm{E}_{1}}$.

1.6 cell $\mathrm{rf}$ gun. In Fig. 3.12 the field balance is shown to be a function of the mode separation between $f_{0}$ and $f_{\pi}$. In the region of minimum mode separation the field balance can vary $\pm 25 \%$ due to experimental error. As in previous simulations, we use electro-magnetic field maps from SUPERFISH [5] for input into PARMELA [3]. Fig. 2.9 represents the magnitude of the longitudinal accelerating field imbalance mapped into PARMELA [3]. Parameters for these simulations are given in Table 2.7.

The simulated beam line consists of a 1.6 cell S-Band rf gun with a bucked pair of emittance compensation magnets. A 5 meter long drift is located at the output of the gun to allow for the emittance compensation process to evolve numerically. The beam line is shown in Fig. 2.5.

Fig. 2.10 represents the results of our PARMELA [3] simulations for the three different field balances discussed previously. In the field balance range that was simulated, $\pm 25 \%$, there is no significant difference in the minimum emittance attainable. There is a difference in solenoidal magnet settings used to attain the same minimum emittance. As expected, the solenoidal field variation correlates with the amplitude of the divergent kick due to the exit port of the rf gun. 


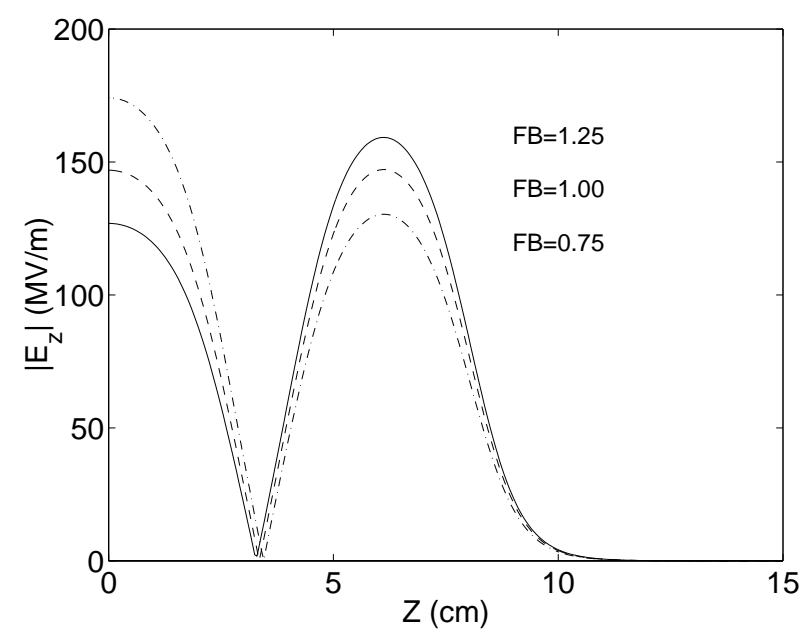

Figure 2.9: SUPERFISH field maps used in PARMELA to investigate the dependence of $\epsilon_{\mathrm{n}, \mathrm{rms}}$ on field balance.

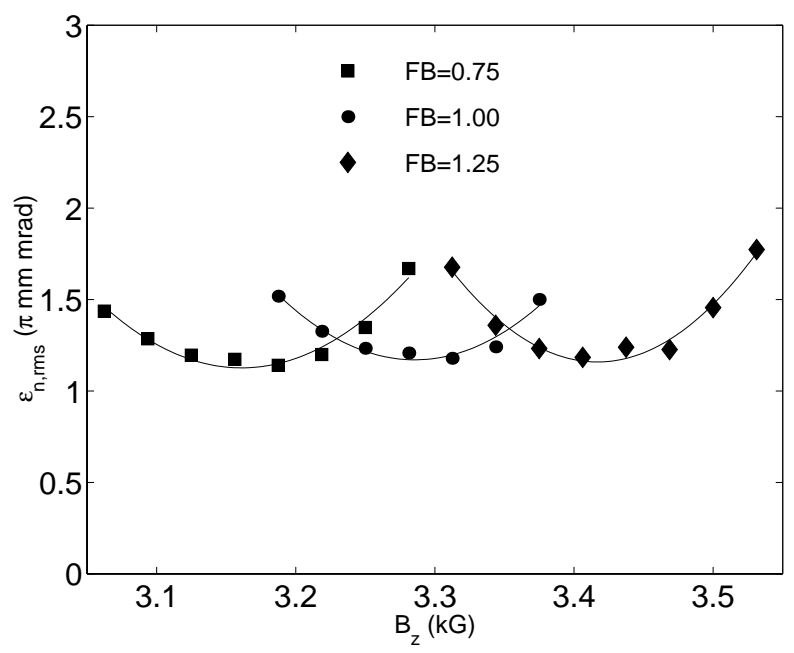

Figure 2.10: PARMELA simulations of $\epsilon_{\mathrm{n}, \mathrm{rms}}$ versus $\frac{\mathrm{E}_{2}}{\mathrm{E}_{1}}$. 


\begin{tabular}{|l|l|}
\hline $\mathrm{N}_{\mathrm{e}^{-}}$ & $10^{4}$ \\
\hline$\epsilon_{\mathrm{o}}$ & $0.00 \pi \mathrm{mm} \mathrm{mrad}$ \\
\hline $\mathrm{KE}_{\mathrm{o}}$ & $0.5 \mathrm{eV}$ \\
\hline $\mathrm{E}_{\mathrm{o}}$ & $147 \frac{\mathrm{MV}}{\mathrm{m}}$ \\
\hline $\mathrm{E}_{2} / \mathrm{E}_{1}$ & 1.00 \\
\hline $\mathrm{Q}_{\mathrm{t}}$ & $1 \mathrm{nC}$ \\
\hline $\mathrm{R}_{\mathrm{o}}$ & $0.90 \mathrm{~mm}$ \\
\hline $\mathrm{FWHM}_{\mathrm{z}}$ & $10 \mathrm{psec}$ \\
\hline$\Theta_{\mathrm{o}}$ & $-150^{\circ}$ \\
\hline $\mathrm{B}_{\mathrm{z}}$ & VARY \\
\hline
\end{tabular}

Table 2.8: PARMELA simulation parameters for $\epsilon_{\mathrm{n}, \mathrm{rms}}$ versus $\mathrm{B}_{z}$.

\subsection{4 $\epsilon_{\mathrm{n}, \mathrm{rms}}$ Versus $\mathrm{B}_{\mathrm{z}}$}

We have used a longitudinal flat-top electron bunch profile with a full width half maximum $\left(\mathrm{FWHM}_{\mathrm{z}}\right)$ of 10 psec shown in Fig. 2.6 to study $\epsilon_{\mathrm{n}, \mathrm{rms}}$ versus $\mathrm{B}_{\mathrm{z}}$. Parameters for these simulations are given in Table 2.8. Fig. 2.11 represents the minimum emittance that can be attained at a position along the beam line for a given setting of the emittance compensation magnet. Two features stand out in Fig. 2.11. Feature A in Fig. 2.11 is a local minimum with $\epsilon_{\mathrm{n}, \mathrm{rms}}=1.57 \pi \mathrm{mm} \mathrm{mrad}$ at a peak magnetic field strength of $B_{z}=3.093 \mathrm{kG}$. Feature $B$ in Fig. 2.11 is the global minimum. It is located at $\epsilon_{\mathrm{n}, \mathrm{rms}}=1.17 \pi \mathrm{mm}$ mrad with a peak magnetic field strength of $\mathrm{B}_{\mathrm{z}}=3.286 \mathrm{kG}$.

The local minimum (A) is due to the head and tail of the electron bunch being slightly out of alignment with the core, in transverse phase space. The global minimum (B) occurs when the head, core, and tail align with the least possible projected area. This effect is demonstrated in Fig. 2.12. We have also demonstrated this effect using the program SLICER. This program allows us to eliminate different slices of the electron bunch in the calculation of $\epsilon_{\mathrm{n}, \mathrm{rms}}$ without affecting the beam dynamics downstream of a physical aperture that is commonly used to eliminate the beam halo. SLICER is capable of both longitudinal and radial slicing. However, since the beam halo is due to the head and tail of the bunch, the halo is eliminated with longitudinal slicing alone; therefore. Therefore the radial slicing capability of SLICER was not 


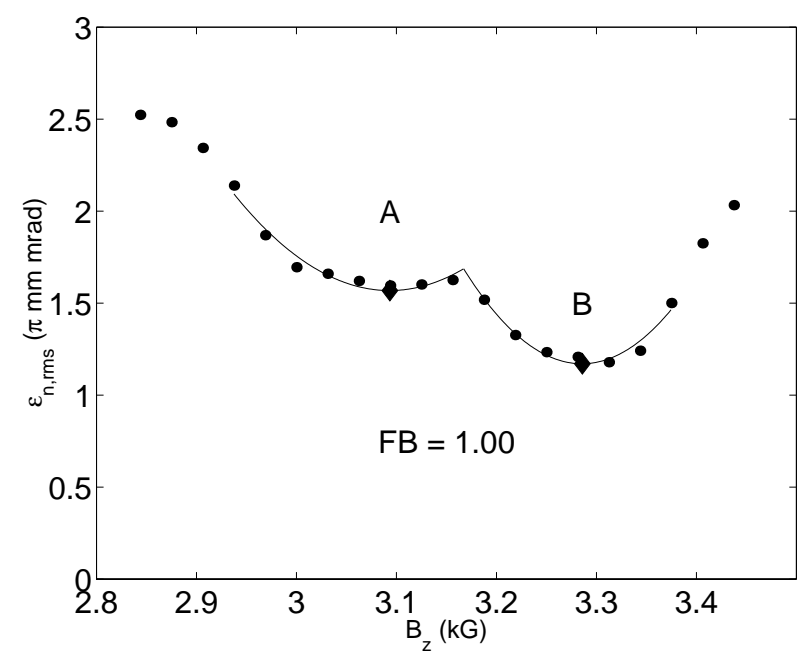

Figure 2.11: PARMELA simulation results for emittance versus peak magnet field. Note that cusp is a artifact of the separates fits used in region A and B.

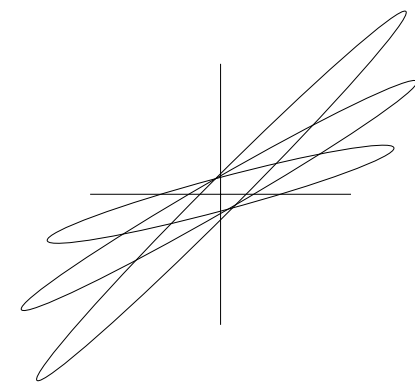

A

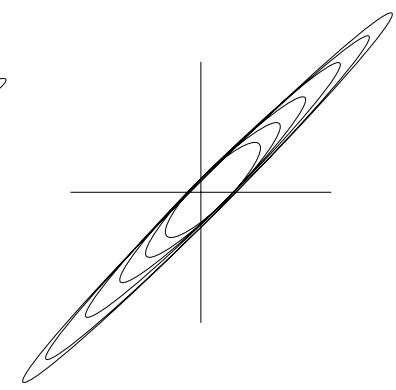

B

Figure 2.12: Diagram of the emittance compensation process. 


\begin{tabular}{|l|l|}
\hline $\mathrm{N}_{\mathrm{e}^{-}}$ & $10^{4}$ \\
\hline$\epsilon_{\mathrm{o}}$ & $0.00 \pi \mathrm{mm} \mathrm{mrad}$ \\
\hline $\mathrm{KE}_{\mathrm{o}}$ & $0.5 \mathrm{eV}$ \\
\hline $\mathrm{E}_{\mathrm{o}}$ & $147 \frac{\mathrm{MV}}{\mathrm{m}}$ \\
\hline $\mathrm{E}_{2} / \mathrm{E}_{1}$ & 1.00 \\
\hline $\mathrm{Q}_{\mathrm{t}}$ & $1 \mathrm{nC}$ \\
\hline $\mathrm{R}_{\mathrm{o}}$ & $0.90 \mathrm{~mm}$ \\
\hline $\mathrm{FWHM}$ & $10 \mathrm{psec}$ \\
\hline$\theta_{\mathrm{o}}$ & VARY \\
\hline $\mathrm{B}_{\mathrm{z}}$ & $3.312 \mathrm{kG}$ \\
\hline
\end{tabular}

Table 2.9: PARMELA simulation parameters for $\epsilon_{\mathrm{n}, \mathrm{rms}}$ versus $\theta_{\mathrm{o}}$.

needed to eliminate the beam halo.

\subsection{5 $\epsilon_{\mathrm{n}, \mathrm{rms}}$ Versus $\theta_{\mathrm{O}}$}

The simulations in this section are for a 10 psec longitudinal flat-top laser pulse with a constant solenoidal field setting close to the optimum magnetic field value found in Section 2.4.4. Parameters for these simulations are given in Table 2.9. This represents the method of least descent, in which a minimum of the emittance due to a given beam line parameter is found and another parameter is varied while the rest of the beam line parameters are kept constant. This does not allow for the study of the correlation between different beam parameters. We will investigate these correlations in Section 2.4.7 where we compare the beam line parameter space for both flat-top and Gaussian longitudinal laser pulses using 2-D parameter scans of PARMELA [3].

In these simulations the total charge of the electron bunch is constant. This has real physical significance since changing $\theta_{0}$, the Schottky Effect would decrease the effective electron yield of the photoemitting material. Consequently, to maintain a constant charge in the electron bunch over a wide range of $\theta_{0}$, excess laser energy must be available to experimentally realize these simulation results.

Fig. 2.13 indicates that with a laser injection phase of $\theta_{0}=59.7^{\circ}$ it is possible to attain a transverse normalized rms emittance of $\epsilon_{\mathrm{n}, \mathrm{rms}}=1.26 \pi \mathrm{mm}$ mrad with a longitudinal flat-top laser pulse. The maximum energy occurs at a laser injection 


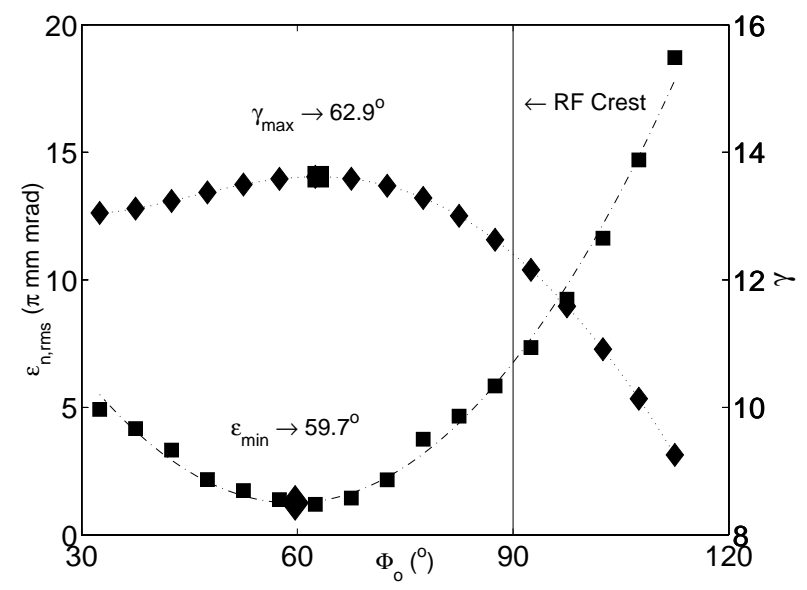

Figure 2.13: PARMELA simulations of $\epsilon_{\mathrm{n}, \mathrm{rms}}$ and $\gamma$ versus laser injection phase $\theta_{\mathrm{o}}$ for a longitudinal flat-top laser profile.

phase of $\theta_{\mathrm{o}}=62.9^{\circ}$ with $\gamma=13.62$. Comparing these results we see that the optimum laser injection phase is not the phase that will maximize the electron energy. Rather it is closer to the zero crossing by $3.2^{\circ}$.

\subsection{6 $\epsilon_{\mathrm{n}, \mathrm{rms}}$ Versus Total Charge, $\mathrm{Q}_{\mathrm{t}}$}

The beam line simulated is the 1.6 cell rf gun and emittance compensation magnet with a long drift, as shown in Fig. 2.5. There is no post-acceleration in these simulations. The solenoid field strength is not varied but kept constant. Fig. 2.14 represents the beam's normalized rms emittance at a z position of $165 \mathrm{~cm}$ from the cathode plane. Parameters for these simulations are given in Table 2.10.

Three operating regions are clearly present in Fig 2.14 .

- Region $\mathrm{A}$ is the uncompensated rf gun region where the transverse space charge forces are not sufficient to overcome the solenoidal focusing of the compensation magnet.

- Region B is the linear space charge emittance compensation region where the transverse space charge forces are of sufficient strength to be compensated by the solenoidal magnet. When there is a match between the space charge defocusing and solenoidal focusing we approach the emittance minimum. 


\begin{tabular}{|l|l|}
\hline $\mathrm{N}_{\mathrm{e}^{-}}$ & $10^{4}$ \\
\hline$\epsilon_{\mathrm{o}}$ & $0.00 \pi \mathrm{mm} \mathrm{mrad}$ \\
\hline $\mathrm{KE}_{\mathrm{o}}$ & $0.5 \mathrm{eV}$ \\
\hline $\mathrm{E}_{\mathrm{o}}$ & $147 \frac{\mathrm{MV}}{\mathrm{m}}$ \\
\hline $\mathrm{E}_{2} / \mathrm{E}_{1}$ & 1.00 \\
\hline $\mathrm{Q}_{\mathrm{t}}$ & VARY \\
\hline $\mathrm{R}_{\mathrm{o}}$ & $0.90 \mathrm{~mm}$ \\
\hline $\mathrm{FWHM}_{\mathrm{z}}$ & $10 \mathrm{psec}$ \\
\hline$\Theta_{\mathrm{o}}$ & $-150^{\circ}$ \\
\hline $\mathrm{B}_{\mathrm{z}}$ & $3.312 \mathrm{kG}$ \\
\hline
\end{tabular}

Table 2.10: PARMELA simulation parameters for $\epsilon_{\mathrm{n}, \mathrm{rms}}$ versus Total Charge, $\mathrm{Q}_{\mathrm{t}}$.

- Region $\mathrm{C}$ is where the transverse space charge forces are greater than the solenoidal focusing and we have emittance growth.

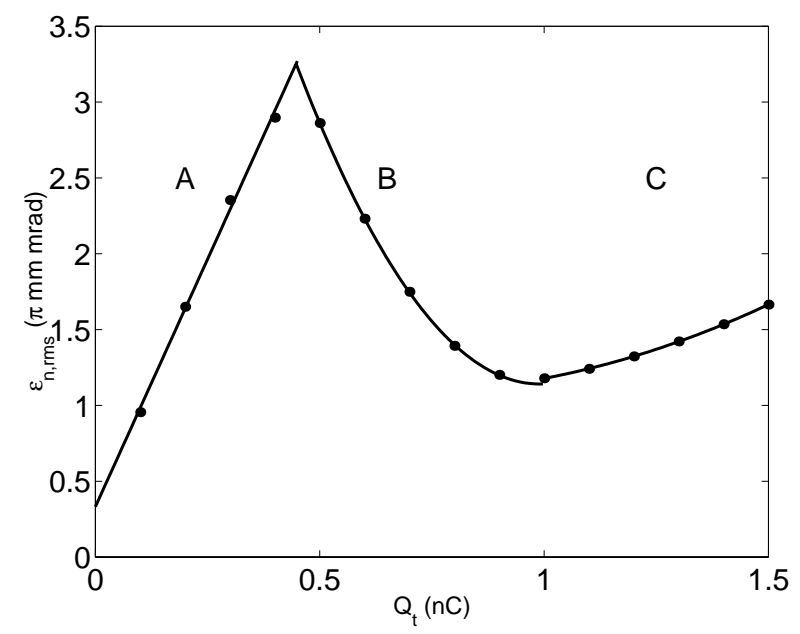

Figure 2.14: PARMELA simulations of $\epsilon_{\mathrm{n}, \mathrm{rms}}$ versus $\mathrm{Q}_{\mathrm{t}}$ at $\mathrm{z}=165 \mathrm{~cm}$ from the cathode plane.

It should be noted that the zero charge intercept represents the rf contribution to the emittance at zero charge. The two different y intercepts for the two different charge regions is due to the different beam sizes that exist in the gun in the different charge regions. More charge means a larger radius, which implies that more of the rf field is sampled by the beam; therefore, the contribution to the rf emittance will be 


\begin{tabular}{|l|l|}
\hline $\mathrm{N}_{\mathrm{e}^{-}}$ & $10^{4}$ \\
\hline$\epsilon_{\mathrm{o}}$ & $0.00 \pi \mathrm{mm} \mathrm{mrad}$ \\
\hline $\mathrm{KE}_{\mathrm{o}}$ & $0.5 \mathrm{eV}$ \\
\hline $\mathrm{E}_{\mathrm{o}}$ & $147 \frac{\mathrm{MV}}{\mathrm{m}}$ \\
\hline $\mathrm{E}_{2} / \mathrm{E}_{1}$ & 1.00 \\
\hline $\mathrm{Q}_{\mathrm{t}}$ & $1 \mathrm{nC}$ \\
\hline $\mathrm{R}_{\mathrm{o}}$ & VARY \\
\hline $\mathrm{FWHM}_{\mathrm{z}}$ & $10 \mathrm{psec}$ \\
\hline$\sigma_{\mathrm{z}}$ & $2.5 \mathrm{psec}$ \\
\hline$\theta_{\mathrm{o}}$ & VARY \\
\hline $\mathrm{B}_{\mathrm{z}}$ & VARY \\
\hline
\end{tabular}

Table 2.11: PARMELA simulation parameters for $\epsilon_{\mathrm{n}, \mathrm{rms}}$ versus Pulse Shape.

larger.

\subsection{7 $\epsilon_{\mathrm{n}, \mathrm{rms}}$ Versus Pulse Shape}

In the past, the method of least descent was used to find the optimum operational parameters. This does not allow for the study of correlations between beam parameters. The use of 2-D parameter scans represents the first step in fully mapping the n-dimensional phase space in which space charge dominated emittance compensated photoinjectors operate. Parameters used in these simulations are given in Table 2.11.

In the previous section of this chapter we simulated a 10 psec longitudinal flattop laser profile. In this section we extend these simulations to study the emittance compensation process of a $\sigma_{\mathrm{z}}=2.5$ psec Gaussian longitudinal laser profiles. Also, we remove the constraint of using the method of least descent. To accomplish this, we have conducted emittance scans using PARMELA [3] as in the previous section, but now we produce a $2 \times 2$ matrix array of $\epsilon_{\mathrm{n}, \mathrm{rms}}$ values. These 2-D parameter scans, using PARMELA [3], were conducted for $\epsilon_{\mathrm{n}, \mathrm{rms}}$ versus $\mathrm{B}_{\mathrm{z}}, \theta_{\mathrm{o}}$ and $\epsilon_{\mathrm{n}, \mathrm{rms}}$ versus $\mathrm{B}_{\mathrm{z}}, \mathrm{R}_{\mathrm{o}}$ for both Gaussian and flat-top longitudinal laser profiles.

In the 10 psec $\mathrm{FWHM}_{\mathrm{z}}$ longitudinal flat-top case, we scanned $\epsilon_{\mathrm{n}, \mathrm{rms}}$ versus $\mathrm{B}_{\mathrm{z}}$ and $\theta_{\mathrm{o}}$ for a $0.9 \mathrm{~mm}$ radius $1 \mathrm{nC}$ electron beam. This data was fit to a bi-cubic function using MATLAB to produce Fig. 2.15. Using the fitted data, the optimum laser injection 


\section{$\mathrm{FWHM}_{\mathrm{z}}=10 \mathrm{psec}$}
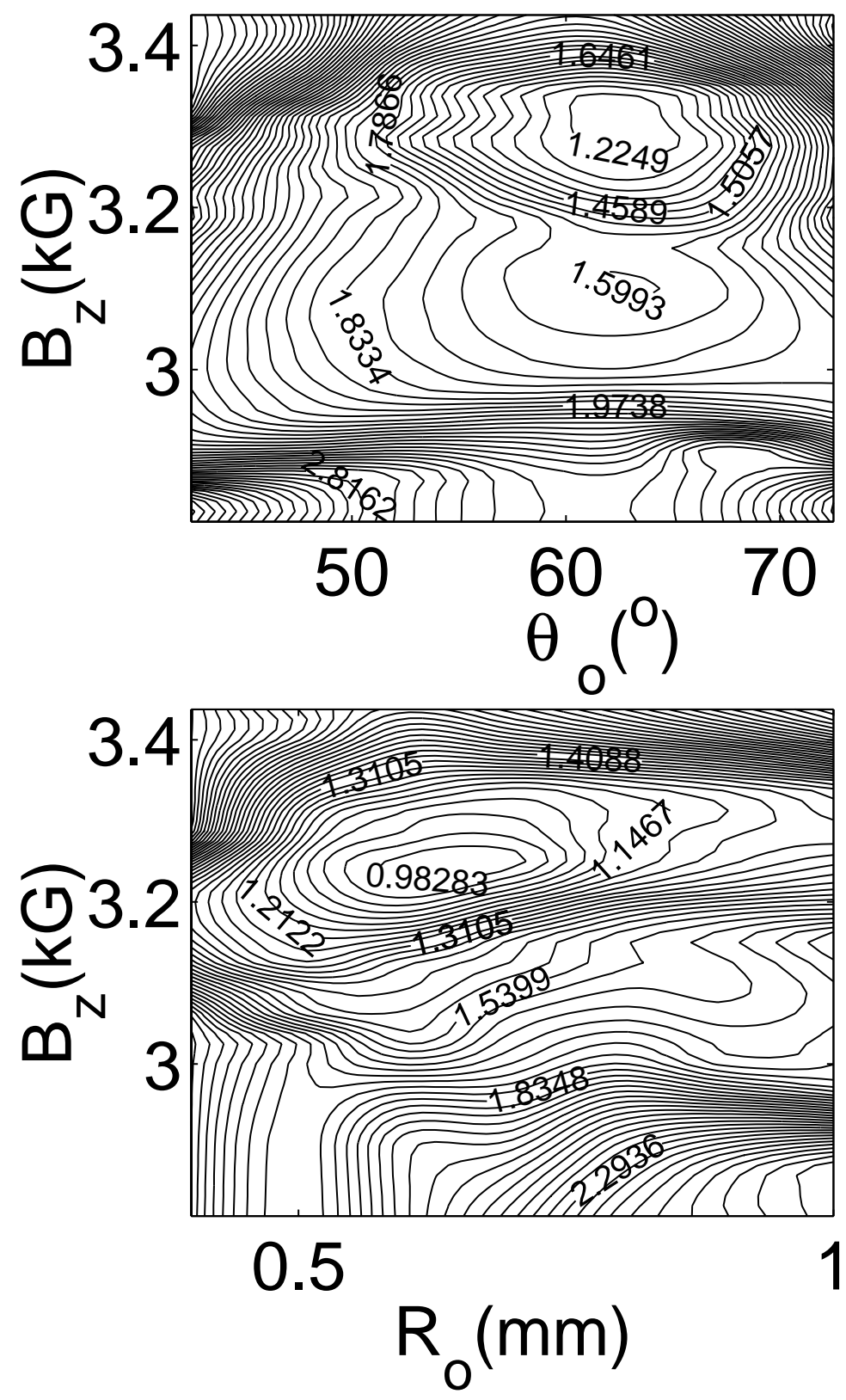

Figure 2.15: 2-D parameter scans using PARMELA for a 10 psec $\mathrm{FWHM}_{\mathrm{z}}$ flat-top longitudinal laser pulse, $\epsilon_{\mathrm{n}, \mathrm{rms}}$ versus cathode spot size, $\mathrm{R}_{\mathrm{o}}$, laser injection phase, $\theta_{\mathrm{o}}$, and magnetic field, $\mathrm{B}_{\mathrm{z}}$. 

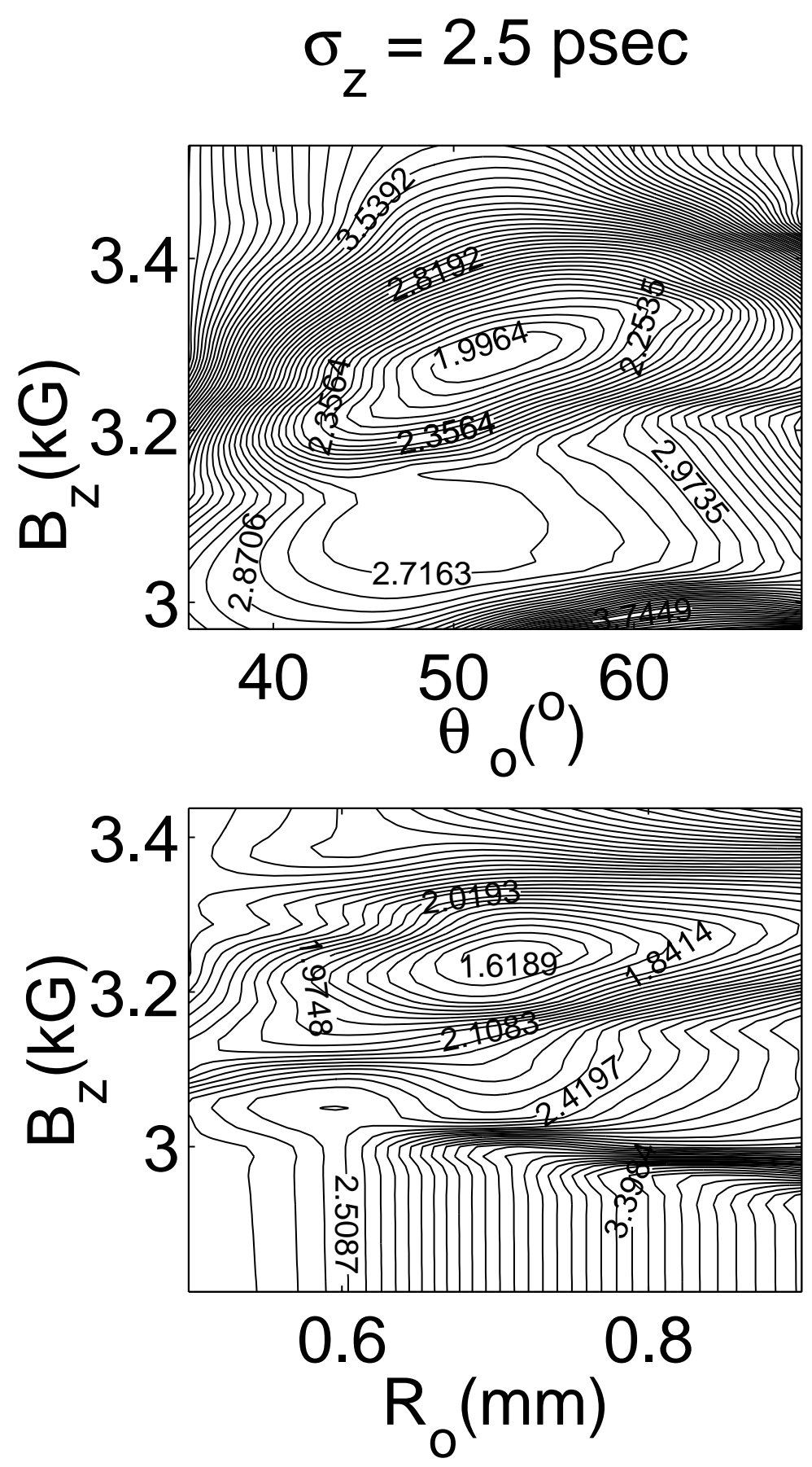

Figure 2.16: 2-D parameter scans using PARMELA for a $\sigma_{\mathrm{z}}=2.5$ psec Gaussian longitudinal laser pulse, $\epsilon_{\mathrm{n}, \mathrm{rms}}$ versus cathode spot size, $\mathrm{R}_{\mathrm{o}}$, laser injection phase, $\theta_{\mathrm{o}}$, and magnetic field, $\mathrm{B}_{\mathrm{z}}$. 


\begin{tabular}{|c|c|c|}
\hline & Gaussian $\sigma_{\mathrm{z}}=2.5 \mathrm{psec}$ & Flat-top $\mathrm{FWHM}_{\mathrm{z}}=10 \mathrm{psec}$ \\
\hline \hline$\epsilon_{\mathrm{n}, \mathrm{rms}}(\pi \mathrm{mm} \mathrm{mrad})$ & 1.95 & 1.18 \\
\hline $\mathrm{B}_{\mathrm{z}}(\mathrm{kG})$ & 3.284 & 3.312 \\
\hline$\theta_{\mathrm{o}}\left({ }^{\circ}\right)$ & 51.6 & 62.3 \\
\hline \hline$\epsilon_{\mathrm{n}, \mathrm{rms}}(\pi \mathrm{mm} \mathrm{mrad})$ & 1.57 & 0.95 \\
\hline $\mathrm{B}_{\mathrm{z}}(\mathrm{kG})$ & 3.250 & 3.259 \\
\hline $\mathrm{R}_{\mathrm{o}}(\mathrm{mm})$ & 0.71 & 0.65 \\
\hline
\end{tabular}

Table 2.12: 2-D PARMELA results for both Gaussian and flat-top longitudinal laser profiles, $\epsilon_{\mathrm{n}, \mathrm{rms}}$ versus $\mathrm{B}_{\mathrm{z}}$ and $\theta_{\mathrm{o}}$ and $\epsilon_{\mathrm{n}, \mathrm{rms}}$ versus $\mathrm{B}_{\mathrm{z}}$ and $\mathrm{R}_{\mathrm{o}}$.

phase was found and used in the simulations of $\epsilon_{n, r m s}$ versus $B_{z}$ and $R_{0}$. This second PARMELA [3] scan is also found in Fig. 2.15.

The same process was used to study the $\sigma_{\mathrm{z}}=2.5$ psec Gaussian case. Fig. 2.16 represents the $\epsilon_{\mathrm{n}, \mathrm{rms}}$ versus $\mathrm{B}_{\mathrm{z}}$ and $\theta_{\mathrm{o}}$ for a $0.9 \mathrm{~mm}$ radius $1 \mathrm{nC}$ electron beam. Using the fitted data, the optimum laser injection phase was found and used in the simulations of $\epsilon_{\mathrm{n}, \mathrm{rms}}$ versus $\mathrm{B}_{\mathrm{z}}$ and $\mathrm{R}_{\mathrm{o}}$. This fourth PARMELA [3] scan is found in Fig. 2.16.

Table 2.12 represents the optimum operational beam parameters for PARMELA [3] simulations that consist of a BNL/SLAC/UCLA 1.6 cell S-Band emittance compensated photoinjector with an identical bucking magnet and 5 meter drift. There is no post-acceleration to high energy which would allow for the freezing of the low emittance that is possible at the gun test facility.

\subsection{Conclusions}

We have estimated the emittance contribution terms in the limit of zero charge and find that our theoretically predicated value of the normalized rms emittance, $\epsilon_{\mathrm{n}, \mathrm{rms}}$, is given by Eq. 2.62.

$$
0.68 \pi \mathrm{mm} \mathrm{mrad} \leq \epsilon_{\mathrm{n}, \mathrm{rms}} \leq 1.13 \pi \mathrm{mm} \operatorname{mrad}
$$


The comparison between simulation, theory, and the experiment data from Chapter 5 is excellent. Therefore, we believe that our theoretical prediction of the dominate emittance term $\epsilon_{\mathrm{o}}=0.62 \pi \mathrm{mm} \mathrm{mrad}$ is correct.

We have conducted PARMELA [3] simulations of the model beam line shown in Fig. 2.5 using field maps of SUPERFISH [5] and POISSON. These simulations use the method of least descent to find the optimum operating point of the model beam line. PARMELA [3] simulations of $\gamma$ versus $\theta_{0}$ and $E_{o}$ were conducted. These simulations agree with theoretical predictions. Our simulations of $\epsilon_{\mathrm{n}, \mathrm{rms}}$ versus $\mathrm{E}_{\mathrm{o}}$ and $\mathrm{B}_{\mathrm{z}}$ indicate that the optimum accelerating field gradient to minimize the combination of $\epsilon_{\mathrm{sc}}$ and $\epsilon_{\mathrm{rf}}$ is $143 \frac{\mathrm{MV}}{\mathrm{m}}$. Simulations of $\epsilon_{\mathrm{n}, \mathrm{rms}}$ versus $\frac{\mathrm{E}_{2}}{\mathrm{E}_{1}}$ show no significant emittance growth, using field maps from SUPERFISH [5] that are unbalanced $\pm 25 \%$. Two features in Fig. 2.11, which is a plot of $\epsilon_{\mathrm{n}, \mathrm{rms}}$ versus $\mathrm{B}_{\mathrm{z}}$, stand out. These are the local and global minimum of $\epsilon_{\mathrm{n}, \mathrm{rms}}$. Simulations of $\epsilon_{\mathrm{n}, \mathrm{rms}}$ versus $\theta_{\mathrm{o}}$ show that the laser injection phase for maximum energy and minimum emittance are not equal. The minimum emittance injection phase is closer to the zero crossing of the rf. This is due to the rf compression that reduces $\sigma_{\mathrm{z}}$ and corresponding $\epsilon_{\mathrm{rf}}$, which scales as $\sigma_{\mathrm{z}}^{2}$. Three regimes in Fig. 2.14, which is the plot of $\epsilon_{\mathrm{n}, \mathrm{rms}}$ versus $\mathrm{Q}_{\mathrm{t}}$, stand out. There are the uncompensated rf gun region, the linear space charge emittance compensation region, and the emittance growth region.

We have extended the method of least descent used in the previous PARMELA [3] simulations into 2-D parameter scans that allow for the study of correlation between different simulation parameters. For a Gaussian laser profile of $\sigma_{\mathrm{z}}=2.5$ psec with a $0.71 \mathrm{~mm}$ radius laser spot size and $3.250 \mathrm{kG}$ compensation magnet field, we find that optimum performance is $\epsilon_{\mathrm{n}, \mathrm{rms}}=1.57 \pi \mathrm{mm} \mathrm{mrad}$. We have found that the optimum performance attained, using a 10 psec flat-top laser pulse with a 0.65 mm radius laser spot on the cathode and $3.259 \mathrm{kG}$ compensation magnet field, is $\epsilon_{\mathrm{n}, \mathrm{rms}}=0.95 \pi \mathrm{mm}$ mrad. The simulation results of both Gaussian and flat-top 2-D parameter scans using PARMELA [3] were presented in Table 2.12.

PARMELA [3] simulations show that by using a 10 psec longitudinal flat-top laser pulse a $50 \%$ improvement in emittance will be gained over that of a Gaussian laser pulse with a $\sigma_{\mathrm{z}}$ of 2.5 psec. 
Chapter 3

\section{Microwave Theory and Measurements}




\subsection{Introduction}

In this chapter we will present the necessary microwave theory and low power rf measurements for both the low power rf gun brass test model and oxygen free high conductive (OFHC) grade II copper $(\mathrm{Cu}) 1.6$ cell S-band rf gun. The rf gun design, manufacturing techniques, and cleaning issues are also discussed. Low power $\mathrm{rf}$ testing results include longitudinal, transverse, and $\Theta$ dependent longitudinal accelerating field measurements. Waveguide to $\pi$-mode coupling measurements will also be presented using the Smith Chart representation.

\subsection{Microwave Measurement Theory}

In this section we will discuss the necessary microwave theory to understand the low power rf measurements and rf tuning that was conducted on the 1.6 cell rf gun. Resonant frequency, field balance, and both rf and cell-to-cell coupling will need to be determined.

\subsubsection{Scattering Parameters}

The scattering matrix [34] is defined in Eq. 3.1.

$$
\left[\begin{array}{c}
\mathrm{V}_{1}^{-} \\
\mathrm{V}_{2}^{-} \\
\vdots \\
\mathrm{V}_{\mathrm{N}}^{-}
\end{array}\right]=\left[\begin{array}{cccc}
\mathrm{S}_{11} & \mathrm{~S}_{12} & \ldots & \mathrm{S}_{1 \mathrm{~N}} \\
\mathrm{~S}_{21} & & & \vdots \\
\vdots & & & \\
\mathrm{S}_{\mathrm{N} 1} & \ldots & & \mathrm{S}_{\mathrm{NN}}
\end{array}\right]\left[\begin{array}{c}
\mathrm{V}_{1}^{+} \\
\mathrm{V}_{2}^{+} \\
\vdots \\
\mathrm{V}_{\mathrm{N}}^{+}
\end{array}\right]
$$

where the ij element of the $\mathrm{S}$ matrix is defined in Eq. 3.2. $\mathrm{S}_{\mathrm{ij}}$ is found by driving port $j$ with an incident wave of voltage $\mathrm{V}_{j}^{+}$, and measuring the reflected wave amplitude $\mathrm{V}_{\mathrm{i}}^{-}$, coming out of port i. All other ports must be terminated in matched loads to avoid reflection.

$$
\mathrm{S}_{\mathrm{ij}}=\left.\frac{\mathrm{V}_{\mathrm{i}}^{-}}{\mathrm{V}_{\mathrm{j}}^{+}}\right|_{\mathrm{V}_{\mathrm{k}}^{+}}=0 \text { for } \mathrm{k} \neq \mathrm{j}
$$


In principle, the 1.6 cell rf gun, see Fig. 3.1, can be considered a passive two port device. This allows us to probe the rf characteristic of the 1.6 cell rf gun using both $\mathrm{S}_{11}$ and $\mathrm{S}_{21}$ measurements, where port 1 is the rf waveguide feed into the full cell and port 2 is the rf pickup loop located in the vacuum port line.

\section{PORT 1}

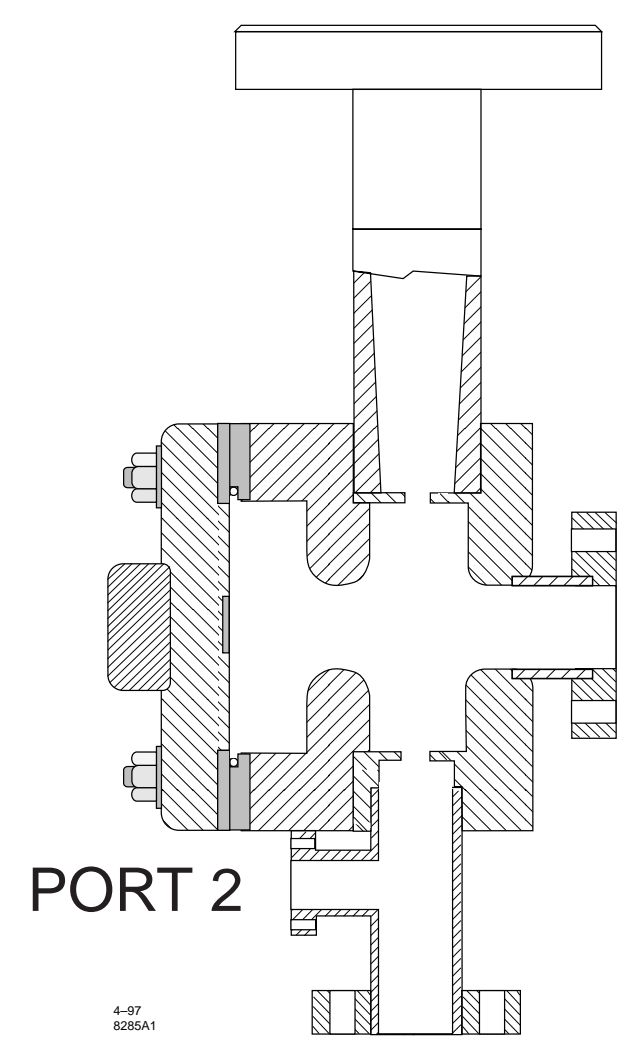

Figure 3.1: 1.6 cell rf gun represented as a two port device.

\subsection{2 rf Coupling Constant, $\beta_{\mathrm{rf}}$}

The rf coupling constant, $\beta_{\mathrm{rf}}$, is defined by Ginzton [35] by the introduction of two new additional definitions of the $\mathrm{Q}$ factors. The first of these new $\mathrm{Q}$ factors is the $\mathrm{Q}$ of the entire system, $\mathrm{Q}_{\mathrm{L}}$, which includes all sources of energy loss for the entire system. The second $\mathrm{Q}$ factor is that of just the cavity itself, $\mathrm{Q}_{0}$, which includes all 
sources of energy loss in the cavity. Eq. 3.3 is used to define the rf coupling constant, $\beta_{\mathrm{rf}}$.

$$
1+\beta_{\mathrm{rf}}=\frac{\mathrm{Q}_{\mathrm{o}}}{\mathrm{Q}_{\mathrm{L}}}
$$

We will discuss three ways to experimentally measure the rf coupling constant, $\beta_{\mathrm{rf}}$, between the source and the load for the $\pi$-mode of the rf gun. These are VSWR, $\mathrm{Q}_{\mathrm{L}}$, and Smith Chart reflection measurements.

\section{VSWR}

With the advent of modern network analyzers such as the HP-8510C, the use of the voltage standing wave ratio (VSWR) technique to measure $\beta_{\mathrm{rf}}$ has become antiquated. In this section we shall discuss the VSWR technique for completeness.

The VSWR is defined as the ratio of the voltage maximum, $\mathrm{V}_{\max }$, divided by the voltage minimum, $\mathrm{V}_{\text {min }}$, defined in Eq. 3.4

$$
\operatorname{VSWR}=\frac{\mathrm{V}_{\max }}{\mathrm{V}_{\min }}=\frac{\left|\mathrm{V}^{+}\right|+\left|\mathrm{V}^{-}\right|}{\left|\mathrm{V}^{+}\right|-\left|\mathrm{V}^{-}\right|}
$$

In Eq. 3.4, we have also written the VSWR in terms of $\mathrm{V}^{+}$and $\mathrm{V}^{-}$, which are the incident and reflected waves, respectively. In the case where the load impedance is purely resistive, it has been shown by Ginzton [35] that the VSWR can be written in the following form.

$$
\begin{array}{ll}
\text { if } \mathrm{R}_{\mathrm{L}}>\mathrm{Z}_{\mathrm{o}} & \text { then VSWR }=\frac{\mathrm{R}_{\mathrm{L}}}{\mathrm{Z}_{\mathrm{o}}} \\
\text { if } \mathrm{R}_{\mathrm{L}}<\mathrm{Z}_{\mathrm{o}} & \text { then VSWR }=\frac{\mathrm{Z}_{\mathrm{o}}}{\mathrm{R}_{\mathrm{L}}}
\end{array}
$$

where $Z_{0}$ is the impedance of the transmission line.

The absolute value of the coupling coefficient can be inferred by measuring the VSWR at resonance. The impedance at the voltage minimum and maximum are $\frac{Z_{o}}{\mathrm{VSWR}}$ and $Z_{\mathrm{o}} \mathrm{x}$ VSWR, respectively. $\beta_{\mathrm{rf}}$ is given by Eq. 3.6, depending on whether the system is under-coupled or over-coupled.

$$
\beta_{\mathrm{rf}}=\begin{array}{cl}
\text { vswr } & \text { over }- \text { coupled } \\
\frac{1}{\mathrm{vswr}} & \text { under }- \text { coupled }
\end{array}
$$


Mapping out the voltage standing wave pattern around the position of the detuned short, when the cavity is on resonance, one can determine if the cavity is under or over-coupled. If a voltage minimum is located at the detuned short position then the cavity is under-coupled. If a voltage maximum is located at the detuned short position then the cavity is over-coupled.

\section{Transmission Method}

Since the 1.6 cell rf gun is a two-port device, we can in principle conduct a transmission measurement. With knowledge of the resonant frequency and the frequencies of the $-3 \mathrm{~dB}$ power points, we can calculate the loaded Q, $\mathrm{Q}_{\mathrm{L}}$. From SUPERFISH [5] we know what the unloaded $\mathrm{Q}, \mathrm{Q}_{\mathrm{o}}$, should be. Therefore we can use Eq. 3.7 to calculate the coupling coefficient $\beta_{\mathrm{rf}}$.

$$
1+\beta_{\mathrm{rf}}=\frac{\mathrm{Q}_{\mathrm{o}}}{\mathrm{Q}_{\mathrm{L}}}
$$

The transmission method was not used to conduct the final tuning of the rf gun, since the calculated value of $Q_{0}$ does not take into account surface finish, braze joints, or variations in the conductive of the rf gun material.

\section{Smith Chart}

The Smith Chart representation of the reflection coefficient, $\mathrm{S}_{11}$, is a direct measurement of the $\beta_{\mathrm{rf}}$. This technique was utilized during the tuning of the OFHC Cu 1.6 rf gun. Using the Smith Chart representation and a properly calibrated network analyzer, it is possible to directly measure $\mathrm{Q}_{\mathrm{o}}, \mathrm{Q}_{\mathrm{L}}$, and $\mathrm{Q}_{\mathrm{ext}}$ as given in Eq. 3.8.

$$
\begin{aligned}
& Q_{0}=\frac{f_{o}}{f_{1}-f_{2}} \\
& Q_{\text {ext }}=\frac{f_{o}}{f_{3}-f_{4}} \\
& Q_{\mathrm{L}}=\frac{f_{o}}{f_{5}-f_{6}}
\end{aligned}
$$

Where the $\mathrm{f}_{\mathrm{o}}$ is the $\pi$-mode resonant frequency into a purely resistive load.

$\mathrm{f}_{1}$ and $\mathrm{f}_{2}$ are frequencies determined from the intersection of the $\mathrm{S}_{11}$ and the $\mathrm{r}=\mathrm{x}$ line. 
$f_{3}$ and $f_{4}$ are frequencies determined from the intersection of the $S_{11}$ and the $\mathrm{x}= \pm 1$ line.

$\mathrm{f}_{5}$ and $\mathrm{f}_{6}$ are frequencies determined from the intersection of the $\mathrm{S}_{11}$ and the $\mathrm{x}=\mathrm{r} \pm 1$ line.

$z_{0}=r \pm i x$ is the impedance loci of the $S_{11}$ response curve on a normalized Smith Chart. These seven frequency positions are shown in Fig. 3.2.

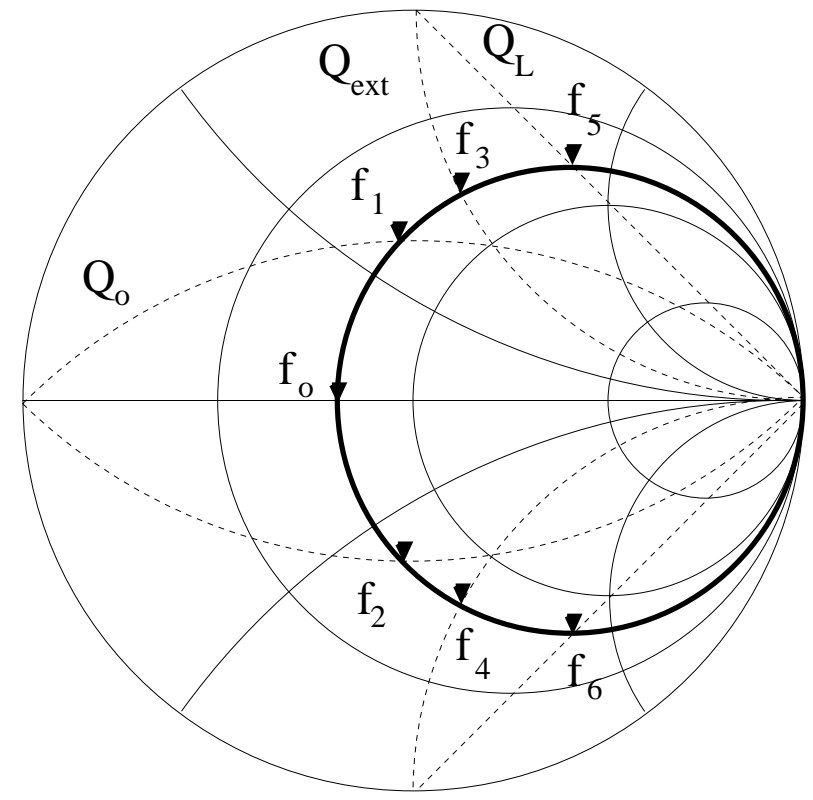

Figure 3.2: Smith Chart representation of a over-coupled system.

Measuring these seven frequency values, it is possible to directly calculate $\beta_{\mathrm{rf}}$ utilizing Eq. 3.9, 3.10, and 3.11.

$$
\begin{gathered}
\frac{1}{\mathrm{Q}_{\mathrm{L}}}=\frac{1}{\mathrm{Q}_{\mathrm{o}}}+\frac{\beta_{\mathrm{rf}}}{\mathrm{Q}_{\mathrm{o}}} \\
\frac{1}{\mathrm{Q}_{\mathrm{L}}}=\frac{1}{\mathrm{Q}_{\mathrm{o}}}+\frac{1}{\mathrm{Q}_{\mathrm{ext}}} \\
\beta_{\mathrm{rf}}=\frac{\mathrm{Q}_{\mathrm{o}}}{\mathrm{Q}_{\mathrm{ext}}}
\end{gathered}
$$




\subsubsection{Bead Perturbation}

The Slater Perturbation Theorem [36] is the underlying theory used to probe the electro-magnetic field in a standing wave rf cavity. We can see in Eq. 3.12 that measuring the frequency shift between the perturbed resonant frequency, $\omega$, and unperturbated resonant frequency, $\omega_{0}$, we can directly measure the difference between the square of the magnetic field, $\mathbf{H}_{\alpha}$, and the electric field, $\mathbf{E}_{\alpha}$, at the position of the perturbing object, indicated in Eq. 3.12 by $\delta \tau$.

$$
\omega^{2}=\omega_{\mathrm{o}}^{2}\left[1+\frac{\int_{\delta \tau}\left\{\mathbf{H}_{\alpha}{ }^{2}-\mathbf{E}_{\alpha}{ }^{2}\right\} \mathrm{d} \tau}{\int_{\delta \mathrm{v}}\left\{\mu \mathbf{H}^{2}+\epsilon \mathbf{E}^{2}\right\} \mathrm{dv}}\right],
$$

where $\mu$ and $\epsilon$ are the permeability and permittivity of free space, respectively. $\mathbf{H}_{\alpha}$ and $\mathbf{E}_{\alpha}$ are proportional to the magnetic and electric field vectors at the perturbing object location, indicated by $\delta \tau . \mathbf{H}_{\alpha}$ and $\mathbf{E}_{\alpha}$ are dependent on the perturbing object's volume, shape, composition, and location through the perturbing object's electric and magnetic polarizabilities. $\mathbf{H}$ and $\mathbf{E}$ are the magnetic and electric field vectors in the unperturbed cavity volume, indicated by $\delta \mathrm{v}$.

Eq. 3.12 can be cast in a more useful form when we recognize that the integral in the denominator is equal to $8 \pi$ times the total stored energy in the cavity, u. Therefore, Eq. 3.12 becomes

$$
\frac{\omega^{2}-\omega_{0}^{2}}{\omega_{\mathrm{o}}^{2}}=\frac{1}{8 \pi \mathrm{u}} \int_{\delta \tau}\left\{\mathbf{H}_{\alpha}{ }^{2}-\mathbf{E}_{\alpha}{ }^{2}\right\} \mathrm{d} \tau .
$$

Utilizing the proper placement and shape selection of the perturbing object, we can in principle eliminate all components of the electro-magnetic field that contribute to the frequency shift except for the field component of interest. This allows us to combine the perturbing object's remaining electric or magnetic polarizabilities component with the normalizing term. Combining the single remaining component of the electric or magnetic polarizability into the normalizing constant is allowed only if we are interested in the relative field strength of one component of the electromagnetic field.

For example, the on axis accelerating field, $\mathrm{E}_{\mathrm{z}}$, can be studied by placing a small perturbing sphere made of a known dielectric material at a set of known positions 
along the beam axis. Measuring the frequency shift at each location, the relative field amplitudes are found using Eq. 3.14.

$$
\mathrm{E}_{\mathrm{z}} \propto \sqrt{\omega_{\mathrm{o}}^{2}-\omega^{2}}
$$

Since the bead size effects both the amount of the frequency shift and also the components of the electro-magnetic field that are studied, the size of the perturbing object is a major consideration. However, the placement of the perturbing object allows for the elimination of all other electro-magnetic field components other than $E_{z}$, since $E_{z}$ is the only electro-magnetic field that is present on-axis in a $\mathrm{TM}_{010}$ mode cavity in the limit as the sphere radius goes to zero.

The shape of the perturbing object can also result in the elimination of unwanted field components. For example, if a metallic needle were placed in the middle of an accelerating cavity, with its long axis parallel with the beam axis but displaced a distance $\mathrm{R}$ in the radial direction from the beam axis, the $\mathrm{E}_{\mathrm{z}}$ component will be perturbed to a greater degree than that of $\mathrm{H}_{\phi}$. The placement in the middle of the cavity will eliminate the $\mathrm{E}_{\mathrm{r}}$ component. This is the technique used in Section 3.7.2 to measure $\mathrm{E}_{\mathrm{z}}$ as a function of $\theta$.

\subsection{6 Cell rf Gun Design}

In this section we shall discuss the general $\mathrm{rf}$ and beam dynamics design issues that dictated the mechanical design of the rf gun. The manufacturing techniques, mechanical tolerances, and cleaning issues will also be discussed. Fig. 3.3 is a cut away view of the gun in the $\mathrm{Y}-\mathrm{Z}$ plane.

\subsubsection{General rf and Beam Dynamics Design Issues}

Previous BNL type rf guns used magnetic side coupling to provide rf power into both the full and half cell of the rf gun. From symmetry arguments it can be shown that this scheme suppresses the zero mode. This is demonstrated in Fig. 3.4 where the $\mathrm{TE}_{01}$ waveguide mode couples its magnetic field into both the full and half cell. 


\subsection{Cell RF Gun}

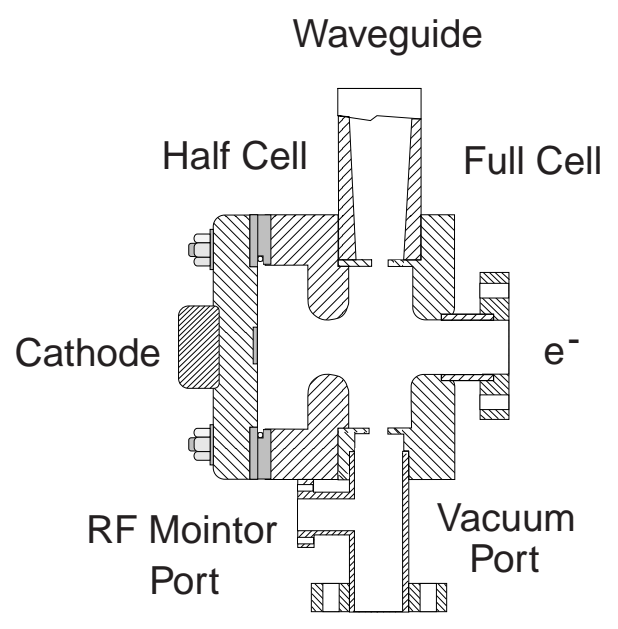

Figure 3.3: 1.6 cell rf gun cross sectional view.

The magnetic field in the cells of the rf gun will have the opposite sense. This induces an electric field that has the opposite orientation between the full and half cell. This is the field distribution of the $\pi$-mode. The 0 -mode is suppressed since there is no magnetic field component in both the full and half cells that have the same sense. The 1.6 cell rf gun also uses magnetic coupling, but only into the full cell. Cell-to-cell coupling is utilized to couple rf power between the full and half cells. In this case there is no preferred magnetic field orientation. The resonant frequency of the rf power and the $\pi$-mode will determine the preferred mode of operation.

To make this viable, the aperture between the half cell and the full cell was increased. This increased the coupling between the two cells and therefore increased the separation in frequency between the 0 -mode and the $\pi$-mode, so that the suppression of the 0-mode is not necessary.

All previous BNL type rf guns have had their performance limited by the asymmetric coupling of rf power into the gun. We have corrected this asymmetry by symmetrizing the rf coupling hole into the full cell with a vacuum port of the same dimensions as the rf coupling located on the opposite side of the full cell.

All other cavity penetrations into both the full and half cell have also been symmetrized for the 1.6 cell rf gun. There are two plunger type tuners located in the 


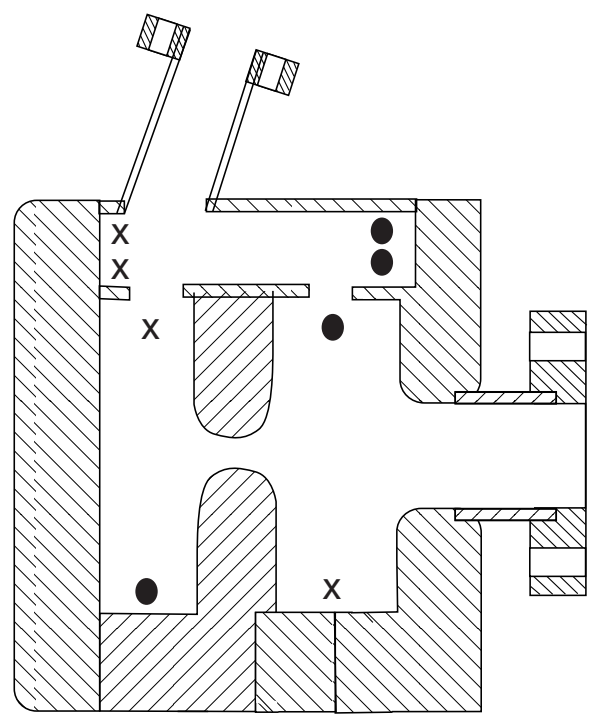

$X \quad$ Magnetic Field into the page

- Magnetic Field out of the page

Figure 3.4: 0-mode suppression in a magnetically side coupled BNL type rf gun.

full cell. They are located opposite each other and rotated $90^{\circ}$ with respect to the rf/vacuum plane. There are symmetric grazing incident laser ports in the half cell which, besides symmetrizing the electric field in the half cell, also allow the reflected laser pulse to exit the half cell. This eliminates the problem of photoemission due to multiple reflections of the laser pulse. The laser ports are race track in shape, with the long axis parallel to the beam axis. This allows varying the angle of incidence of the laser pulse with respect to the normal vector projecting from the cathode plane. The orientation of all of the cavity penetrations will become critical in our experimental investigation of electro-magnetic asymmetries in Chapter 5. Therefore, it needs to be pointed out that the laser ports are rotated $-45^{\circ}$ with respect to the $+\mathrm{X}$ axis shown in Fig 3.5.

Another major design change from previous generations of BNL type rf guns was the placement of the cathode with respect to the half cell $\mathrm{rf}$ and vacuum joints. The first generation BNL rf gun, Gun 1A, utilized a cathode plug $2.5 \mathrm{~cm}$ in diameter which allowed for the insertion of various cathode materials into the gun. This allowed for quantum efficiency studies utilizing various materials such as copper and magnesium. 


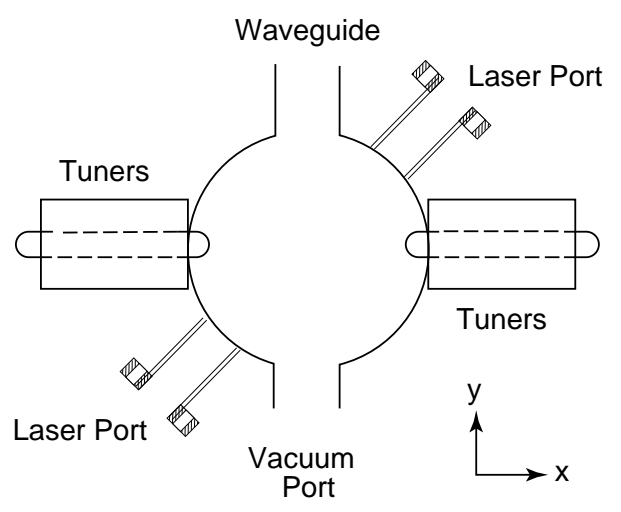

Figure 3.5: Cavity penetration into the 1.6 cell rf gun, as seen from the downstream end of the rf gun.

This cathode plug limited the maximum field gradient that could be held off due to multipacting in the cathode plug/plate interface. To eliminate this problem, a second generation BNL rf gun was developed. Gun 1B, eliminated the multipacting problem but did not allow for cathode replacement.

In the 1.6 cell rf gun design, the capability of cathode replacement was of critical importance but the multipacting problem also needed to be eliminated. To accomplish both of these objectives simultaneously the cathode plug was eliminated and replaced by a removable cathode plate. The rf and vacuum joint is made by a helico flex seal located at the outer diameter of the half cell. This moved the cathode joint from a high field and low current region to a low field and high current region of the half cell, thereby eliminating the multipacting problem and still allowing for cathode replacement.

The BNL/SLAC/UCLA 1.6 cell S-band rf gun, as its name implies, has had its half cell lengthened to provide more rf focusing in the low energy region of the gun and to decrease the cathode to iris electric field ratio. The half cell lengthening decreased the cathode to iris field ratio from 1.10 to 1.06. This allows for higher operational field gradients with reduced dark current levels from the cell-to-cell iris.

Resistive heating of the gun body is used to stabilize its operating temperature. This technique utilizes the environment as a heat sink. That is, energy is transfered from the gun into the environment as would be the case of a high duty cycle water 
cooled rf gun which transfers energy from the gun body into the cooling water. Experimental results indicate that the gun can operate up to $10 \mathrm{~Hz}$ at $15 \mathrm{MW}$ of input power with a $4 \mu \mathrm{sec}$ rf pulse length utilizing the surrounding environment as a heat sink. For energy levels above this limit, for example with the Next Generation Photoinjector (NGP), some other type of cooling must be used, since the total energy loss will exceed the environment's ability to absorb this energy. The NGP was designed to operate at $55^{\circ} \mathrm{C}$, since the environmental temperature at Brookhaven Accelerator Test Facility during the summer can reach $37^{\circ} \mathrm{C}$.

\subsubsection{Manufacturing Techniques and Tolerances}

The 1.6 cell rf gun body is composed of grade II oxygen free high conductivity copper (OFHC II). Aluminum machining fixtures were not allowed to be used, to prevent the introduction of field emitting sites on the rf surfaces. Aluminum contamination of the brazing surfaces was prevented by manufacturing procedures. New machine tool bits were procured for the manufacture of all the gun parts. This prevented the introduction of unknown contaminants that might be embedded in the cutting surface of a used tool bit. The desired surface finish was attained using a copper wool polishing technique.

$$
\mathrm{FB} \equiv \frac{\mathrm{E}_{2}}{\mathrm{E}_{1}}
$$

The rf gun was designed to have balanced fields with a critically coupled $\pi$-mode at $2856 \mathrm{MHz}$. Field Balance (FB) is defined in Eq. 3.15 as the ratio of the peak accelerating field in the middle of the full cell divided by the cathode field in the half cell. The dimensions of the gun were set by low power rf measurements of a brass prototype with $\beta_{\mathrm{rf}}=0.50$. Scaling the difference of resistivity between the free cutting brass and OFHC II Cu, the rf gun coupling constant should have been $\beta_{\mathrm{rf}}=1.00$. The rf characteristics of the gun were checked after all machining was completed at UCLA. The OFHC II Cu rf gun was found to be significantly under-coupled, with $\beta_{\mathrm{rf}}=0.54$. Fig. 3.6 is a mechanical drawing of the gun, with its pertinent mechanical dimensions labeled. These labels correspond to the final achieved mechanical 
dimensions in Table 3.1 with their respective tolerances.

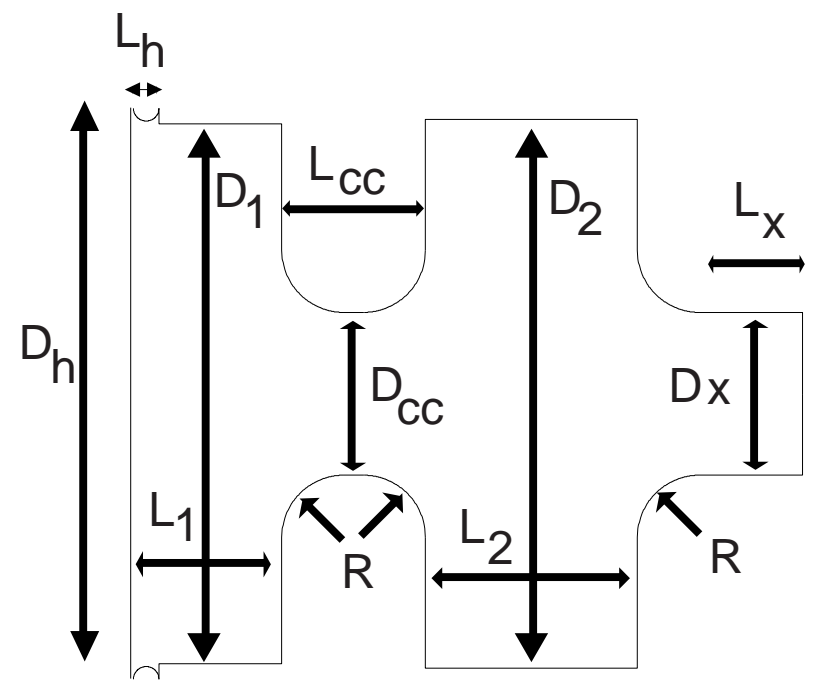

Figure 3.6: Dimension labels corresponding to Table 3.1.

During the initial frequency test of the full cell at SLAC and after rough machining at UCLA, it was determined that the frequency of the full cell was too high. Material from the inductive region of the full cell side of the cell-to-cell iris was removed. This decreased the frequency of the full cell without effecting the thickness of the rf coupling slot at the outer diameter (OD) of the full cell, which would have increased the $\beta_{\mathrm{rf}}$ of the $\pi$-mode. In retrospect, cutting on the OD to decrease the full cell independent frequency would have been the optimum choice, if we had known that the $\pi$-mode $\beta_{\text {rf }}$ was too low.

After the final rf tuning of the full cell, the 1.6 cell rf gun was brazed together in a $\mathrm{H}_{2}$ brazing furnace in a two temperature process. First, the full cell, half cell, and stainless steel (SST) half cell seal plate were brazed together along with ancillary components that consist of two SST laser ports, two SST tuner tubes, and the SST beam exit port. At the same brazing temperature but in a smaller $\mathrm{H}_{2}$ furnace, the cathode plate was brazed together with the SST cathode helico flex sealing surface and SST anti-torque nut. Finally, the SST vacuum short tube was brazed to the SST rf monitor loop tube. The brazing material in this step was a 35-65 $\operatorname{gold}(\mathrm{Au})-\operatorname{copper}(\mathrm{Cu})$ alloy with a wetting temperature of $1025^{\circ} \mathrm{C}$. All SST used in the construction of the 


\begin{tabular}{|l|l|}
\hline Label & Dimensions (inch) \\
\hline $\mathrm{D}_{1}$ & $3.249 \pm_{0.001}^{0.000}$ \\
\hline $\mathrm{L}_{1}$ & $0.908 \pm 0.005$ \\
\hline $\mathrm{D}_{2}$ & $3.289 \pm_{0.001}^{0.000}$ \\
\hline $\mathrm{L}_{2}$ & $1.279 \pm 0.001$ \\
\hline $\mathrm{R}$ & $0.375 \pm 0.001$ \\
\hline $\mathrm{D}_{\mathrm{x}}$ & $0.984 \pm_{0.000}^{0.001}$ \\
\hline $\mathrm{L}_{\mathrm{x}}$ & $1.535 \pm 0.006$ \\
\hline $\mathrm{D}_{\mathrm{cc}}$ & $0.984 \pm_{0.000}^{0.001}$ \\
\hline $\mathrm{L}_{\mathrm{cc}}$ & $0.868 \pm 0.005$ \\
\hline $\mathrm{D}_{\mathrm{h}}$ & $3.268 \pm 0.002$ \\
\hline $\mathrm{L}_{\mathrm{h}}$ & $0.154 \pm 0.002$ \\
\hline
\end{tabular}

Table 3.1: 1.6 cell rf gun mechanical dimensions and tolerances for Fig. 3.6.

1.6 cell rf gun was tested to be non-magnetic.

A cut and measure technique was used to conduct the final tuning of the half cell of the 1.6 cell rf gun after the first braze step. To facilitate this, the tuner assemblies were constructed and the ConFlat flanges were spot welded onto the non-magnetic SST tuner tubes. This allowed precise and repeatable positioning of the tuners from cut to cut on the half cell. Using a copper electrode, a sinker Electro Discharge Machining (EDM) cut was conducted on the rf and vacuum coupling iris of the full cell. This was done to increase the rf coupling constant, $\beta_{\mathrm{rf}}=0.54$. Fig. 3.7 represents the coupling iris dimensions after EDM. A significant frequency decrease was observed with a slight increase in the rf coupling constant, $\beta_{\mathrm{rf}}=0.71$. Further use of EDM to increase the rf coupling slot size was rejected because of the strong $\beta_{\mathrm{rf}}$ and the full cell frequency dependence. It was decided to use an inductive post in the waveguide assembly to match the rf to the $\pi$-mode without effecting the full cell frequency.

When the gun was matched with $\beta_{\mathrm{rf}}=1.05$, balanced fields, and the $\pi$-mode at $2856 \mathrm{MHz}$, the second and final braze was conducted using a 50-50 gold copper alloy which wets at a temperature of $982^{\circ} \mathrm{C}$. The rf gun body and the wave guide assembly along with the inductive matching post and the vacuum tube assembly were brazed together. This complicated system of parts was oriented vertically, using gravity, which allowed the assembly to be self jigging. 


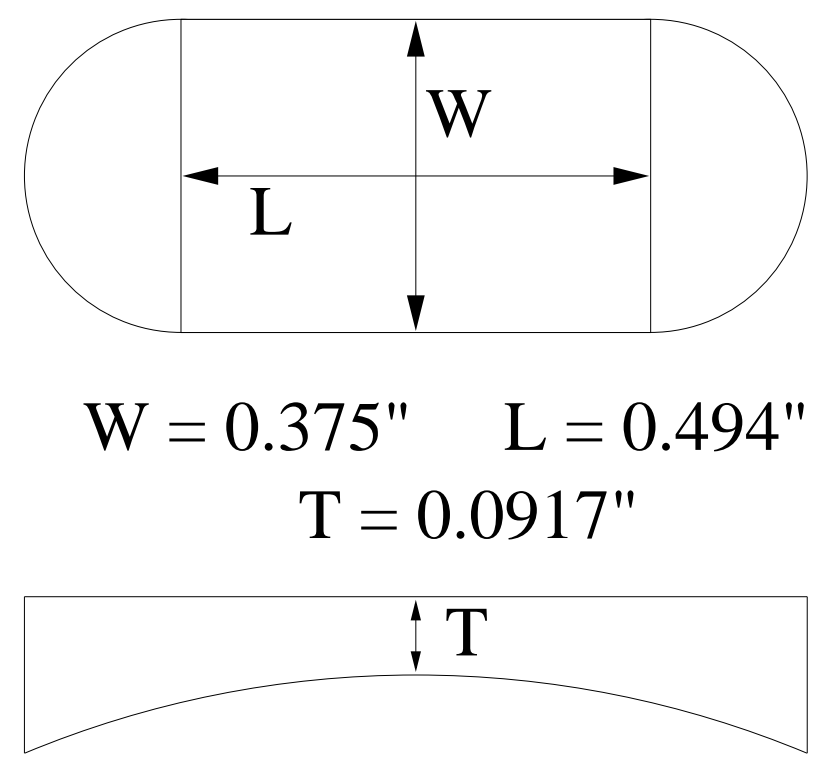

Figure 3.7: rf and vacuum iris dimensions after EDM.

\subsubsection{Cleaning Issues}

To prevent vacuum contamination, the only machining lubricant used during the rough machining was Kool Mist 77, in a flushed and filtered recirculation system. For the final machining, ethanol was the only lubricant authorized for use. Clean nylon gloves were used in the handling of the gun parts during all stages of the manufacturing process. This prevented the introduction of body acids onto the $\mathrm{rf}$ gun parts. When the gun parts were not being machined they were triple-wrapped in lint-free cloth and oil-free Ultra High Vacuum (UHV) Aluminum (Al) foil for storage.

When all the gun parts were completed, the parts were cleaned at SLAC, using the SLAC UHV/brazing cleaning process [37]. Also high pressure (HP) air was not used at any time to blow off the parts. Bottled $\mathrm{N}_{2}$ was used, since foreign particles can be expelled from the HP nozzle and cause considerable damage to the rf gun parts.

A vacuum bake-out was conducted on the fully brazed gun body, cathode plate, and ancillary equipment at $450^{\circ} \mathrm{C}$ for 10 days. This removed excess $\mathrm{H}_{2}$ that is introduced into the OFHC II $\mathrm{Cu}$ during the brazing cycles. The base pressure attained was $6 \times 10^{-9}$ Torr at $450^{\circ} \mathrm{C}$. At room temperature, the ultimate base pressure attained 


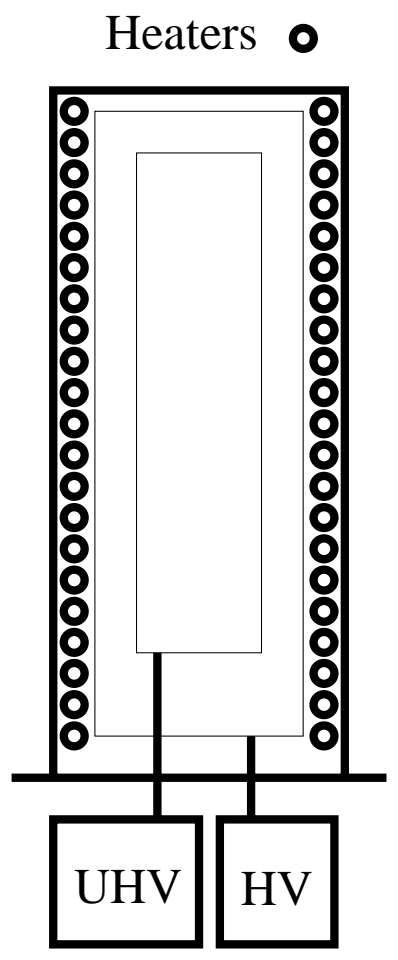

Figure 3.8: Schematic diagram of UHV bake-out stand. 
was $9 \times 10^{-10}$ Torr.

A schematic diagram of the bake-out station is shown in Fig. 3.8. The gun components were located in the UHV chamber. Both the inner and outer surfaces of the rf gun were baked and pumped on during the high temperature bake-out. The tuners' rf spring fingers, composed of a beryllium copper alloy, would have lost their tensile strength if baked out. Therefore, the rf spring fingers were not baked-out. In principle, we would have preferred to fully assembly the rf gun and bake-out only the inner surfaces of the rf gun. This would have decreased the time needed to reach the final base pressure of $6 \times 10^{-9}$ Torr while at a temperature of $450^{\circ} \mathrm{C}$. Spin polarized electron sources need to attain base pressures of $10^{-12}$ Torr. This requires that a spin polarized rf gun be baked out fully assembled and never exposed to the atmosphere. The loss of tensile strength of the tuners' rf spring fingers will have to be addressed before a spin polarized rf gun is feasible. Perhaps rf choke joints could be used instead of spring fingers. Thereby eliminating the problem of the loss of tensile strength of the beryllium copper alloy. The 1.6 cell rf gun ultimate base pressure after rf conditioning was $10^{-10}$ Torr.

\subsection{Electro-magnetic Simulations}

SUPERFISH [5], a two-dimensional electro-magnetic field solver, was used to simulate the electro-magnetic field in the $\mathrm{rf}$ gun. These fields were mapped into PARMELA [3] to more accurately represent the rf gun field and hence the accuracy of the beam dynamics simulations discussed in Chapter 2. Fig. 3.9 represents the $\pi$ and 0-modes field maps produced by SUPERFISH [5]. Simulation results of the $\pi$ and 0-mode are presented in Table 3.2.

Frequency scaling laws for cavity machining were also developed from these simulations. During the final gun tuning three major dimensions were machined: the full cell outer diameter (OD), half cell OD, and the half cell length. Both the full and half cell OD frequency change can be understood from an LC oscillator model. As the magnetic field volume increases the inductance increases; therefore, the resonant frequency will decrease. But the half cell length is not as straightforward: As the 


\begin{tabular}{|l|l|}
\hline Parameter & SUPERFISH \\
\hline$\left(\frac{\mathrm{E}_{2}}{\mathrm{E}_{1}}\right)_{\pi}$ & 1.00146 \\
\hline $\mathrm{f}_{\pi}$ & $2856.04994 \mathrm{MHz}$ \\
\hline $\mathrm{Q}_{\mathrm{o}, \pi}$ & 15335 \\
\hline $\mathrm{R}_{\mathrm{s}, \pi}$ & $46 \frac{\mathrm{M} \Omega}{\mathrm{m}}$ \\
\hline$\left(\frac{\mathrm{E}_{2}}{\mathrm{E}_{1}}\right)_{0}$ & 0.571 \\
\hline $\mathrm{f}_{0}$ & $2852.58589 \mathrm{MHz}$ \\
\hline $\mathrm{Q}_{\mathrm{o}, 0}$ & 12920 \\
\hline $\mathrm{R}_{\mathrm{s}, 0}$ & $42 \frac{\mathrm{M} \Omega}{\mathrm{m}}$ \\
\hline$\delta \mathrm{f}$ & $3.46405 \mathrm{MHz}$ \\
\hline Length & $15 \mathrm{~cm}$ \\
\hline
\end{tabular}

Table 3.2: SUPERFISH simulations results of the 1.6 cell $\mathrm{rf}$ gun, for $\pi$-mode balanced fields.
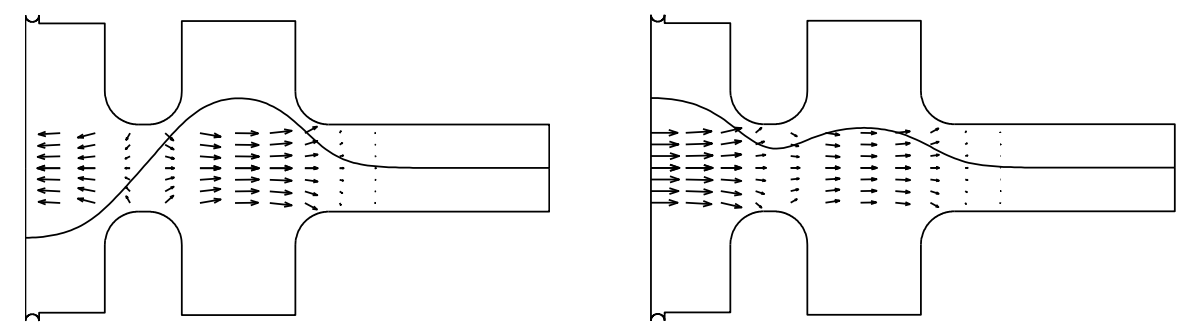

$$
\mathrm{f}_{\pi}=2856.050 \mathrm{MHz} \quad \mathrm{f}_{\mathrm{o}}=2852.586 \mathrm{MHz}
$$

Figure 3.9: $\pi$-mode and 0-mode $\mathrm{E}_{\mathrm{z}}$ field profiles for a balance $\pi$-mode at $2856 \mathrm{MHz}$. 


\begin{tabular}{|c|c|}
\hline Parameter & SUPERFISH \\
\hline$\frac{\mathrm{df}_{\pi}}{\mathrm{dD}_{2}}$ & -0.579 \\
\hline$\frac{\mathrm{df}_{2}}{\mathrm{dD}_{2}}$ & -0.906 \\
\hline$\frac{\mathrm{df}_{\pi}}{\mathrm{dD}_{1}}$ & -0.270 \\
\hline$\frac{\mathrm{df}_{1}}{\mathrm{dD}_{1}}$ & -0.742 \\
\hline$\frac{\mathrm{df}_{\pi}}{\mathrm{dL}_{1}}$ & -0.051 \\
\hline$\frac{\mathrm{d}_{1}}{\mathrm{dL}_{1}}$ & -0.138 \\
\hline
\end{tabular}

Table 3.3: Detuning effect due to cavity dimensional changes, in units of $\frac{\mathrm{MHz}}{\mathrm{mill}}$. The cavity dimensions correspond to Fig. 3.6.

length of the cavity decreases the inductance will decrease but the capacitance will increase. These two effects counter each other with respect to the resonant frequency of the half cell. SUPERFISH [5] was used to study this detuning effect and it was found that the magnetic field contribution dominates over the electric field contribution. Frequency detuning results from SUPERFISH [5] simulations of the 1.6 cell rf gun cavity are presented in Table 3.3 .

\subsubsection{Field Balance Versus Mode Separation}

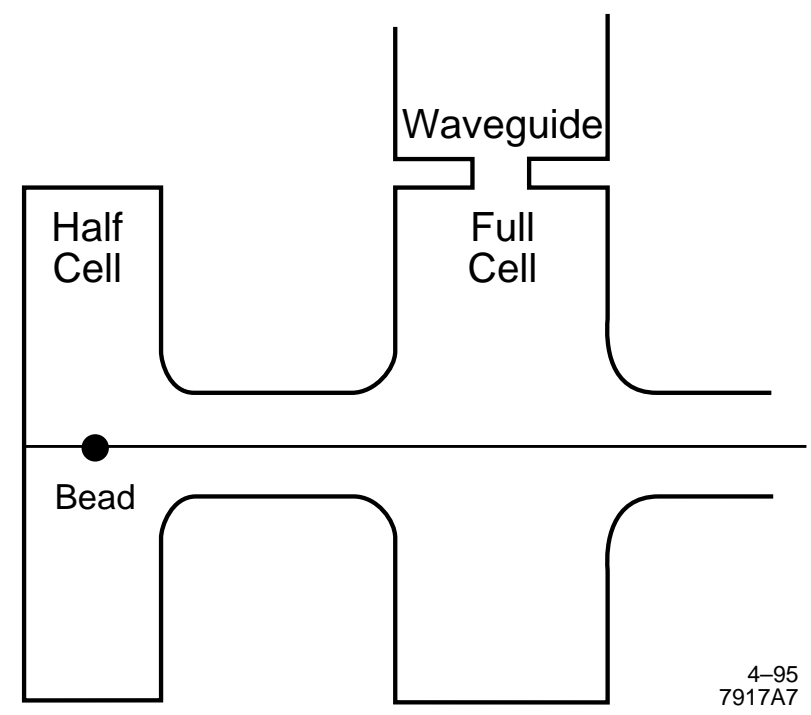

Figure 3.10: Bead pull experimental setup. 
The full cell to half cell coupling is vital since side coupling to the half cell was not used in the 1.6 cell rf gun design. We further improved the technique of using mode separation for field balance tuning [38]. To measure the field balance versus mode separation it is necessary to measure $E_{z, \pi}$ for different mode separations, $\delta f=f_{\pi}-f_{0}$. This is accomplished by changing the full and half cell independent frequencies, using the tuners located in both cavities of the low power rf brass model. The experimental setup for the longitudinal bead pull used to measure the field balance is illustrated in Fig. 3.10. For large differences in the full and half cell resonant frequencies, the two observed frequencies are essentially the independent cell resonant frequencies. When the independent cell frequencies are within a Q width of each other, the coupling splits the cell frequencies into the $\mathrm{f}_{\pi}$ and $\mathrm{f}_{0}$.

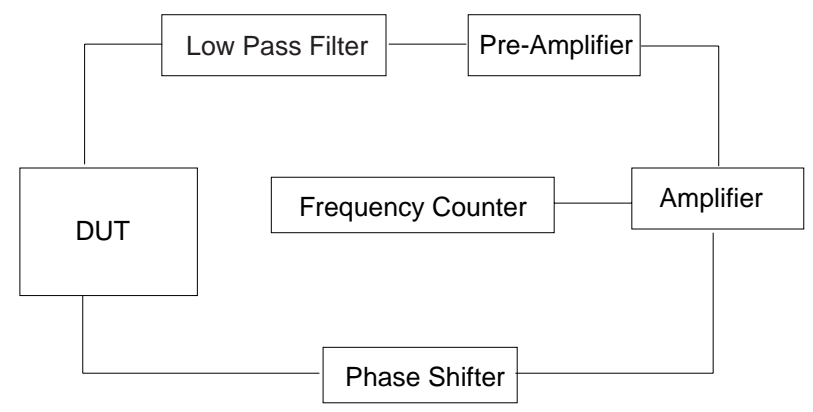

Figure 3.11: Self excited loop used to increase the frequency resolution of our field measurements.

On axis $\mathrm{E}_{z}$ data was taken using a $4.72 \mathrm{~mm}$ diameter dielectric sphere and a self excited loop instead of a network analyzer, see Fig 3.11. This technique increased the accuracy of the frequency measurement down to $100 \mathrm{~Hz}$, over that what could be attained from the HP-8510A used at the Brookhaven Accelerator Test Facility (ATF). The predictions of our equivalent circuit LC model, SUPERFISH [5], MAFIA [39], and our experimental data is presented in Fig. 3.12.

\section{Equivalent Circuit LC Model}

When the 1.6 cell rf gun is being tuned up for high power operation, conducting a longitudinal bead pull is not possible. Therefore, some indirect way to measure the field balance is needed. 


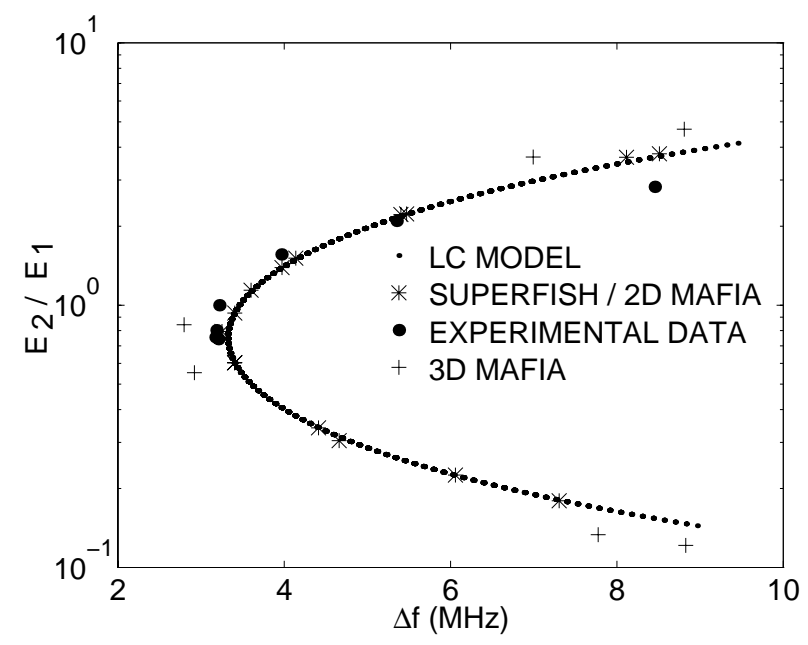

Figure 3.12: Field balance versus mode separation.

An equivalent circuit LC model of the cell-to-cell coupling was developed to fulfill this need. Our cell-to-cell model is shown in Fig. 3.13. The subscripts 1 and 2 represent the half and full cell, respectively. During development of this equivalent circuit model, measurements of $\mathrm{E}_{z}(\mathrm{z}, \mathrm{r}=0.00)$ as a function of mode separation were conducted to experimentally verify the accuracy of the equivalent circuit LC model. Numerical simulations were conducted on the equivalent circuit LC model of the cell-to-cell coupling whose development is shown below.

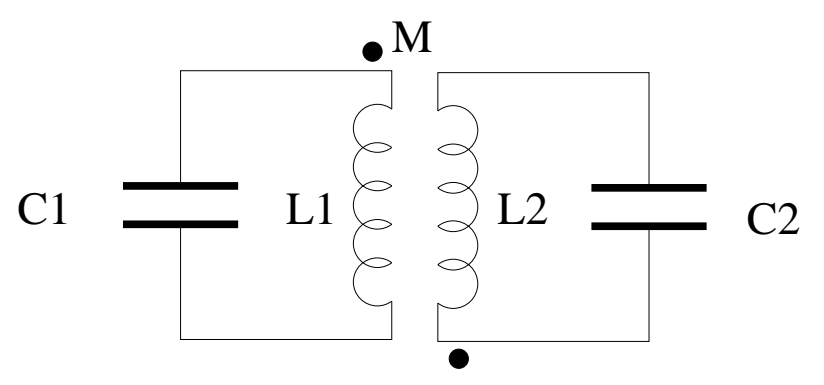

Figure 3.13: Equivalent circuit LC model for the cell-to-cell coupling.

The equations of motion of this system can be derived from elementary electric circuit theory but we proposed to solve for the equations of motion using Hamilton's principle [22]. 


$$
\begin{gathered}
\mathrm{H}=\mathrm{H}\left(\mathrm{q}_{\mathrm{k}}, \mathrm{p}_{\mathrm{k}}, \mathrm{t}\right) \\
\dot{\mathrm{p}}_{\mathrm{k}}=-\frac{\partial \mathrm{H}}{\partial \mathrm{q}_{\mathrm{k}}} \quad \dot{\mathrm{q}}_{\mathrm{k}}=\frac{\partial \mathrm{H}}{\partial \mathrm{p}_{\mathrm{k}}}
\end{gathered}
$$

Making the following identities

$$
\mathrm{p}_{\mathrm{k}}=\mathrm{L}_{\mathrm{k}} \mathrm{I}_{\mathrm{k}} \quad \mathrm{q}_{\mathrm{k}}=\mathrm{C}_{\mathrm{k}} \mathrm{V}_{\mathrm{k}},
$$

we can convert the total energy of the LC coupled circuit

$$
\mathrm{E}=\frac{\mathrm{L}_{1} \mathrm{I}_{1}^{2}}{2}+\frac{\mathrm{L}_{2} \mathrm{I}_{2}^{2}}{2}+\mathrm{MI}_{1} \mathrm{I}_{2}+\frac{\mathrm{C}_{1} \mathrm{~V}_{1}^{2}}{2}+\frac{\mathrm{C}_{2} \mathrm{~V}_{2}^{2}}{2}
$$

into the Hamiltonian of the system. $\mathrm{M}$ is the mutual inductance between $\mathrm{L}_{1}$ and $\mathrm{L}_{2}$, which models the cell-to-cell coupling. The amount of mutual inductance, $\mathrm{M}$, was adjusted to fit the minimum mode separation. Which was found experimental by conducting longitudinal field measurements. The mode separation, for the case of the $\pi$-mode with balanced fields, was found to be $3.225 \mathrm{MHz}$.

$$
\mathrm{H}=\frac{\mathrm{p}_{1}^{2}}{2 \mathrm{~L}_{1}}+\frac{\mathrm{p}_{2}^{2}}{2 \mathrm{~L}_{2}}+\frac{\mathrm{M}}{\mathrm{L}_{1} \mathrm{~L}_{2}} \mathrm{p}_{1} \mathrm{p}_{2}+\frac{\mathrm{q}_{1}^{2}}{2 \mathrm{C}_{1}}+\frac{\mathrm{q}_{2}^{2}}{2 \mathrm{C}_{2}}
$$

Solving for the Hamilton equations of motion of this system we find that

$$
\left[\begin{array}{c}
\dot{\mathrm{V}}_{1} \\
-\dot{\mathrm{I}}_{1} \\
\dot{\mathrm{V}}_{2} \\
-\dot{\mathrm{I}}_{2}
\end{array}\right]=\left[\begin{array}{cccc}
0 & \frac{1}{\mathrm{C}_{1}} & 0 & \frac{\mathrm{M}}{\mathrm{L}_{1} \mathrm{C}_{1}} \\
\frac{1}{\mathrm{~L}_{1}} & 0 & 0 & 0 \\
0 & \frac{\mathrm{M}}{\mathrm{L}_{2} \mathrm{C}_{2}} & 0 & \frac{1}{\mathrm{C}_{2}} \\
0 & 0 & \frac{1}{\mathrm{~L}_{2}} & 0
\end{array}\right]\left[\begin{array}{c}
\mathrm{V}_{1} \\
\mathrm{I}_{1} \\
\mathrm{~V}_{2} \\
\mathrm{I}_{2}
\end{array}\right]
$$

Now, relating the time derivative to $-\mathrm{j} \omega$ we have the generalized eigenvalue and eigenvector problem that can be solved by any number of numerical techniques.

$$
-\mathrm{j} \omega\left[\begin{array}{cccc}
-1 & 0 & 0 & 0 \\
0 & 1 & 0 & 0 \\
0 & 0 & -1 & 0 \\
0 & 0 & 0 & 1
\end{array}\right]\left[\begin{array}{c}
\mathrm{V}_{1} \\
\mathrm{I}_{1} \\
\mathrm{~V}_{2} \\
\mathrm{I}_{2}
\end{array}\right]=\left[\begin{array}{cccc}
0 & \frac{1}{\mathrm{C}_{1}} & 0 & \frac{\mathrm{M}}{\mathrm{L}_{1} \mathrm{C}_{1}} \\
\frac{1}{\mathrm{~L}_{1}} & 0 & 0 & 0 \\
0 & \frac{\mathrm{M}}{\mathrm{L}_{2} \mathrm{C}_{2}} & 0 & \frac{1}{\mathrm{C}_{2}} \\
0 & 0 & \frac{1}{\mathrm{~L}_{2}} & 0
\end{array}\right]\left[\begin{array}{c}
\mathrm{V}_{1} \\
\mathrm{I}_{1} \\
\mathrm{~V}_{2} \\
\mathrm{I}_{2}
\end{array}\right]
$$


Detuning each cavity allows numerical calculations of $V_{i}$, which is related to $E_{i}$ by $\mathrm{V}_{\mathrm{i}}=-\int_{0}^{\mathrm{L}} \mathrm{E}_{\mathrm{i}} \mathrm{dl}$ for a given mode separation. Allowing the cavities to be detuned over a range of frequencies, a plot of field balance versus mode separation is developed which is shown in Fig. 3.12. It should be noted that the deviation of the equivalent circuit LC model and experimental data is due to machining errors in the cell-to-cell coupling iris. There is excellent agreement between the 2-D field solvers and the equivalent circuit model. The 3-D MAFIA [39] simulations indicate that the cell-tocell coupling is weaker than expected. Modeling a curved surface in 3-D with a finite difference code introduces a stair-step effect on all curved surfaces. This in effect decreases the size of the cell-to-cell iris, which manifests itself in a decrease in the cell-to-cell coupling. The mesh size could be significantly decreased, which would in principle decrease the stair-step effect and improve the calculation of the cell-to-cell coupling, with a corresponding increase in the run time of the eigen mode problem. It was decided not to pursue the use of MAFIA [39] in the calculation of the cell-tocell coupling since SUPERFISH [5] was in excellent agreement with our experimental data.

\subsection{Frequency Measurement}

The experimental data presented in this section was taken using an HP-8510C Network Analyzer, the frequency resolution of which was measured to be $100 \mathrm{~Hz}$ in CW mode. As we discussed in Section 3.4.1, due to the cell-to-cell coupling, the independent cell frequencies are split to become the $\pi$ and 0 -mode resonance frequencies. The critical issue is to have the $\pi$-mode fields balanced with the $\mathrm{f}_{\pi}=2856$ $\mathrm{MHz}$ under vacuum and at a nominal operating temperature of $55^{\circ} \mathrm{C}$. The operating temperature of $55^{\circ} \mathrm{C}$ was chosen because mentioned earlier, the 1.6 cell rf gun does not use water cooling to stabilize its temperature but instead uses resistive heating and the temperature difference between the gun body and its environment for cooling. The maximum environmental temperature at Brookhaven Accelerator Test Facility during the summer can reach $37^{\circ} \mathrm{C}$. 


\subsubsection{Independent Cell Frequencies}

To a certain extent terminology can blur the distinction between coupled cavity resonant frequencies and independent cell resonant frequencies. In the specific case of the 1.6 cell rf gun, there are two distinct sets of frequencies. These are the independent full and half cell resonant frequencies and the $\pi$-mode and 0-mode resonant frequencies. In the limiting case of zero coupling between the cells, the distinction between the coupled and un-coupled frequencies vanishes.

In principle, this difference in modal and independent cell frequencies can be understood by the following argument that models the tuning process of the 1.6 cell rf gun. Utilizing the tuners in the full cell we detune the full cell such that the cell-to-cell coupling induced frequency shift is negligible. This is the point where the modal frequencies can be identified with the independent resonant frequencies of the cavities. Using the full cell tuners we now scan the full cell frequency through the bandwidth of the tuners and at each data point we measure the modal and independent cell frequencies. To measure the full cell frequency we can detune the half cell, thereby decoupling the modal and full cell frequencies from the half cell. Note that the half cell frequency is unperturbed during the modal frequency measurements for each data point. We have modeled this experimental procedure using our equivalent circuit representation of the cell-to-cell coupling, the results of which are presented in Fig. 3.14.

The salient features of this plot are at the high and low end of the full cell's tuner range: the cell's independent frequencies can be associated with specific modal frequencies. Specifically, if the full cell is at the lower frequency end of its tuner range, the full cell independent frequency is the 0 -mode and the half cell independent frequency is the $\pi$-mode. Conversely, if the full cell is at the high frequency end of its tuner range, the full cell independent frequency is the $\pi$-mode and the half cell independent frequency is the 0-mode. When the cell's frequencies are equal, an avoided crossing occurs. This is the point of minimum mode separation and equal stored energy in both of the cavities.

Mode separation is used to balance the $\pi$-mode field in the 1.6 cell $\mathrm{rf}$ gun. Fig. 3.15 is the output of a HP-8510C Network Analyzer measuring the reflected 


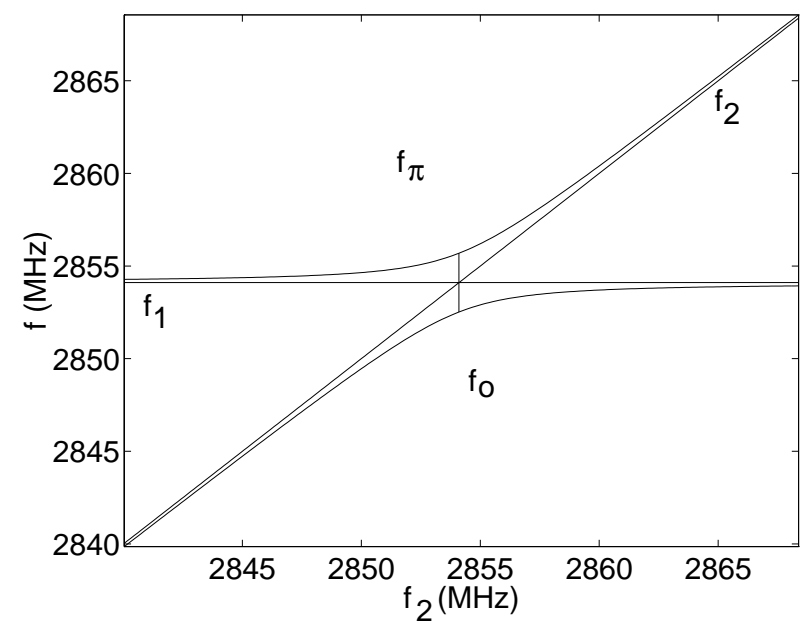

Figure 3.14: Model of modal and independent cell frequency.

signal, $\mathrm{S}_{11}$, for the $\pi$ - mode.

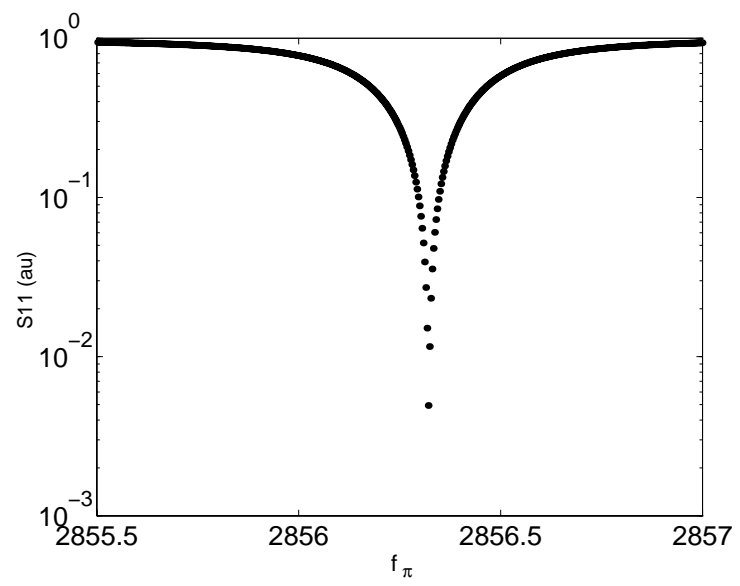

Figure 3.15: Network analyzer data for balanced fields.

\subsection{Longitudinal Field Measurements}

Using Slater perturbation theorem, Eq. 3.13, we probed the on-axis accelerating field of the 1.6 cell rf gun. Using a $4.72 \mathrm{~mm}$ spherical dielectric bead, of unknown dielectric constant, we can directly measure the relative field strength of the longitudinal accelerating field, $\left|\frac{E_{z}}{E_{z, \max }}\right|$, in the rf gun. 
The dielectric sphere was supported along the beam axis by a 4 pound test fishing line. Gravity was perpendicular to the fishing line while the longitudinal field measurements were being conducted. The fishing line was under maximum tension during these experiments. This removed much of the gravity-induced catenary of the sphere and its fishing line support. Two small holes, one in the cathode plate and the other on the beam exit port, constrained the radial position of the fishing line and hence the radial position of the perturbing sphere. This allowed the bead to be positioned at a given position in space while the rf gun was moved by a longitudinal bead pull cart. In this way, we were able to position the spherical bead at any $\mathrm{z}$ location in the rf gun.

The frequency shift was measured using a self-excited loop, see Fig 3.11, instead of a network analyzer, which increased the accuracy of the frequency resolution down to $100 \mathrm{~Hz}$. The experimental setup for this measurement is depicted in Fig. 3.16 and the experimental results are shown in Fig. 3.17. An image charge effect is clearly seen in the region of cathode, $\mathrm{z}=0 \mathrm{~cm}$.

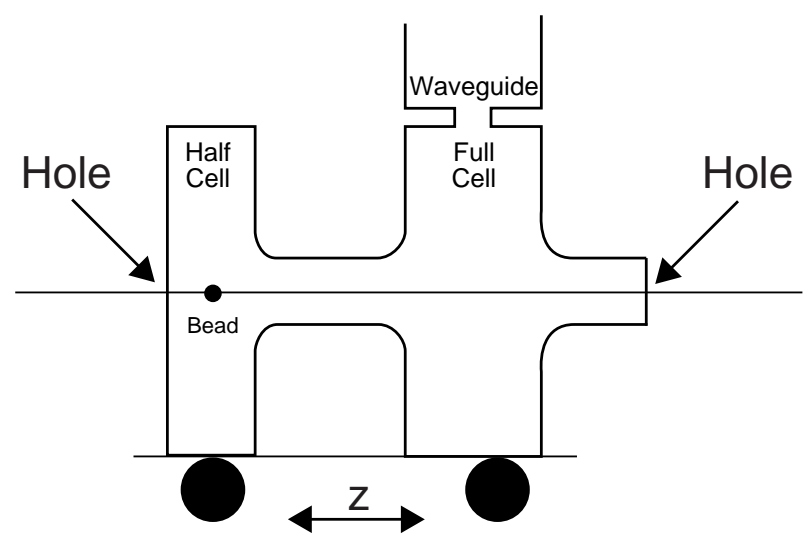

Figure 3.16: Bead pull experimental setup.

\subsection{Transverse Field Measurements}

In Section 3.3.1 we pointed out that the symmetrization of $\mathrm{E}_{\mathrm{z}}$ was one of the major design changes in the 1.6 cell rf gun. To investigate the effect of symmetrization 


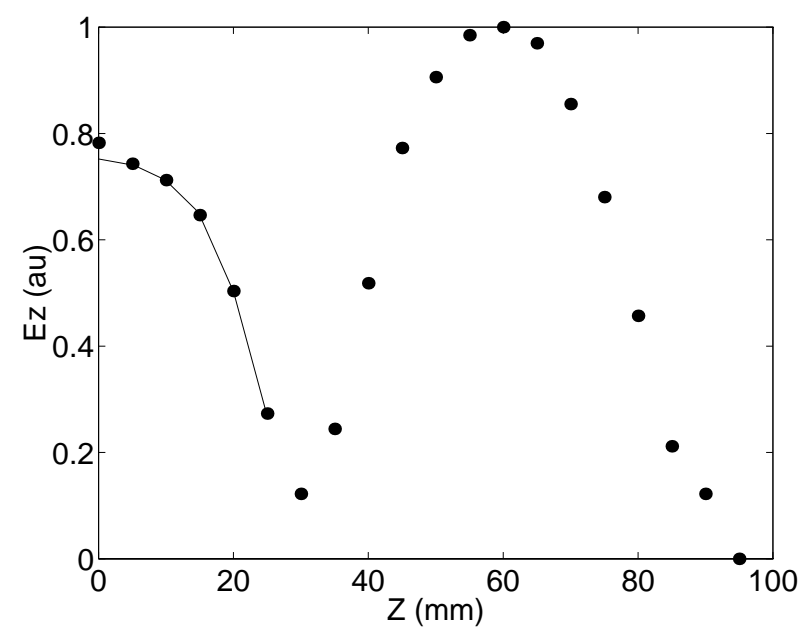

Figure 3.17: Longitudinal accelerating field measurement with the rf gun out of field balance.

we have conducted a series of field measurements using both disk pull and needle rotation techniques. Both techniques were used on each of two different rf coupling hole configurations to determine the optimum method of field symmetrization.

Fig. 3.18 depicts the two rf coupling hole schemes studied. Both coupling schemes utilize magnetic field coupling. Both coupling schemes were found to have the same dipole field contribution after symmetrization. The resistive coupling slot is much smaller in longitudinal direction and therefore has more structural strength than the capacitive coupling scheme. For this reason the resistive coupling slot was selected for use in the rf gun rather than capacitive coupling.

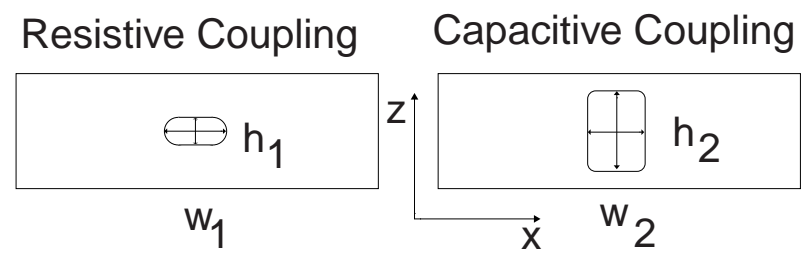

Figure 3.18: Resistive and capacitive rf coupling slots designs.

The resistive coupling slot in Fig. 3.18 has the following dimensions: $h_{1}=0.375^{\prime \prime}$ and $\mathrm{w}_{1}=0.829^{\prime \prime}$ with a minimum thickness of $\mathrm{T}_{1}=0.0917^{\prime \prime}$. The capacitive coupling slot in Fig. 3.18 has the following dimensions: $\mathrm{h}_{2}=0.913^{\prime \prime}$ and $\mathrm{w}_{2}=0.650^{\prime \prime}$ with a 
minimum thickness of $T_{2}=0.108^{\prime \prime}$.

\subsubsection{Transverse Disk Pulls}

Fig. 3.19 shows the experimental setup for our transverse disk pull which uses a $6.35 \mathrm{~mm}$ diameter $0.30 \mathrm{~mm}$ thick Copper $(\mathrm{Cu})$ disk to measure $\mathrm{E}_{\mathrm{z}}\left(\mathrm{x}_{0}, \mathrm{y}, \mathrm{z}_{\mathrm{o}}\right)$.

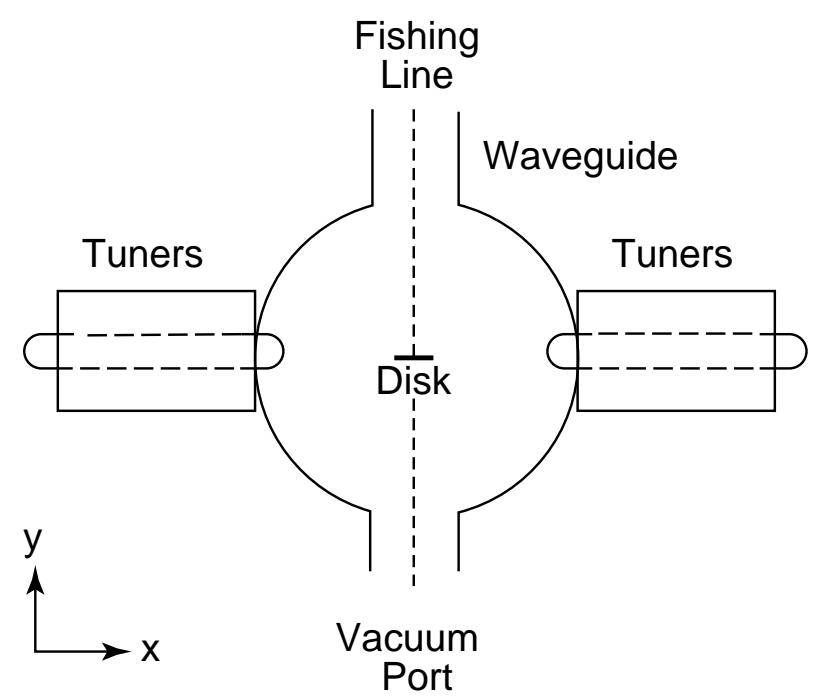

Figure 3.19: Experimental setups for full cell disk pull.

Experimental disk pull data is shown in Figs. 3.20 and 3.21 for the resistive and capacitive coupling scheme, respectively, for both before and after symmetrization.

The dipole offset of the $\mathrm{J}_{0}$ Bessel function, which models the radial dependence of the $\mathrm{E}_{\mathrm{z}}$ in a cylindrical symmetric accelerating cavity, is directly measured by this technique. Fitting the experimental data to Eq. 3.23 which is our mathematical model to second order for the $\mathrm{J}_{0}$ Bessel function, we have produced Table 3.4 which clearly shows that the dipole offset is reduced by more than an order of magnitude after symmetrization for both coupling schemes.

$$
\mathrm{E}_{\mathrm{z}}\left(\mathrm{x}=0, \mathrm{y}, \mathrm{z}_{\mathrm{o}}\right)=\mathrm{A}+\mathrm{B}(\mathrm{y}-\mathrm{C})^{2}
$$

Where $z_{\mathrm{o}}$ is the longitudinal position of the disk, which is in the middle of the full cell. $\mathrm{C}$ is the dipole offset in Table 3.4. 


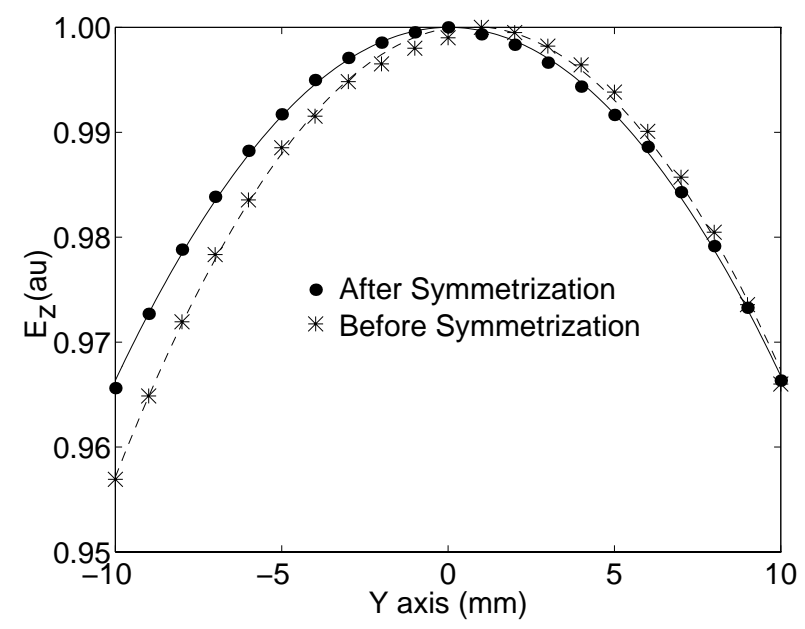

Figure 3.20: Longitudinal accelerating field as a function of transverse position for the resistive coupling case before and after symmetrization.

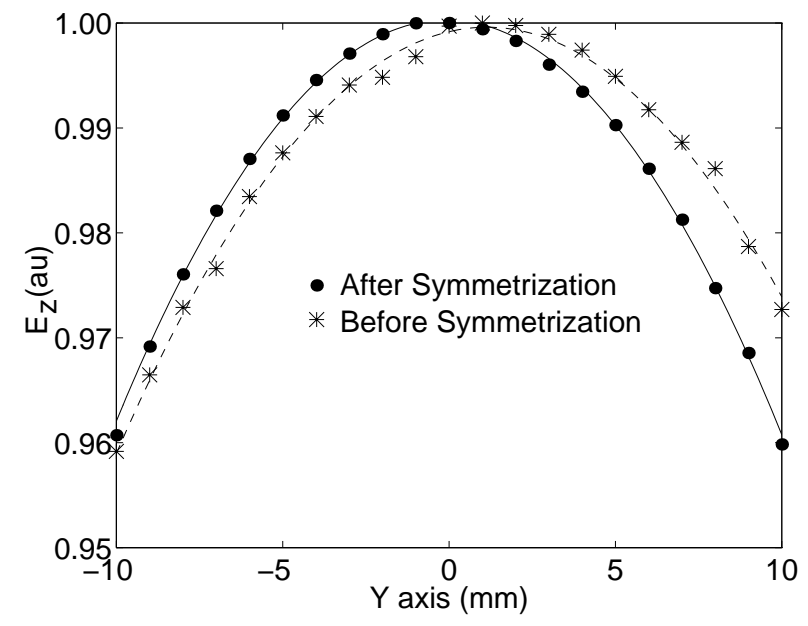

Figure 3.21: Longitudinal accelerating field as a function of transverse position for the capacitive coupling case before and after symmetrization.

\begin{tabular}{|c|c|c|}
\hline Coupling Mode & Symmetrization & Dipole Offset \\
\hline Resistive & Before & $+0.6760 \mathrm{~mm}$ \\
\hline Resistive & After & $+0.0303 \mathrm{~mm}$ \\
\hline Capacitive & Before & $+1.1395 \mathrm{~mm}$ \\
\hline Capacitive & After & $-0.0836 \mathrm{~mm}$ \\
\hline
\end{tabular}

Table 3.4: Dipole offset, C, before and after symmetrization for both resistive and capacitive coupling. 


\subsubsection{Needle Rotation Measurements}

In previous measurements of the dipole field in an $\mathrm{rf}$ cavity, needles were pulled longitudinally offset from the beam axis [40] or were pulled transversely [41] to the beam axis. In our needle rotation technique, a $1.27 \mathrm{~cm}$ long $0.635 \mathrm{~mm}$ diameter metal needle is positioned parallel to the beam axis and rotated in the $\Theta$ direction such that a cylinder with a radius of $\mathrm{R}=1.00 \mathrm{~cm}$ was swept out. Positioning the needle in the middle of the full cell, we only perturb $E_{z}$. The experimental setup used for the needle rotation can be seen in Fig. 3.22. Accurate positioning of the needle was accomplished by using a compound arrangement of optical translation and optical rotation stages. The translation stage allowed the positioning of the needle in the radial direction with respect to the beam pipe center. The rotation stage allowed the positioning of the needle in the $\Theta$ direction with respect to a fiducial mark that we defined as the x-axis.
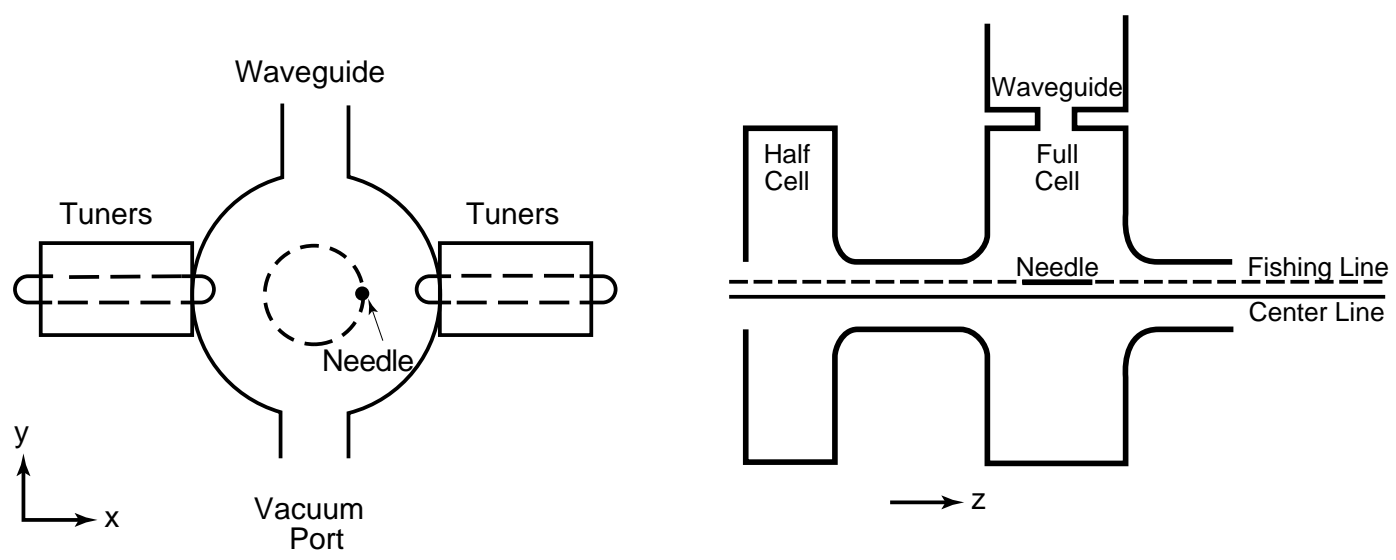

Figure 3.22: Transverse and longitudinal views of the needle rotation experimental setup.

Experimental needle rotation data is shown in Figs. 3.23 and 3.24 for the resistive and capacitive coupling schemes, respectively, for both before and after symmetrization. A cursory analysis of the coupling cases indicates that the dipole mode has been enhanced after symmetrization. This is true only in the tuner plane and is due to our inability to repeatedly reposition the single full cell tuner used during the low power rf testing of the 1.6 cell rf gun brass model. In these experiments, we were interested 
in the multi-pole mode contribution in the waveguide/vacuum port plane which on further analysis was found to be reduced.

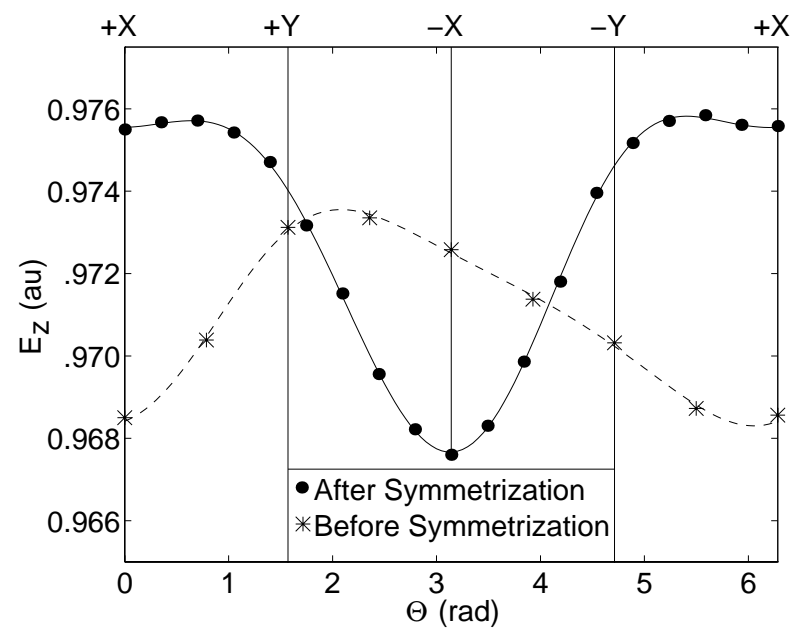

Figure 3.23: Longitudinal accelerating field as a function of $\Theta$ for the resistive coupling case before and after symmetrization.

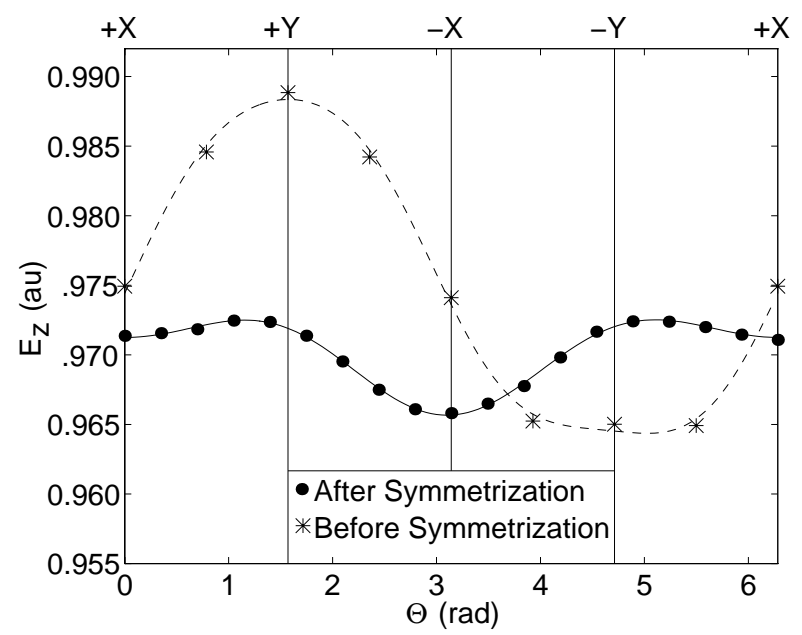

Figure 3.24: Longitudinal accelerating field as a function of $\Theta$ for the capacitive coupling case before and after symmetrization.

We have also produced 3-D field plots of the longitudinal accelerating field using this needle rotation technique. Positioning the needle at $\mathrm{R}=0.00 \mathrm{~cm}, \mathrm{R}=0.50 \mathrm{~cm}$, and $1.00 \mathrm{~cm}$ we swept out a cylinder, taking data every $45^{\circ}$. We have produced the 3-D $\mathrm{E}_{\mathrm{z}}$ field plots shown in Figs. 3.25 and 3.26, for both the resistive and capacitive 
coupling cases respectively, using this technique. Note that the 3-D field plots in Figs. 3.25 and 3.26 are before symmetrization.

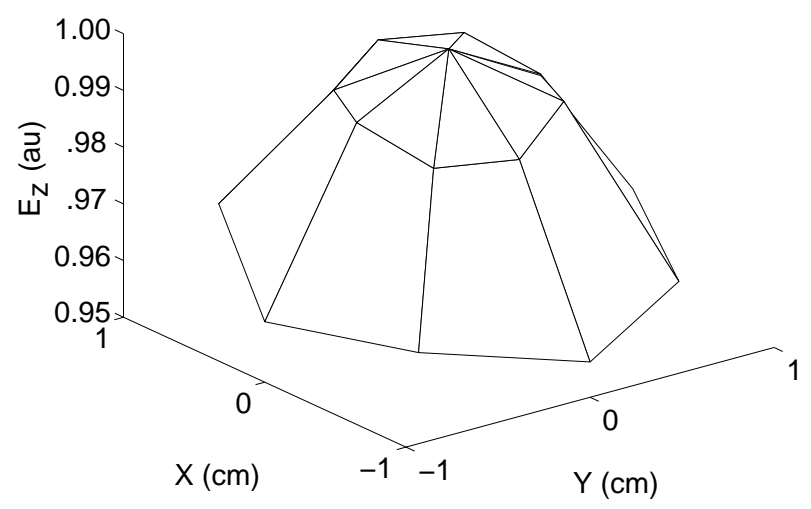

Figure 3.25: 3-D plot of the longitudinal accelerating field for the resistive coupling case, before symmetrization.

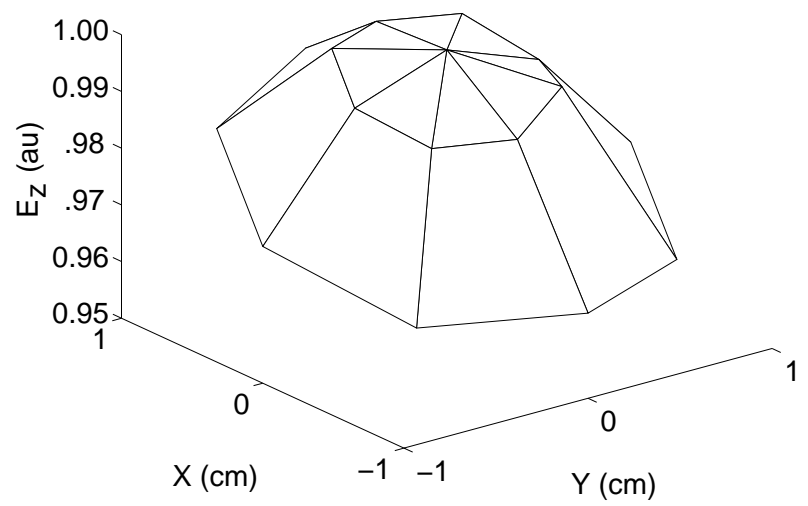

Figure 3.26: 3-D plot of the longitudinal accelerating field for the capacitive coupling case, before symmetrization.

\section{Multi-Pole Analysis}

Fitting the data in Figs 3.23 and 3.24 with a Fourier series, Eq. 3.24, it is possible to calculate the higher order mode contribution to the longitudinal accelerating field. 
The calculated Fourier series coefficients for the waveguide/vacuum port plane are presented in Figs. 3.27 and 3.28 for both resistive and capacitive coupling, before and after symmetrization.

$$
\mathrm{E}_{\mathrm{z}}=\sum_{\mathrm{m}=1}^{\infty} \mathrm{A}_{\mathrm{m} 10} \mathrm{TM}_{\mathrm{m} 10}
$$

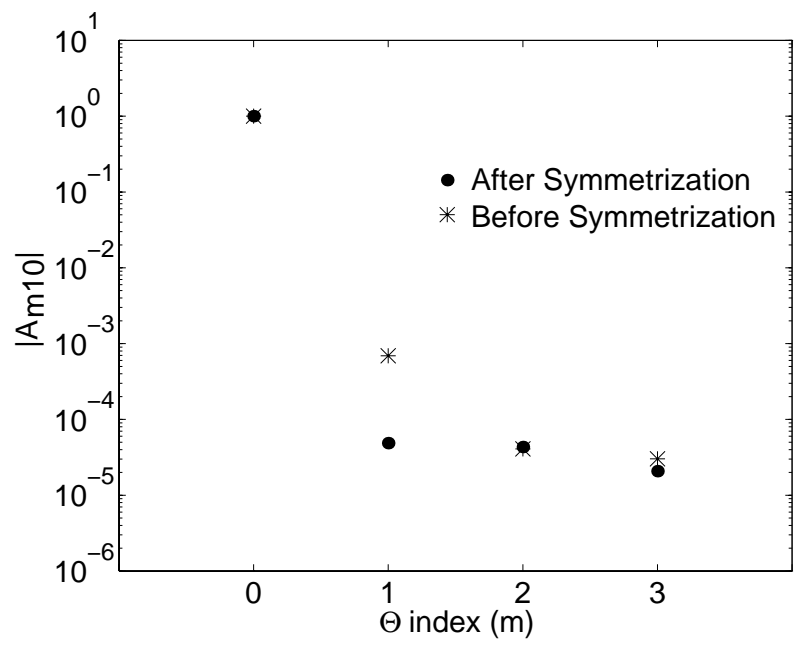

Figure 3.27: Fourier coefficient for the resistive coupling case before and after symmetrization.

The multi-pole coefficients up to the sextupole are presented in Table 3.5 for use in estimating the multi-pole contributions to the transverse emittance growth in Chapter 2, Section 2.3.4. The needle rotation data is consistent with the disk pull data in that the capacitive coupled rf dipole shift is greater than the resistive coupled rf case before symmetrization. Both coupling schemes' dipole contributions are approximately equal after symmetrization.

Since the two coupling schemes are approximately equal in their symmetrized dipole mode contribution, this effect was not utilized as the final selection criteria coupling slot design. The resistive coupled rf scheme was selected for the rf coupling scheme in the OFHC Cu 1.6 cell rf gun design, since its mechanical design is more structurally rigid that the capacitive coupling scheme. Also the tolerance on the symmetrizing hole is much looser for the resistive coupling scheme, since the asymmetry 


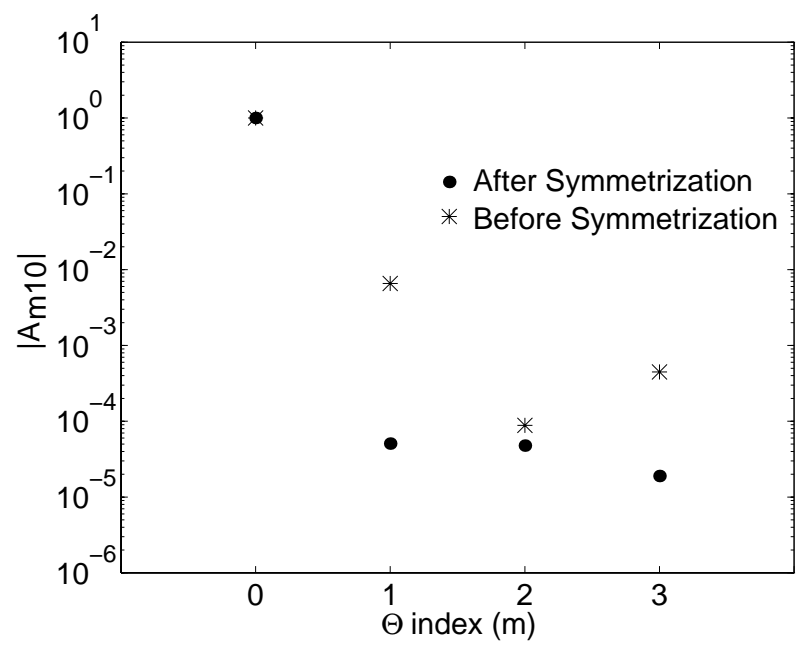

Figure 3.28: Fourier coefficient for the capacitive coupling case before and after symmetrization.

\begin{tabular}{|l|l|l|l|l|l|}
\hline Coupling Mode & Symmetrization & $\mathrm{m}=0$ & $\mathrm{~m}=1$ & $\mathrm{~m}=2$ & $\mathrm{~m}=3$ \\
\hline Resistive & Before & 1.000 & 0.0006918 & 0.0000408 & 0.0000302 \\
\hline Resistive & After & 1.000 & 0.0000487 & 0.0000433 & 0.0000208 \\
\hline Capacitive & Before & 1.000 & 0.0065562 & 0.0000885 & 0.0004472 \\
\hline Capacitive & After & 1.000 & 0.0000508 & 0.0000478 & 0.0000190 \\
\hline
\end{tabular}

Table 3.5: Multi-pole coefficients of the Fourier series before and after symmetrization for both resistive and capacitive coupling. 
being correct for is much smaller.

\subsection{Waveguide to $\pi$-mode Coupling}

\subsubsection{Bethe Hole Coupling Theory}

The final goal during manufacturing and tuning of the 1.6 cell $\mathrm{rf}$ gun is to have balanced fields for the $\pi$-mode at a resonant frequency of $2856 \mathrm{MHz}$ under vacuum at $55^{\circ}$ with the waveguide to $\pi$-mode over-coupled and equal to 1.05 . The frequency shift due from standard temperature, pressure, and humidity (STPH) to vacuum condition at $55^{\circ}$ is straightforward and will not be discussed here. However, to get balanced fields at $2856 \mathrm{MHz}$ with $\beta_{\mathrm{rf}}=1.05$ is more difficult, since the waveguide to full cell coupling hole effects both $\beta_{\mathrm{rf}}$ and the $\pi$-mode frequency.

We can use Bethe's theory of small hole aperture coupling [42] to calculate $\beta_{\mathrm{rf}}$. Experimentally, the coupling hole size can be found using the cut and measure technique. Then scaling laws [43] such as Eq. 3.25 can be used to calculate the change in coupling size to get the desired coupling parameter.

$$
\beta_{\mathrm{rf}}=\frac{\pi^{2} \mathrm{Z}_{\mathrm{o}} \mathrm{k}_{\mathrm{o}},{ }_{10} \mathrm{e}_{\mathrm{o}}^{4} \mathrm{l}_{1}^{6} \mathrm{e}^{-2 \alpha_{\mathrm{o}} \mathrm{d}}}{9 \mathrm{ab}\left[\mathrm{K}\left(\mathrm{e}_{\mathrm{o}}\right)-\mathrm{E}\left(\mathrm{e}_{\mathrm{o}}\right)\right]^{2}} \frac{\mathrm{H}_{1}^{2}}{\mathrm{P}_{\mathrm{o}}}
$$

where:

- $\mathrm{Z}_{\mathrm{o}}=120 \pi$ and is the impedance of free space;

- $\mathrm{k}_{\mathrm{o}}=\frac{2 \pi}{\lambda_{\mathrm{rf}}}$ where $\lambda$ is the wavelength in free space;

- ${ }_{10}=\mathrm{k}_{\mathrm{o}} \sqrt{1-\left(\frac{\lambda_{\mathrm{rf}}}{2 \mathrm{a}}\right)^{2}}$;

- $l_{1}$ and $l_{2}$ are the half major and minor axes of the coupling aperture;

- $\alpha_{\mathrm{o}}=\mathrm{k}_{\mathrm{o}} \sqrt{\left(\frac{\lambda_{\mathrm{rf}}}{\lambda_{\mathrm{c}}}\right)^{2}-1}$

- $\lambda_{\mathrm{c}}$ is the cutoff wavelength of the rf coupling iris;

- $\mathrm{a}$ and $\mathrm{b}$ are the width and height of the waveguide, where $\mathrm{a}>\mathrm{b}$;

- $\mathrm{K}\left(\mathrm{e}_{\mathrm{o}}\right)$ and $\mathrm{E}\left(\mathrm{e}_{\mathrm{o}}\right)$ are the complete elliptic integrals of the first and second kind, respectively;

- $\mathrm{H}_{1}$ is the magnetic field strength on the wall of the cavity if the aperture were replaced by a metal surface.; 
- $\mathrm{P}_{\mathrm{o}}$ is the total power loss in the cavity;

- $\mathrm{e}_{\mathrm{o}}=\sqrt{1-\left(\frac{1_{2}}{1_{1}}\right)^{2}}$;

- $\mathrm{d}$ is the coupling aperture thickness.

\subsubsection{Experimental Data}

Radio-frequency coupling measurements of the 1.6 cell $\mathrm{rf}$ gun after increasing the coupling iris size, using sinker electro discharge machining (EDM), showed that the design goal of $\beta_{\mathrm{rf}}=1.05$ over-coupled, was still not achieved. Fig. 3.29 is a Smith Chart representation of the impedance loci of the 1.6 cell $\mathrm{rf}$ gun after EDM. This clearly shows that the $\pi$-mode is under-coupled with $\beta_{\mathrm{rf}}=0.71$. Further increase in the coupling coefficient would have decreased the full cell independent resonant frequency by an unacceptable amount. An external matching section was used to increase $\beta_{\mathrm{rf}}$ without effecting the full cell independent resonant frequency. In principle, two types of correction schemes, capacitive or inductive matching section, could have been used. We chose to use the inductive matching scheme since the 1.6 cell rf gun

was designed to operate up to a peak field gradients of $150 \frac{\mathrm{MV}}{\mathrm{m}}$, as was discussed in Section 2.4.2 of Chapter 2. Inductive matching sections raise the peak electric field at the surface by a much smaller amount than capacitive matching.

\section{$\mathrm{Q}_{\mathrm{L}}$ Modification}

There are two issues that must be looked into to modify the $\pi$-mode coupling coefficient. First, how much inductive reactance must be added to the waveguide system to get the designed $\beta_{\mathrm{rf}}$ ? Second, where do we put the inductive post to produce the necessary reactance? These questions can be answered by the Smith Chart or we can use transmission line theory to calculate the necessary parameters.

The one set of curves that is not reproduced on the Smith Chart is lines of constant VSWR. These lines of constant VSWR are represented by circles with their centers located at the center of the Smith Chart. In our specific case the $\pi$-mode had a VSWR of 1.4; therefore, we can draw a circle whose radius emanates from $r=1$ on the resistive axis and ends at $\mathrm{r}=1.4$. This is represented in Fig. 3.29. The $\pi$-mode can take on 


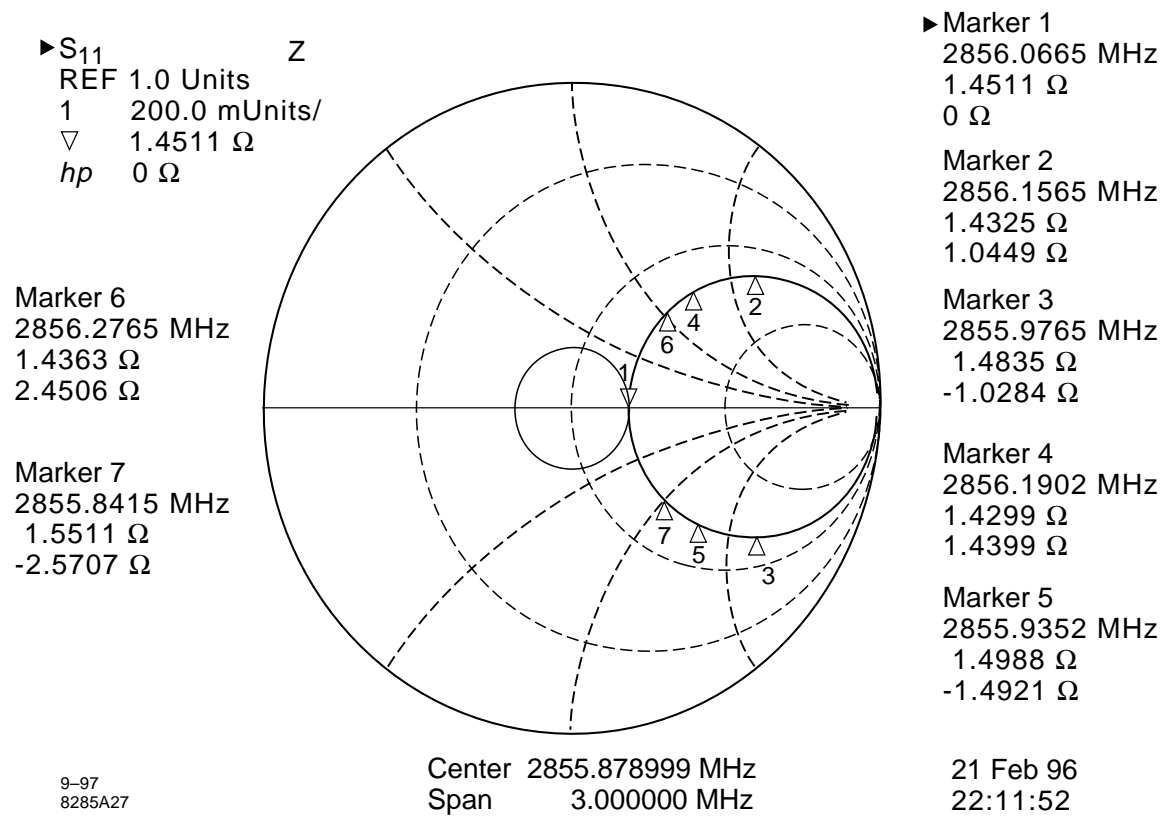

Figure 3.29: Under-coupled impedance locus.

any $\mathrm{Z}$ value on the constant VSWR circle. The design $\beta_{\mathrm{rf}}$ of 1.05 over-coupled can be attained where the VSWR circle intersects the circle of normalized resistance of $\mathrm{R}=$ 0.95. Graphically this will occur at the normalized reactance of $+\mathrm{i} 0.325$. Therefore, if we were to add the same amount but opposite sign of inductive reactance we would cancel the capacitive reactance at that point resulting in a resistive load of $\mathrm{R}=0.95$, which corresponds to the designed $\beta_{\text {rf }}$. So the first question has been answered. We need to add $+\mathrm{i} 0.325$ of normalized reactance to achieve the designed beta.

We can use Fig. 3.30 to graphically interpret the normalized transverse position of the inductive post in the waveguide to achieve the normalized reactance of 0.325 . An inductive post diameter of $0.125^{\prime \prime}$ was selected since after final brazing it might be necessary to perturb the post position. The result of the graphical interpretation of Fig. 3.30 is presented in Eq. 3.26. The normalizing parameter is given by the inner E wall dimension of a S-band waveguide, which is equal to $\mathrm{a}=2.840^{\prime \prime}$. 


\begin{tabular}{|l|l|l|l|}
\hline$\phi_{\mathrm{i}}$ & reference plane & detuned short plane & Total Length \\
\hline$\phi_{1}$ & 66.03325 & \pm 180 & \\
\hline$\phi_{2}$ & \pm 180 & 0.24495 & \\
\hline $\mathrm{L}$ & $0.34171 \lambda_{\mathrm{g}}$ & $0.25034 \lambda_{\mathrm{g}}$ & $0.59205 \lambda_{\mathrm{g}}$ \\
\hline
\end{tabular}

Table 3.6: Summary of shorting experiments.

$$
\begin{array}{ll}
\operatorname{Im}\{\mathrm{Z}\}= & +0.325 \\
\overline{\mathrm{R}}= & +0.022 \\
\overline{\mathrm{D}}= & +0.160
\end{array}
$$

Now we can answer the last question, where along the S-band waveguide do we locate the inductive post? We can use the pragmatic approach and use a spring loaded pin to experimentally investigate the coupling as a function of pin position. Another approach is to use the wavelength scale located on the outer diameter of the Smith Chart to measure where along the waveguide the inductive pin should be located. This position is not unique, but is $\frac{\mathrm{n} \lambda_{\mathrm{g}}}{2}$ periodic.

We use a two-port Transmission Reflection Load (TRL) calibration of our HP8510C Network Analyzer to find the reference plane and the plane of the detuned short. Measuring the phase of the $\mathrm{S}_{11}$ signal with a shorted waveguide located at the S-band launcher, we can calculate the position of the reference plane with respect to the launcher plane using Eq. 3.27.

$$
\phi_{2}=\phi_{1}-2 \beta \mathrm{L}
$$

where $\beta$ is the propagation constant of the structure and is equal to $\frac{2 \pi}{\lambda}$.

Now, detuning the rf gun we can find the plane of the detuned short by the same technique. These results are summarized in Table 3.6.

We can now calculate the longitudinal position of the inductive pin with respect to the plane of the detuned short using the transmission line equation, Eq. 3.28. Knowing the position of the plane of the detuned short is critical since it allows the experimenter to have a repeatable, known location without having to disassemble his experimental apparatus. 


\begin{tabular}{|l|l|}
\hline $\operatorname{Im}\{\mathrm{Z}\}$ & +0.325 \\
\hline$\overline{\mathrm{R}}$ & +0.022 \\
\hline $\mathrm{D}$ & +0.160 \\
\hline DetunedShort & $+0.59205 \lambda_{\mathrm{g}}$ \\
\hline $\mathrm{L}$ & $+0.122 \lambda_{\mathrm{g}}$ \\
\hline
\end{tabular}

Table 3.7: Parameters for position of the inductive post.

\begin{tabular}{|l|l|l|}
\hline Parameter & Before & After \\
\hline$\frac{\mathrm{E}_{2}}{\mathrm{E}_{1}}$ & 1.00 & 1.00 \\
\hline $\mathrm{f}_{\pi}$ & $2856.0665 \mathrm{MHz}$ & $2856.3175 \mathrm{MHz}$ \\
\hline Temperature & $19.0^{\circ} \mathrm{C}$ & $14.6^{\circ} \mathrm{C}$ \\
\hline $\mathrm{Q}_{\mathrm{o}}$ & 11200.3 & 11285.3 \\
\hline $\mathrm{Q}_{\mathrm{ext}}$ & 15867.0 & 11285.3 \\
\hline $\mathrm{Q}_{\mathrm{L}}$ & 6565.7 & 5607.2 \\
\hline$\beta_{\mathrm{rf}}$ & 0.71 & 1.01 \\
\hline$\delta \mathrm{f}$ & $3.25 \mathrm{MHz}$ & $3.25 \mathrm{MHz}$ \\
\hline
\end{tabular}

Table 3.8: Low power rf experimental results, before and after inductive post matching.

$$
\frac{\mathrm{Y}}{\mathrm{Y}_{\mathrm{o}}}=\frac{\sigma+\mathrm{i} \tan (\beta \mathrm{z})}{1+\mathrm{i} \sigma \tan (\beta \mathrm{z})}
$$

Solving Eq. 3.28 for the real part to be equal to $\frac{1}{1.05}$ we find the longitudinal position to place the inductive post. The external matching parameters are presented in Table 3.7. Since all of our measurements were conducted with an instrument to male Scarpaas flange spool piece, whose dimensions are important for the final pin tuning of the $\mathrm{OFHC} \mathrm{Cu}$ waveguide structure. These results are $\frac{\mathrm{n} \lambda_{\mathrm{g}}}{2}$ periodic.

The result of using the inductive post matching is shown in Fig. 3.31. The coupling constant is $\beta_{\mathrm{rf}, \pi}=1.01$ and is over-coupled. Comparison of our experimental coupling data, before and after inductive post matching, is tabulated in Table 3.8 


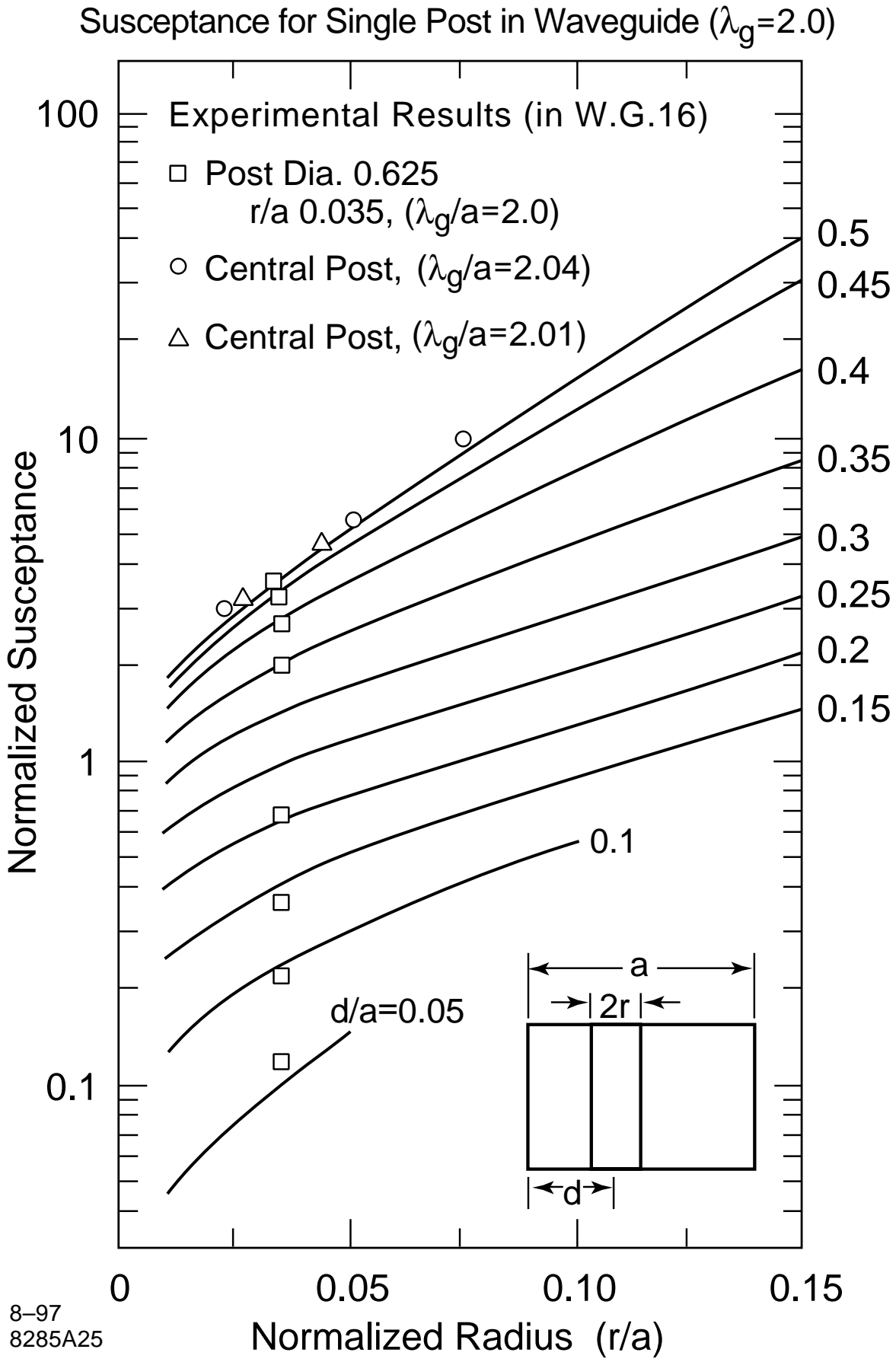

Figure 3.30: Data sheet used to match the waveguide to $\pi$-mode, for an inductive pin. 

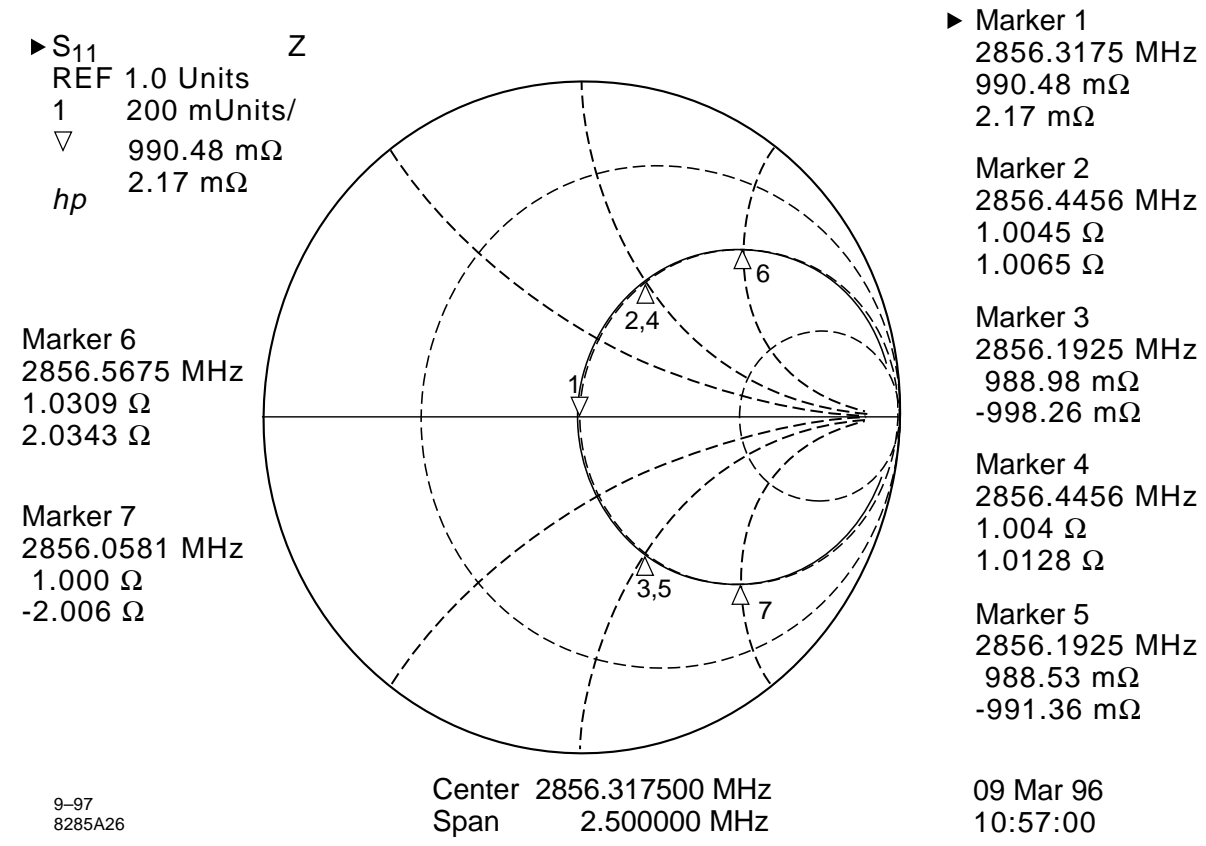

Figure 3.31: Impedance locus after inductive post matching.

\subsection{Conclusions}

In this Chapter we have presented microwave theory necessary to follow our low power rf measurements of the 1.6 cell rf gun. The rf gun design, manufacturing techniques and cleaning issues were also been addressed.

Electro-magnetic simulations of the rf gun were conducted, with SUPERFISH [5] and MAFIA [39], to insure the accuracy of our LC model for the rf gun field balance as a function of mode separation. Longitudinal field measurements of $\mathrm{E}_{\mathrm{z}}$ were also conducted to verify experimentally the LC model.

One of the major design features of 1.6 cell rf gun was the elimination of the $\mathrm{E}_{\mathrm{z}}$ dipole asymmetry in the middle of the full cell, and presumably through the whole rf gun. Measurements of $\mathrm{E}_{\mathrm{z}}$ as a function of the transverse coordinate $\mathrm{y}$ were conducted in the middle of the full cell using a transverse disk pull. Measuring the dipole offset of $\mathrm{E}_{\mathrm{z}}$ with the disk pull technique shows that for both the resistive and capacitive coupling schemes, there is over an order of magnitude decrease in the dipole offset 
between before and after symmetrization, as seen in Table 3.4.

Using our needle rotation technique we also studied $E_{z}$ as a function of $\Theta$, at a radius of $1 \mathrm{~mm}$ in the middle of the full cell. Using Fourier analyses on the needle rotation data, we were able to directly measure the higher-order mode contribution to $\mathrm{E}_{\mathrm{z}}$. We was over an order of magnitude decrease in the dipole contribution to $\mathrm{E}_{\mathrm{z}}$, for both resistive and capacitive coupling schemes.

From the comparison of Tables 3.4 and 3.5 we see that our needle rotation data is qualitatively consistent with the transverse disk pull data. We have been able to decrease the dipole mode contribution in the full cell by over an order of magnitude.

During the testing of the 1.6 cell rf gun it was apparent that the rf coupling constant, $\beta_{\mathrm{rf}}$, for the $\pi$-mode at $2856 \mathrm{MHz}$ and balanced fields was $\beta_{\mathrm{rf}}=0.54$ undercoupled. EDM was initially used to increase the rf coupling iris size and therefore $\beta_{\mathrm{rf}}$. This was moderately successful, attaining $\beta_{\mathrm{rf}}$ of 0.71 , but the full cell resonant frequency decreased by an unacceptable amount. An inductive post matching scheme was utilized to attain a rf coupling constant of $\beta_{\mathrm{rf}}=1.01$ without effecting the resonant frequency of the $\pi$-mode. 
Chapter 4

\section{Solenoidal Magnet}




\subsection{Introduction}

In the previous BNL emittance compensation magnet design, two identical air core solenoidal magnets are placed symmetrically around the cathode plane [44], see Fig. 4.1. Both magnets are supplied current from a single power supply. Upstream of the cathode plane is the bucking magnet with its current in the opposite sense of that of the downstream magnet, which is the emittance compensation magnet. With the current flow in the two identical magnets of the opposite sense, the magnet field at the cathode will in principle be zero. This configuration has the drawback that the field at the cathode is critically dependent on the mechanical alignment and construction of the two magnets with respect to the cathode plane.

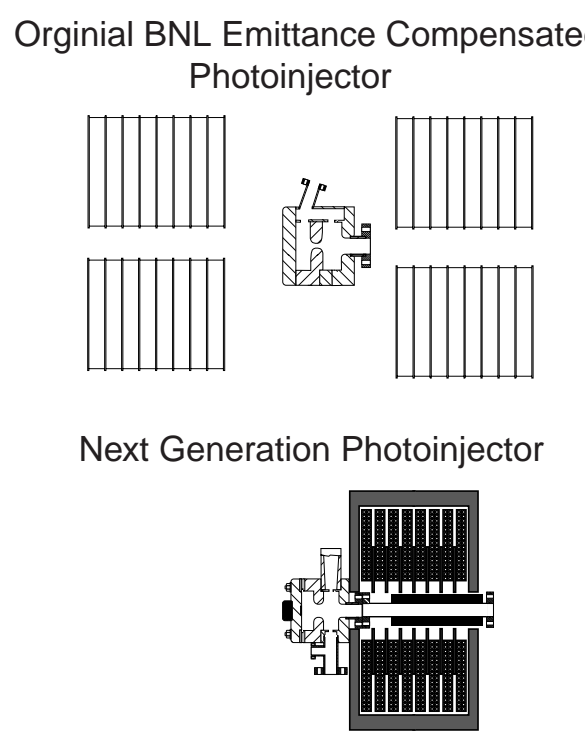

Figure 4.1: Drawing of original BNL/ATF air core emittance compensation magnet along with the single emittance compensation magnet. Both magnet designs are shown with their associated rf guns.

We have designed a single emittance compensation magnet, see Fig. 4.1, whose magnet field at the cathode has been minimized due to the use of magnetic pole faces and magnetic flux returns. The current is fed into the first magnet pancake from the port side. The current lead then exits the first pancake and loops over the solenoidal and is shielded by the 1006 magnetic steel so that the magnetic field from 
the lead does not affect the beam trajectory. The current lead then enters the second pancake from the starboard side. Alternating the current feed into the pancakes in this fashion eliminates the dipole field contribution on the beam axis due to the current pancake's internal wiring crossover. The magnet is magnetically symmetric in both the transverse and longitudinal dimensions.

\subsection{POISSON and PARMELA Simulation Program}

A simulation study was instituted using POISSON field maps in PARMELA [3]. To study the emittance compensation process as it relates to different solenoidal magnet designs, for each individual compensation magnet design a field map was produced for use in PARMELA [3]. Fig. 4.2 shows the 10 different solenoidal magnet designs that were investigated, labeled ECOMP0 thru ECOMP9. In Fig. 4.2, each sub-figure represents the mechanical design of the compensation magnet in the $\mathrm{R}$ $\mathrm{Z}$ plane. These sub-plots all have azimuthal symmetry. Fig. 4.3 consists of the $\mathrm{B}_{\mathrm{z}}$ field maps associated with their individual mechanical designs. These field maps were generated with a Dirichlet boundary condition at the cathode plane, thereby modeling a bucking/compensation magnet pair. This technique decreased the total number of mesh points and increased the speed of the calculations.

During these simulation studies a correlation between peak magnetic field position and minimum emittance achievable was noted. To eliminate the possibility that this correlation was an artifact of the magnet design, a series of POISSON runs were conducted using the ECOMP1 magnet design but varying the overall magnet position with respect to the cathode plane.

Using ECOMP1 mechanical designs but varying its position with respect to the cathode plane, field maps were generated and mapped into PARMELA [3]. A series of optimizing runs were conducted for each field map/magnet position. In Fig. 4.4, the minimum $\epsilon_{\mathrm{n}, \mathrm{rms}}$ attained for each magnet position is plotted versus the position of the magnetic field peak. This shows that there is an optimum magnet field position for 


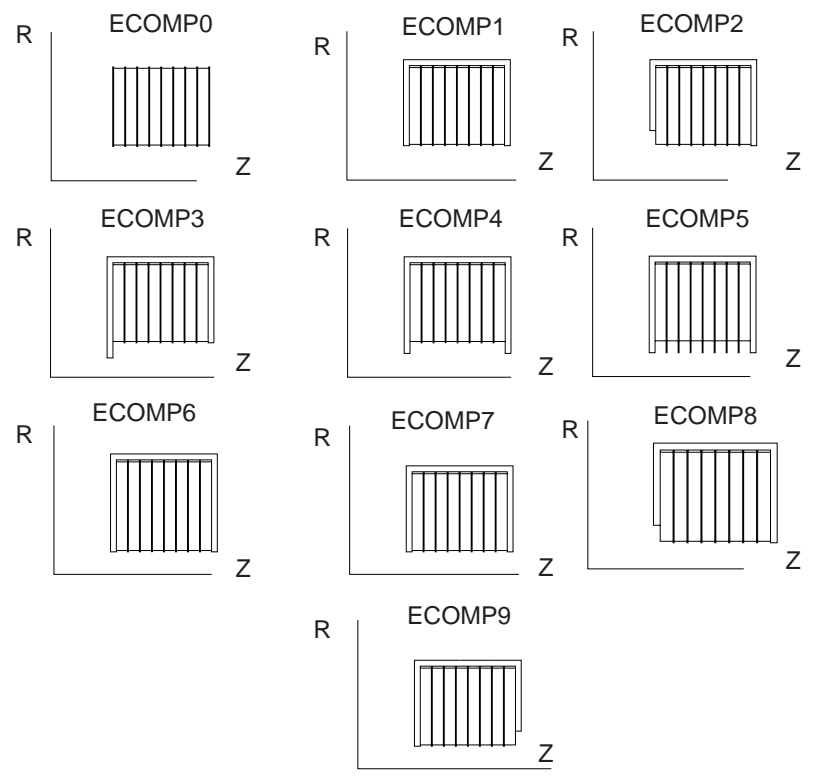

Figure 4.2: Magnet designs investigated using PARMELA.
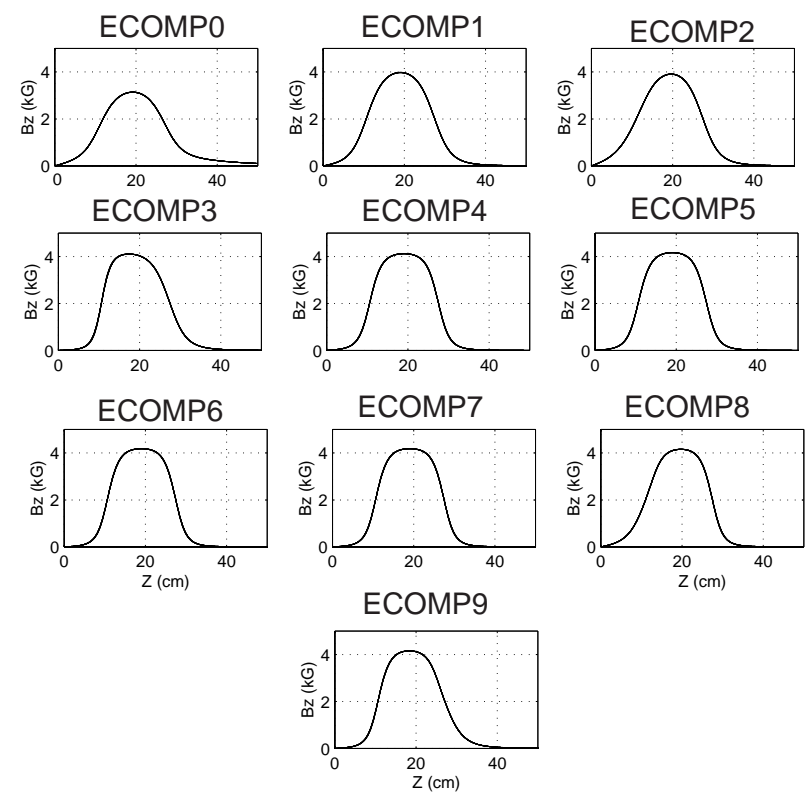

Figure 4.3: Longitudinal magnetic field profile of the magnet designs in Fig. 4.2. 
the emittance compensation process. This magnet field position cannot be attained in the BNL/SLAC/UCLA 1.6 cell S-band photoinjector due to mechanical constraints of the rf waveguide feed into the rf gun, see Fig. 4.1.

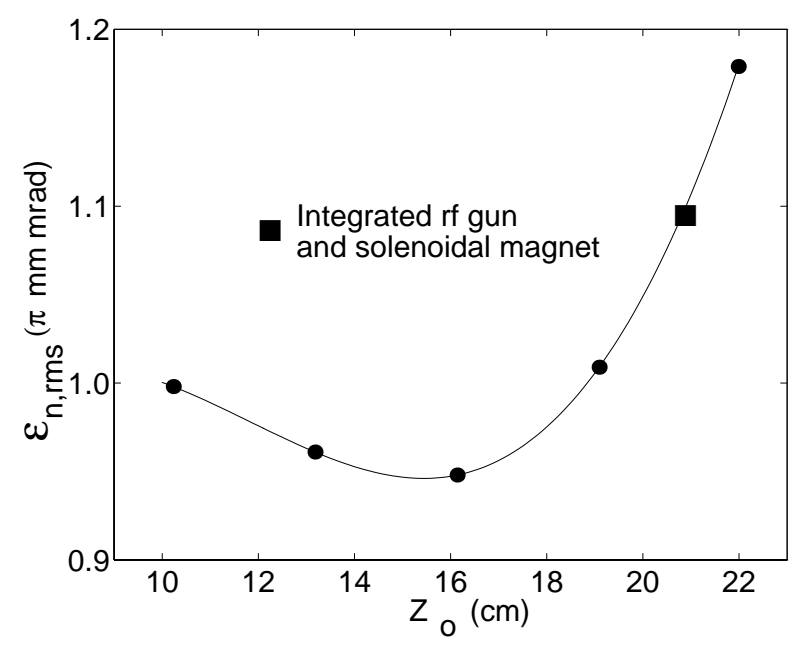

Figure 4.4: PARMELA simulation results of $\epsilon_{\mathrm{n}, \mathrm{rms}}$ versus ECOMP1 magnet position.

Major mechanical and rf changes would allow the BNL/SLAC/UCLA 1.6 cell Sband photoinjector to operate at the minimum of the $\epsilon_{\mathrm{n}, \mathrm{rms}}$ versus peak magnetic field position. This would allow for a $15 \%$ improvement in the emittance compensation process over the present photoinjector design. Due to budget and time constraints, it was decided not to pursue the mechanical and rf changes necessary to reposition the emittance compensation magnet to the optimum position indicated in Fig. 4.4.

\subsubsection{Production Magnet Design}

Due to the inability to position the ECOMP1 design at the optimum emittance compensation position without a major redesign of the rf gun waveguide feed, the POISSON file for the ECOMP1 magnet design was modified to position the magnet as close as possible to the gun. Using a larger boundary area upstream of the cathode to model the single compensation magnet design, the $\mathrm{B}_{\mathrm{z}}$ field at the cathode was of sufficient strength to cause unacceptable emittance growth.

The compensation magnet design ECOMP5 was selected for study, since its pole 
tips extend closer to the beam axis, thereby decreasing the $B_{z}$ field at the cathode. The cathode magnetic field, $\mathrm{B}_{\mathrm{z}_{\mathrm{o}}}$, of the ECOMP5 magnet design was sufficiently depressed to pursue its use as a single emittance compensation magnet.

Mid-plane axis

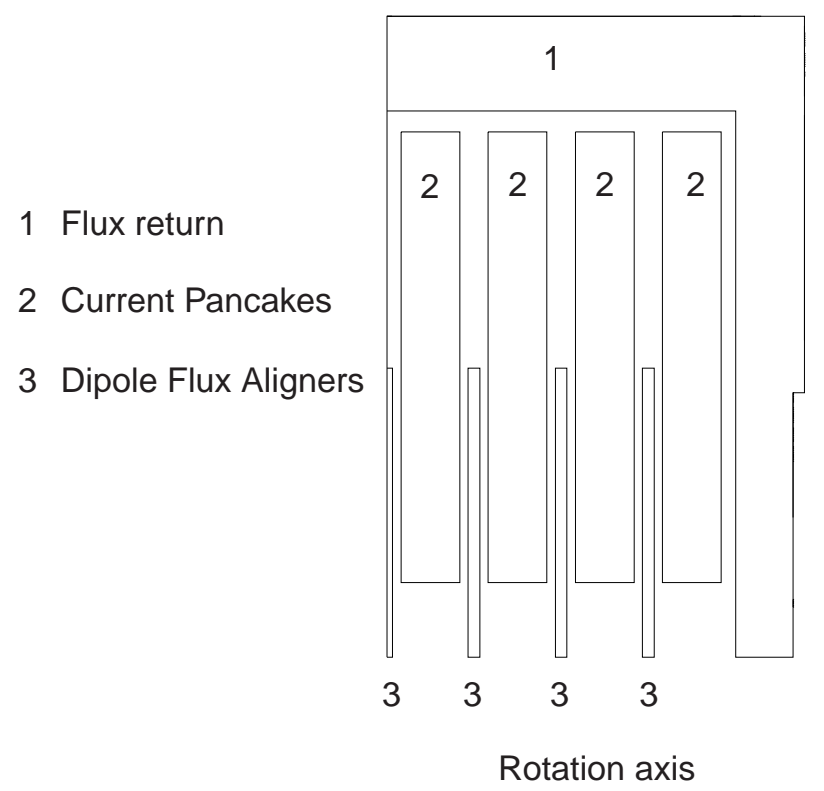

Figure 4.5: Final POISSON model of the single emittance compensation magnet.

The ECOMP5 design was further modified to insure that the 1006 steel flux returns and flux aligners would not saturate when the magnet was operated at the maximum current load of $200 \mathrm{~A}$. The single emittance compensation magnet design chosen for production is shown in Fig. 4.5. The magnet has two symmetries: axial symmetry around the beam line and reflection symmetry about the mid-plane of the magnet itself. Therefore it was sufficient to model only half of the magnet in the longitudinal direction with a Neumann boundary condition at the mid-plane symmetry plane. Modeling the magnet in this fashion decreased the time needed for calculation while maintaining the accuracy of the calculation, which was particularly important for studies of saturation effects of the 1010 steel. 


\subsection{Solenoidal Magnet Design}

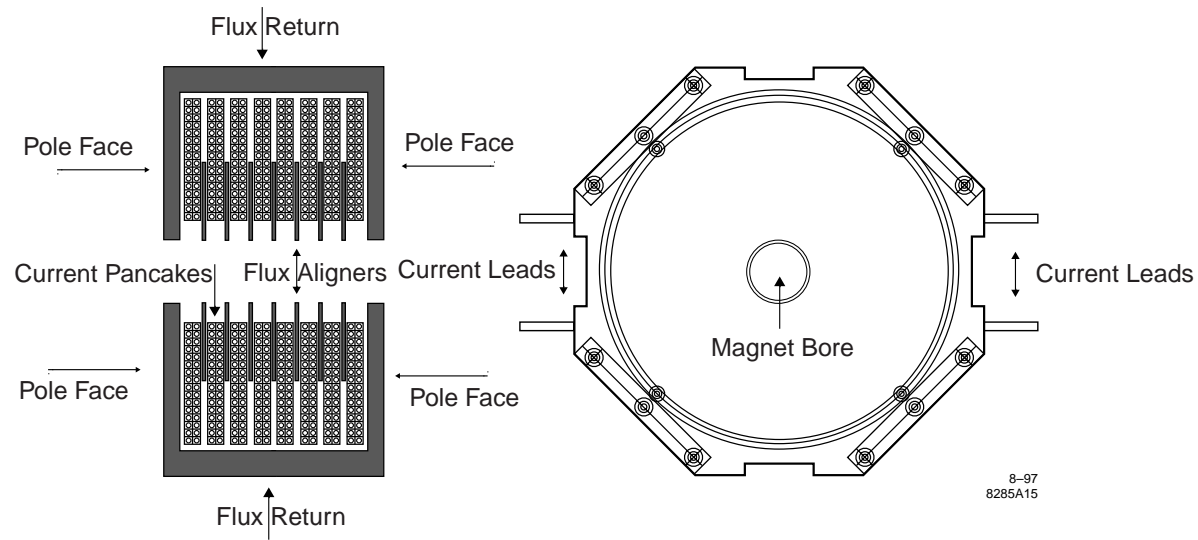

Figure 4.6: Solenoidal magnet mechanical drawing.

The solenoidal magnet is constructed out of eight double layer hollow core copper conductor pancakes. Table 4.1 contains the electric parameters of the emittance compensation magnet. Both the flux return and the seven dipole field flux aligners [45] were manufactured out of 1006 steel. These dipole field flux aligners are used to eliminate any dipole fields that arise from the current pancake asymmetries and misalignments during assembly. Transverse penetrations through the flux return for the hollow core current conductors were designed to have four-fold symmetry. This is believed to be an acceptably small deviation from axial symmetry. The external current conductors are fed to alternate sides of the solenoid to eliminate a dipole contribution due to the individual pancake conductor crossovers. A mechanical drawing of the solenoidal magnet is show in Fig. 4.6. The use of the magnetic flux returns and pole faces decreased the cathode magnetic field to $\mathrm{B}_{\mathrm{z}}(\mathrm{z}=0) \lesssim 10 \mathrm{G}$ for a peak magnetic field of $B_{z, \max }=3 \mathrm{kG}$, as seen in Fig. 4.7.

An air core bucking magnet is located at the upstream end of the rf gun. It was used to compare our theoretical predications and experimental results of emittance growth due to the cathode's finite magnetic field. These experimental results are presented in Section 5.9. A $\cos (\theta)$ deflection magnet is located in the bore of the emittance compensation magnet. This deflection magnet is used for energy measurements of the electron bunch energy at the exit of the gun. 


\begin{tabular}{|c|c|}
\hline Magnetic Material & 1006 Steel \\
\hline Conductor & Hollow \\
\hline Length & $200 \mathrm{~m}$ \\
\hline Cross Sectional Area & $0.39477 \mathrm{~cm}^{2}$ \\
\hline Resistance & $0.08613 \Omega$ \\
\hline Voltage & $18.95 \mathrm{~V}$ \\
\hline Power & $3445 \mathrm{~W}$ \\
\hline Current & $220 \mathrm{~A}$ \\
\hline $\mathbf{J}$ & $557.3 \frac{\mathrm{A}}{\mathrm{cm}^{2}}$ \\
\hline
\end{tabular}

Table 4.1: Emittance compensation solenoidal magnet mechanical and electric parameters.

\subsubsection{Field Measurements}

A major feature of the single emittance compensation magnet is the small cathode magnetic field, $B_{z}(z=0)$. A series of magnetic field measurements were conducted at the National Synchrotron Light Source Magnet Laboratory to insure that the cathode magnetic field, $\mathrm{B}_{\mathrm{z}}(\mathrm{z}=0)$, was within acceptable limits. Fig. 4.7 represents the longitudinal magnetic field produced by the emittance compensation magnet for the following excitation currents: 20, 50, 100, 150, and 200 Amperes. The rf gun's cathode plane is located $-21.5 \mathrm{~cm}$ from the center of the magnet, as indicated in Fig. 4.7.

The excitation plot of peak magnetic field versus excitation current is shown in Fig. 4.8. A least squares fit to these data is represented by Eq. 4.1.

$$
\mathrm{B}_{z, \max }=0.01423 \frac{\mathrm{kG}}{\mathrm{A}} \mathrm{I}+0.0007 \mathrm{kG}
$$

In Fig. 4.9 POISSON simulations are compared with the magnetic measurements of the single emittance compensation magnet. There is a $3.0 \%$ error between the POISSON simulation and the experimental results. This error could be due to the difference between 1010 steel used in the POISSON simulations and 1006 magnetic steel used in the manufacture of the compensation magnet.

The off axis longitudinal magnetic field was also measured. Fig. 4.10 represents the difference in $B_{z}(R=+5 \mathrm{~mm})$ and $B_{z}(R=0 \mathrm{~mm})$ for an excitation current of 150 


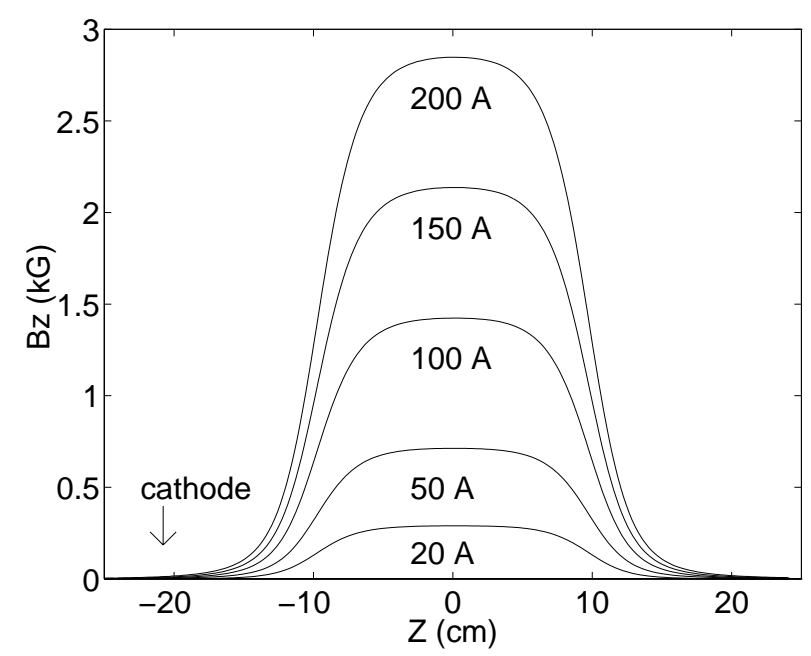

Figure 4.7: Magnetic field measurements for five different excitation currents.

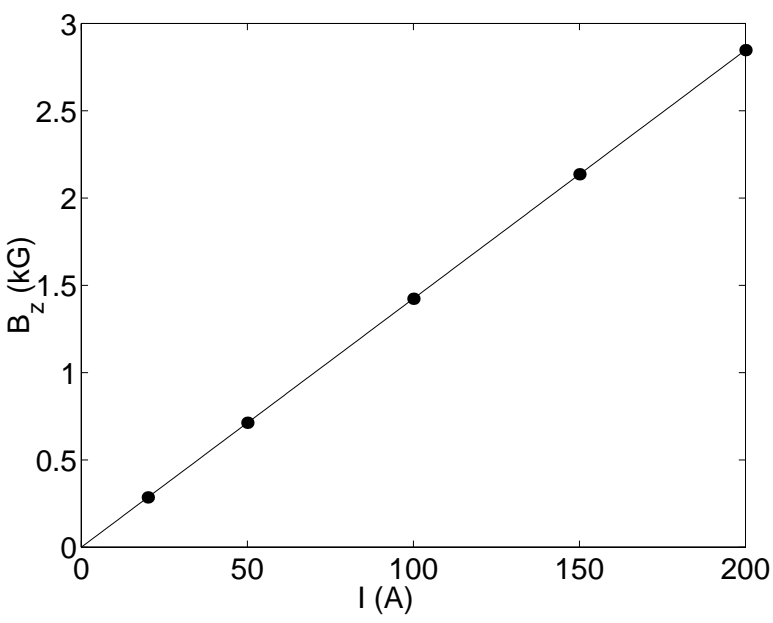

Figure 4.8: Peak magnetic field versus excitation current. 


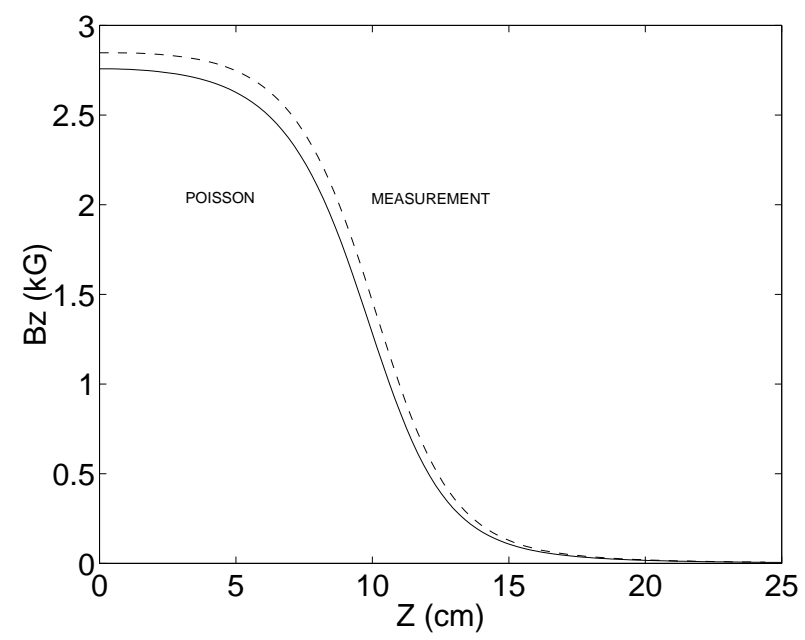

Figure 4.9: Magnetic field measurements.

Amperes. The maximum difference is less than $0.5 \%$.

The cathode $\mathrm{B}_{\mathrm{z}}$ field as a function of peak longitudinal magnetic field is shown in Fig. 4.11. The least squares fit to the data is presented in Eq. 4.2. The residual field of $0.29 \mathrm{G}$ in Eq. 4.2 is consistent with the earth's magnetic field. POISSON simulations indicate that the cathode field scales as $4.17 \frac{\mathrm{G}}{\mathrm{kG}}$ compared to $3.74 \frac{\mathrm{G}}{\mathrm{kG}}$ from magnet measurements.

$$
\mathrm{B}_{\mathrm{z}}(\mathrm{r}=0, \mathrm{z}=0)=3.74 \frac{\mathrm{G}}{\mathrm{kG}} \mathrm{B}_{\mathrm{z}, \max }+0.29 \mathrm{G}
$$

Using Eq. 4.3 for the definition of the effective magnetic length of the compensation magnet, we find that the magnetic length of the magnet is $\mathrm{L}_{\mathrm{eff}}=19.51 \pm 0.05 \mathrm{~cm}$ compared to $19.67 \mathrm{~cm}$ from our numerical analysis of the POISSON simulation data. POISSON simulation indicated that the solenoidal magnet would not go into saturation within the normal operating parameter of the magnet. The magnet length data prove that this is correct. If the magnet had gone into saturation at the higher current settings the magnet length would have increased as a function of excitation current. This was not the case, as can be seen clearly in Fig. 4.12.

$$
\mathrm{L}_{\mathrm{eff}}=\frac{1}{\mathrm{~B}_{z, \max }} \int_{-\infty}^{+\infty} \mathrm{B} \mathrm{d} \mathbf{l}
$$




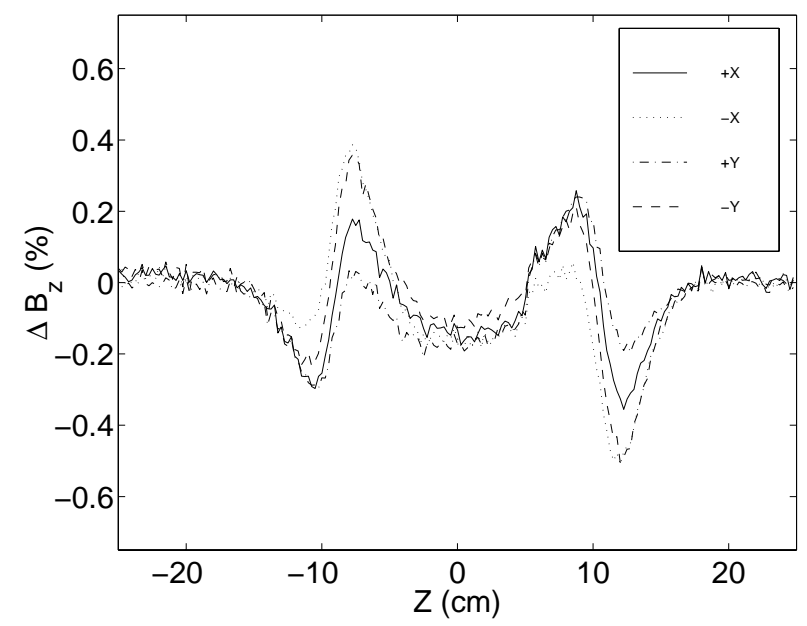

Figure 4.10: Difference in the off axis $(5 \mathrm{~mm})$ and on axis magnetic field measurement at 150 Amperes excitation current.

\subsection{Bucking Magnet Design}

An air core bucking magnet is located at the upstream end of the gun. It is comprised of 150 turns of 12 American Wire Gauge (AWG) copper (Cu) wire. The inner diameter (ID) is $140.00 \mathrm{~mm}$, the outer diameter (OD) of $185.53 \mathrm{~mm}$, and it is $28.04 \mathrm{~mm}$ long. The 12 AWG wire has a maximum rating of 25 Amperes.

The bucking magnet is only used for emittance growth studies due to cathode magnetic field. During experimental beam studies of emittance growth due to the cathode's finite magnetic field, the bucking magnet is supplied current by a uni-polar power supply. A precision shunt resistor was used to measure the current supplied to the bucking magnet. The cathode plane is located $3.625 \mathrm{~cm}$ downstream from the center of the bucking magnet.

\subsubsection{Field Measurements}

Fig. 4.13 represents experimental data of the longitudinal magnetic field, $\mathrm{B}_{\mathrm{z}}$ as a function of $\mathrm{z}$ due to air core bucking magnetic, for 5 and 10 Amperes of excitation current. The 10 Amperes magnetic field measurement data in Fig. 4.13 were fit to 


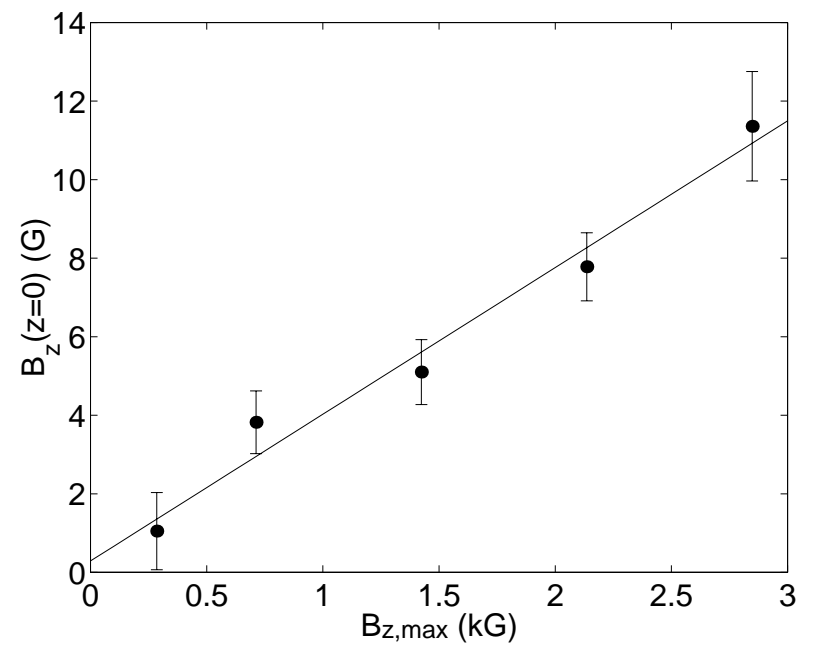

Figure 4.11: Cathode magnetic field versus peak magnetic field.

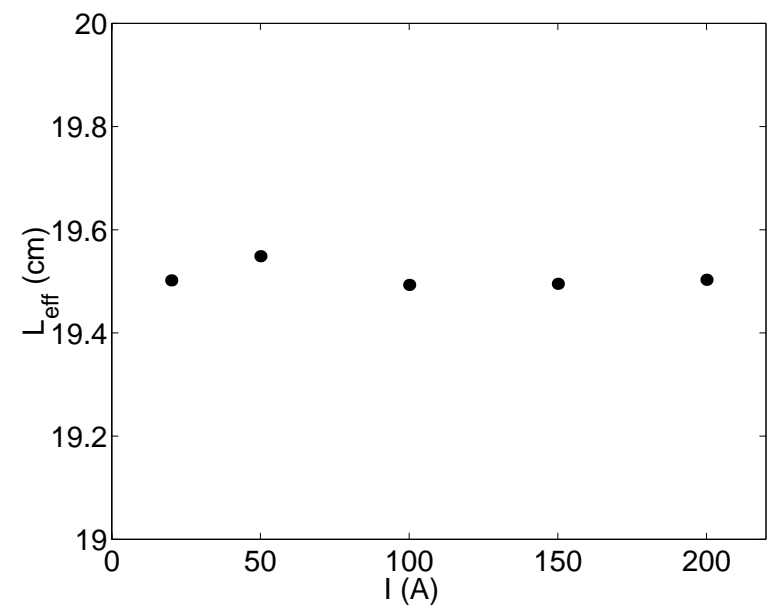

Figure 4.12: Effective magnetic length as a function of excitation current. 
Eq. 4.4. This fit models a single current loop located at a radial position, R, located at the center of the bucking magnet. The numerical model of the bucking and compensation magnet, allows us to predict the variation in the longitudinal magnetic field at the cathode plane for our studies of emittance growth due to the finite magnetic field at the cathode in Section 5.9.

$$
\mathrm{B}_{\mathrm{z}, \mathrm{BM}}(\mathrm{r}=0, \mathrm{z})=\frac{\mathrm{I} \mathrm{B}_{\mathrm{z}_{\mathrm{o}}}}{\left(1+\mathrm{A} \mathrm{z}^{2}\right)^{\frac{3}{2}}},
$$

where $\mathrm{I}$ is the current flowing through the bucking magnet in Amperes, $\mathrm{B}_{\mathrm{z}_{\mathrm{o}}}=11.60576 \frac{\mathrm{G}}{\mathrm{A}}$, and $\mathrm{A}=\frac{1}{\mathrm{R}^{2}}=0.0145 \frac{1}{\mathrm{~cm}^{2}}$. Using Eq. 4.4 the on axis magnetic field at the cathode scales like $\frac{\mathrm{dB}_{\mathrm{z}}}{\mathrm{dI}}=8.934 \frac{\mathrm{G}}{\mathrm{A}}$.

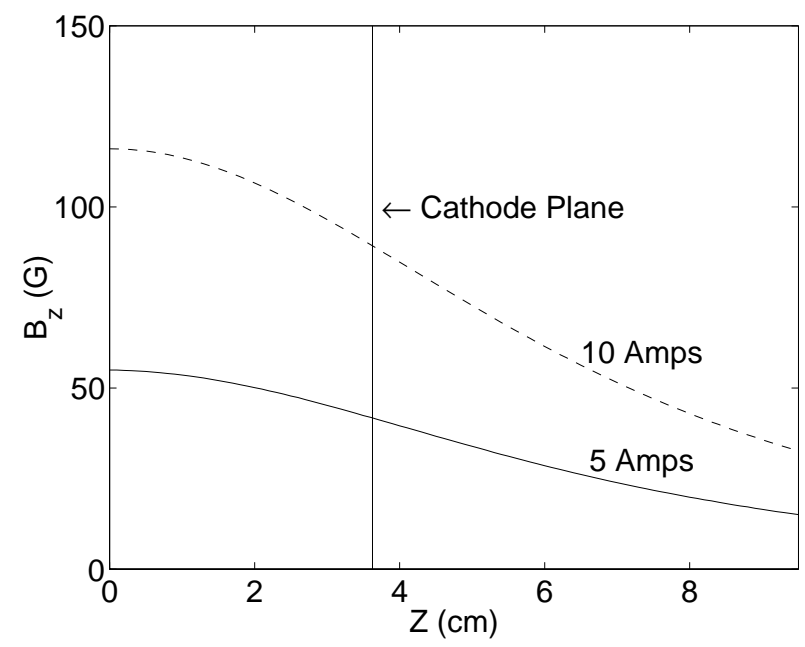

Figure 4.13: Bucking magnet field measurements for 5 and 10 Amperes.

\section{5 $\cos (\theta)$ deflection magnet}

A $\cos (\theta)$ deflection magnet is located in the bore of the emittance compensation solenoidal. This magnet is used for energy measurements of the electron bunch at the exit of the gun. The betatron rotation of the beam was taken into account in the energy analysis presented in Section 5.5.1. 


\subsubsection{Field Measurements}

$\int \mathbf{B} d \mathbf{l}$ measurements were accomplished while the deflection coil was positioned in the solenoidal magnet. This was critical since the flux straighteners enhance the dipole field of the deflection magnet. Fig. 4.14 represents the transverse magnetic field $B_{x}$ as a function of longitudinal position down the bore of the deflection magnet. The effective length of the steering magnet was measured to be $\mathrm{L}_{\mathrm{eff}}=11.32 \mathrm{~cm}$.

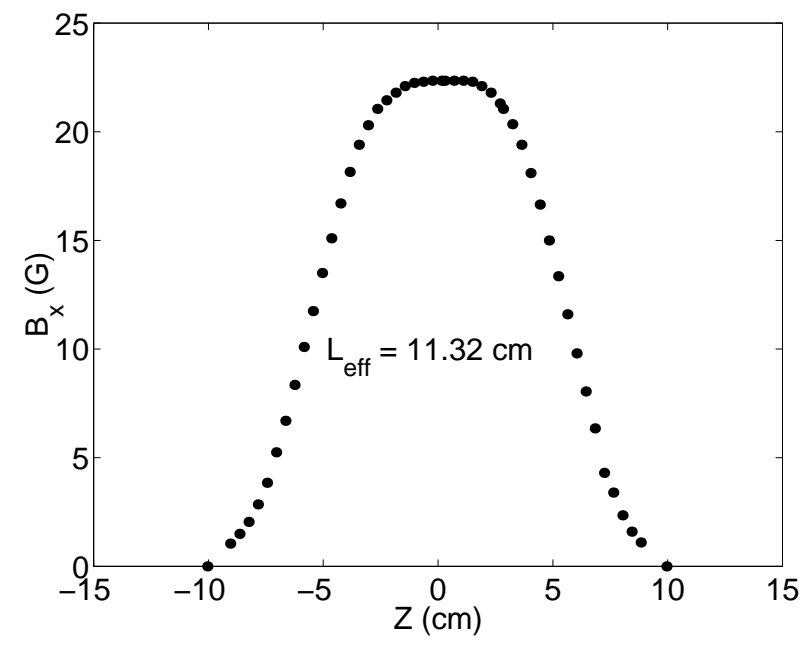

Figure 4.14: Transverse magnetic field profile.

As an aside, we shall prove that the transverse magnetic field in a long $\cos (\theta)$ deflection magnet is uniform. Using Amperes Law [46] Eq. 4.5, we can find the magnet field inside the $\cos (\theta)$ magnet in the following manner.

$$
\int \mathbf{B} \cdot \mathbf{d} \mathbf{l}=\frac{4 \pi}{\mathrm{c}} \mathrm{I}_{\text {enclosed }}
$$

Let us use the integration path shown in Fig. 4.15, where we intersect the $\cos (\theta)$ current sheet at $\pm \theta_{o}$.

$$
\int \mathbf{B} \cdot \mathbf{d} \mathbf{l}=\frac{8 \pi}{\mathrm{c}} \mathrm{I} \int_{0}^{\theta_{\mathrm{o}}} \cos (\theta) \mathrm{d} \theta
$$

Using the definition of the $\cos \left(\theta_{o}\right)$ we see that the integration length $\mathrm{y}_{\mathrm{o}}$ cancels out on both sides of the equality. This results in Eq. 4.7, that the transverse magnetic 


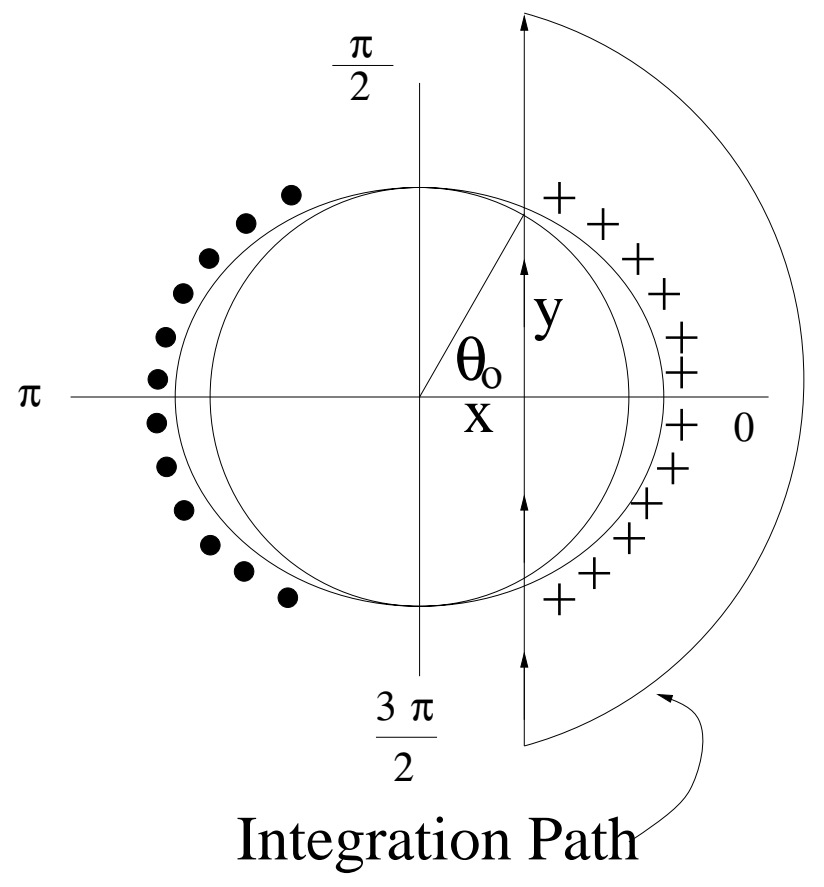

Figure 4.15: Integration path inside the $\cos (\theta)$ dipole magnet.

field is only dependent on the current delivered through the deflection magnet and the radius of the current windings. Where we have assumed that the contribution to the line integral is zero outside the current winding.

$$
\int \mathrm{B} \cdot \mathrm{dl}=\frac{8 \pi}{\mathrm{c}} \frac{\mathrm{I}}{\mathrm{R}}
$$


Chapter 5

\section{Experimental Results}




\section{$5.1 \quad$ Introduction}

In this section we present the results of our experimental beam dynamics studies of the Next Generation Photoinjector (NGP) conducted at the Brookhaven Accelerator Test Facility (ATF). A description of the ATF accelerator beam-line, laser, and computer control system will be presented. This is followed by the results of our beam dynamics studies which include quantum efficiency studies for both copper $(\mathrm{Cu})$ and magnesium (Mg) photoemitting materials. Electron beam energy as a function of accelerating field gradients and laser injection phase will be discussed along with dark current studies. In the low charge regime, experimental results of transverse normalized rms emittance as a function of solenoidal magnetic field, charge, and injection phase will then be presented. Longitudinal phase space experimental results will also be analyzed, including the electron beam's energy spread and bunch length. Finally, cathode magnetic field and multi-pole field asymmetries studies will be presented.

These experimental results will be used to make a quantitative prediction of the thermal emittance of the electron beam. Emittance measurements will be presented along with electron bunch length measurements in the $1 \mathrm{nC}$ high charge regime.

\section{$5.2 \quad$ Facility Overview}

The Next Generation Photoinjector, depicted in Fig. 5.1, has been commissioned at the Brookhaven Accelerator Test Facility as the electron source for beam dynamics studies, laser acceleration, and free electron laser experiments [47]. The NGP consists of the symmetrized BNL/SLAC/UCLA 1.6 cell S-band photocathode rf gun powered by an XK-5 klystron, and it is equipped with a single emittance compensation solenoidal magnet. There is a $78.55 \mathrm{~cm}$ drift space between the exit of the $\mathrm{rf}$ gun and the input coupler of the first of two SLAC three-meter traveling wave accelerating sections. Located along the low energy drift space is a copper mirror that can be used in both transition radiation studies and laser alignment. A beam profile monitor/Faraday plate is located $66.4 \mathrm{~cm}$ from the cathode plane. The Faraday plate is used for charge measurements and, when combined with charge measurements 


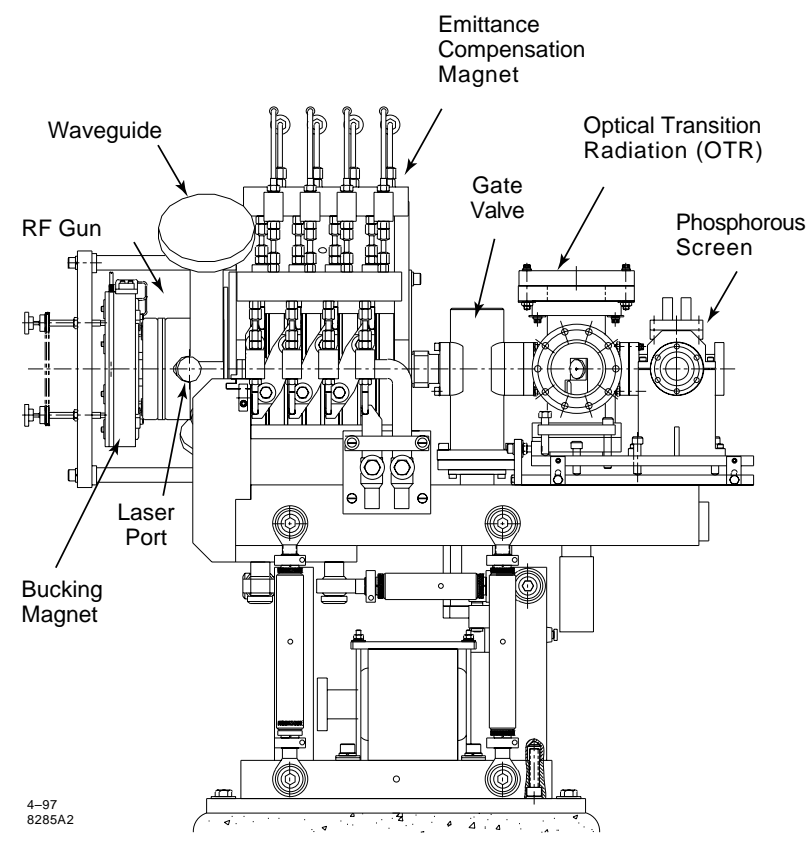

Figure 5.1: The Next Generation Photoinjector beam line layout.

conducted at the end of the high energy beam transport system, it allows for the experimental verification of $100 \%$ beam transmission. The beam profile monitor used in combination with the $\cos (\theta)$ deflection magnet located in the bore of the emittance compensation magnet can measure the energy of the electron beam out of the 1.6 cell rf gun.

\subsubsection{Linac System}

The ATF booster, Fig 5.2, consists of two SLAC three-meter traveling wave linacs powered by a second XK-5 klystron. The high energy beam transport system consists of nine quadrupole magnets, an energy spectrometer along with an energy selection slit, and a high-energy Faraday cup. Diagnostics located in the high energy transport section consist of beam profile monitors and strip lines. The strip lines can be used as an on-line laser/rf phase stability monitor. A block diagram of the laser, linac, and rf system is shown in Fig. 5.3.

A beam profile screen, HPOP1, is located at the output of the second linac section. 


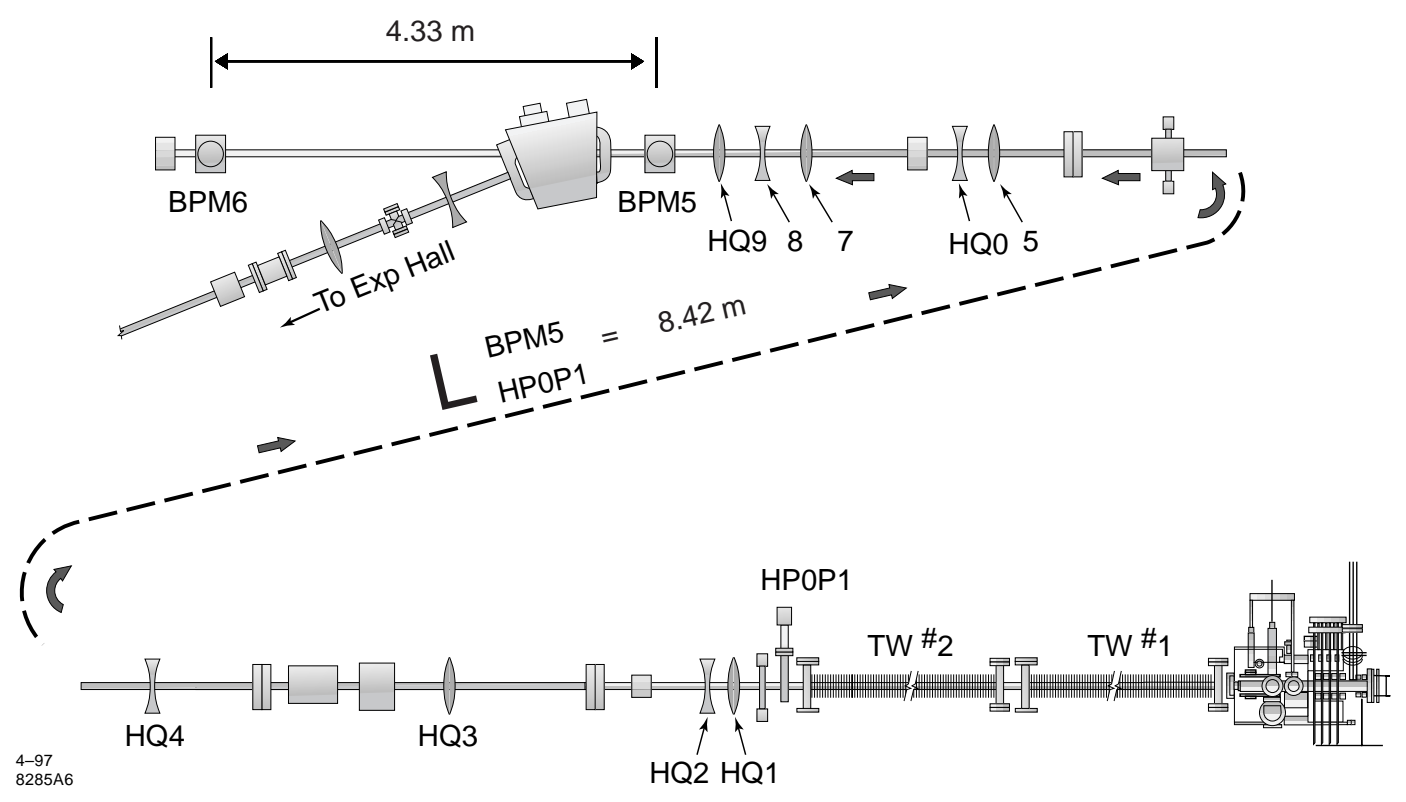

Figure 5.2: ATF beam-line

The beam is then transported, $8.42 \mathrm{~m}$, to the first of two beam profile screens, BPM5 and BPM6 used to conduct our two screen emittance measurements. The distance between BPM5 and BPM6 is $4.33 \mathrm{~m}$.

\subsubsection{Laser System}

The drive laser is an Nd:YAG master oscillator/power amplifier system with the diode pumped oscillator mode locked at 81.6 MHz, which produces a 21 psec Full Width Half Maximum (FWHM) pulse with an average power of $100 \mathrm{~mW}$ at a rate of $3 \mathrm{~Hz}$. Gated pulses seed two flash-lamp pumped multi-pass amplifiers and are subsequently frequency quadrupled. This nonlinear process leads to a reduction in the laser pulse length by a factor of two. Fig. 5.4 represents the layout of the front end of the laser system.

The $266 \mathrm{~nm}$ laser pulse is transported to the $\mathrm{rf}$ gun area via a 20 meter long evacuated pipe. The ultraviolet uv laser beam transport system in the rf gun hutch includes an aperture, a set of telescoping lenses, and a limiting aperture. The limiting 


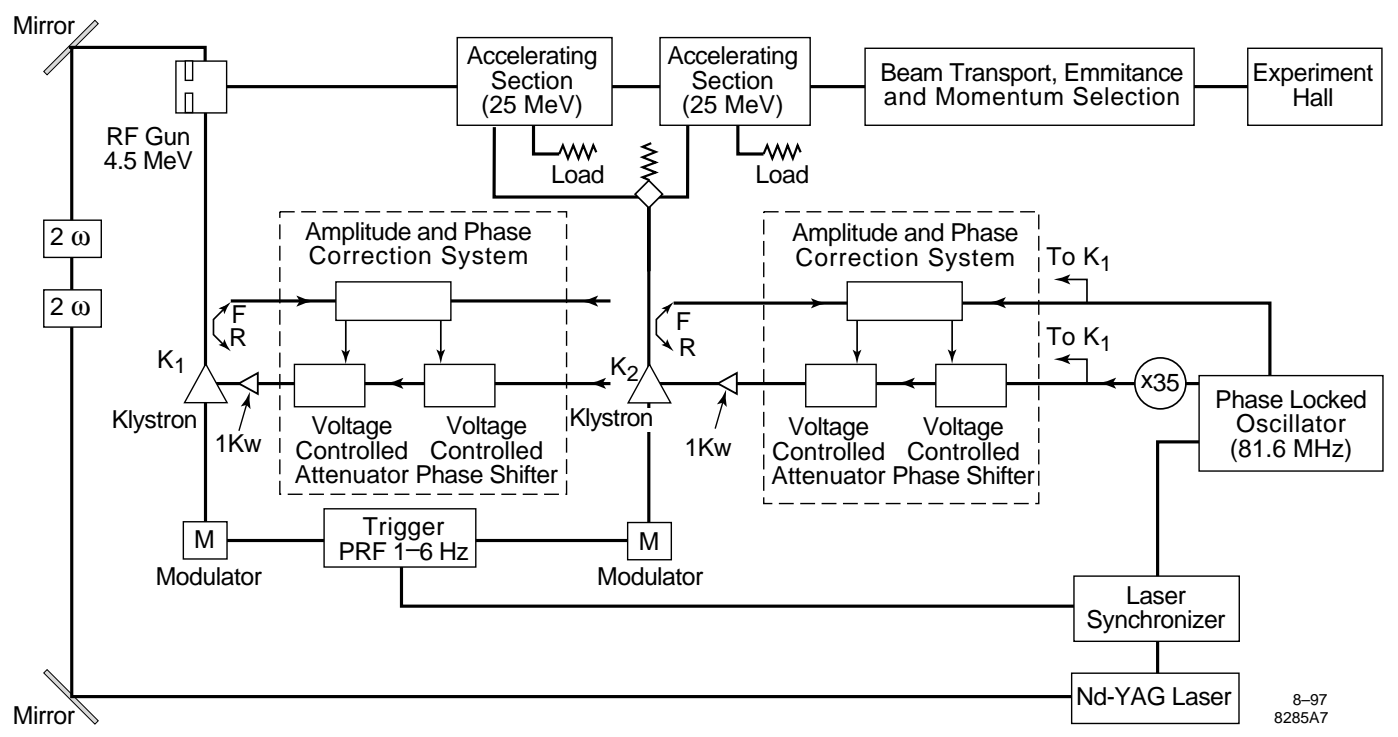

Figure 5.3: Linac,laser, and rf system block diagram.

aperture is imaged onto the cathode with a spherical lens and a pair of Littrow prisms which compensate for the anamorphic magnification introduced by the $72^{\circ}$ incidence angle on the cathode. The time slew across the cathode caused by the oblique laser incidence is corrected by using a diffraction grating. Relay imaging is used throughout the optical transport system to improve the beams pointing stability. The laser beam overfills the limiting aperture, consequently the transverse profile of the beam is a truncated Gaussian. When the truncated Gaussian laser pulse is convolved with the cathode's 2-D quantum efficiency, QE(x,y), the electron beam's transverse space charge distribution is produced. The laser spot size on the cathode is $2 \mathrm{~mm}$ in diameter. The layout of the uv gun hutch is shown in Fig. 5.5.

\subsubsection{Control System}

VISTA [48] control system software, which has a graphical user interface, is used at the ATF for remote operation of the rf, laser, magnet power supplies, and all profile screens, both pneumatic pop-ins and stepping motor varieties. The video signal switchboard can be manually or computer controlled, allowing for beam profile data to be viewed at any remote location. For this dissertation, all data that involves 


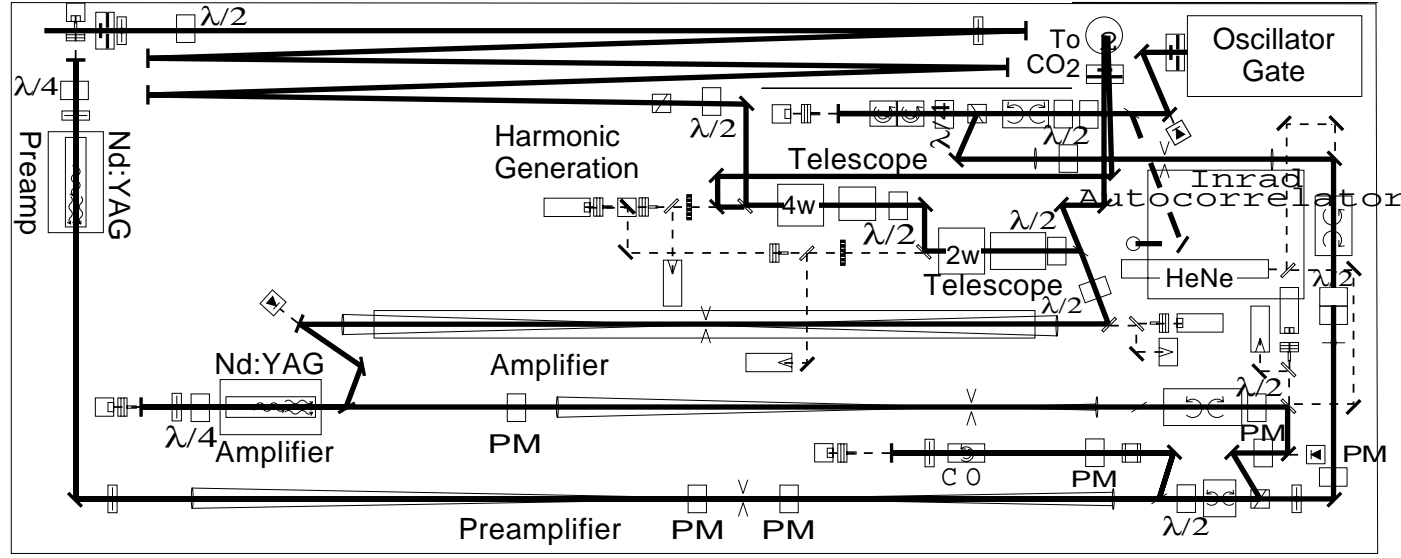

Laser head

ㄱ. Beamsplitter (on

kinematic base)

$\square \square$ Mirror (on permanent base)

(⿴) Vertical cube polarizer

$\square$ Horizontal cube polarizer

$\square \square$ Horizontal thin film polarizer

4 Alignment Camera

Windowless Camera

-Z Interlock shutter

(3) Pockel's cell

$\square$ Photodiode
PM Power meter holder

$\lambda / 4$ Quarter wave plate

$\lambda / 2$ Half wave plate

Faraday rotator

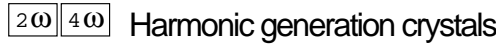

Primary relay aperture

A Alignment iris

$\square$ Color separating filter

$\gg$ Spatial filter

$\checkmark \mathrm{J} 3 / 4$ joulemeter head

$\varangle$ J25 joulemeter head

0 Periscope

$4-97$
$8285 \mathrm{~A} 5$

Figure 5.4: Laser system front end. 


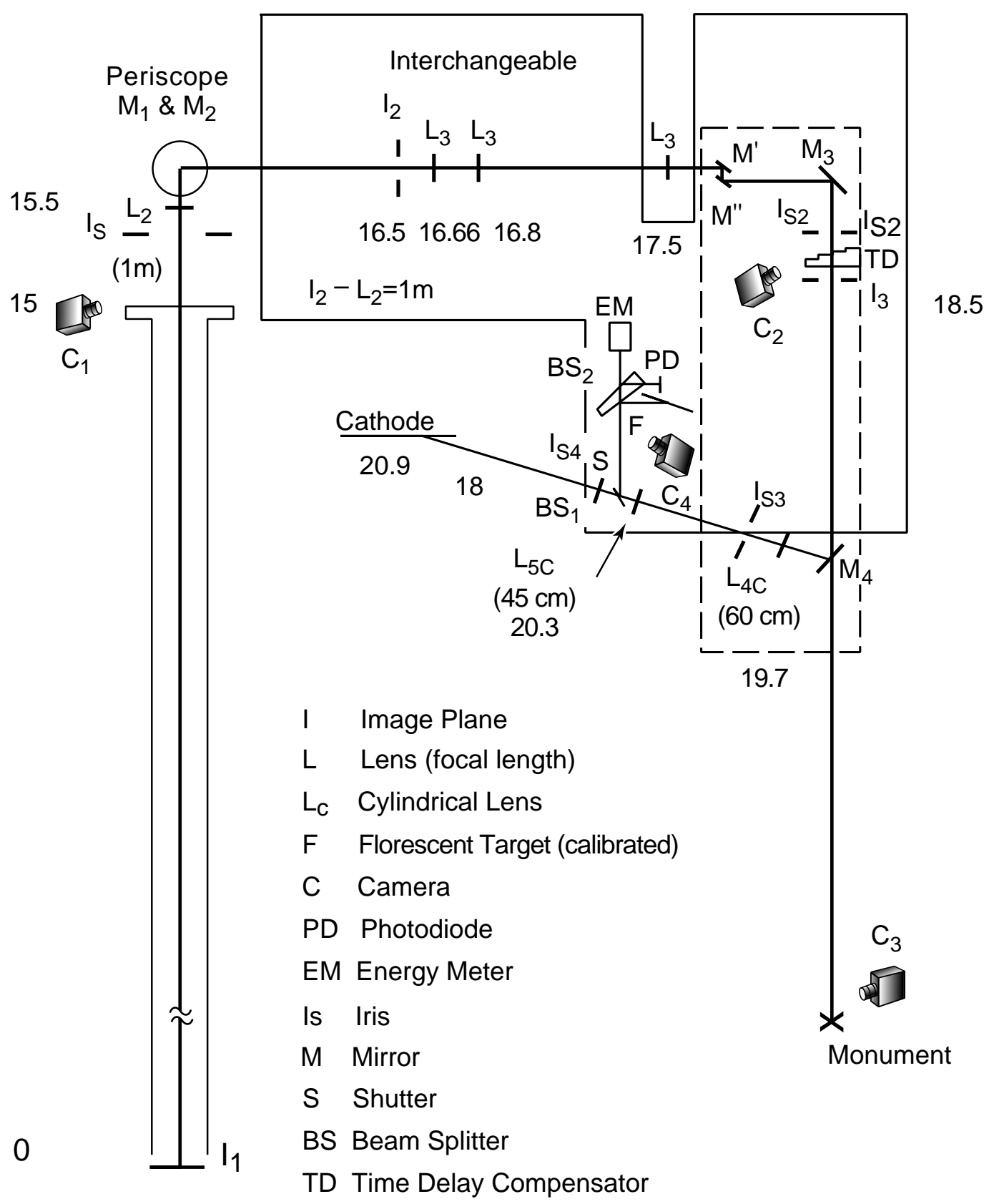

Notes: 1 . The distance from $\mathrm{I}_{3}$ to the cathode, target $\mathrm{F}$, and the monument is 2.4 meters.

2. The dashed figure with thick dashes represents the concrete pad. The other figure with thin dashes is a $6^{\prime} \times 2^{\prime}$ bread board.

Figure 5.5: uv gun hutch layout. 
a video image was routed to a LBA-100A Spiricon laser beam analyzer. The LBA$100 \mathrm{~A}$ was used to measure and store the electron beam image on disk for off-line analysis.

\subsection{Two Screen Emittance Measurement}

\subsubsection{Theory of Emittance Measurement}

Element beam transport theory [49] can be used to related the $\sigma$ matrix to the Twiss matrix. This relationship is shown in Eq. 5.1.

$$
\sigma=\left[\begin{array}{ll}
\sigma_{11} & \sigma_{21} \\
\sigma_{12} & \sigma_{22}
\end{array}\right]=\epsilon\left[\begin{array}{cc}
\beta_{\mathrm{T}} & -\alpha_{\mathrm{T}} \\
-\alpha_{\mathrm{T}} & \gamma_{\mathrm{T}}
\end{array}\right]
$$

where $\epsilon$ is the beam emittance, and $\alpha_{\mathrm{T}}, \beta_{\mathrm{T}}$, and $\gamma_{\mathrm{T}}$ are the Twiss parameters. The Twiss parameters are related to each other by the relation in Eq. 5.2.

$$
\beta_{\mathrm{T}} \gamma_{\mathrm{T}}-\alpha_{\mathrm{T}}^{2}=1
$$

Assuming that a beam waist is produced, $\alpha_{\mathrm{T}}=0$, the 2-D volume of the transverse phase space is defined in Eq. 5.3.

$$
\mathrm{V}_{2}^{\rho}=\pi \sqrt{\operatorname{det} \sigma}=\pi \sqrt{\sigma_{11} \sigma_{22}}=\pi \epsilon
$$

It should be noted that there is possibly some confusion over the symbol $\pi$ in the unit of the transverse normalized rms emittance. It is customary to include the $\pi$ to indicate that the phase space are reported is the product of the semi-major axis and semi-minor axis of the phase space and not the area of the phase space, which for and upright ellipse is $\pi \mathrm{ab}$.

Eq. 5.4 can be used to calculate the $\mathrm{i}^{\text {th }} \mathrm{rms}$ beam size at a beam profile screen. This equation can be inverted and with minimum of three spot sizes for different beam line tunes we can find the least squares fit to $\sigma_{11}, \sigma_{22}$, and $\sigma_{12}$. If we assume that the beam is at a waist, where a beam profile screen is located, then $\sigma_{12}=0$. This 
allows the use of only two screens to measure the emittance, with some systematic error, which will be discussed below.

$$
\sigma_{\mathrm{i}, 11}=\mathrm{C}_{\mathrm{i}}^{2} \sigma_{11}+2 \mathrm{~S}_{\mathrm{i}} \mathrm{C}_{\mathrm{i}} \sigma_{12}+\mathrm{S}_{\mathrm{i}}^{2} \sigma_{22}
$$

where $\sigma_{i, 11}$ is the $i^{\text {th }}$ measured rms beam spot size squared. $\mathrm{S}_{\mathrm{i}}$ and $\mathrm{C}_{\mathrm{i}}$ are the sine and cosine like beam transport function.

Assuming that $\sigma_{12}=0$ (ie: an upright transverse phase space ellipse), then the maximum rms spot size is given by Eq. 5.5 and the maximum rms angular divergence is given by Eq. 5.6.

$$
\begin{aligned}
& \sigma_{\mathrm{g}, \mathrm{rms}}^{\mathrm{x}}=\sqrt{\beta_{\mathrm{T}} \epsilon_{\mathrm{g}, \mathrm{rms}}} \\
& \sigma_{\mathrm{g}, \mathrm{rms}}^{\mathrm{x}^{\prime}}=\sqrt{\gamma_{\mathrm{T}} \epsilon_{\mathrm{g}, \mathrm{rms}}}
\end{aligned}
$$

From Eq. 5.3 we find that the beams transverse normalized rms emittance is defined in Eq. 5.7. Noted that we have taken advantage of the fact that we have produced a beam waist, $\beta_{\mathrm{T}}=\frac{1}{\gamma_{\mathrm{T}}}$ and $\alpha_{\mathrm{T}}=0$.

$$
\epsilon_{\mathrm{n}, \mathrm{rms}}=\beta \gamma \sigma_{\mathrm{g}, \mathrm{rms}}^{\mathrm{x}} \sigma_{\mathrm{g}, \mathrm{rms}}^{\mathrm{x}^{\prime}}
$$

where $\beta$ and $\gamma$ are the relativistic correction factors and not the Twiss parameters.

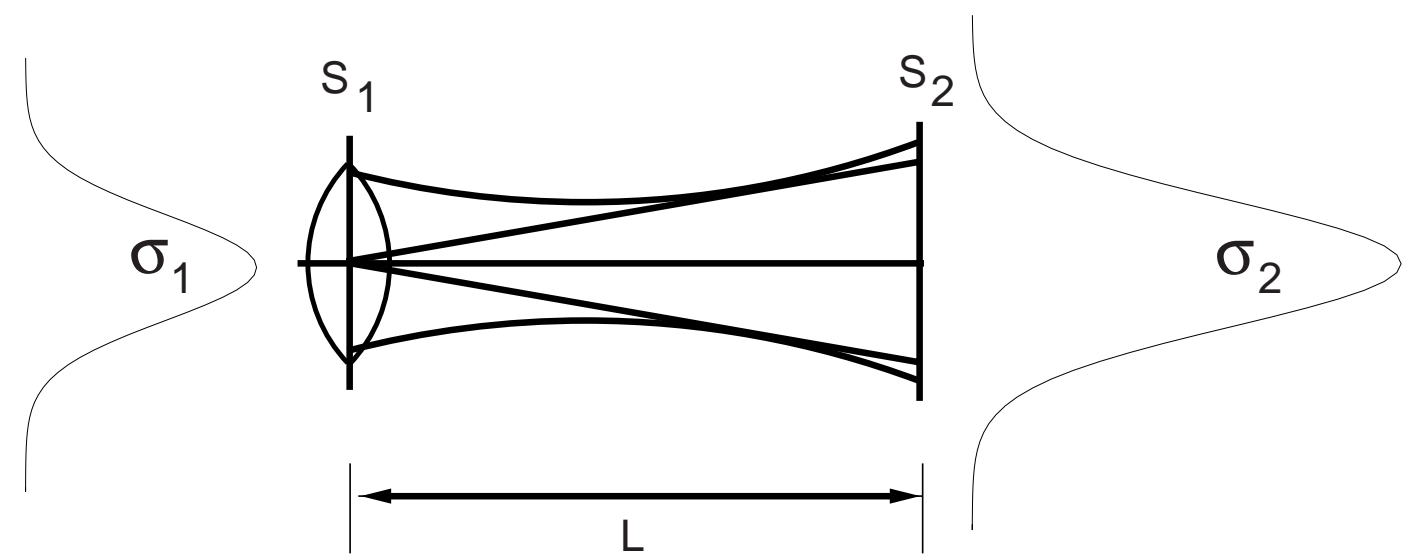

Figure 5.6: Graphical illustration of the two screen emittance measurement method. 
Emittance data was taken using the two screen method, which is graphically demonstrated in Fig. 5.6. Assuming that a profile screen, $\mathrm{S}_{1}$, is located in the middle of a quadrupole and another profile screen, $\mathrm{S}_{2}$, is located downstream a known distance $\mathrm{L}_{\mathrm{o}}$, the maximum angular divergence can be calculated using the rms spot size, $\sigma_{2}$, at the second screen and the drift distance $\mathrm{L}_{\mathrm{o}}$. Using the rms spot size, $\sigma_{1}$, at the first screen, along with the maximum angular divergence at that point, Eq. 5.8 gives an upper limit of the normalized rms emittance of the electron beam.

$$
\epsilon_{\mathrm{n}, \mathrm{rms}}=\beta \gamma \sigma_{1} \frac{\sigma_{2}}{\mathrm{~L}_{\mathrm{o}}}
$$

If the quadrupole lens is used to minimize the beam spot size at $\mathrm{S}_{2}$ then Eq. 5.8 is the measured normalized rms emittance and not an upper limit of the normalized rms emittance of the electron beam.

\subsubsection{Experimental Measurement Technique}

Each screen used to take spot size data was equipped with a remote controlled focusing/iris system combined with an 8-bit charged coupled detector (CCD) camera. The remote controlled iris prevents the beam image from saturating the CCD. The screens themselves have lines scribed into the phosphor with a $2.5 \mathrm{~mm}$ separation. These lines were used as calibration fiducials so that the beam size is known to an absolute reference for any camera zoom setting.

Multiple scattering effects were noted in some spot size images which adversely effected the results of our calculations. This effect was eliminated by cropping out the desired portion of the image which was subsequently analyzed in the manner described below. Background subtraction of the dark current was accomplished using the LBA-100A Spiricon laser beam analyzer.

Comparison of rms least squares fit, FWTM, and FWHM to the beam spot size data indicated that the FWHM was not significantly effected by the third (skew) and fourth (kurtosis) moments of the beam distribution. Therefore the electron beam images were analyzed off-line using a FWHM fit. This fitting routine integrates the pixels of the beam image in one dimension and removes any DC baseline. The FWHM 
points were found by linear interpolation around the FWHM pixel points. Using this method we were able to increase the resolution of our fitting routine. The beam profile was assumed to have a standard normal distribution and the normalized FWHM data was scaled using Eq. 5.9 to find $\sigma_{\mathrm{x}, \mathrm{y}}$.

$$
\mathrm{FWHM}=\sigma_{\mathrm{x}, \mathrm{y}} \sqrt{8 \ln (2)}
$$

The variance of the rms normalized emittance is calculated using Eq. 5.10, where $\epsilon_{\mathrm{n}, \mathrm{rms}}$ is the beam emittance defined in Eq. 5.8, and $\sigma_{\mathrm{x}_{1}}$ and $\sigma_{\mathrm{x}_{2}}$ are the transverse rms beam sizes at the first and second profile screen, respectively.

$$
\sigma_{\mathrm{u}}^{2}=\left(\frac{\partial \epsilon_{\mathrm{n}, \mathrm{rms}}}{\partial \sigma_{\mathrm{x}_{1}}}\right)^{2} \sigma_{\mathrm{x}_{1}}^{2}+\left(\frac{\partial \epsilon_{\mathrm{n}, \mathrm{rms}}}{\partial \sigma_{\mathrm{x}_{2}}}\right)^{2} \sigma_{\mathrm{x}_{2}}^{2}+2 \operatorname{cov}\left(\sigma_{\mathrm{x}_{2}}, \sigma_{\mathrm{x}_{2}}\right) \frac{\partial \epsilon_{\mathrm{n}, \mathrm{rms}}}{\partial \sigma_{\mathrm{x}_{1}}} \frac{\partial \epsilon_{\mathrm{n}, \mathrm{rms}}}{\partial \sigma_{\mathrm{x}_{2}}}
$$

Since shot to shot variations cannot be recorded at each profile screen for each individual electron beam shot, it is not possible to calculate the covariance in Eq. 5.10. Therefore, in the emittance results presented, we assume that the correlation term is zero.

\section{Experimental Procedure}

A beam waist is produced at a beam profile screen (BPM5) and allowed to drift to the second beam profile screen (BPM6) over a distance of $4.33 \mathrm{~m}$ using HQ9 as the final magnetic element, see Fig. 5.2. Spot size data, 25 shots, is recored at BPM5 them the screen is retracted and spot size data is recorded at BPM6 (25 shots). Beam parameters are monitored continually during the data run to insure beam stability. These include laser energy, electron bunch charge, rf amplitude in both the gun and linacs, and rf phase stability using an rf strip-line located in the high energy transport line. Finally after all spot size data is taken at BPM6, BPM5 is reinserted into the beam line to insure that the beam spot size has not changed not changed. If any instability is noted, all the data taken in that run is rejected, the problem corrected and the data run is started over again. 


\begin{tabular}{|l|c|}
\hline Error Source & Estimated $\epsilon_{\mathrm{n}, \mathrm{rms}}$ Error \\
\hline Phosphor Grain Size & $+5 \%$ \\
\hline Beam Waist versus Minimum Spot Size & $+32 \%$ \\
\hline Fitting Routine & $+16 \%$ \\
\hline
\end{tabular}

Table 5.1: Estimated value of the systematic errors

\subsubsection{Systematic Errors}

Table 5.1 list our estimate of the systematic errors in our transverse normalized rms emittance measurements. All the estimated systematic errors listed in Table 5.1 indicate that the measured emittance is an over estimate of the beams actual transverse normalized rms emittance.

The beam profile screen used for BPM5 and BPM6 have a resolution of $50 \mu \mathrm{m}$. Using the two screen emittance measurement method, we estimate that we have a systematic error of $+5 \%$.

Using PARMELA [3] to simulate the two screen emittance measurement method, we see $\mathrm{a}+32 \%$ systematic error in the measured emittance versus the simulated beams emittance.

The fitting routine, FWHM to $\sigma_{x, y}$, induces an estimated systematic error of $+16 \%$. It should be pointed out that our fitting routine was selected since it is a robust fitting parameter, which is not significantly effected by the third (skew) and fourth (kurtosis) moments of the beam distribution.

Assuming that all the systematic error are uncorrelated we can estimate an upper limit of the systematic errors at $+36 \%$. This indicates that work must be directed in the are of advanced beam diagnostics.

\subsection{Quantum Efficiency Studies}

The quantum efficiency of a photocathode is defined by the ratio of the number of electrons, $\# \mathrm{~N}_{\mathrm{e}}$, produced to the number of incident photons $\# \mathrm{~N}_{\gamma}$. In Eq. 5.11 this ratio is expressed as a ratio of the beam charge and laser energy 


$$
\mathrm{QE}=\frac{\# \mathrm{~N}_{\mathrm{e}}}{\# \mathrm{~N}_{\gamma}}=\left[\frac{\mathrm{h} \mathrm{c}}{\mathrm{e} \lambda}\right]\left[\frac{\mathrm{Q}_{\mathrm{t}}}{\mathrm{E}_{\gamma}}\right]=4.645 \times 10^{-3}\left[\frac{\mu \mathrm{J}}{\mathrm{nC}}\right]\left[\frac{\mathrm{Q}_{\mathrm{t}}}{\mathrm{E}_{\gamma}}\right]
$$

where $\mathrm{h}$ is Planks constant, $\mathrm{c}$ is the speed of light, e is the electron charge, $\lambda$ is the laser wavelength, $\mathrm{Q}_{\mathrm{t}}$ is the total electron bunch charge in $\mathrm{nC}$, and $\mathrm{E}_{\gamma}$ is the laser pulse energy in $\mu \mathrm{J}$.

During the commissioning of the BNL/SLAC/UCLA 1.6 cell S-band emittance compensated photoinjector, QE studies were conducted on two different types of cathode materials, OFHC grade II $\mathrm{Cu}$ and $99.8 \% \mathrm{Mg}$.

\subsubsection{Cathode Preparation Technique}

Preparation of the cathode plate was in accordance with standard ultra-highvacuum (uhv) practices. The center portion of the cathode plate was initially polished with a 600 grit soft polishing cloth. $9 \mu \mathrm{m}$ Buehler diamond polish was then applied followed by the use of $6 \mu \mathrm{m}$ diamond polish, and, finally, $1 \mu \mathrm{m}$ grit was used to polish the photocathode. An ultrasonic bath of hexane was utilized after the final $1 \mu \mathrm{m}$ polishing step. The cathode plate was then placed in an evacuated glass bell jar for transport to the ATF for a high temperature bake-out and installation [50].

During the course of our experimental beam studies at the ATF, the need for higher total charge became apparent. Two different in situ laser cleaning techniques were used. The application of rf power into the gun is the major difference between the two techniques. The initial choice of vacuum laser cleaning over rf assisted laser cleaning was dictated by our overriding concern not to damage the photocathode. Since vacuum laser cleaning cannot initiate a super charge event, it is considered the least damaging of the two laser cleaning techniques. Therefore, it was used first.

\section{Vacuum Laser Cleaning}

For vacuum laser cleaning, the diameter of the laser spot size is decreased from $2 \mathrm{~mm}$ to $500 \mu \mathrm{m}$. The initial total energy in one laser pulse is $1 \mu \mathrm{J}$. The laser energy was increased in $1 \mu \mathrm{J}$ steps until a vacuum fluctuation in the rf gun was seen or the laser energy reached $15 \mu \mathrm{J}$. The laser spot is then scanned over a $\pm 1 \mathrm{~mm}$ square that 
is centered on the geometrical center of the gun, with step sizes of 125 and $83 \mu \mathrm{m}$ in the horizontal and vertical directions, respectively. This ensures that each point in the active photoemitting area will receive multiple shots of laser energy. During the vacuum laser cleaning, the gate valve seen in Fig. 5.1 is closed and the rf gun vacuum pressure is continually monitored for vacuum bursts. Fig. 5.7 shows the improvement in QE before and after vacuum laser cleaning for the magnesium plug cathode plate. Plot $\mathrm{A}$ in Fig. 5.7 is the original QE of the $\mathrm{Mg}$ cathode plug without laser cleaning and plot $\mathrm{B}$ is the $\mathrm{QE}$ after vacuum laser cleaning.

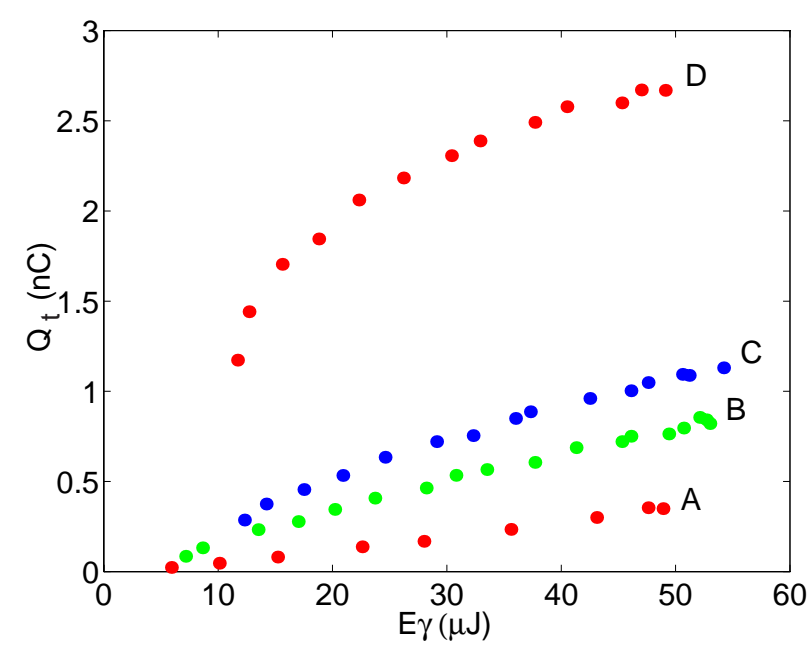

Figure 5.7: Total charge versus laser energy before and after laser cleaning. The different data sets are described in the text.

\section{rf Laser Cleaning}

Laser Assisted Explosive Electron Emission (LAEEE) [51] is a variant of the vacuum laser cleaning in that a field gradient of $70 \frac{\mathrm{MV}}{\mathrm{m}}$ is applied to the gun while conducting the scanning method discussed in the previous section, with a $500 \mu \mathrm{m}$ diameter laser spot. The vacuum gate valve in Fig 5.1 is now open.

Initially, each horizontal and vertical position is irradiated with $15 \mu \mathrm{J}$ of laser energy for five consecutive shots at a $3 \mathrm{~Hz} \mathrm{PRF}$. If three consecutive super charge events are not produced, the uv laser shutter is closed and the laser energy is increased by $2.5 \mu \mathrm{J}$. This process is repeated at the same cathode position until we achieve 
three consecutive super charge events. A super charge event occurs when a plasma is produced on/near the photocathode surface and 100's of nC of charge can be produced from the gun over a rf macro pulse. When the three consecutive super charge events have occurred, the uv laser shutter is closed and the laser spot is scanned to a new position. The step sizes were 125 and $83 \mu \mathrm{m}$ in the horizontal and vertical directions, respectively. The laser energy is decreased to $15 \mu \mathrm{J}$, the uv shutter is opened, and the process is repeated until the $\pm 1.25 \mathrm{~mm}$ square area of the photocathode is cleaned.

Plot $\mathrm{C}$ in Fig. 5.7 represents the charge produced from the $\mathrm{Mg}$ cathode after LAEEE. After repeated rf laser cleaning, we were able to produce up to $2.5 \mathrm{nC}$ in a single electron bunch, represented by plot D in Fig. 5.7. In Section 5.4.2 we will present data that show that the 2-D variation of the cathode $\mathrm{QE}, \mathrm{QE}(\mathrm{x}, \mathrm{y})$, is reduced with a systematic application of LAEEE.

\subsubsection{Cathode Studies}

An OFHC grade II copper cathode was used for all emittance compensation beam dynamics studies. In the following subsections, the results of our polarization, quantum efficiency (QE), and QE(x,y) studies, are presented for both the OFHC II grade $\mathrm{Cu}$ cathode and a $\mathrm{Mg}$ cathode. All of these results were obtained in the low energy diagnostics section of the NGP depicted in Fig. 5.1 with the laser injection phase, $\Phi$, set to the crest of the rf accelerating field.

A Schottky phase scan, which is a plot of $Q_{t}$ versus $\Phi_{l 1 r f}$ for a constant laser energy, is conducted to find the $\mathrm{rf}$ phase which corresponds to the maximum charge. This phase is defined as the rf crest. In Fig. 5.8, the rf crest occurs at $245^{\circ}$ with respect to the low level rf system. There is not a one to one correspondence between the low level rf system phase and the photoelectron production on/off phases, which is $0^{\circ}$ and $180^{\circ}$ respectively. To get a meaningful measure of the absolute phase, one must know where the crest of the rf occurs and the scaling of the low level rf system with respect to photoelectron production on/off phases. The sharp drop in the charge versus phase, between $245^{\circ}$ and $290^{\circ}$, of the Schottky scan in Fig. 5.8 is due to beam transport issues when the laser injection phase is behind the crest of the rf. 


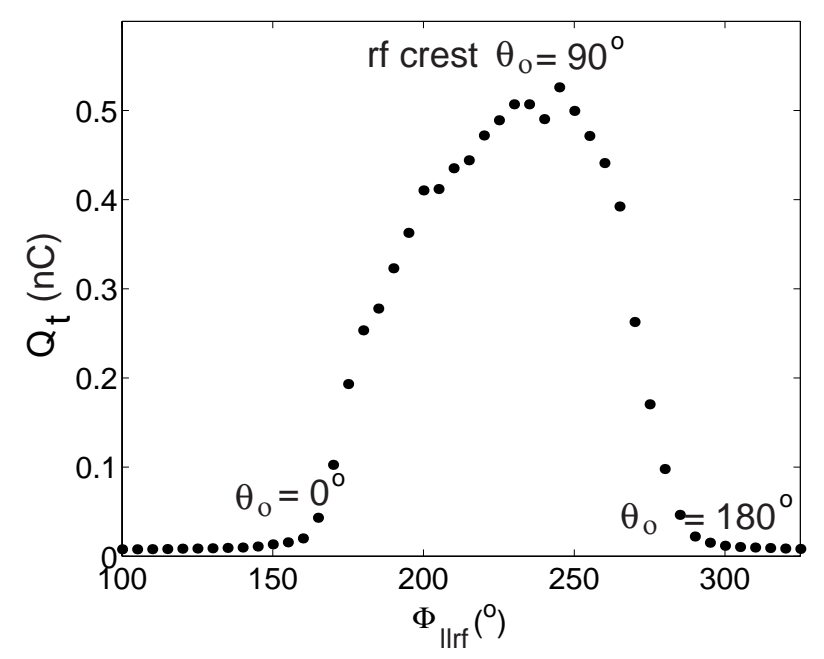

Figure 5.8: Schottky phase scan.

\section{QE Versus Laser Polarization, $\Omega_{\gamma}$}

The effect of total electron bunch charge as a function of laser polarization, $\Omega_{\gamma}$, was investigated experimentally, since the laser strikes the cathode at a grazing incidence angle of $72^{\circ}$ with respect to cathode plate normal vector. This effect can be analyzed in the following manner. The laser light will penetrate the cathode surface up to a skin depth of the laser wavelength. By adjusting the laser light polarization to maximize the number of photons that penetrate the cathode material, we can excite more electrons from the conduction band into the vacuum.

Measurements of the laser lights polarization with respect to the cathode normal vector were not accomplished. Therefore the laser polarization, $\Omega_{\gamma}$, is a relative polarization of the laser light.

Installation of a new photocathode requires that the laser polarization be set to maximize the total electron bunch charge photoemitted by that cathode. Figs. 5.9 and 5.10 are plots of the total electron bunch charge, $Q_{t}$, versus laser polarization, $\Omega_{\gamma}$, for a given laser energy, $\mathrm{E}_{\gamma}$, with the laser injection phase set at the rf crest, $\theta_{0}=90^{\circ}$. Using a least squares fit, this data shows that the maximum electron bunch charge occurs with a laser polarization of $\Omega_{\gamma}=61.5^{\circ}$ and $\Omega_{\gamma}=65^{\circ}$ for $\mathrm{Cu}$ and $\mathrm{Mg}$, respectively. 


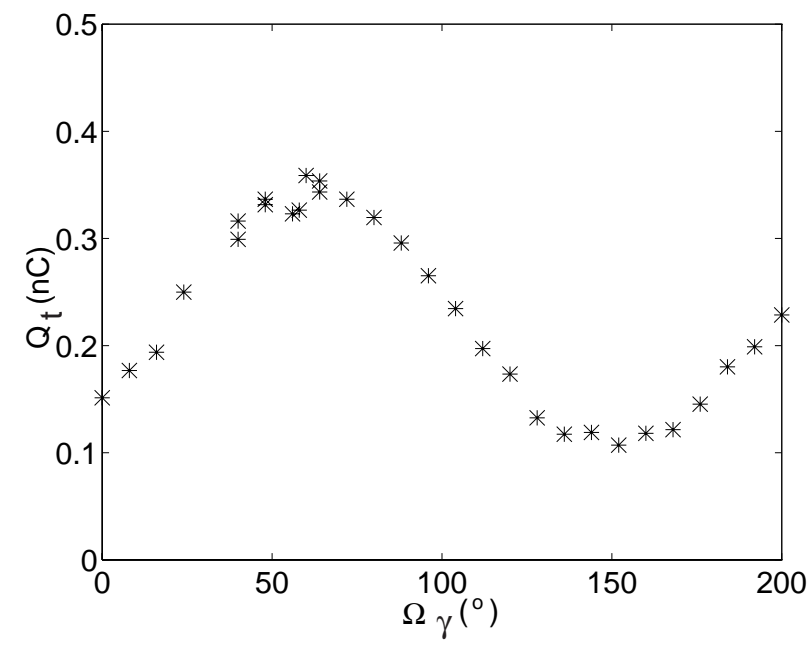

Figure 5.9: Total charge versus polarizer angle for the $\mathrm{Cu}$ cathode.

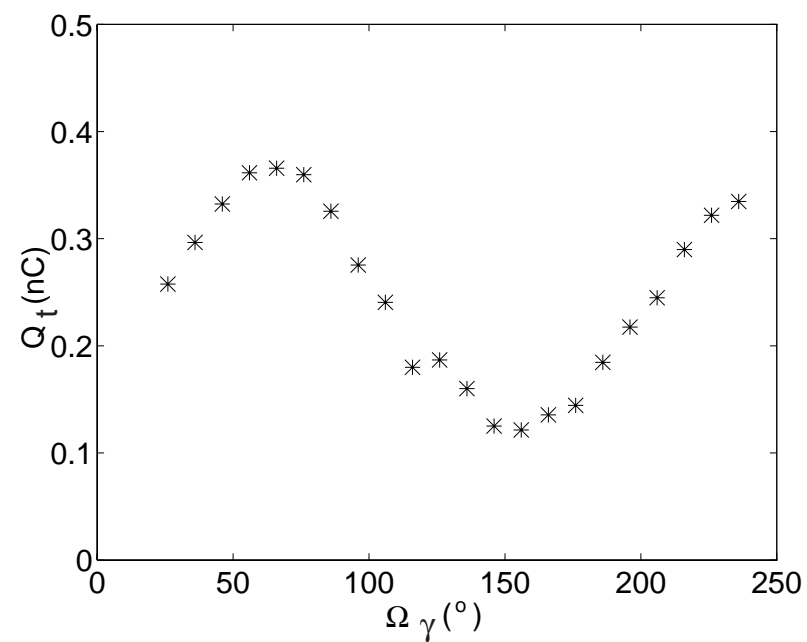

Figure 5.10: Total charge versus polarizer angle for Mg cathode. 


\section{$\mathrm{QE}$}

During commissioning of the 1.6 cell rf gun, QE measurements were accomplished on both the $\mathrm{Cu}$ and $\mathrm{Mg}$ cathodes. Fig. 5.11 represents the total charge versus laser energy for the OFHC grade II $\mathrm{Cu}$ cathode, with a laser injection phase set on the crest of the rf. Using Eq. 5.11 we find that the measured quantum efficiency is $\mathrm{QE}=4.5 \times 10^{-5}$. The maximum available charge for a laser injection phase of $45^{\circ}$ was limited to $\mathrm{Q}_{\mathrm{t}} \leq 0.333 \mathrm{nC}$. This $\mathrm{QE}$ result is an order of magnitude lower than previous published copper QE results [44].

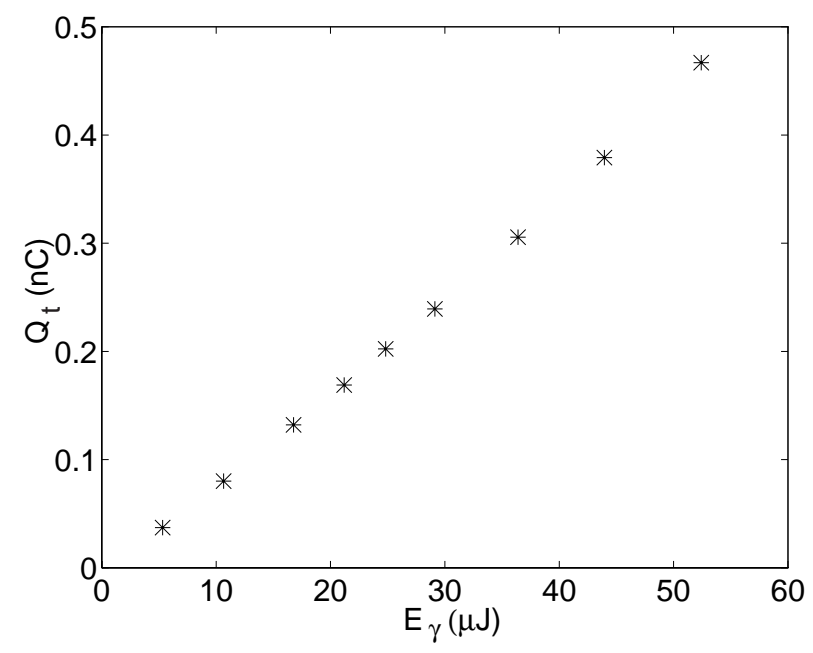

Figure 5.11: Total charge versus laser energy for the copper cathode used in the low charge emittance studies.

The Mg cathode, charge versus laser energy plot after systematic LAEEE, is shown in Fig. 5.12. Using a laser energy of $30.4 \mu \mathrm{J}$ and a total electron bunch charge of 2.3 $\mathrm{nC}$ in Eq. 5.11, the QE for the 99.8\% Mg cathode is $\mathrm{QE}=3.5 \times 10^{-4}$. The charge free accelerating field gradient was $100 \frac{\mathrm{MV}}{\mathrm{m}}$ at the cathode. This QE result is an order of magnitude larger than that of the OFHC grade II Cu cathode.

The initial QE of the $\mathrm{Mg}$ cathode plug was less than that of the $\mathrm{Cu}$ cathode. Only after LAEEE cleaning did the $\mathrm{Mg}$ cathode plug QE improve. The data in Fig. 5.12 indicate that at high charge, the accelerating field at the cathode reaches a space charge limit, since as the laser energy is increased the total electron bunch 
charge reaches saturation. In effect, the longitudinal space charge field of the emitted electrons at the head of the bunch suppresses the longitudinal accelerating field of the rf. The effective QE of the cathode at the end of the laser pulse is decreased due to the suppression of the Schottky enhancement.

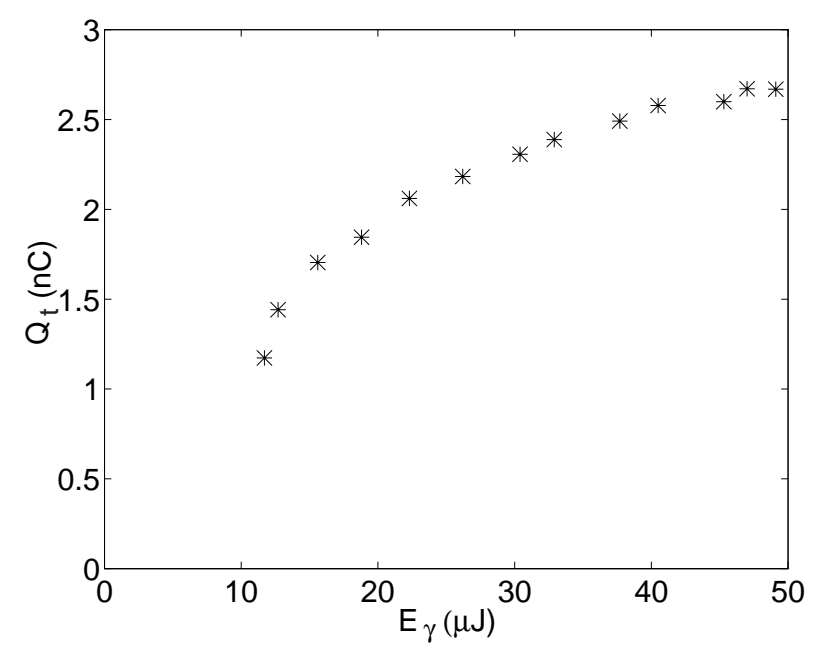

Figure 5.12: Total charge versus laser energy for the Mg cathode.

\section{QE Versus Cathode Position, QE(x,y)}

Simulations indicate that the emittance compensation process is optimized if the transverse electron beam charge distribution is uniform. Variations in the laser transverse profile and the 2-D quantum efficiency will be manifested in a degradation of the emittance compensation process. We have studied the variation in the cathode $\mathrm{QE}$ as a function of position for both $\mathrm{Cu}$ and $\mathrm{Mg}$ photocathodes used in the 1.6 cell rf gun.

Fig. 5.13 represents the 2-D variation in the $\mathrm{Cu}$ cathode's QE. This data indicates that there is a $25 \%$ variation in the quantum efficiency across the $\mathrm{Cu}$ cathode. Assuming that the transverse laser profile is a flat-top, this data represents the transverse electron bunch charge distribution, which degrades the emittance compensation process. Beam dynamics simulations, which included perturbations in the uniform transverse charge distribution show a $40 \%$ increase in $\epsilon_{\mathrm{n}, \mathrm{rms}}$ [19]. 


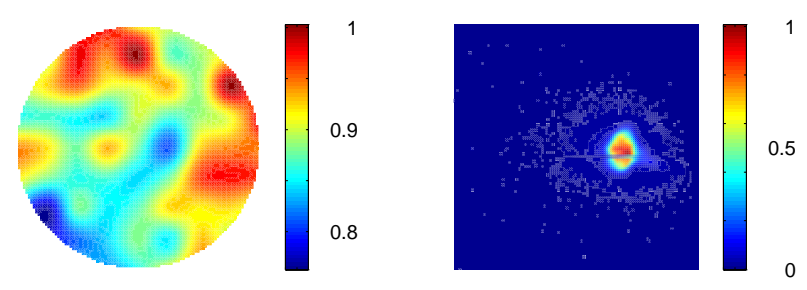

Figure 5.13: Normalized charge emission of a copper $(\mathrm{Cu})$ cathode of $1 \mathrm{~mm}$ radius with no laser cleaning.

Fig. 5.14 represents the 2-D variation in the Mg cathode's QE before and after LAEEE cleaning. Before LAEEE cleaning, there is an order of magnitude variation across the Mg cathode. After LAEEE cleaning, this variation is reduced by a factor of two, but is still worse than in the $\mathrm{Cu}$ cathode case.
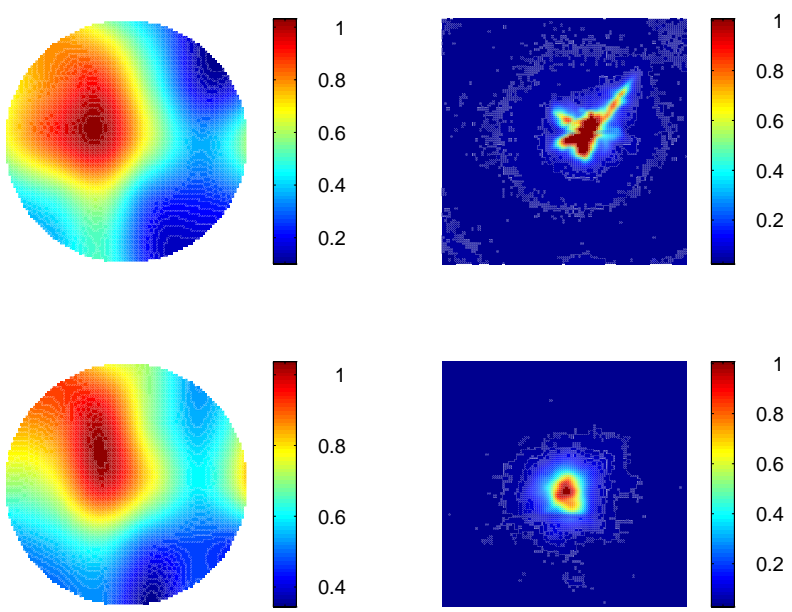

Figure 5.14: Normalized charge emission of a magnesium (Mg) cathode of $1 \mathrm{~mm}$ radius before and after LAEEE cleaning. 


\subsection{Low Energy Beam Studies}

\subsubsection{Electron Beam Energy, $\gamma \mathrm{m}_{0} \mathrm{c}^{2}$, Versus Accelerating Field Gradients, $\mathrm{E}_{0}$, and Laser Injection Phase, $\theta_{0}$}

PARMELA [3] simulations in Section 2.4.2 indicate that a field gradient of 143 $\frac{\mathrm{MV}}{\mathrm{m}}$ is optimal for the emittance compensation process. Therefore it is necessary to measure the electron beam energy to insure that the 1.6 cell gun can operate at these high field gradients. The electron beam energy, $\gamma \mathrm{m}_{\mathrm{o}} \mathrm{c}^{2}$, was measured in the low energy drift between the exit of the 1.6 cell rf gun and the profile screen located 66.5 $\mathrm{cm}$ from the cathode, see Fig. 5.1. These studies utilize the $\cos (\theta)$ deflection magnet, located in the bore of the emittance compensation magnet, as described in Section 4.5 of Chapter 4.

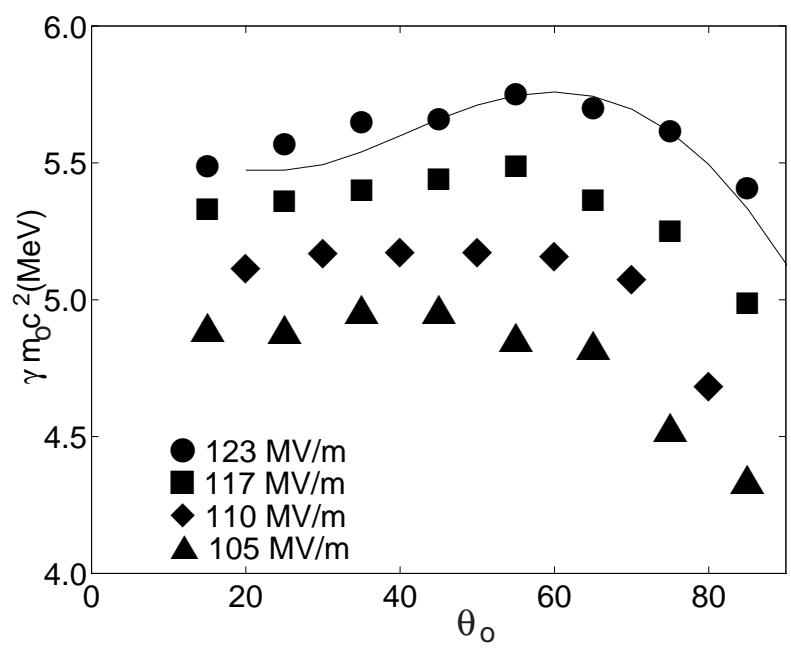

Figure 5.15: Electron beam energy versus laser injection phase for four different cathode fields.

The electron beam energy, $\gamma \mathrm{m}_{\mathrm{o}} \mathrm{c}^{2}$, was measured as a function of laser injection phase, $\theta_{o}$, at four different accelerating gradients, $\mathrm{E}_{\mathrm{o}}$. The data from these experiments are plotted in Fig. 5.15 where the crest of the $\mathrm{rf}$ is $90^{\circ}$.The solid line in Fig. 5.15 represents the reference particle energy from a series of PARMELA simulation. The field gradients list in Fig. 5.15 were calculated from PARMELA simulations. 


\subsubsection{Field Enhancement Factor $\beta$}

In Section 2.4.2 of Chapter 2 our PARMELA simulation of $\epsilon_{\mathrm{n}, \mathrm{rms}}$ versus $\mathrm{E}_{\mathrm{o}}$ and $\mathrm{B}_{\mathrm{z}}$ indicate that the emittance compensation process is optimized for an accelerating field gradient of $\mathrm{E}_{\mathrm{o}}=143 \frac{\mathrm{MV}}{\mathrm{m}}$. Excessive field emission current can limit the maximum field gradient that can be achieved in an rf cavity. A series of field emission current studies were conducted, as a function of accelerating field gradient, to find the maximum field level where reliable operation is ensured.

\section{Theory}

The field emission current for an rf cavity has been theoretically derived [52] and is given by Eq. 5.12

$$
\overline{\mathrm{I}}_{\mathrm{F}}=\frac{5.7 \times 10^{-12} \times 10^{4.52 \phi_{\mathrm{o}}^{-0.5}}}{\phi_{\mathrm{o}}^{1.75}} \mathrm{~A}_{\mathrm{eff}}\left(\beta \mathrm{E}_{\mathrm{o}}\right)^{2.5} \mathrm{e}^{-\frac{6.53 \times 10^{9} \times \phi_{0}^{1.5}}{\beta \mathrm{E}_{\mathrm{o}}}}
$$

where $\phi_{\mathrm{o}}$ is the work function of the structure in $\mathrm{eV}, \mathrm{E}_{\mathrm{o}}$ is the peak macroscopic field amplitude in $\mathrm{V} / \mathrm{m}, \beta$ is the field enhancement factor, and $\overline{\mathrm{I}}_{\mathrm{F}}$ is the average dark current in Amps, from the effective field emitting area, $\mathrm{A}_{\text {eff }}$.

Plotting $\ln \left(\frac{\overline{\mathrm{I}}_{\mathrm{F}}}{\mathrm{E}_{0}^{2.5}}\right)$ as a function of $\frac{1}{\mathrm{E}_{\mathrm{o}}}$, shows that the slope of this line is given in Eq. 5.13. This allows for the determination of the field enhancement factor, $\beta$.

$$
\frac{\mathrm{d}\left(\ln \left(\frac{\overline{\mathrm{I}}_{\mathrm{F}}}{\mathrm{E}_{\mathrm{o}}^{2.5}}\right)\right)}{\mathrm{d}\left(\frac{1}{\mathrm{E}_{\mathrm{o}}}\right)}=\left[-6.53 \times 10^{9} \phi_{\mathrm{o}}^{1.5}\right] \frac{1}{\beta}
$$

\section{Experimental Results}

The 1.6 cell rf gun was initially conditioned up to a maximum field gradient of $150 \frac{\mathrm{MV}}{\mathrm{m}}$ with a $0.7 \mu$ sec rf macro pulse. Further conditioning was not attempted due to operational requirements of the ATF.

The data used in the Fowler-Nordheim plot, Fig. 5.16, was taken with a $2.53 \mu \mathrm{s}$ rf pulse length at six different accelerating gradients. A least squares fit to this data indicates that $\beta=62$, which is comparable to most normal conducting cavities. 


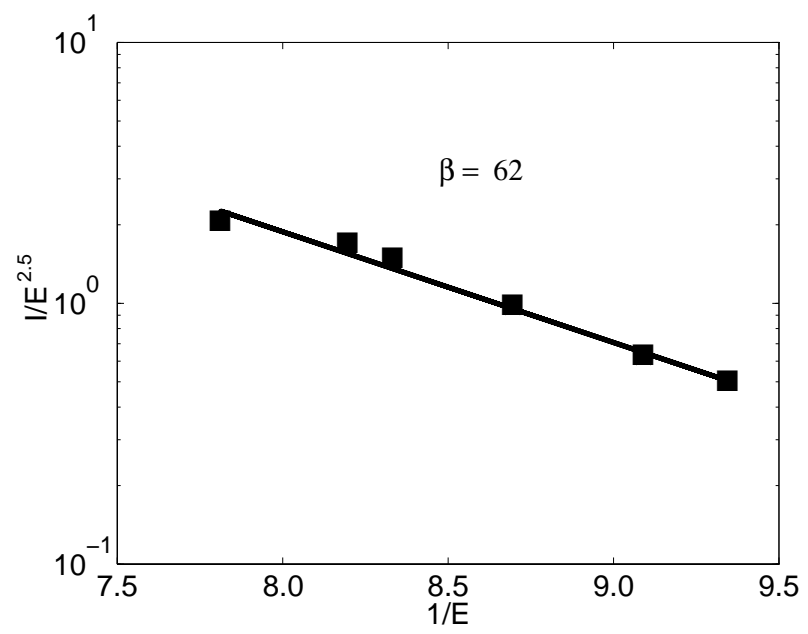

Figure 5.16: Copper cathode normalized Fowler-Nordheim plot.

\begin{tabular}{|l|l|}
\hline Laser Spot Size Hard Edge & $1 \mathrm{~mm}$ radius \\
\hline Cathode Field & $123 \frac{\mathrm{MV}}{\mathrm{m}}$ \\
\hline rf Gun Beam Energy & $5.75 \mathrm{MeV}$ \\
\hline Final Beam Energy & $40 \mathrm{MeV}$ \\
\hline Cathode Magnetic Field & $\leq 4 \mathrm{G}$ \\
\hline
\end{tabular}

Table 5.2: Beam study parameters.

Experimental results indicate that the field emission charge from the 1.6 cell $\mathrm{rf}$ gun utilizing a copper cathode, with a field gradient of $123 \frac{\mathrm{MV}}{\mathrm{m}}$ and a rf macro pulse length of $5.53 \mu \mathrm{sec}$, is approximately $1 \mathrm{nC}$.

\subsection{Transverse Phase Space Emittance Studies}

The normalized transverse rms emittance, $\epsilon_{\mathrm{n}, \mathrm{rms}}$, was measured using the two screen method, discussed in Section 5.3. Table 5.2 lists the relevant beam parameters used during these beam dynamics studies. 


\subsection{1 $\epsilon_{\mathrm{n}, \mathrm{rms}}$ Versus $\mathrm{B}_{\mathrm{z}}$}

The experimental beam dynamics results of $\epsilon_{\mathrm{n}, \mathrm{rms}}$ and $\mathrm{R}_{\mathrm{rms}}$ versus $\mathrm{B}_{\mathrm{z}}$ are shown in Fig. 5.17. $\epsilon_{\mathrm{n}, \mathrm{rms}}$ is the transverse normalized rms emittance, measured using the two screen method discussed in Section 5.3 of Chapter 5. BPM5 and BPM6 were used to measure the rms beam sizes, see Fig. 5.2. $R_{\mathrm{rms}}$ is the rms spot size of the electron beam measured at the HPOP1, which is the first beam profile screen after the second linac section, see Fig. 5.2.

The total electron bunch charge was $0.329 \pm 0.012 \mathrm{nC}$ with an electron bunch length of $\tau_{95 \%}=10.9$ psec. The minimum emittance was found to be $\epsilon_{\mathrm{n}, \mathrm{rms}}=1.17 \pm$ $0.16 \pi \mathrm{mm}$ mrad for a peak magnetic field of $\mathrm{B}_{\mathrm{z}}=1.57 \mathrm{kG}$.

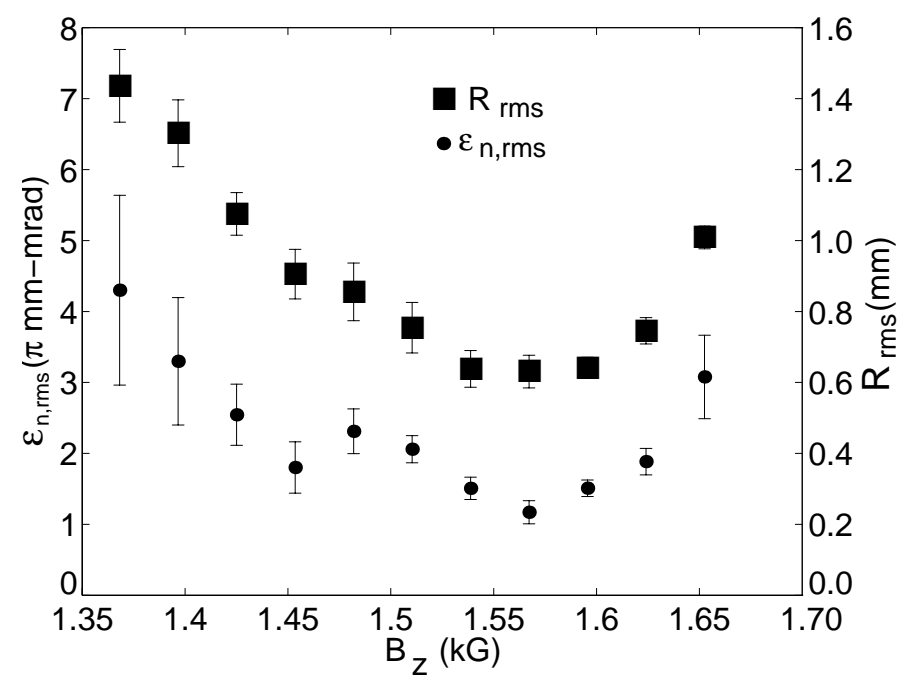

Figure 5.17: Experimental beam dynamics results of $\epsilon_{\mathrm{n}, \mathrm{rms}}$ and $\mathrm{R}_{\mathrm{rms}}$ versus $\mathrm{B}_{\mathrm{z}}$.

The beam line tuning algorithm, used for all of the emittance measurements presented in this dissertation, was to tune the beam transport system that such that the smallest possible symmetric beam profile was produced, $R_{\text {rms }}$ at HPOP1. To tune up the beam line the only elements used were the emittance compensation solenoidal magnet and steering magnets. Subsequent PARMELA [3] simulations indicate that our assumed correlation of minimum emittance and producing the smallest possible symmetric beam profile at HPOP1 was not correct. Fig. 5.18 is a plot of these PARMELA [3] simulations for a total charge of 0.333 nC. These simulations show 
that the beam line should have been tuned to produce a slightly divergent beam at HPOP1. The linac gradient, linac phase, rf/laser phases, rf gun gradient, laser longitudinal and transverse profile were set to the actual experimental conditions. The only beam line parameter that was varied in the PARMELA simulations was the emittance compensation solenoidal magnet field strength.

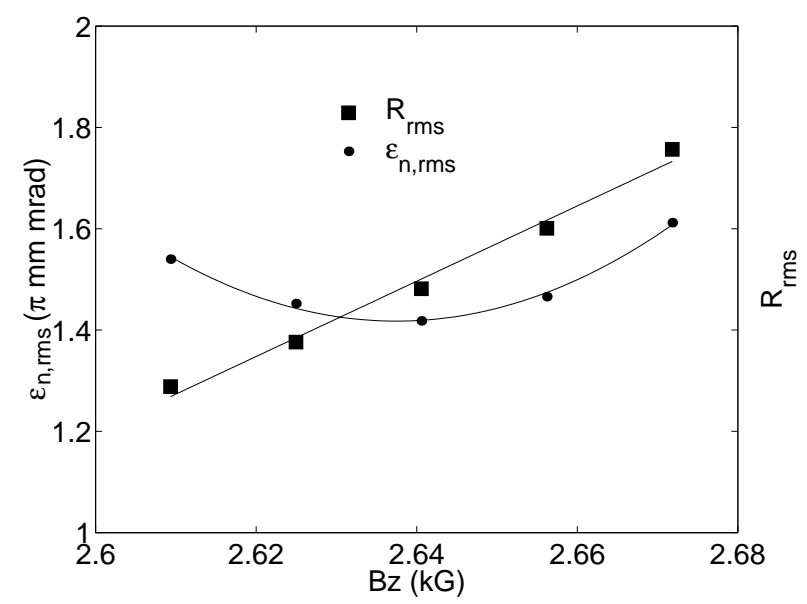

Figure 5.18: PARMELA simulations of $\epsilon_{\mathrm{n}, \mathrm{rms}}$ and $\mathrm{R}_{\mathrm{rms}}$ versus $\mathrm{B}_{\mathrm{z}}$, for a total bunch charge of $0.333 \mathrm{nC}$.

\subsection{2 $\epsilon_{\mathrm{n}, \mathrm{rms}}$ Versus $\mathrm{Q}_{\mathrm{t}}$}

Fig. 5.19 plots in the dependence of transverse normalized rms emittance, $\epsilon_{\mathrm{n}, \mathrm{rms}}$, on the total electron bunch charge, $\mathrm{Q}_{\mathrm{t}}$, for two different beam line tunes. The data for emittance versus charge curve, that has a linear dependence, was taken with a constant solenoidal magnet field that was optimized for a total charge of $390 \mathrm{pC}$ and the laser energy on the cathode was varied. The beam line tune was not changed as the total electron bunch charge was varied.

The data for emittance versus charge, that has a quadratic dependence, was taken after "optimizing the beam line tune" using the solenoid and steering magnets to produce the smallest symmetric beam profile at the first high energy profile screen at each electron bunch charge intensity. Clearly our tuning algorithm increased the 
emittance at lower charges rather than minimizing the emittance. The electron bunch charge was changed by varying the uv laser intensity, as in the first experiment. Even under these diverse experimental conditions, the measured emittances are consistent with the data in Fig. 5.17, in the $0.333 \mathrm{nC}$ regime.

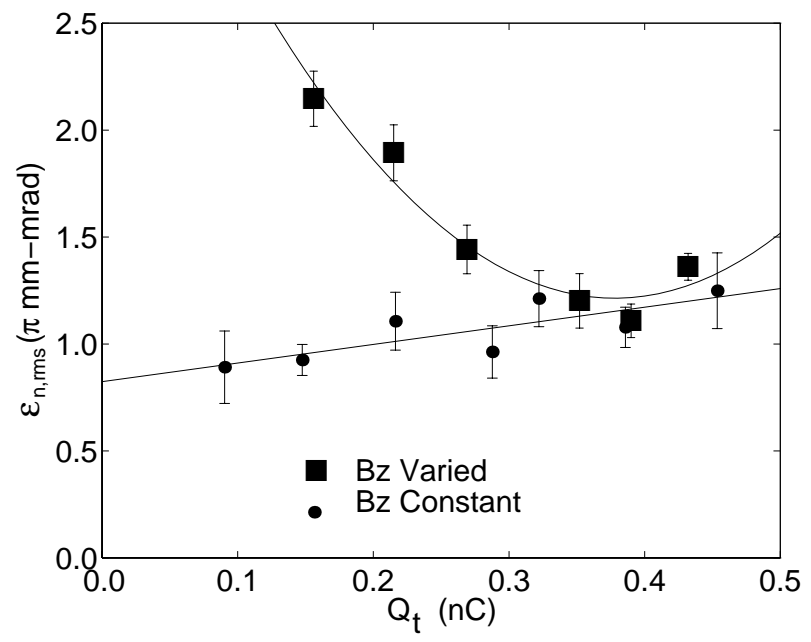

Figure 5.19: Experimental beam dynamics results of $\epsilon_{\mathrm{n}, \mathrm{rms}}$ versus $\mathrm{Q}_{\mathrm{t}}$.

In the limit as the total electron bunch charge goes to zero we see that in the emittance versus charge data, which has a linear dependence, there is a residual emittance term of $0.8 \pi \mathrm{mm}$ mrad. The zero charge emittance is due to $\epsilon_{\mathrm{o}}, \epsilon_{\mathrm{T}}, \epsilon_{\mathrm{rf}}$, $\epsilon_{\mathrm{mp}}$, and $\epsilon_{\mathrm{mag}}$. The space charge emittance term is, $\epsilon_{\mathrm{sc}}=0$, from Eq. 2.22.

$$
\lim _{\mathrm{Q}_{\mathrm{t}} \rightarrow 0} \epsilon_{\mathrm{sc}}=\lim _{\mathrm{I} \rightarrow 0} \frac{\pi}{4} \frac{1}{\alpha \mathrm{k}} \frac{1}{\sin \left(\theta_{\mathrm{o}}\right)} \frac{\mathrm{I}_{\mathrm{p}}}{\mathrm{I}_{\mathrm{A}}} \mu_{\mathrm{x}}(\mathrm{AR})=0
$$

These emittance contribution terms were estimated for this specific beam dynamics experiment in Section 2.3.4. An upper limit estimate of the beam's intrinsic thermal emittance including the Schottky correction is given in Eq. 5.15.

$$
\epsilon_{\mathrm{o}} \leq 0.8 \pm 0.2 \pi \mathrm{mm} \mathrm{mrad}
$$




\subsection{3 $\epsilon_{\mathrm{n}, \mathrm{rms}}$ Versus $\theta_{\mathrm{o}}$}

Due to laser power limitations, rf gun bunch compression, and the Schottky effect it was not possible to keep the total charge constant for different laser injection phases, as was done in our PARMELA simulations in Section 2.4.5 of Chapter 2 where we simulated $\epsilon_{\mathrm{n}, \mathrm{rms}}$ versus $\theta_{\mathrm{o}}$ for a constant charge. Therefore, in Fig. 5.20, the plot of $\epsilon_{\mathrm{n}, \mathrm{rms}}$ as a function of laser injection phase, $\theta_{\mathrm{o}}$, is not for a constant charge but for a decreasing charge from a maximum of $386 \mathrm{pC}$ to a minimum of $76 \mathrm{pC}$.

These results suggest that to attain the smallest possible $\epsilon_{\mathrm{n}, \mathrm{rms}}$ the photoinjector should be operated with the laser injection phase as close as possible to the zero crossing of the rf, but still meet the total electron bunch charge requirement of the experiment. By injecting the laser pulse as close as possible to the zero crossing of the rf, the electron bunch length is decreased and hence the emittance contribution due to the rf fields in the rf gun, which scales as $\epsilon_{\mathrm{rf}} \propto \sigma_{\mathrm{z}}^{2}$, see Eq. 2.27

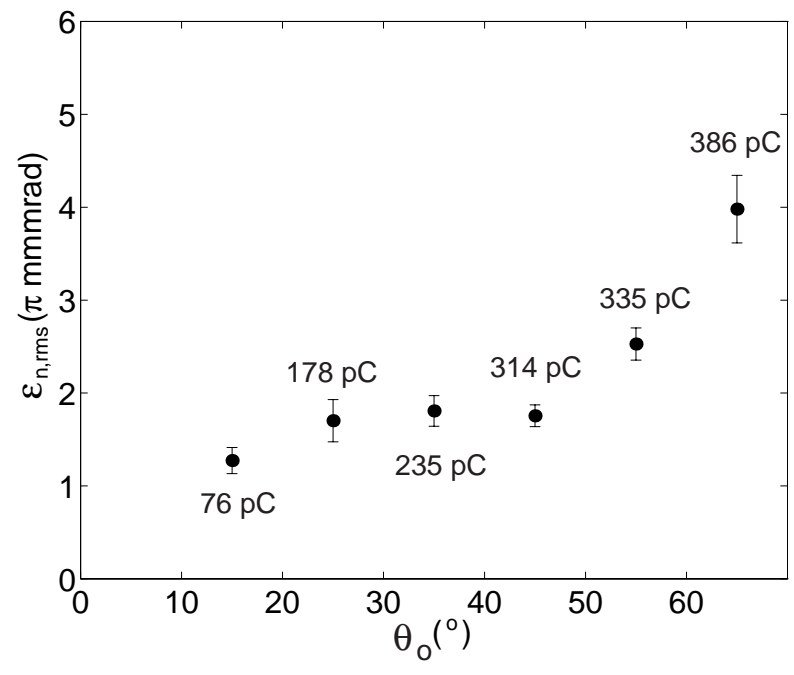

Figure 5.20: $\epsilon_{\mathrm{n}, \mathrm{rms}}$ versus $\theta_{\mathrm{o}}$.

\subsection{Longitudinal Phase Space}

When measuring the bunch length and energy spread of the electron beam, the rf system is adjusted such that the electron bunch initially has a minimum energy 
spread. This is accomplished by adjusting the overall linac phase with respect to the laser injection phase by means of the low level rf system. The $\delta \phi$ between the two linac sections is adjusted by means of a high power rf phase shifter, see Fig. 5.3, such that the energy spread of the beam is minimized. The beam's energy is set to 40 $\mathrm{MeV}$ by adjusting the low level rf drive to the linac klystrons.

\subsubsection{Energy Spread, $\frac{\delta \gamma}{\gamma}$, Versus Linac Phase, $\delta \phi$}

The energy spread, $\frac{\delta \gamma}{\gamma}$, was estimated by measuring the electron beam size on a phosphor screen located downstream of spectrometer magnet in its dispersive region. The dispersion at the beam profile screen is $5.4 \frac{\mathrm{mm}}{\%}$. Fig. 5.21 is a plot of the energy spread of the electron bunch, $\frac{\delta \gamma}{\gamma}$, as a function of the phase difference, $\delta \phi$, between the linac sections for a nominal low level rf/laser injection phase of $45^{\circ}$.

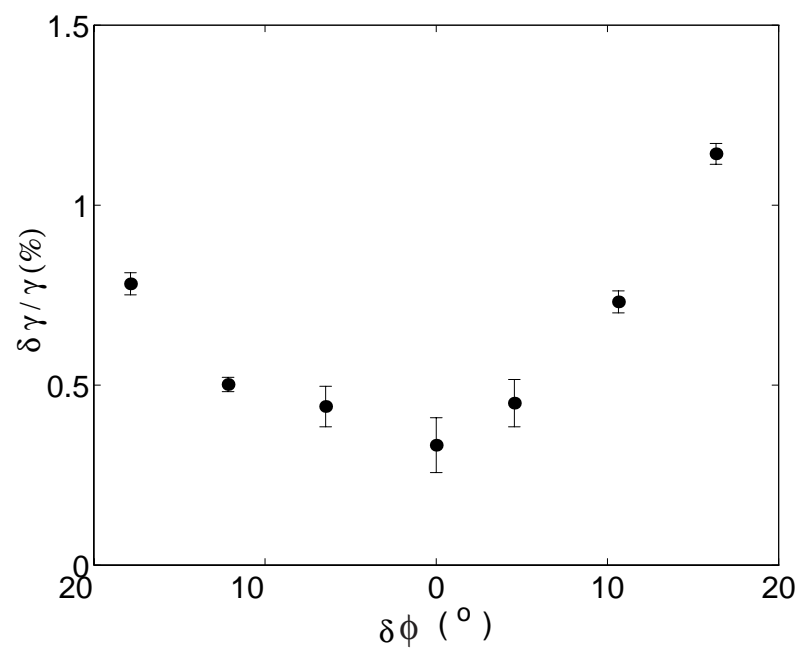

Figure 5.21: RMS energy spread.

\subsubsection{Electron Bunch Length, $\tau_{95 \%}$, Versus Laser Injection Phase, $\theta_{0}$}

Electron bunch length, $\tau_{95 \%}$, versus laser injection phase, $\theta_{\mathrm{o}}$, was measured by de-phasing the second linac section such that a linear energy chirp is produced along 
the bunch [53].

This technique induces a correlation between the longitudinal position in the electron bunch and the energy of the beam. This energy position correlation manifests itself in the spectrometer dispersive region, such that the head of the bunch has a lower energy that the tail.

This energy position correlation technique was used to measure the electron bunch length as a function of laser injection phase. Fig. 5.22 is experimental verification of bunch compression in the 1.6 cell rf gun. This data was taken with a constant laser pulse length of $\tau_{95 \%}=10$ psec, laser energy, and constant solenoidal magnetic field strength. Only the laser injection phases relative to the rf was changed. The Schottky effect therefore decreased the electron bunch charge. Assuming that there is no significant longitudinal debunching due to space charge forces we can produce a map of initial and final phases by numerical solving the coupled differential equation. Bunch compression in the 1.5 cell rf gun, Gun 1B, has been demonstrated previously [54].

$$
\begin{gathered}
\frac{\partial \theta_{\mathrm{o}}}{\partial \mathrm{z}}=\mathrm{k}\left[\frac{\gamma}{\sqrt{\gamma^{2}-1}}-1\right] \\
\frac{\partial \gamma}{\partial \mathrm{z}}=\mathrm{k} \alpha\left[\sin \left(\theta_{\mathrm{o}}\right)+\sin \left(\theta_{\mathrm{o}}+2 \mathrm{kz}\right)\right]
\end{gathered}
$$

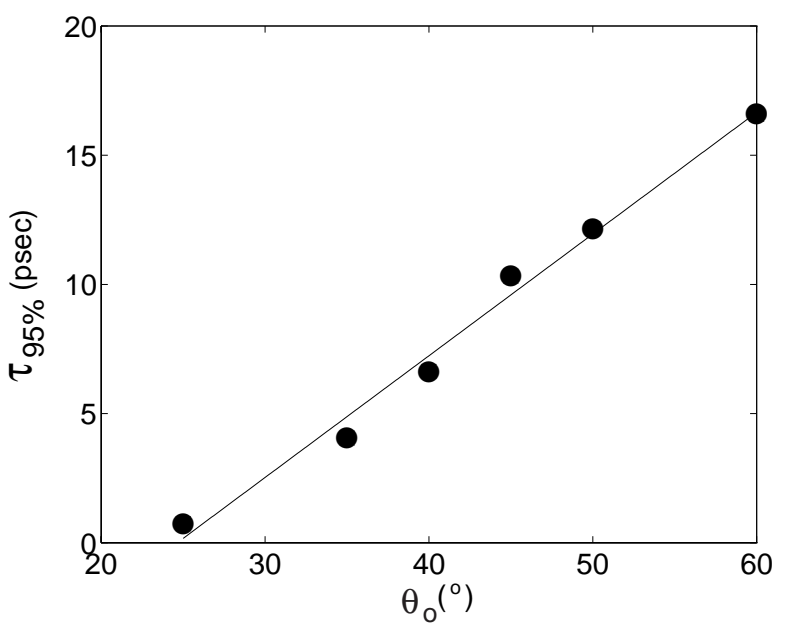

Figure 5.22: $95 \%$ electron bunch length, $\tau_{95 \%}$, as a function of laser injection phase, $\theta_{0}$. 


\subsection{Multi-pole Field Asymmetry}

The symmetry properties of the 1.6 cell rf gun's electro-magnetic fields were probed using eight symmetric electron beam-lets [55]. The electron beam-lets were produced by inserting into the uv laser beam line an eight-fold symmetric aperture. The eightfold aperture produced a uv laser negative of the aperture that struck the photoemitting surface of the rf gun. An image of the uv laser negative is shown in Fig. 5.23. The distance from the center beam-let to the outer ring of beam-lets is $1 \mathrm{~mm}$. The photoemission process then produced the eight symmetric beam-lets used to probe the electro-magnetic fields in the 1.6 cell rf gun. The aperture was rotated to align the symmetry breaking beam-let to be collinear with the waveguide/vacuum port plane.

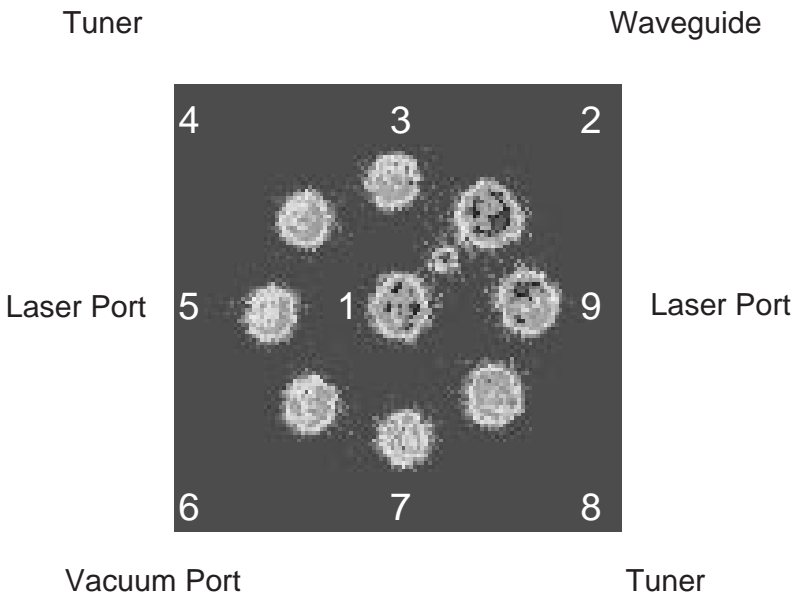

Figure 5.23: Beam-let profile on cathode; the distance from beam-let 1 to beam-lets 2-9 is $1 \mathrm{~mm}$.

\subsubsection{Experimental Setup}

The 1.6 cell rf gun's half cell is symmetrized by two laser ports which are rotated $45^{\circ}$ from the tuner/waveguide coordinate system in the full cell. The input laser port allows for uv light to illuminate the cathode at a grazing incidence angle of $72^{\circ}$ with respect to the cathode surface normal vector. The output laser port prevents multiple scattering of the uv in the half cell of the rf gun after the laser pulse has photoemitted 
the electrons from the cathode. Since the eight electron beam-lets probe both the full and half cell, the data of the electro-magnetic field is an integrated effect. Due to the symmetrized half cell, the integrated electro-magnetic field asymmetry of the 1.6 cell rf gun is only due to the full cell.

A stepping-motor controlled uhv linear motion feed-through with a total travel of $10 \mathrm{~cm}$ is used to position a copper shorting plunger in the vacuum pipe. The copper shorting plunger is used to symmetrize or desymmetrize the 1.6 cell $\mathrm{rf}$ gun. The copper shorting plug does not contact the vacuum pipe wall that forms the cutoff waveguide.

The shorting plunger position for both the symmetrized and desymmetrized mode of operation is shown in Fig 5.24. In the symmetrized case, the shorting plunger is $10.125 \mathrm{~cm}$ from the back wall of the vacuum port iris. The rf power coupled into the vacuum port, which is beyond cutoff, decays exponentially. There is an rf power monitoring loop located in the vacuum pipe which is $-70 \mathrm{~dB}$ down from the input power.

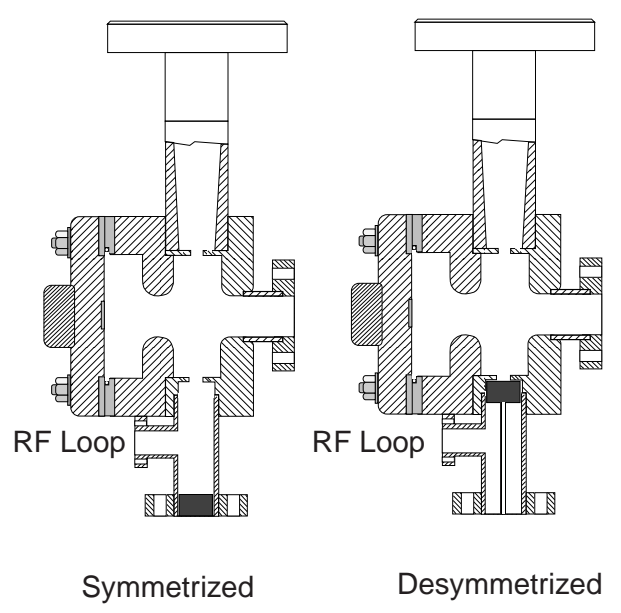

Figure 5.24: rf gun in the symmetrized and desymmetrized mode of operation.

In the desymmetrized mode of operation, the shorting plunger is at the maximum throw of the linear motion feed-through. The rf reflecting surface of the $\mathrm{Cu}$ plunger is $1.25 \mathrm{~mm}$ from the back wall of the full cell to the vacuum port coupling slot, which is $2.25 \mathrm{~mm}$ thick. The vacuum coupling slot is never eliminated and it allows for some 
degree of symmetrization, even in the "desymmetrized" mode of operation.

In the desymmetrized mode of operation the vacuum tube and shorting plunger form a TEM coaxial transmission line. Power is coupled from the full cell into the vacuum tube. During the desymmetrized mode of operation the rf power monitoring loop is $-20 \mathrm{~dB}$ down from the input power.

The TEM coaxial transmission line formed by the vacuum pipe wall and the $\mathrm{Cu}$ shorting plunger along with our inability to completely desymmetrize the full cell did not allow for a direct comparison of our low power rf studies [17] discussed in Section 3.7.2 of Chapter 3. Phase variations in the rf gun, due to breakdown in the TEM coaxial transmission line, prevented emittance measurements from being conducted when the gun is in the desymmetrized mode of operation.

\subsubsection{Experimental Beam Dynamics Studies}

In both modes of operation, the eight electron beam-lets are point to point focused, using the single emittance compensation magnet [56], to a beam-profile monitor located $66.4 \mathrm{~cm}$ from the cathode plane, see Fig. 5.1.

The beam profile in Fig. 5.25 represent the electron beam-let profiles in the symmetric and asymmetric mode of operation with the beam-let 1 located at the geometric center of the rf gun. The betatron rotation of the solenoidal magnet has been removed numerically from these images so that a direct comparison of the individual beam-let positions and distortions with the rf gun's cavity penetrations can be made.
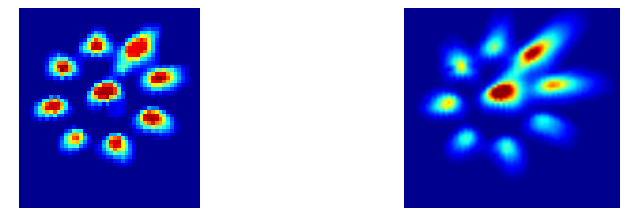

\section{Symmetrized Desymmetrized}

Figure 5.25: Profile of the point to point focused electron beam-lets with rf gun in both the symmetrized and desymmetrized mode of operation.

We have analyzed the eight electron beam-lets in Fig. 5.25 for their Full Width 
Tenth Maximum (FWTM) radial profiles in both the symmetrized and desymmetrized mode of operation of the 1.6 cell rf gun. Fig. 5.26 is a plot of the individual beam-lets FWTM profiles as a function of beam-let number. The beam-let numbers can be correlated with the cavity perturbation using Fig. 5.23.

Qualitatively the data from Fig. 5.26 in the desymmetrized mode of operation, has a dominant dipole mode. In the symmetrized case, there is a noticeable quadrupole mode.

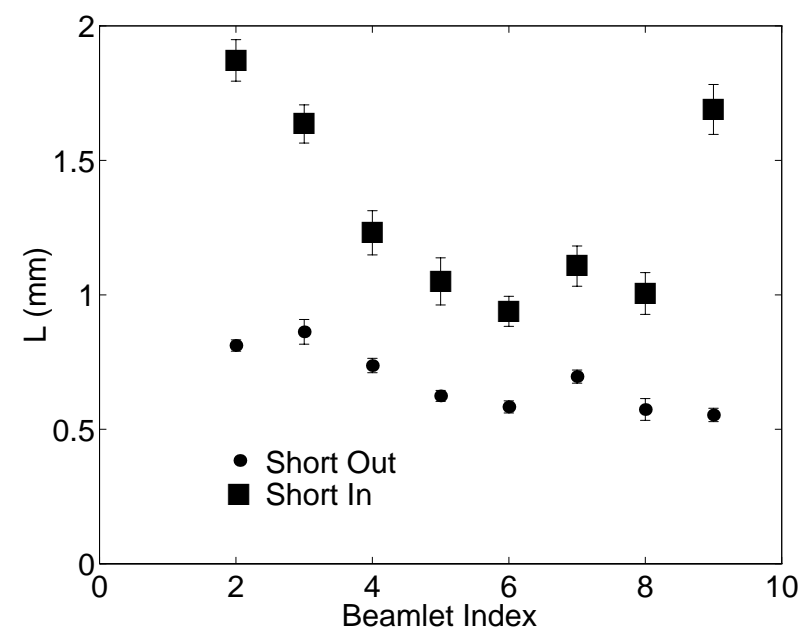

Figure 5.26: Individual beam-let radial extent.

Quantitatively we can fit the data in Fig. 5.27 with a Fourier series as was done in Section 3.7.2, we can see that the dipole mode contribution has been suppressed by a factor of three between the desymmetrized and symmetrized mode of operation.

Laser alignment and dark current studies indicate that the geometrical and integrated electrical centers of the rf gun are within $50 \mu \mathrm{m}$ of each other in the symmetrized mode of operation.

In addition to our beam-lets studies, multi-pole field effects were also studied by decreasing the laser spot size from $2 \mathrm{~mm}$ diameter to $400 \mu \mathrm{m}$ and setting the laser injection phase to the Schottky peak. At this injection phase, the electron bunch length is increased and an energy-spread tail was induced.

By moving the laser spot position on the cathode we were able to minimize the energy spread tail. Using this single electron steering technique, we have minimized the 


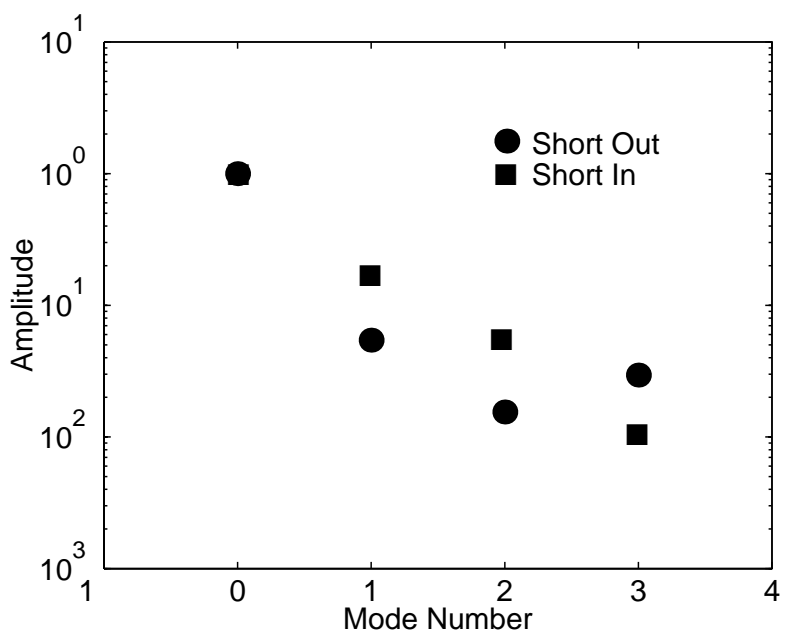

Figure 5.27: Fourier series fit to the data in Fig. 5.26.

beam distortion due to the integrated higher-order-mode contribution of the electromagnetic field. This indicates that the symmetrized BNL/SLAC/UCLA 1.6 cell photocathode rf gun's electrical and geometric centers are within $170 \mu \mathrm{m}$ of each other, which is within the laser spot alignment error of $250 \mu \mathrm{m}$. Compared to similar steering experiments [57] conducted on the 1.5 cell rf gun, Gun 1B, whose electrical and geometric centers differ by $1.0 \mathrm{~mm}$, the 1.6 cell gun has fulfilled the symmetrization criteria of its design.

\subsection{Cathode Magnetic Field Asymmetry}

In this section we present our experimental beam dynamics studies of the effects of a finite longitudinal magnetic field at the cathode. Three effects were studied: the relative angular rotation due to the cathode magnetic field; the rms spot size; and the normalized rms emittance due to this magnetic field.

From Busch's Theorem [28] we know that

$$
\dot{\theta}=\frac{\mathrm{e}}{2 \gamma \mathrm{m}_{\mathrm{o}}}\left(\mathrm{B}_{\mathrm{z}_{\mathrm{o}}}-\mathrm{B}_{\mathrm{z}}\right)
$$

where $\mathrm{e}$ is the charge of an electron, $\mathrm{m}_{\mathrm{o}}$ is the electron rest mass, $\gamma$ is the relativistic 
correction factor defined in Eq. 2.2, $\mathrm{B}_{\mathrm{z}_{\mathrm{o}}}$ is the cathode magnetic field, and $\mathrm{B}_{\mathrm{z}}$ is the longitudinal magnetic field downstream of the cathode at a position $\mathrm{z}$.

Integrating Eq. 5.18, we find that the angular rotation of the electron beam in a field free region where $B_{z}=0$, varies linearly with the cathode magnetic field.

$$
\delta \theta=\int \frac{\mathrm{e}}{2 \beta \gamma \mathrm{m}_{\mathrm{o}} \mathrm{c}} \mathrm{dz} \mathrm{B}_{\mathrm{z}_{\mathrm{o}}}
$$

The change in the rms spot size can be calculated using Eq. 5.20, which is used to define $\mathrm{x}_{\mathrm{rms}}^{\prime}$.

$$
\mathrm{x}_{\mathrm{rms}}^{\prime} \equiv \frac{\mathrm{p}_{\mathrm{x}, \mathrm{rms}}}{\mathrm{p}_{\mathrm{z}}}=\frac{\mathrm{v}_{\mathrm{x}, \mathrm{rms}}}{\beta \mathrm{c}}
$$

The transverse velocity, defined in Eq. 5.21, can be calculated by inserting the value of $\dot{\theta}$ from Busch's Theorem.

$$
\mathrm{v}_{\mathrm{x}, \mathrm{rms}} \equiv \sqrt{\frac{\iint \mathrm{r}^{2} \dot{\theta}^{2} \cos ^{2}(\theta) \mathrm{rdrd} \theta}{\iint \mathrm{rdrd} \theta}}
$$

This gives the result that the change in the transverse rms spot size scales as $\left|B_{z_{o}}\right|$, Eq. 5.22 .

$$
\delta \mathrm{x}_{\mathrm{rms}}=\int \mathrm{x}_{\mathrm{rms}}^{\prime} \mathrm{dz}=\int \frac{\mathrm{eR}_{\mathrm{o}}}{4 \beta \gamma \mathrm{m}_{\mathrm{o}} \mathrm{c}} \mathrm{dz}\left|\mathrm{B}_{\mathrm{z}_{\mathrm{o}}}\right| .
$$

In Section 2.3.3 of Chapter 2, we found that the magnetic emittance scales as $\epsilon_{\mathrm{B}_{\mathrm{z}}} \propto \mathrm{R}_{\mathrm{o}}^{2}\left|\mathrm{~B}_{\mathrm{z}_{\mathrm{o}}}\right|$. This result is repeated in Eq. 5.23 for completeness.

$$
\epsilon_{\mathrm{n}, \mathrm{rms}}=\beta \gamma \sqrt{<\mathrm{x}^{\prime 2}><\mathrm{x}^{2}>}=\frac{\mathrm{eR}_{\mathrm{o}}^{2}\left|\mathrm{~B}_{\mathrm{z}_{\mathrm{o}}}\right|}{8 \mathrm{~m}_{\mathrm{o}} \mathrm{c}}
$$

\subsubsection{Experimental Setup}

A variable cathode magnetic field, not dependent on the emittance compensation magnet field strength, was induced by the bucking magnet shown in Fig 5.1. Precise control of the bucking magnet current was providing by a uni-polar DC power supply and a shunt resistor. Since a bi-polar power supply was not available the current leads 
had to be reversed to change the sign of the cathode magnetic field. Magnetic field measurements of the bucking magnet were presented in Section 4.4.1 of Chapter 4 .

\subsubsection{Experimental Beam Dynamics Studies}

Due to the small but finite field at the rf gun cathode, the electron bunch is produced with a finite angular momentum. We measured the relative angular rotation due to this finite cathode magnetic field. In this experiment an eight-fold symmetric mask [55] was inserted into the the laser beam, thereby producing the laser profile on the cathode shown in Fig. 5.28. The smallest beam-let located at $45^{\circ}$ is used to break the symmetry. In this way we were able to measure the betatron rotation of the beam through the solenoidal magnet which was found to be approximately $90^{\circ}$.

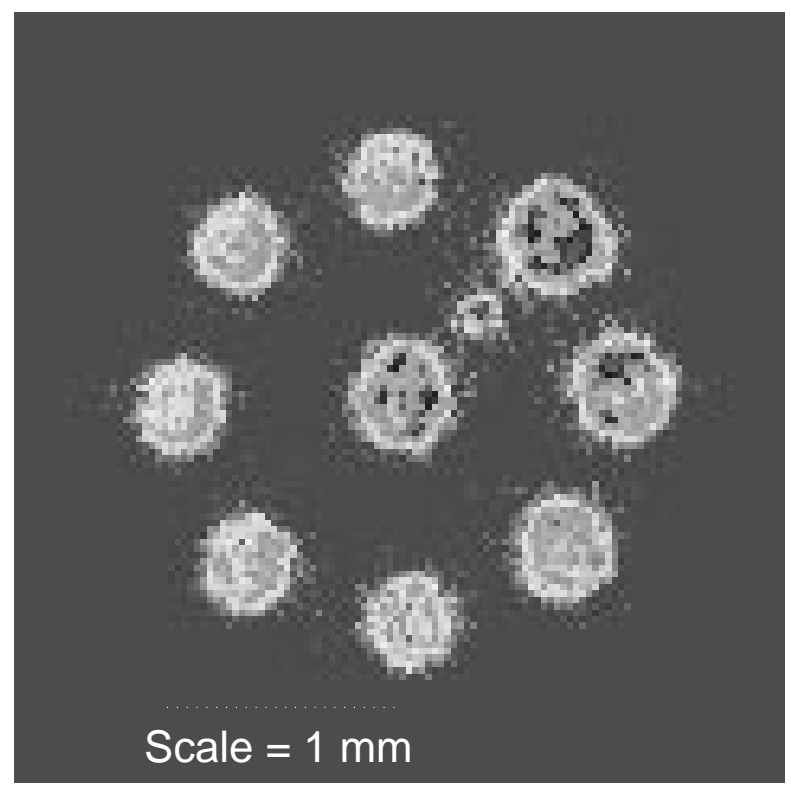

Figure 5.28: Eight-fold symmetric beam-lets.

By using point-to-point imaging of the eight-fold electron beam-lets from the cathode to a beam profile monitor located $66.4 \mathrm{~cm}$ from the cathode plane, we measured the relative angular rotation of the electron beam as a function of the induced cathode magnet field. Fig. 5.29 represents the relative angular rotation of the electron 
beam-lets due to the cathode magnetic field and has a linear dependence as predicted in Eq. 5.19.

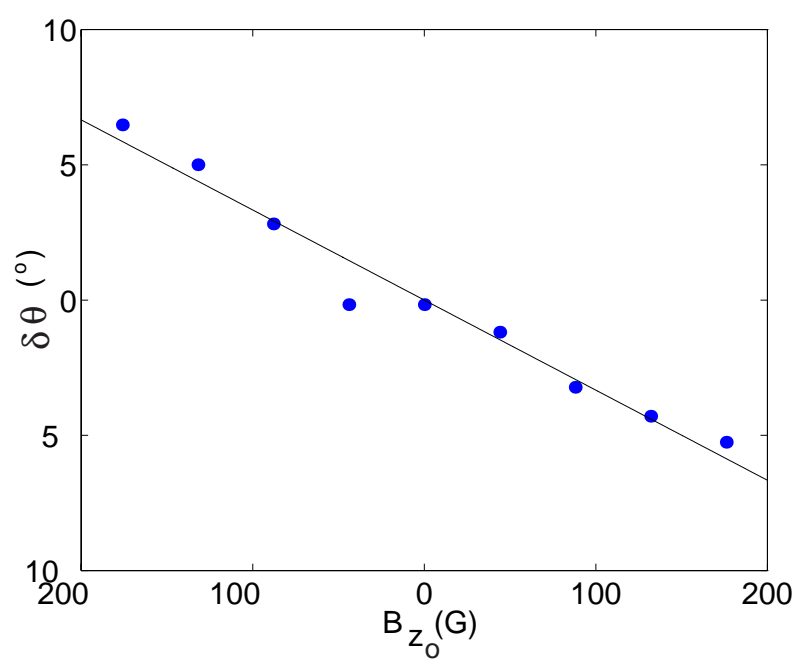

Figure 5.29: $\delta \theta$ versus $\mathrm{B}_{\mathrm{z}_{\mathrm{o}}}$.

The rms spot size as a function of the cathode magnet field was measured at a beam profile screen located at the output of the second linac section. The energy of the electron beam was $40 \mathrm{MeV}$. The functional dependence of the high energy spot size data in Fig. 5.30 was fit to Eq. 5.22 with a minimum spot size occurring with a cathode magnetic field of $-5 \mathrm{G}$. The cathode field with the bucking magnet turned off is $+4 \mathrm{G}$. This indicates that the absolute value of the cathode field cannot be zeroed better than that provided by the mechanical construction and self-alignment of NGP.

Due to rf/laser injection phase instabilities it was not possible to measure the emittance growth due to the induced cathode magnetic field. From the spot size variation at $40 \mathrm{MeV}$, due to the induced cathode magnetic field, and the correlation between the measured spot size and $\epsilon_{\mathrm{n}, \mathrm{rms}}$ [58], the emittance growth as a function of the induced cathode magnetic field was estimated. In Table 5.3 the inferred experimental results of $\epsilon_{\mathrm{n}, \mathrm{rms}}$ growth as a function of an induced cathode magnetic field are compared with PARMELA [3] simulation and theoretical predictions from Section 2.3.3 of Chapter 2. 


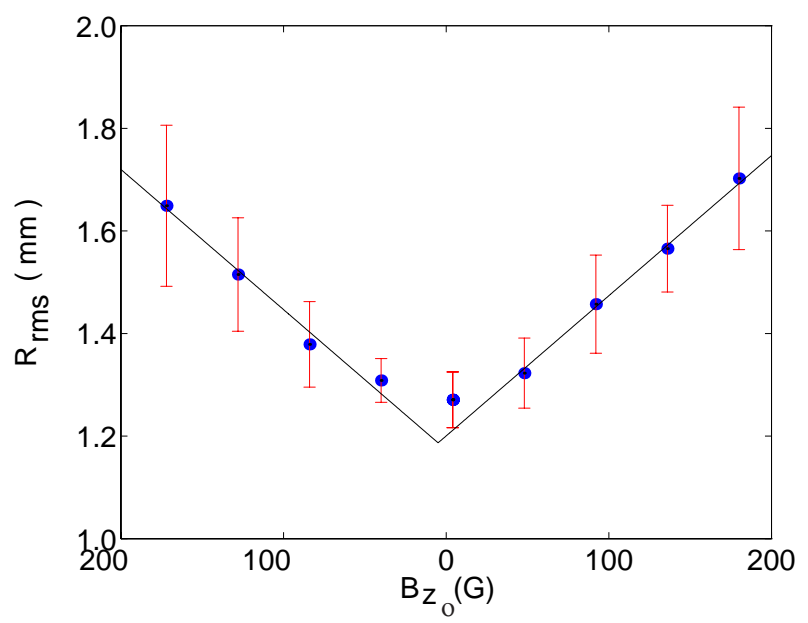

Figure 5.30: High energy $R_{r m s}$ versus $B_{z(z=0)}$.

\begin{tabular}{|l|l|}
\hline Experimental Result & $0.010 \frac{\pi \mathrm{mm} \mathrm{mrad}}{\mathrm{G}}$ \\
\hline PARMELA & $0.006 \frac{\pi \mathrm{mm} \mathrm{mad}}{\mathrm{G}}$ \\
\hline THEORY & $0.007 \frac{\pi \mathrm{mmad}}{\mathrm{G}}$ \\
\hline
\end{tabular}

Table 5.3: Comparison of inferred experimental results, PARMELA simulation, and theory for the emittance growth due to the cathode magnetic field. 


\begin{tabular}{|l|l|l|}
\hline Parameter & LCLS & Experimental Results \\
\hline $\mathrm{Q}_{t}$ & $1 \mathrm{nC}$ & $1.020 \pm 0.059 \mathrm{nC}$ \\
\hline$\tau_{95 \%}$ & $10 \mathrm{psec}$ & $14.7 \mathrm{psec}$ \\
\hline$\epsilon_{\mathrm{n}, \mathrm{rms}}$ & $1.00 \pi \mathrm{mm} \mathrm{mrad}$ & $4.74 \pm 0.24 \pi \mathrm{mm} \mathrm{mrad}$ \\
\hline
\end{tabular}

Table 5.4: $1 \mathrm{nC}$ emittance results.

\subsection{1 nC Emittance Studies}

In a SASE x-ray FELs, such as the LCLS, the relevant photoinjector parameters are $\mathrm{Q}_{\mathrm{t}}=1 \mathrm{nC}, \tau_{95 \%}=10 \mathrm{psec}$, and $\epsilon_{\mathrm{n}, \mathrm{rms}} \leq 1.00 \pi \mathrm{mm} \operatorname{mrad}$. Using the NGP, these beam parameters have been experimentally measured and are compared to the LCLS beam requirements in Table 5.4.

Simulation results using PARMELA [3] with a longitudinal flat-top electron pulse of $\tau_{95 \%}=10$ psec, FWHM, indicate that $\epsilon_{\mathrm{n}, \mathrm{rms}} \leq 1 \pi \mathrm{mm} \operatorname{mrad}$ is attainable [18].

The results of our experimental beam dynamics studies present two experimental issues that need to be investigated in the future. First, is the increase in the electron bunch length, $\tau_{95 \%}$, and the second is the larger than expected $\epsilon_{\mathrm{n}, \mathrm{rms}}$. These issues are not unrelated, since the emittance contribution due to $\mathrm{rf}$ scales as the electron bunch length squared, $\sigma_{\mathrm{z}}^{2}$.

Two possible bunch lengthening mechanisms were studied: laser pulse bunch lengthening due to laser power saturation in the second doubling crystal, and bunch lengthening due to the longitudinal space charge force in the electron bunch.

Fig. 5.31 depicts the results of our experimental investigation of the correlation between the laser power, $\mathrm{E}_{\gamma}$, and the electron bunch length, $\tau_{95 \%}$. While keeping the total electron bunch charge constant at $360 \mathrm{pC}$, the laser intensity in the green $(\lambda=532 \mathrm{~nm})$ was increased. The result is that the electron bunch length is a constant, $\tau_{95 \%}=10.9$ psec, as is plotted in Fig. 5.31.

Longitudinal space charge de-bunching was the second mechanism we studied. The laser intensity in the green was kept a constant $3 \mathrm{~mJ}$. By varying the uv laser intensity, the electron bunch charge was increased from $400 \mathrm{pC}$ to $1 \mathrm{nC}$. This resulted in an increase in the electron bunch length, $\tau_{95 \%}$, from 10.9 psec to 14.7 psec, see 
Fig. 5.31.

The results of these beam dynamics experiments show that the $1 \mathrm{nC}$ electron bunch lengthening is not a laser saturation effect, but, rather it is due to an increase in the longitudinal space charge forces in the electron beam.

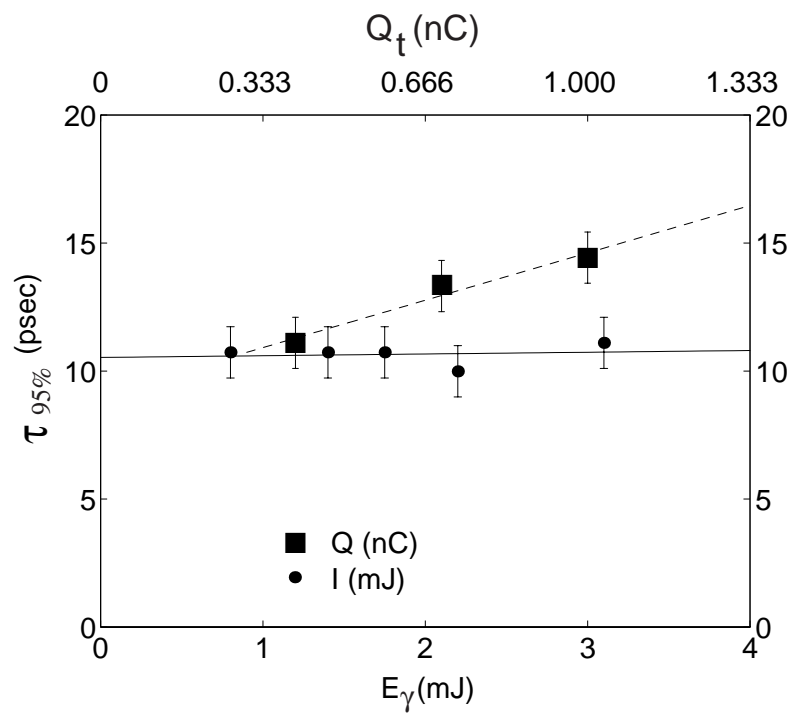

Figure 5.31: Bunch length, $\tau_{95 \%}$, versus green laser intensity, $\mathrm{E}_{\gamma}$, and total electron bunch charge, $Q_{\mathrm{t}}$.

Another factor that causes transverse emittance growth is that due to the nonuniformity of the transverse laser pulse and transverse variation of the cathode quantum efficiency, the electron bunch transverse charge density $\rho(\mathrm{x}, \mathrm{y})$ will not be uniform. This degrades the first order linear space charge emittance compensation process.

Laser assisted explosive electron emission (LAEEE) [51] was used to smooth out these nonlinear space charge effects. The measured improvements in $\epsilon_{\mathrm{n}, \mathrm{rms}}$ due to LAEEE are presented in Table 5.5. These results are not statistically significant. But the total time allowed for LAEEE cleaning was severely limited due to beam time constraints. Qualitative data for $\mathrm{QE}(\mathrm{x}, \mathrm{y})$ of the copper cathode is not available, but for the magnesium cathode, LAEEE improved the $\mathrm{QE}(\mathrm{x}, \mathrm{y})$ by a factor of two, from 


\begin{tabular}{|l|l|}
\hline \multicolumn{2}{|c|}{ Before Laser Cleaning } \\
\hline $\mathrm{Q}_{\mathrm{t}}$ & $918 \pm 77.5 \mathrm{pC}$ \\
\hline$\epsilon_{\mathrm{n}, \mathrm{rms}}$ & $5.18 \pm 0.25 \pi \mathrm{mm} \mathrm{mrad}$ \\
\hline \multicolumn{2}{|c|}{ After Laser Cleaning } \\
\hline $\mathrm{Q}_{\mathrm{t}}$ & $1020 \pm 58.7 \mathrm{pC}$ \\
\hline$\epsilon_{\mathrm{n}, \mathrm{rms}}$ & $4.74 \pm 0.24 \pi \mathrm{mm} \mathrm{mrad}$ \\
\hline
\end{tabular}

Table 5.5: High charge emittance study results before and after LAEEE.

an order of magnitude variation across the cathode spot to $50 \%$ after LAEEE.

\subsection{Summary}

\subsubsection{Quantum Efficiency Studies}

Quantum efficiency studies of OFHC grade II $\mathrm{Cu}$ and $99.8 \% \mathrm{Mg}$ cathode plug have been studied. Data of total electron bunch charge versus $\Omega_{\gamma}$ determined the setting of the laser polarization. $\Omega_{\gamma}$ was set to $61.5^{\circ}$ and $65.0^{\circ}$ for $\mathrm{Cu}$ and $\mathrm{Mg}$, respectively. These settings maximized the total available charge for a constant laser energy. The variation in the lasers relative polarization could be due the cathode plate orientation and/or the crystal structure of the photocathode material. In either case, the lasers polarization charge should be adjusted to maximize the total available charge from the newly replaced cathode plate. Using Eq. 5.11, the QE of $\mathrm{Cu}$ and $\mathrm{Mg}$ was determined to be $\mathrm{QE}=4.5 \times 10^{-5}$ and $\mathrm{QE}=3.55 \times 10^{-4}$ for $\mathrm{Cu}$ and $\mathrm{Mg}$, respectively. In addition, the 2-D variation of the photoemitting surfaces was studied using a laser scanning technique. The QE variation of photocathode was found to be $25 \%$ and $50 \%$ for $\mathrm{Cu}$ and $\mathrm{Mg}$, respectively.

\subsubsection{Energy Studies}

The maximum electron beam energy, $\gamma \mathrm{m}_{\mathrm{o}} \mathrm{c}^{2}$, for an accelerating gradient of $123 \frac{\mathrm{MV}}{\mathrm{m}}$ was $5.75 \mathrm{MeV}$. A Fowler-Nordheim plot indicates that the field enhancement factor of the 1.6 cell $\mathrm{rf}$ gun is $\beta=62$. The 1.6 cell $\mathrm{rf}$ gun produces approximately $1 \mathrm{nC}$ of 
dark current with a field gradient of $123 \frac{\mathrm{MV}}{\mathrm{m}}$ and a rf macro pulse length of $5.53 \mu$ sec, for a $\mathrm{Cu}$ cathode.

The 1.6 cell rf gun was able to operate with a maximum field gradient of $150 \frac{\mathrm{MV}}{\mathrm{m}}$ with a rf macro pulse length of $0.70 \mu \mathrm{sec}$. Further conditioning would have raised the maximum field level that the 1.6 cell rf gun could hold off without excessive breakdown, but this was not attempted due to operational requirements of the ATF. The 1.6 cell rf gun, with a $\mathrm{Cu}$ cathode, is normally operated with a field gradient of $123 \frac{\mathrm{MV}}{\mathrm{m}}$.

\subsubsection{Transverse Normalized rms Emittance Studies}

The transverse normalized emittance, $\epsilon_{\mathrm{n}, \mathrm{rms}}$, was measured as a function of compensation magnetic field strength, $B_{z}$, total electron bunch charge, $Q_{t}$, and laser injection phase, $\theta_{0}$.

The minimum $\epsilon_{\mathrm{n}, \mathrm{rms}}$ measured as a function of $\mathrm{B}_{\mathrm{z}}$ was $1.17 \pm 0.16 \pi \mathrm{mm} \mathrm{mrad}$, for an electron bunch charge of $0.329 \pm 0.012 \mathrm{nC}$ and a FWTH electron bunch length of $\tau_{95 \%}=10.9$ psec. The peak magnetic field needed to attain these beam parameters was $\mathrm{B}_{\mathrm{z}}=1.57 \mathrm{kG}$.

The dependence of $\epsilon_{\mathrm{n}, \mathrm{rms}}$ versus $\mathrm{Q}_{\mathrm{t}}$ was measured for two different beam line tunes. Even under these diverse experimental conditions, the measured emittances are consistent with the data from the minimum $\epsilon_{\mathrm{n}, \mathrm{rms}}$ attained in the emittance compensation field strength scans.

The linearly dependent data from the $\epsilon_{\mathrm{n}, \mathrm{rms}}$ versus $\mathrm{Q}_{\mathrm{t}}$ scan in the limit of zero charge determined the upper limit of the thermal emittance of the electron beam, $\epsilon_{\mathrm{o}} \leq 0.8 \pm 0.2 \pi \mathrm{mm} \mathrm{mrad}$

$\epsilon_{\mathrm{n}, \mathrm{rms}}$ versus $\theta_{\mathrm{o}}$ suggest that to attain the smallest possible $\epsilon_{\mathrm{n}, \mathrm{rms}}$, the photoinjector should be operated with the laser injection phase as close as possible to the zero crossing of the rf. 


\subsubsection{Longitudinal Phase Space}

The electron beam energy was measured to be $40 \mathrm{MeV}$ with an energy spread of

$\frac{\delta \gamma}{\gamma} \leq 0.5 \%$. The electron bunch length, $\tau_{95 \%}$, was measured in the low charge regime as a function of the laser injection phase, $\theta_{0}$.

\subsubsection{Multi-Pole Field Studies}

The symmetry properties of the 1.6 cell rf gun's electro-magnetic fields were probed by three different experimental techniques.

The first technique utilized eight symmetric electron beam-lets produced by inserting a metallic aperture into the uv laser transport line. The FWTM radial profiles of each beam-let was measured and their variation was analyzed using Fourier transform theory. A factor of three reduction in the dipole mode asymmetry of the longitudinal accelerating field was found between the symmetrized and desymmetrized mode of operation.

Laser alignment and dark current studies indicate that the geometrical and integrated electrical centers of the rf gun are within $50 \mu \mathrm{m}$ of each other in the symmetrized mode of operation.

Multi-pole field effects were also studied by decreasing the laser spot size from 2 mm diameter to $400 \mu \mathrm{m}$ and setting the laser injection phase to the Schottky peak. At this injection phase electron bunch length is increased and an energy-spread tail was induced.

By moving the laser spot position on the cathode, we were able to minimize the energy spread tail. Using this single electron steering technique, we were able to minimize the beam distortion due to the integrated higher-order-mode contribution of the electro-magnetic field. This indicates that the symmetrized BNL/SLAC/UCLA 1.6 cell photocathode rf gun's electrical and geometric centers are within $170 \mu \mathrm{m}$ of each other, which is within the laser spot alignment error of $250 \mu \mathrm{m}$. Compared to similar steering experiments [57] conducted on the 1.5 cell rf gun, Gun 1B, whose electrical and geometric centers differ by $1.0 \mathrm{~mm}$, the 1.6 cell gun has fulfilled the symmetrization criteria of its design. 


\subsubsection{Cathode Magnetic Field Studies}

Beam dynamics experiments were conducted to determine the effect that our single emittance compensation magnet would have on the electron beam performance.

The relative angular rotation, $\delta \theta$, of the electron beam along with the change in the rms spot size, $\delta \mathrm{x}_{\mathrm{rms}}$, was measured as a function of the induced cathode magnet field.

Busch's theorem predicts that a linear relationship between $\delta \theta$ and the induced cathode magnetic exists. This was verified through our experimentation.

A minimum in $\delta \mathrm{x}_{\mathrm{rms}}$ occurred with a cathode magnetic field of $-5 \mathrm{G}$. Magnetic measurements of the single emittance compensation magnet indicate that the cathode field during normal operation is $+4 \mathrm{G}$. These results suggest that the absolute value of the cathode magnetic field cannot be zeroed with this technique better than what is provided by the mechanical construction and self-alignment of NGP.

Correlating $\delta \mathrm{x}_{\mathrm{rms}}$ with $\epsilon_{\mathrm{n}, \mathrm{rms}}$, the emittance growth as a function of the induced cathode magnetic field was determined to scale as $0.010 \frac{\pi \mathrm{mm} \mathrm{mrad}}{\mathrm{G}}$.

\subsubsection{1 nC Transverse Normalized rms Emittance Studies}

In the high charge regime, $\mathrm{Q}_{\mathrm{t}}=1 \mathrm{nC}$, the normalized rms emittance, $\epsilon_{\mathrm{n}, \mathrm{rms}}$ was measured to be $4.74 \pm 0.24 \pi \mathrm{mm}$ mrad, for an electron bunch length of 14.7 psec.

Two possible bunch lengthening mechanisms were studied. No correlation between the laser energy (green) and $\tau_{95 \%}$ for a constant electron bunch charge of $360 \mathrm{pC}$ was found. On the other hand, a linear correlation between the electron bunch length and charge was found. An increase in the electron bunch length from 10.9 psec to 14.7 psec was measured as the total electron bunch charge was increased from $400 \mathrm{pC}$ to $1 \mathrm{nC}$. The laser injection phase was held constant along the green laser intensity for this experiment. 
Chapter 6

Conclusions and Future Work 


\subsection{Conclusions}

The ultimate goal of the Next Generation Photoinjector project was to produce a $1 \mathrm{nC}$ electron beam with a transverse normalized rms emittance of less than $1 \pi$ $\mathrm{mm}$ mrad. This was not accomplished. The best emittance attained at the $1 \mathrm{nC}$ level was $\epsilon_{\mathrm{n}, \mathrm{rms}}=4.74 \pm 0.24 \pi \mathrm{mm}$ mrad, using a longitudinal Gaussian laser pulse of FWHM $=10.5$ psec. The measured electron bunch length was 14.7 psec. When compared to our low charge electron bunch length of 10.5 psec, clearly longitudinal space charge forces have become a dominate effect in the $1 \mathrm{nC}$ charge regime. The 1.6 cell rf gun normally operates with a rf field gradient of $123 \frac{\mathrm{MV}}{\mathrm{m}}$. This field level was slightly lower than the optimal field gradient predicted by PARMELA. This was due to operational requirements of the ATF rather than any problem inherent in the 1.6 cell rf gun itself. The suppression of the transverse dipole mode in the rf gun was successful. No significant emittance growth was noted due to the single emittance compensation magnet.

Although the NGP did not meet all of its design goals, our research has had the beneficial effect on the accelerator physics community of directing future research with the objective of reaching the $1 \pi \mathrm{mm}$ mrad goal. In the remainder of this chapter, we shall point out what we believe are the areas of research necessary to attain the 1 $\pi \mathrm{mm}$ mrad, $1 \mathrm{nC}$ goal. For more detailed conclusions and a summary of results, the reader is directed to the conclusion section at the end of each chapter.

\subsection{Future Work}

The results of our beam dynamics simulation, low power radio-frequency measurements, emittance compensation magnet design, and experimental beam dynamics studies point the way for future work in the area of emittance compensated rf photoinjectors. Here we present a coherent research plan that will allow for the elucidation and development of high brightness electron sources that will meet or exceed the Linear Coherent Light Sources (LCLS) and/or the Next Linear Collider (NLC) injector requirements. 


\subsubsection{Beam Dynamics Simulations}

In Chapter 2, we have taken the first steps to fully mapping the n-dimensional operational parameter space of the Next Generation Photoinjector (NGP). Using theoretical prediction of Serafini and Rosenzweig [4], a systematic study of the NGP operational parameter space should be undertaken. This study should not use the method of least descent, but should look at the correlation function between all of the parameters of the operational space. This will entail producing a $1 \times \mathrm{m} \times \mathrm{n}$ matrix array of emittance values and finding the minimum in the n-dimensional operational parameter space, where $1 \mathrm{x} \mathrm{m}$ is the dimensionality of the $\mathrm{i}^{\text {th }}$ parameter studied of the n-dimensional operational parameter space.

The validity of space charge dominated beam dynamics codes is critical for the success of a photoinjector design. A group was formed at PAC97 to cross-check the validity of different space charge dominated beam dynamics codes with respect to experimental results of the BNL/SLAC/UCLA 1.6 cell S-band emittance compensated rf gun [59]. This work should be given the highest priority.

\subsubsection{Magnet and rf Design}

In Chapter 4 we pointed out that the single emittance compensation solenoidal magnet could not be positioned in the optimal position for emittance compensation due to mechanical interference of the waveguide feed into the full cell. To maximize the performance of future emittance compensated photoinjectors, this mechanical design constraint must be eliminated.

Work in this area is currently ongoing at the Deutsches Elektronen-Synchrotron (DESY) with the 1.5 cell L-band coaxial rf gun. This type of rf power feed allows for positing the compensation magnet around the rf gun body at the optimal position. But since the bore of the emittance compensation magnet will have an inner diameter that is at least as large as the outer diameter of the rf gun body, there would be a significant cathode magnetic field if a single compensation magnet design were implemented. Consequently, a bucking magnet will need to be installed upstream of the cathode plane to mitigate the emittance growth term due to the cathode magnetic 
field.

Due to its multi-cell design, the Plane Wave Transformer (PWT) rf gun under development at UCLA has the advantage of requiring a lower rf field level than the 1.6 cell rf gun to attain the transverse emittances required for the LCLS. The multicell design also moves the rf feed into the rf gun away from the cathode region, thereby eliminating the mechanical constraint that limited the performance of the 1.6 cell rf gun. The PWT rf gun is an open structure such that its pumping speed in the cathode region is much larger than that of the 1.6 cell $\mathrm{rf}$ gun. As a result, vacuum levels needed for spin polarized electron production might be attained.

\subsubsection{Experimental Issues}

\section{Quantum Efficiency}

In Section 5.4.2, we have shown that in the best case the 2-D quantum efficiency of our cathode had a variation of $25 \%$ over the photo-emitting area of the photocathode material. Simulations have shown that this will lead to a $50 \%$ growth in the beam's transverse emittance [19]. This variation must be corrected to ensure that the electron beam's transverse charge distribution is optimal for the emittance compensation process.

A correction scheme for the cathode QE variation problem has been suggested [60]. By measuring the energy variation of the transverse laser profile with a calibrated CCD and the transverse electron charge distribution with an multi-pole strip line, a correction signal can be sent to an octagonal array of liquid crystal pixels, which is an active element in the laser transport line. This allows for the control of the laser's transverse energy distribution. When the laser energy distribution is convolved with the 2-D QE of the photocathode material, it will produce an electron charge distribution that is optimized for the emittance compensation process. This noninvasive feedback system is presented in Fig. 6.1.

Using another CCD to measure the 2-D variation of the electron beam transverse profile as measured on a beam profile screen, we could eliminate the need for the feedback control system. This method would be invasive and would require periodic 


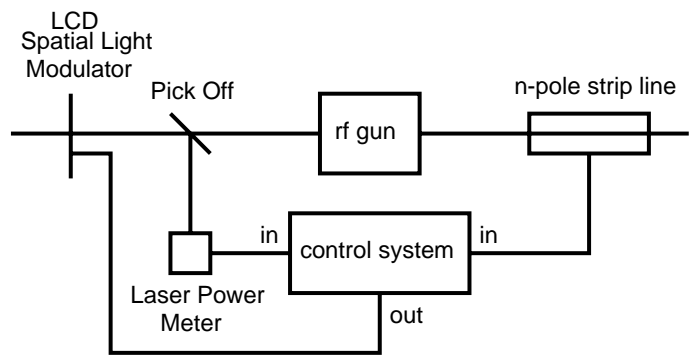

Figure 6.1: A non-invasive feedback system to correct for the 2-D variation of the electron charge distribution.

measurements of the electron beam's transverse charge distribution.

The difference between semiconductor cathodes and metal cathodes is thought to be that the metal cathodes are more robust than semiconductor material with respect to vacuum and handling constraints. We believe this is not the case: metal cathodes should be handled in the same way that semiconductors are handled. Ultra High Vacuum (UHV) transfer system, ion plasma cleaning, and a Low Energy Electron Diffractometer (LEED) should be incorporated into the photocathode handling system of a rf gun. This would mitigate the deleterious effects of exposing the metal photocathode to air and allow for the characterization of the photocathode material in situ. When a UHV transfer system is implemented, the cathode joint might need to revert back to a smaller diameter and the multipacting problem would have to be reexamined. The PWT rf gun could play a major role in this area since its rf field gradient, for optimal emittance compensation, is a factor of 2 smaller than that required by the 1.6 cell $\mathrm{rf}$ gun. In the meantime, an $\mathrm{N}_{2}$ glove box transfer system would allow for the cleaning, transfer, and installation of the 1.6 cell rf gun's cathode plate without exposing it to air.

\section{Thermal Emittance, $\epsilon_{\mathrm{o}}$}

An upper limit of the electron beam's thermal emittance has been experimentally measured as $\epsilon_{\mathrm{o}}=0.80 \pi \mathrm{mm}$ mrad. The conventional wisdom is that this term cannot be eliminated. An interesting means of eliminating the thermal emittance contribution is to design the rf field level at the cathode, the photocathode material, and the 
laser system in such a way as to take advantage of the Schottky effect. By this we mean that the system should be designed using a cathode material with a higher zero field work function, $\phi_{0}$, than that of the laser photon energy. With an applied accelerating field that has been chosen to optimize the emittance compensation process, the effective work function, $\phi_{\text {eff }}$, of the material would be tuned to be less than or equal to the laser photon energy. In this way the excess electron kinetic energy can be tuned by the applied accelerating field such that the electrons' excess kinetic energy is exactly to zero. This would produce a zero thermal emittance electron beam. An alternate method would be to use a tunable laser system, such as Ti:Sapphire, so that the electric field at the cathode can be set by beam dynamics requirements. It should be remembered that the emittance due to the actual physical temperature of the cathode, $\epsilon_{\mathrm{T}}$, will still be an effect that these schemes cannot eliminate. Both of these methods have the drawback that the maximum laser energy will have to be increased, since we are not depending on the Schottky effect to increase the electron yield.

In addition, the use of a spin polarized rf gun operating in the emittance compensation regime would allow for the production of an emittance compensated electron beam with a zero thermal emittance contribution. Since the production of spin polarized electrons necessitates that the photon energy be tuned to the band gap energy of GaAs, there will be no excess electron energy that could manifest itself into a thermal emittance term.

Our theoretical calculations of the thermal emittance contribution to the total emittance budget, with an applied field gradient of $123 \frac{\mathrm{MV}}{\mathrm{m}}$, is equal to $\epsilon_{\mathrm{o}}=0.62 \pi$ $\mathrm{mm}$ mrad. Experimental investigation of the electron velocity distribution at zero field from single crystal copper $\mathrm{Cu}(100)$ is underway by the photocathode materials group at INFN LASE Milano [61]. This work will allow for the calculation of the thermal emittance of $\mathrm{Cu}(100)$ with zero applied field. This is the first step in understanding the thermal emittance of electrons photoemitted from a metal cathode under the influence of an applied accelerating field. 


\subsubsection{Advanced Beam Diagnostics}

The tuning algorithm for all emittance studies presented in this dissertation was to tune the beam line to produce the smallest symmetric beam profile at the output of the second linac section. Subsequent PARMELA [3] simulations indicate that this was not the correct tuning algorithm. Fig. 5.18 represents these PARMELA [3] simulations for a total charge of $0.333 \mathrm{nC}$. This plot indicates that the beam line should be tuned to produce a slightly divergent beam at high energy.

We did not have the ability to tune for minimum transverse emittance. Further work must include the development of an emittance tuning algorithm that allows the beam physicist to tune for minimum transverse emittance and not minimum spot size. A diagnostics station with a uniform array pepper pot and transition radiation screen would make this possible on a single shot basis.

Measurements of the electron beam's slice emittance need to be conducted using a non-perturbing phase space diagnostics. The energy chirp technique used at the ATF should be replaced with a transition radiation screen and a streak camera. This would eliminate the requirement to produce an energy position correlation that perturbs the 6-D phase space of the electron beam in the electron bunch as is presently required at the ATF.

Using the pepper-pot, a transition radiation screen, and a streak camera, at the output of the second linac section would allow for single shot emittance tuning of the electron beam. Also the electron bunch length would be measurable at this location instead of downstream in the dispersive region of the energy spectrometer. 


\section{Appendix A}

\section{Index of Symbols}

\begin{tabular}{ll} 
Symbol & Description \\
\hline $\mathrm{a}$ & Width of the waveguide \\
$\mathrm{a}$ & Maximum spot size \\
$\mathrm{a}_{\mathrm{ij}}$ & ij $^{\text {th }}$ SASE fitting parameter \\
$\mathrm{A}$ & Amperes \\
$\mathrm{A}$ & Vector potential \\
$\mathrm{Al}$ & Aluminum \\
$\mathrm{Au}$ & Gold \\
$\mathrm{AR}$ & Aspect ratio \\
$\mathrm{AWG}$ & American Wire Gauge \\
$\mathrm{A}_{\mathrm{m} 10}$ & TM \\
$\mathrm{A}_{\mathrm{z}}$ & $\mathrm{z}$ component of the vector potential \\
$\mathrm{A}_{\mathrm{eff}}$ & Effective field emitting area \\
AC & Alternating Current \\
ATF & Accelerator Test Facility \\
$\mathrm{b}$ & Height of the waveguide \\
$\mathrm{b}$ & Maximum beam divergence \\
$\mathrm{BPM} 5$ & Beam Profile Screen \\
BPM6 & Beam Profile Screen \\
$\mathrm{B}_{\mathrm{z}}$ & Longitudinal magnetic field \\
&
\end{tabular}




$\begin{array}{ll}\mathrm{B}_{z, \text { max }} & \text { Peak longitudinal magnetic field } \\ \mathrm{B}_{z, \mathrm{BM}} & \text { Longitudinal magnetic field due to Bucking Magnet } \\ \mathrm{B}_{z}(\mathrm{z}=0) & \text { Cathode magnetic field } \\ \mathrm{B}_{z}(\mathrm{r}=0) & \text { On-axis magnetic field } \\ \mathrm{B}_{\mathrm{z}_{\mathrm{o}}} & \text { Cathode magnetic field } \\ \mathrm{B} & \text { Magnetic field vector } \\ \mathrm{B}_{\mathrm{x}} & \text { X-axis magnetic field } \\ \mathrm{BNL} & \text { Brookhaven National Laboratory } \\ \mathrm{c} & \text { Speed of light } \\ \mathrm{cm} & \text { Centi meters } \\ \mathrm{cov}\left(\mathrm{x}_{1}, \mathrm{x}_{2}\right) & \text { Covariance function } \\ \mathrm{C} & \text { Coulomb } \\ \mathrm{CCD} & \text { Charged Coupled Detector } \\ \mathrm{CW} & \text { Continuous Wave } \\ \mathrm{CsTe} & \text { Cesium Telluride } \\ \mathrm{Cu} & \text { Copper } \\ \mathrm{C}_{1} & \text { Half cell capacitance } \\ \mathrm{C}_{2} & \text { Full cell capacitance } \\ \mathrm{C}_{\mathrm{i}} & \text { Cosine like beam transport function } \\ \mathrm{d} & \text { Coupling aperture thickness } \\ \mathrm{d} & \text { Half cell length perturbation } \\ \mathrm{db} & \text { Decibel } \\ \mathrm{dl} & \text { Infinitesimal integration vector } \\ \mathrm{dv} & \text { Infinitesimal volume cavity } \\ \mathrm{d} \tau & \text { Infinitesimal volume perturbing object } \\ \mathrm{DC} & \text { Direct Current } \\ \mathrm{DESY} & \text { Deutsches Elektronen-Synchrotron } \\ \mathrm{e} & \text { Charge of an electron } \\ \mathrm{e}_{\mathrm{o}} & \sqrt{1-\frac{1_{2}^{2}}{1_{1}^{2}}} \\ \mathrm{E} & \text { Energy } \\ \mathrm{E} & \text { Electric field vector } \\ & \end{array}$




\begin{tabular}{|c|c|}
\hline $\mathrm{E}_{\mathrm{i}}$ & $\mathrm{i}^{\text {th }}$ cell Electric field vector \\
\hline ECOMP & POISSON input deck \\
\hline EDM & Electro Discharge Machining \\
\hline $\mathrm{E}_{\text {beam }}$ & Average beam energy \\
\hline $\mathrm{E}_{\mathrm{o}}$ & Peak accelerating gradient \\
\hline $\mathrm{E}_{\mathrm{r}}$ & Radial electric field \\
\hline $\mathrm{E}_{\mathrm{z}}$ & Accelerating field gradient \\
\hline $\mathrm{E}_{z, \max }$ & Accelerating field gradient maximum \\
\hline $\mathrm{E}_{\mathrm{z}, \pi}$ & $\pi$-mode accelerating field gradient \\
\hline $\mathrm{E}_{\mathrm{z}, 0}$ & 0-mode accelerating field gradient \\
\hline $\mathrm{E}_{\mathrm{i}}$ & $\mathrm{i}^{\text {th }}$ cell electric field strength \\
\hline $\mathrm{E}_{1}$ & Half cell field gradient \\
\hline $\mathrm{E}_{2}$ & Full cell field gradient \\
\hline $\mathrm{E}_{2} / \mathrm{E}_{1}$ & Field balance \\
\hline $\mathbf{E}_{\alpha}$ & Electric field at perturbing object \\
\hline $\mathrm{E}_{\gamma}$ & Laser photon energy \\
\hline $\mathrm{E}\left(\mathrm{e}_{\mathrm{o}}\right)$ & Elliptic integrals of the second kind \\
\hline $\mathrm{f}$ & Generic function \\
\hline$f_{o}$ & 0 -mode frequency \\
\hline $\mathrm{f}_{\pi}$ & $\pi$-mode frequency \\
\hline $\mathrm{f}_{1}$ & Independent half cell frequency \\
\hline $\mathrm{f}_{2}$ & Independent full cell frequency \\
\hline fsec & Femto seconds \\
\hline $\mathrm{f}\left(\epsilon_{\mathrm{f}}\right)$ & Fermi-Dirac distribution \\
\hline FB & Field Balance \\
\hline $\mathrm{F}_{\mathrm{r}}$ & Radial force \\
\hline FEL & Free Electron Laser \\
\hline FWHM & Full Width Half Maximum \\
\hline FWTM & Full Width Tenth Maximum \\
\hline $\mathrm{FWHM}_{\mathrm{z}}$ & Longitudinal flat-top \\
\hline$C$ & Gauss \\
\hline
\end{tabular}




\begin{tabular}{|c|c|}
\hline GaAs & Gallium Arsenide \\
\hline $\mathrm{h}$ & Planks constant \\
\hline $\mathrm{h}_{1}$ & Resistive coupling slot $\mathrm{z}$ dimension \\
\hline $\mathrm{h}_{2}$ & Capacitive coupling slot $\mathrm{z}$ dimension \\
\hline $\mathbf{H}$ & Magnetic field vector \\
\hline $\mathrm{Hz}$ & Hertz \\
\hline $\mathrm{HP}$ & High Pressure \\
\hline HPOP1 & Beam profile screen \\
\hline $\mathrm{H}_{2}$ & Diatomic Hydrogen \\
\hline $\mathrm{H}_{1}$ & Magnetic field strength \\
\hline $\mathrm{H}_{\phi}$ & Azimuthal magnetic field \\
\hline $\mathbf{H}_{\alpha}$ & Magnetic field at perturbing object \\
\hline $\mathrm{H}\left(\mathrm{q}_{\mathrm{k}}, \mathrm{p}_{\mathrm{k}}, \mathrm{t}\right)$ & Hamiltonian \\
\hline input_file & PARMELA input file name \\
\hline $\mathrm{i}$ & $\sqrt{-1}$ \\
\hline I & Current \\
\hline ID & Inner Diameter \\
\hline $\mathrm{I}_{\mathrm{p}}$ & Peak current \\
\hline $\mathrm{I}_{\mathrm{A}}$ & Alfvén current \\
\hline $\mathrm{I}_{1}$ & Half cell current \\
\hline $\mathrm{I}_{2}$ & Full cell current \\
\hline $\mathrm{IE}$ & Invariant Envelope \\
\hline INFN & Istituto Nazionale di Fisica Nucleare \\
\hline $\mathrm{J}$ & Joule \\
\hline $\mathbf{J}$ & Current density \\
\hline $\mathrm{k}$ & Wave number \\
\hline $\mathrm{k}_{\mathrm{o}}$ & Wave number \\
\hline $\mathrm{kG}$ & Kilo Gauss \\
\hline $\mathrm{k}_{\mathrm{B}}$ & Boltzmann's constant \\
\hline $\mathrm{K}$ & Perturbing object constant \\
\hline KV & Kapchinskij-Vladimirskij distribution \\
\hline
\end{tabular}




\begin{tabular}{|c|c|}
\hline $\mathrm{KE}_{\mathrm{o}}$ & Initial kinetic energy \\
\hline $\mathrm{K}\left(\mathrm{e}_{\mathrm{o}}\right)$ & Elliptic integrals of the first kind \\
\hline $\ln$ & Log base e \\
\hline$l_{1}$ & Half major axis of rf coupling slot \\
\hline$l_{2}$ & Half minor axis of $\mathrm{rf}$ coupling slot \\
\hline $\mathrm{L}$ & Beam-lets radial length \\
\hline $\mathrm{L}$ & Distance between screens \\
\hline $\mathrm{L}$ & Distance between BPM5 and BPM6 \\
\hline $\mathrm{L}_{\mathrm{o}}$ & Drift distance \\
\hline $\lim _{Q_{t} \rightarrow 0}$ & Limit as total charge goes to zero \\
\hline $\mathrm{L}_{\mathrm{eff}}$ & Effective magnetic length \\
\hline $\mathrm{L}_{\mathrm{g}}$ & Power gain length \\
\hline $\mathrm{L}_{\mathrm{r}}$ & Rayleigh range \\
\hline $\mathrm{L}_{\mathrm{w}}$ & Wiggler period \\
\hline $\mathrm{L}_{1 \mathrm{~d}}$ & 1-D gain length \\
\hline $\mathrm{L}_{1}$ & Half cell inductance \\
\hline $\mathrm{L}_{2}$ & Full cell inductance \\
\hline LAEEE & Laser Assisted Explosive Electron Emission \\
\hline \multirow[t]{2}{*}{ LASE } & Laboratorio Acceleratori e \\
\hline & Superconduttivita' Applicata \\
\hline $\mathrm{LC}$ & Inductor Capacitor model \\
\hline LCD & Liquid Crystal Display \\
\hline LCLS & Linear Coherent Light Source \\
\hline LEED & Low Energy Electron Diffractometer \\
\hline $\mathrm{m}$ & Meters \\
\hline $\mathrm{meV}$ & milli electron Volts \\
\hline $\mathrm{mW}$ & milli Watts \\
\hline mill & 0.001 inches \\
\hline $\mathrm{mm}$ & millimeters \\
\hline msec & milli seconds \\
\hline$m_{0}$ & Electron rest mass \\
\hline
\end{tabular}




\begin{tabular}{|c|c|}
\hline $\operatorname{mrad}$ & milli radians \\
\hline $\mathrm{M}$ & Mutual inductance \\
\hline $\mathrm{MeV}$ & Million electron Volts \\
\hline $\mathrm{Mg}$ & Magnesium \\
\hline $\mathrm{MHz}$ & Mega Hertz \\
\hline MV & Mega Volts \\
\hline MW & Mega Watts \\
\hline $\mathrm{n}$ & Total number of macro particles \\
\hline & used in PARMELA simulations \\
\hline $\mathrm{nC}$ & nano Coulomb \\
\hline $\mathrm{nm}$ & nano meters \\
\hline nsec & nano seconds \\
\hline $\mathrm{Nd}: \mathrm{YAG}$ & Neodymium:YAG \\
\hline NEA & Negative Electron Affinity \\
\hline NGP & Next Generation Photoinjector \\
\hline $\mathrm{NLC}$ & Next Linear Collider \\
\hline $\mathrm{N}_{\mathrm{u}}$ & Number of undulator periods \\
\hline $\mathrm{N}_{\mathrm{e}^{-}}$ & Number of macro-particles \\
\hline $\mathrm{N}_{2}$ & Diatomic nitrogen \\
\hline outgraf & PARGRAF output file name \\
\hline outpar & PARMELA output file name \\
\hline OD & Outer Diameter \\
\hline OFHC & Oxygen Free High Conductive \\
\hline $\mathrm{pC}$ & pico Coulomb \\
\hline psec & pico seconds \\
\hline $\mathrm{p}_{\mathrm{i}}$ & Hamiltonian momentum \\
\hline $\mathrm{p}_{\mathrm{x}}$ & $\mathrm{x}$ component of momentum \\
\hline $\mathrm{p}_{\mathrm{x}, \mathrm{rms}}$ & $\mathrm{x}$ component of rms momentum \\
\hline $\mathrm{p}_{\mathrm{z}}$ & z component of momentum \\
\hline$\dot{\mathrm{p}}_{\mathrm{i}}$ & Hamiltonian time derivative of momentum \\
\hline $\mathrm{P}$ & SASE output Power \\
\hline
\end{tabular}




\begin{tabular}{|c|c|}
\hline $\mathrm{PRF}$ & Pulse Repetition Frequency \\
\hline $\mathrm{P}_{\text {beam }}$ & Beam power \\
\hline $\mathrm{P}_{\mathrm{o}}$ & Power loss \\
\hline $\mathrm{P}_{\mathrm{n}}$ & Noise power \\
\hline $\mathrm{P}_{\text {sat }}$ & Saturation power \\
\hline PAC97 & 1997 Particle Accelerator Conference \\
\hline PWT & Plane Wave Transformer \\
\hline $\mathrm{q}_{\mathrm{i}}$ & Hamiltonian position \\
\hline$\dot{\mathrm{q}}_{\mathrm{i}}$ & Hamiltonian time derivative of position \\
\hline QE & Quantum Efficiency \\
\hline $\mathrm{QE}(\mathrm{x}, \mathrm{y})$ & $2-D$ variation of quantum efficiency \\
\hline Q & Quality factor \\
\hline$Q_{\text {ext }}$ & External Q \\
\hline$Q_{0}$ & Unloaded Q \\
\hline $\mathrm{Q}_{\mathrm{L}}$ & Loaded Q \\
\hline $\mathrm{Q}_{\mathrm{t}}$ & Total charge \\
\hline $\mathrm{r}$ & Radius of the electron beam \\
\hline $\mathrm{rad}$ & Radians \\
\hline $\mathrm{rf}$ & radio-frequency \\
\hline $\mathrm{rms}$ & root mean squared \\
\hline $\mathrm{r}_{\mathrm{o}}$ & Cathode laser spot size \\
\hline $\mathrm{R}$ & Radius \\
\hline $\mathrm{R}_{\mathrm{IE}}$ & Beam radius scaled with invariant envelope \\
\hline $\mathrm{R}_{\mathrm{L}}$ & Load impedance \\
\hline $\mathrm{R}_{\max }$ & Maximum PARMELA electron source size \\
\hline $\mathrm{R}_{\mathrm{o}}$ & Laser spot size \\
\hline $\mathrm{R}_{\mathrm{rms}}$ & HPOP1 Spot size \\
\hline $\mathrm{R}_{\mathrm{s}, \pi}$ & Shunt impedance $\pi$-mode \\
\hline $\mathrm{R}_{\mathrm{s}, 0}$ & Shunt impedance 0 -mode \\
\hline sec & Seconds \\
\hline Sapphire & $\mathrm{Al}_{2} \mathrm{O}_{3}$ \\
\hline
\end{tabular}




\begin{tabular}{|c|c|}
\hline $\mathrm{SST}$ & Stainless Steel \\
\hline STPH & Standard Temperature, Pressure, and Humidity \\
\hline $\mathrm{S}_{\mathrm{ij}}$ & ij element of the scattering matrix \\
\hline $\mathrm{S}_{\mathrm{i}}$ & Sine like beam transport function \\
\hline $\mathrm{S}_{1}$ & Screen one \\
\hline $\mathrm{S}_{2}$ & Screen two \\
\hline SASE & Self-Amplified Spontaneous Emission \\
\hline SLAC & Stanford Linear Accelerator Center \\
\hline SSRL & Stanford Synchrotron Radiation Laboratory \\
\hline $\mathrm{t}$ & Time \\
\hline $\mathrm{T}$ & Temperature in Kelvin \\
\hline Ti : Sapphire & Titanium:Sapphire \\
\hline $\mathrm{TE}$ & Transverse Electric \\
\hline TEM & Transverse Electro Magnetic \\
\hline $\mathrm{TM}$ & Transverse Magnetic \\
\hline TRL & Transmission Reflection Load \\
\hline $\mathrm{T}_{1}$ & Resistive coupling slot thickness \\
\hline $\mathrm{T}_{2}$ & Capacitive coupling slot thickness \\
\hline $\mathrm{TM}_{010}$ & Transverse Magnetic 010 mode \\
\hline TTF & TESLA Test Facility \\
\hline $\mathrm{u}$ & Total stored energy \\
\hline UHV & Ultra High Vacuum \\
\hline uv & ultraviolet \\
\hline UCLA & University of California Los Angles \\
\hline $\mathrm{v}$ & Velocity of electron \\
\hline $\mathbf{v}$ & Vector velocity of electron \\
\hline $\mathrm{v}_{\mathrm{x}}$ & $\mathrm{x}$ component of the electron velocity \\
\hline $\mathrm{v}_{\mathrm{rms}}$ & $\mathrm{x}$ component of the electron rms velocity \\
\hline VSWR & Voltage standing wave ratio \\
\hline $\mathrm{V}$ & Volts \\
\hline $\mathrm{V}_{\max }$ & Voltage maximum \\
\hline
\end{tabular}




\begin{tabular}{|c|c|}
\hline $\mathrm{V}_{\min }$ & Voltage minimum \\
\hline $\mathrm{V}_{\mathrm{i}}$ & $\mathrm{i}^{\text {th }}$ cell voltage \\
\hline $\mathrm{V}_{1}$ & Half cell voltage \\
\hline $\mathrm{V}_{2}$ & Full cell voltage \\
\hline$\dot{\mathrm{V}}_{\mathrm{i}}$ & $\mathrm{i}^{\text {th }}$ cell voltage time derivative \\
\hline$\dot{\mathrm{V}}_{1}$ & Half cell voltage time derivative \\
\hline$\dot{\mathrm{V}}_{2}$ & Full cell voltage time derivative \\
\hline $\mathrm{V}_{2}^{\rho}$ & Volume of 2-D phase space \\
\hline $\mathrm{V}_{\mathrm{j}}^{+}$ & Incident voltage at port $\mathrm{j}$ \\
\hline $\mathrm{V}_{\mathrm{i}}^{-}$ & Reflected voltage at port i \\
\hline $\mathrm{w}_{1}$ & Resistive coupling slot $\mathrm{x}$ dimension \\
\hline $\mathrm{w}_{2}$ & Capacitive coupling slot $\mathrm{x}$ dimensior \\
\hline W & Watts \\
\hline $\mathrm{x}$ & $\mathrm{X}$-dimension \\
\hline $\mathrm{x}_{\mathrm{rms}}^{\prime}$ & $\mathrm{X}$-dimension rms divergence \\
\hline $\mathrm{y}$ & Y-dimension \\
\hline $\mathrm{y}_{\mathrm{o}}$ & Total integration length \\
\hline $\mathrm{Y}$ & Admittance \\
\hline$Y_{0}$ & Normalized admittance \\
\hline $\mathrm{z}$ & Position in the electron bunch \\
\hline $\mathrm{z}$ & Variable beam line position \\
\hline $\mathrm{z}_{\mathrm{O}}$ & Fixed beam line position \\
\hline $\mathrm{Z}_{\mathrm{o}}$ & Impedance of free space, $377 \Omega$ \\
\hline$\alpha$ & Normalized field strength parameter \\
\hline$\alpha_{c}$ & FEL coupling coefficient \\
\hline$\alpha_{\mathrm{o}}$ & $\mathrm{k}_{\mathrm{o}}\left[\left(\frac{\lambda_{\mathrm{rf}}}{\lambda_{c}}\right)^{2}-1\right]$ \\
\hline$\alpha_{\mathrm{T}}$ & Twiss parameter \\
\hline$\beta$ & Normalized velocity \\
\hline$\beta$ & Field enhancement factor \\
\hline$\beta_{\theta}$ & Azimuthal normalized velocity \\
\hline
\end{tabular}




\begin{tabular}{|c|c|}
\hline$\beta_{\mathrm{z}}$ & Z normalized velocity \\
\hline$\beta_{\mathrm{T}}$ & Twiss parameter \\
\hline$\beta_{\mathrm{rf}}$ & rf coupling constant \\
\hline$\beta_{\gamma}$ & Field enhancement factor of \\
\hline & the photoemitting surface \\
\hline$\gamma$ & Normalized energy \\
\hline$\gamma_{\mathrm{T}}$ & Twiss parameter \\
\hline$\gamma^{\prime}$ & Accelerating gradient \\
\hline 10 & $\mathrm{k}_{\mathrm{o}}\left(1-\left[\frac{\lambda_{\mathrm{rf}}}{2 \mathrm{a}}\right)\right]$ \\
\hline$\delta \phi$ & Phase difference between the two linac sections \\
\hline$\delta \theta$ & Relative angular rotation \\
\hline$\delta \mathrm{v}$ & Integration over the cavity volume \\
\hline$\delta \mathrm{x}_{\mathrm{rms}}$ & Change in rms spot size \\
\hline$\delta \tau$ & Integration over perturbing object volume \\
\hline$\epsilon$ & Permittivity of free space \\
\hline$\epsilon$ & Transverse emittance \\
\hline$\epsilon_{r}$ & FEL beam emittance \\
\hline$\epsilon_{\mathrm{n}, \mathrm{rms}}$ & Transverse normalized rms emittance \\
\hline$\epsilon_{\mathrm{g}, \mathrm{rms}}$ & Geometric rms emittance \\
\hline$\epsilon_{\mathrm{SC}}$ & Space charge emittance \\
\hline$\epsilon_{\mathrm{rf}}$ & Radio-frequency emittance \\
\hline$\epsilon_{\mathrm{mp}}$ & Multi-pole field emittance \\
\hline$\epsilon_{\mathrm{B}_{\mathrm{z}}}$ & Magnetic field emittance \\
\hline$\epsilon_{\mathrm{O}}$ & Thermal emittance \\
\hline$\epsilon_{\mathrm{T}}$ & Physical temperature emittance \\
\hline$\epsilon_{\mathrm{f}}$ & Fermi energy \\
\hline$\epsilon_{\mathrm{n}, \mathrm{rms}}^{\mathrm{x}, \mathrm{y}}$ & Transverse normalized rms emittance (x or y) \\
\hline$\epsilon_{\mathrm{n}, \mathrm{rms}}^{\text {slice }}$ & Transverse normalized rms slice emittance \\
\hline$\epsilon_{\mathrm{n}, \mathrm{rms}}^{\text {projected }}$ & Transverse normalized rms projected emittance \\
\hline$\eta$ & Universal scaling parameter \\
\hline$\eta_{d}$ & Gain length reduction due to diffraction \\
\hline
\end{tabular}




\begin{tabular}{|c|c|}
\hline$\eta_{\epsilon}$ & Gain length reduction due to emittance \\
\hline$\eta_{\gamma}$ & Gain length reduction due to energy spread \\
\hline$\theta$ & Azimuthal coordinate \\
\hline$\theta_{\mathrm{o}}$ & Laser injection phase \\
\hline$\theta_{\mathrm{o}}$ & Angular integration limit \\
\hline$\theta_{\mathrm{r}}$ & Angular divergence \\
\hline$\dot{\theta}$ & Time derivative of angular rotation \\
\hline$\Theta$ & Azimuthal coordinate \\
\hline$\Theta_{0}$ & PARMELA injection phase \\
\hline$\lambda$ & Wavelength \\
\hline$\lambda$ & FEL wavelength \\
\hline$\lambda$ & Laser wavelength $\lambda=266 \mathrm{~nm}$ \\
\hline$\lambda_{\mathrm{rf}}$ & rf wavelength \\
\hline$\lambda_{\mathrm{c}}$ & Cutoff wavelength \\
\hline$\lambda_{\mathrm{g}}$ & Guide wavelength \\
\hline$\lambda_{\mathrm{r}}$ & Undulator radiation wavelength \\
\hline$\lambda_{\mathrm{u}}$ & Undulator period \\
\hline$\mu$ & Permeability of free space \\
\hline$\mu J$ & micro Joule \\
\hline$\mu \mathrm{sec}$ & micro seconds \\
\hline$\mu_{\mathrm{cp}}$ & Chemical potential \\
\hline$\mu_{\mathrm{i}}$ & Universal space charge factor \\
\hline$\mu \mathrm{m}$ & micro meters (microns) \\
\hline$\pi$ & Ratio of the circumference \\
\hline & to the diameter of a circle \\
\hline$\rho$ & Phase space density \\
\hline$\rho(\mathrm{x}, \mathrm{y})$ & Electron bunch charge distribution \\
\hline$\rho_{\mathrm{IE}}$ & Space charge vs emittance regime parameter \\
\hline$\rho_{\mathrm{P}}$ & Pierce parameter \\
\hline$\omega$ & Perturbed angular frequency \\
\hline$\omega_{0}$ & Unperturbed angular frequency \\
\hline
\end{tabular}




\begin{tabular}{|c|c|}
\hline$\Omega_{\gamma}$ & Laser polarization angle \\
\hline$\Omega$ & Ohms \\
\hline$\Omega^{2}$ & Normalized focusing frequency \\
\hline$\sigma$ & Sigma matrix \\
\hline$\sigma_{\mathrm{e}}$ & rms energy spread \\
\hline$\sigma_{\mathrm{r}}$ & Effective source radius \\
\hline$\sigma_{\mathrm{r}}$ & PARMELA rms electron source size \\
\hline$\sigma_{\mathrm{u}}$ & Correlated error \\
\hline$\sigma_{\mathrm{z}}$ & Electron bunch length (psec) \\
\hline$\sigma_{\mathrm{x}}$ & Transverse rms beam size $(\mathrm{mm})$ \\
\hline$\sigma_{\mathrm{y}}$ & Transverse rms beam size (mm) \\
\hline$\sigma_{\mathrm{x}, \mathrm{y}}$ & Transverse rms beam size (mm) \\
\hline$\sigma_{\mathrm{z}}$ & Longitudinal Gaussian \\
\hline & rms beam size (psec) \\
\hline$\sigma_{\mathrm{g}, \mathrm{rms}}^{\mathrm{x}}$ & rms spot size \\
\hline$\sigma_{\mathrm{g}, \mathrm{rms}}^{\mathrm{x}^{\prime}}$ & rms beam divergence \\
\hline$\sigma_{\mathrm{i}, 11}$ & $\mathrm{i}^{\text {th }} \mathrm{rms}$ beam spot size squared \\
\hline$\sigma_{1}$ & rms beam size BPM5 \\
\hline$\sigma_{2}$ & rms beam size BPM6 \\
\hline$\phi$ & Laser injection phase \\
\hline$\phi_{\mathrm{o}}$ & Zero field work function of $\mathrm{Cu}$ \\
\hline$\phi_{\text {eff }}$ & Effective work function of $\mathrm{Cu}$ \\
\hline$\Phi$ & Magnetic flux \\
\hline$\Phi_{11 \mathrm{rf}}$ & low level rf phase \\
\hline$\Phi_{\mathrm{o}}$ & Initial magnetic flux \\
\hline$\tau$ & Electron bunch length \\
\hline$\tau_{95 \%}$ & Electron bunch length $\left(4 \sigma_{\mathrm{z}}\right)$ \\
\hline$\nabla$ & Del operator \\
\hline$\partial$ & Dirac delta function \\
\hline $\mathrm{J}_{0}$ & Zeroth order Bessel function \\
\hline$\frac{d}{d t}$ & Total time derivative \\
\hline
\end{tabular}




$\begin{array}{ll}\frac{\partial}{\partial \mathrm{x}} & \text { Partial derivative with respect to } \mathrm{x} \\ \frac{\mathrm{d} \rho}{\mathrm{dt}} & \text { Total time derivative of the phase space density } \\ \# \mathrm{~N}_{\mathrm{e}} & \text { Number of electron } \\ \# \mathrm{~N}_{\gamma} & \text { Number of photons } \\ \frac{\delta \gamma}{\gamma} & \text { Energy spread } \\ \left\langle\mathrm{g}^{2}\right\rangle & \text { RMS average of the transverse } \\ \AA & \text { Space charge field form factor } \\ 10^{\circ} & \text { Angstrom } \\ { }^{\circ} \mathrm{C} & 10 \text { degrees } \\ { }^{\prime} & \text { degrees Celsius } \\ \mathcal{F} & \text { inches } \\ \left\langle\mathrm{x}^{2}\right\rangle & \text { Universal scaling function } \\ \left\langle\mathrm{x}^{\prime 2}\right\rangle & \text { Mean square beam size } \\ \left\langle\mathrm{xx}^{\prime}\right\rangle & \text { Mean square beam divergence }\end{array}$




\section{Bibliography}

[1] Herman Winick. The linac coherent light source (LCLS): a fourth-generation light source using the SLAC linac. Journal of Electron Spectroscopy and Related Phenomena, 75:1-8, 1995.

[2] B. E. Carlsten. NEW PHOTOELECTRIC INJECTOR DESIGN FOR THE LOS ALAMOS NATIONAL LABORATORY XUV FEL ACCELERATOR. Nuclear Instruments and Methods in Physics Research, A285:313-319, 1989.

[3] L. M. Young. Personal Communication.

[4] Luca Serafini and James B. Rosenzweig. Envelope analysis of intense relativistic quasilaminar beams in rf photoinjectors: A theory of emittance compensation. Phys. Rev. E, 55(6):7565-7590, 1997.

[5] K. Halbach and R. F. Holsinger. SUPERFISH-A COMPUTER PROGRAM FOR EVALUATION OF RF CAVITIES WITH CYLINDRICAL SYMMETRY. Particle Accelerators, 7:213-222, 1976.

[6] R. Bonifacio, C. Pellegrini and L. M. Narducci. COLleCtive InSTABILITIES AND HIGH-GAIN REGIME IN A FREE ELECTRON LASER. Optics Communications, 50(6):373-378, 1984.

[7] J. B. Murphy and C. Pellegrini. Generation of high-intensity coherent radiation in the soft-x-ray and vacuum-ultraviolet region. J. Opt. Soc. Am. B, 2(1):259264, 1985. 
[8] The LCLS Design Study Group, M. Cornacchia, Design Study Group Leader. LCLS Design Study Report. Technical Report SLAC-R-521, Stanford Linear Accelerator Center, April 1998.

[9] M. Xie. DESIGN OPTIMIZATION FOR AN X-RAY FREE ELECTRON LASER DRIVEN BY THE SLAC LINAC. In Gennari [62], pages 183-185.

[10] J. L. Adamski et al. THE BOEING DOUBLE SUBHARMONIC ELECTRON INJECTOR - PERFORMANCE MEASUREMENTS. IEEE Transactions on Nuclear Science, NS-32(5):2994-2996, 1985.

[11] R. Alley et al. The Stanford linear accelerator polarized electron source. Nuclear Instruments and Methods in Physics Research, A365:1-27, 1995.

[12] J. Rosenzweig and E. Colby. Charge and Wavelength Scaling of RF Photoinjector Designs. TESLA 95-04, 1995.

[13] Michael Borland. A HIGH-BRIGHTNESS THERMIONIC MICROWAVE ELECTRON GUN. PhD thesis, Stanford University, 1991.

[14] Pamela Kung et al. Generation and Measurement of 50-fs (rms) Electron Pulses. Physical Review Letters, 73(7):967-970, 1994.

[15] R. L. Sheffield et al. THE LOS ALAMOS PHOTOINJECTOR PROGRAM. Nuclear Instruments and Methods in Physics Research, A272:222-226, 1988.

[16] K. Batchelor, J. Sheehan and M. Woodle. Design and Modeling of a $5 \mathrm{MeV}$ Radio Frequency Electron Gun. BNL-41766, 1988.

[17] D. T. Palmer et al. MICROWAVE MEASUREMENTS OF THE BNL/SLAC/UCLA 1.6 CELL PHOTOCATHODE RF GUN. In Gennari [62], pages 982-984.

[18] D. T. Palmer et al. SIMUlations OF THE BNL/SLAC/UCLA 1.6 CELL EMITTANCE COMPENSATED RF GUN LOW ENERGY BEAM LINE. In Gennari [62], pages 2432-2434. 
[19] Eric Ralph Colby. Design, Construction and Testing of a Radiofrequency Photoinjector for the Next Generation Linear Collider. PhD thesis, University of California Los Angles, 1997.

[20] A. D. Yeremian et al. A PROPOSED INJECTOR FOR THE LCLS LINAC. In Gennari [63].

[21] J. D. Lawson. The Physics of Charged-Particle Beams. Oxford University Press, 1988.

[22] L. D. Landau and E. M. Lifshitz. Mechanics. Pergamon, Tarrytown, New York, 1994.

[23] A. Septier, editor. Applied Charged Particle Optics. Academic Press, 1980.

[24] Kwang-Je Kim. RF AND SPACE-CHARGE EFFECTS IN LASER-DRIVEN RF ELECTRON GUNS. Nuclear Instruments and Methods in Physics Research, A275:210-218, 1989.

[25] R. B. Neal, editor. THE STANFORD TWO-MILE ACCELERATOR. W. A. Benjamin, Inc., New York, New York, 1968.

[26] W. K. H. Panofsky and W. A. Wenzel. Some Considerations Concerning the Transverse Deflection of Charged Particles in Radio-Frequency Fields. THE REVIEW OF SCIENTIFIC INSTRUMENTS, 27:967, 1956.

[27] I. M. Kapchinskij and V.V. Vladimirskij. LIMITATIONS OF PROTON BEAM CURRENT IN A STRONG FOCUSING LINEAR ACCELERATOR ASSOCIATED WITH BEAM SPACE CHARGE. In L. Kowarski, editor, Proceedings of the International Conference on High Energy Accelerators, Geneva, 1959. CERN.

[28] H. Busch. Berechnung der Bahn von Kathodenstrahlen in axialsymmetrischen elektromagnetisichen Felde. Annalen der Physik, 81:974-993, 1926.

[29] Walter Schottky. Über den Austritt von Elektronen aus Glühdrähten bei verzögernden Potentialen. Annalen der Physik, 44:1011-1032, 1914. 
[30] Jim Clendenin and G.A. Mullhollan. High Quantum Yield, Low Emittance Electron Sources. SLAC-PUB-7r60, 1998.

[31] Neil W. Ashcroft and N. David Mermin. Solid State Physics. Saunders College, 1976.

[32] Luca Serafini. Personal Communication.

[33] L. Serafini. IMPROVING THE BEAM QUALITY OF RF GUNS BY CORRECTION OF RF AND SPACE CHARGE EFFECTS. In Wurtele [64].

[34] David M. Pozar. Microwave Engineering. Addison-Wesley, Reading, Massachusetts, 1993.

[35] Edward L. Ginzton. Microwave Measurements. McGraw-Hill, New York, New York, 1957.

[36] J. C. Slater. Microwave Electronics. Reviews of Modern Physics, 18(4):441-512, 1946.

[37] K. Narula. Chemical Cleaning of Oxygen Free Electronic Grade (O.F.E.) Copper. Technical Report Process Specification C01, Stanford Linear Accelerator Center, 1998.

[38] XiJie Wang et al. Design and Construction a Full Copper Photocathode RF Gun. In Steven T. Corneliussen, editor, Proceedings of the 1993 Particle Accelerator Conference, volume 4, pages 3000-3002, Piscataway, New Jersey, 1993. IEEE.

[39] R. Klatt et al. MAFIA - A Three-Dimensional Electromagnetic CAD System for Magnets, RF Structures, and Transient Wake-Field Calculations. In I. E. Campisi, editor, 1986 LINEAR ACCELERATOR CONFERENCE PROCEEDINGS, pages 276-278, Stanford CA, 1986. Stanford Linear Accelerator Center.

[40] A. M. Vetter and R. P. Friedman. MEASUREMENT OF FIELD ASYMMETRY IN ACCELERATING CAVITIES WITH WAVEGUIDE COUPLERS. In J. Leemann, editor, 1988 LINEAR ACCELERATOR CONFERENCE PROCEEDINGS, volume CEBAF-Report-89-001, pages 58-60. CEBAF, 1989. 
[41] Greg Loew. Personal Communication.

[42] H. A. Bethe. Theory of Diffraction by Small Holes. The Physical Review, 66(7 and 8):163-182, 1944.

[43] J. Gao. Analytical formula for the coupling coefficient $\beta$ of a cavity-waveguide coupling system. Nuclear Instruments and Methods in Physics Research, A309:510, 1991.

[44] X. J. Wang et al. Experimental characterization of the high-brightness electron photoinjector. Nuclear Instruments and Methods in Physics Research, A375:82$86,1996$.

[45] Klaus Halbach. DESIGN CONSIDERATIONS FOR A LUMPED SOLENOID. Technical Report LBL 4270, LAWRENCE BERKELEY LABORATORY, 1975.

[46] J. D. Jackson. Classical Electrodynamics. John Wiley and Sons, Second edition, 1975.

[47] Ilan Ben-Zvi. The BNL ACCELERATOR TEST FACILITY AND EXPERIMENTAL PROGRAM. In Wurtele [64], pages 590-607.

[48] R. Malone et al. THE BNL ACCELERATOR TEST FACILITY CONTROL SYSTEM. In D. Axen and R. Poutissou, editors, Conference Record of the Eighth Conference on Real-time Computer Application in Nuclear, Particle and Plasma Physics, volume TRI-93-1, pages 289-291, Vancouver, B.C., June 1993. TRIUMF.

[49] Helmut Wiedemann. Particle Accelerator Physics. Springer-Verlag, 1993.

[50] T. Srinivasan-Rao et al. Performance of Magnesium Cathode in the S-Band rf gun. In Gennari [63].

[51] XiJie Wang. High-brightness Electron Accelerator Injection System and Experimental Studies of Laser-induced Explosive Electron Emission. PhD thesis, University of California Los Angles, 1992. 
[52] J. W. Wang. RF PROPERTIES OF PERIODIC ACCELERATING STRUCTURES FOR LINEAR COLLIDERS. Technical Report SLAC-Report-339, Stanford Linear Accelerator Center, Stanford CA, 1989.

[53] X. Qiu, K. Batchelor, I. Ben-Zvi and X-J. Wang. Demonstration of Emittance Compensation thought the Measurement of the Slice Emittance of a 10-ps Electron Bunch. Physical Review Letters, 76(20):3723-3726, 1996.

[54] X.J. Wang, X. Qiu and I. Ben-Zvi. Experimental observation of high-brightness microbunching in a photocathode rf electron gun. Physical Review E, 54(4A):3121-3124, 1996.

[55] Donald W. Feldman el at. Experimental Results from the Los Alamos FEL Photoinjector. IEEE J. Quantum Electronic, 27(12):2636-2643, 1991.

[56] D. T. Palmer et al. Experimental Results of a Single Emittance Compensation Solenoidal Magnet. In Gennari [63].

[57] X. J. Wang et al. Experimental Results of the ATF in-line Injection System. In Gennari [62].

[58] D. T. Palmer et al. Emittance Studies of the BNL/SLAC/UCLA 1.6 cell Photocathode rf Gun. In Gennari [63].

[59] D. T. Palmer, J. B. Rosenzweig and L. Serafini. Space Charge Code Checking Group. This group was formed at 1997 Particle Accelerator Conference to insure the reliability of space charged dominated particle tracking codes.

[60] D. T. Palmer. Photocathode Guns for Single Pass X-ray FELs. In Rodolfo Bonifacio and William A. Barletta, editors, TOWARDS X-RAY FREE ELECTRON LASERS, 413, pages 155-168, Woodbury, New York, 1997. American Institute of Physics.

[61] P. Michelato. Personal Communication. 
[62] Laurie Gennari, editor. Proceedings of the 1995 Particle Accelerator Conference and International Conference Conference on High-Energy Accelerators, New Jersy, 1995. IEEE.

[63] Laurie Gennari, editor. Proceedings of the 1997 Particle Accelerator Conference and International Conference Conference on High-Energy Accelerators, New Jersy, 1997. IEEE.

[64] Jonathan S. Wurtele, editor. AIP CONFERENCE PROCEEDINGS, volume 279. AIP PRESS, 1992. 